\title{
Molecular Packing and Symmetry of Two-Dimensional
}

\section{Crystals}

\author{
Katherine E. Plass, Adam L. Grzesiak, and Adam J. Matzger ${ }^{*}$ \\ Department of Chemistry and the Macromolecular Science and Engineering Program, University of \\ Michigan, Ann Arbor, Michigan 48109-1055
}

\section{Supporting Information}

1) Details of database construction and structural assignment

2) Expanded discussion of selection criteria

3) Comparison of the symmetry of the position on which a molecule sits in the unit cell and the highest possible molecular symmetry

4) Three-dimensional space group frequencies

5) List of all papers included in the 2DSD

6) The structural assignments for high and medium resolution entries into the 2DSD 
1) Details of database construction and structural assignment

To obtain candidates for inclusion in the database, a thorough literature search was conducted using Scifinder Scholar (Version 2004, Copyright (C2003 American Chemical Society) with the keyword combinations in Table S1. Candidates selected from the abstracts returned were examined in detail to determine that they met selection criteria. It was attempted to include all such monolayers, and crosschecks between different keyword combinations indicated that most papers were found more than once. No abstracts or dissertations were included, and a few papers could not be obtained through library sources. Non-review articles published before the end of 2004 (315 total) were included.

Table S1. Keyword combinations used in Scifinder Scholar to find papers for the 2DSD.

\begin{tabular}{|l|l|l|}
\hline Keyword 1 & Keyword 2 & Number of hits from 1988-2004 \\
\hline 2D & graphite & 608 \\
\hline 2D & scanning tunneling & 446 \\
\hline 2D & STM & 394 \\
\hline graphite & interface & 7219 \\
\hline graphite & monolayer & 1864 \\
\hline graphite & scanning tunneling & 2217 \\
\hline interface & scanning tunneling & 2984 \\
\hline interface & STM & 2395 \\
\hline monolayer & scanning tunneling & 3170 \\
\hline self-assembly & graphite & 520 \\
\hline STM & graphite & 1819 \\
\hline STM & monolayer & 2667 \\
\hline two-dimensional & graphite & 1312 \\
\hline two-dimensional & scanning tunneling & 1113 \\
\hline two-dimensional & STM & 759 \\
\hline
\end{tabular}

Papers were analyzed and the data were collected into a Chemfinder (ChemOffice Ultra 2004, Copyright (C2004 Cambridgesoft) database. Each distinct monolayer within a paper was given an entry in the database, so every compound, combination of compounds, and different packing motif for the same compound was included. This resulted in multiple entries for some compounds that were reported more than once, in a process analogous to that of the Cambridge Structural Database. Repetitions were 
included in the database, as resolution often improved in later papers, but only the highest resolution entry for each form was considered in the analyses described in the paper.

A structural description of the overlayer that matched the STM image was developed. The plane group and the number of molecules in the asymmetric unit were determined $\left(Z^{\prime}\right)$. The crystal class (whether the unit cell was oblique, rectangular, square, or hexagonal) and number of molecules in the unit cell (Z) followed from this assignment. A resolution descriptor, which is roughly comparable to the R-factor of a crystal structure, was included to describe the certainty of the plane group, crystal class, $Z$, and $Z^{\prime}$ values. The resolution descriptors were broadly grouped into high, medium, low, and disordered. Monolayers in the high and medium categories were sufficiently resolved that a confident symmetry assignment could be made. These monolayers were included in the analyses in the paper. A common example of a medium resolution monolayer was that of the $n$-alkanes where the presence of glide planes was not always apparent from the image; however their structure was confirmed by the highest resolution images and by neutron diffraction studies. Low resolution monolayer images were such that if an assignment could be made, little confidence was placed in it, or no assignment was realistic. Disordered monolayers were such that periodicity was often randomly broken. Some of these monolayers could be assigned local symmetry; for instance monolayers that randomly change the orientation of columns can be described by the dominant symmetry relationship. Such monolayers, however, have been excluded in the present analysis.

Although STM provides the highest resolution of any method of examining surface structure, not every atom is generally apparent. The highest apparent symmetries were assigned, assuming no disorder without experimental evidence. Thus, in the cases where the monolayers appeared more symmetric than the molecules themselves, the plane group symmetry assignments were limited to those consistent with molecular symmetry. In cases where unresolved portions of the molecule might break symmetry, it was not assumed that they did.

The site symmetry, or the symmetry position of the molecule in the unit cell, was assigned for every molecule. If a molecule resided on a two-fold rotation, the site symmetry is 2 , whereas a molecule in a 
general position is denoted by 1 . This convention was continued for the 3-, 4- and 6-fold rotations, while $\mathrm{m}$ represents occupation of a mirror plane, $\mathrm{g}$ represents occupation of a glide plane, and $2 \mathrm{~mm}$ simultaneous occupation of a two-fold rotation and perpendicular mirror planes. The highest possible molecular symmetry was identified by inspection. Thus, monolayers that consist of symmetry inequivalent molecules or multiple components required several assignments of site and molecular symmetry. For single component monolayers composed of symmetry inequivalent molecules, multiple site symmetries were assigned. For multicomponent entries, molecular symmetry and site symmetry were assigned for each component. The order in which the site symmetry is listed is the same as for the molecular symmetry. 
The method of physisorbed monolayer formation could impact the observed trends in monolayer structure in several ways. Firstly, the method of deposition influences which molecules are included. The 2DSD is largely made up of molecules decorated with alkyl chains, which aid in assembly and solubilization. There is an overall lack of highly symmetric molecules and this certainly affects the overall plane group frequencies. For example, using other formation methods, four-fold symmetric molecules have been assembled into monolayers with p4 symmetry, ${ }^{1}$ which was not observed in the analysis of the 2DSD presented here. This effect has been accounted for in the discussion of tendency toward close packing. Measurement of the plane group frequencies for molecules sitting on a general position controls for the influence of the various molecular symmetries included in the dataset. An additional factor in rapid monolayer preparation procedures is the increased possibility of observing higher-energy, non-equilibrium structures. While this was the basis for exclusion of these structures, there exists some evidence suggesting that the results obtained in this study will be relevant to these other systems. While extensive comparisons have not been made, there are reports of molecules forming the same packing motif when assemblies are created using more than one method, ${ }^{2,3,4}$ although this is not always the case. ${ }^{5}$ Furthermore, some examples do exist of vacuum-deposited structures on graphite, including a report detailing the importance of packing efficiency on motif and growth mode selection, ${ }^{1,6}$ suggesting that the tendency toward close packing will play an important role in dictating the assembly of these structures.

(1) Lei, S. B.; Wang, C.; Yin, S. X.; Wang, H. N.; Xi, F.; Liu, H. W.; Xu, B.; Wan, L. J.; Bai, C. L. J. Phys. Chem. B 2001, 105, 10838-10841.

(2) Shao, X.; Luo, X. C.; Hu, X. Q.; Wu, K. J. Phys. Chem. B 2006, 110, 1288-1293.

(3) Qiu, X. H.; Wang, C.; Zeng, Q. D.; Xu, B.; Yin, S. X.; Wang, H. N.; Xu, S. D.; Bai, C. L. J. Am. Chem. Soc. 2000, 122, 5550-5556.

(4) Trimesic acid adopts two different packing motifs at the graphite interface, both of which have been observed in solution (Lackinger, M.; Griessl, S.; Heckl, W. A.; Hietschold, M.; Flynn, G. 
W., Langmuir, 2005, 21, 4984-4988) and in ultra-high vacuum (Griess1, S.; Lackinger, M.; Edelwirth, M.; Hietschold, M.; Heckl, W. M., Single Mol., 2002, 3, 25-31)

(5) Widmer, I.; Hubler, U.; Stöhr, M.; Merz, L.; Güntherodt, H. J.; Hermann, B. A.; Samorí, P.; Rabe, J. P.; Rheiner, P. B.; Greiveldinger, G.; Murer, P. Helv. Chim. Acta 2002, 85, 4255-4263.

(6) Fujiwara, E.; Isoda, S.; Ogawa, T.; Kobayashi, T.; Yamashita, Y. Surf. Sci. 2001, 487, 118-126. 
3) Comparison of the symmetry of the position on which a molecule sits in the unit cell and the highest possible molecular symmetry

Table S2. Below is a comparison of the symmetry of the position on which a molecule sits in the unit cell compared to the highest possible molecular symmetry. This reveals how often molecular symmetry is lost when forming a two-dimensional periodic structure.

\begin{tabular}{|l|l|}
\hline $\begin{array}{l}\text { molecular } \\
\text { symmetry }\end{array}$ & $\begin{array}{l}\text { number of } \\
\text { occurrences }\end{array}$ \\
\hline general position \\
\hline $\mathrm{C}(\mathrm{s})$ & 195 \\
\hline $\mathrm{C}(1)$ & 63 \\
\hline $\mathrm{C}(2 \mathrm{v})$ & 36 \\
\hline $\mathrm{C}(2 \mathrm{~h})$ & 3 \\
\hline $\mathrm{S}(6)$ & 2 \\
\hline $\mathrm{D}(3 \mathrm{~h})$ & 2 \\
\hline $\mathrm{C}(6 \mathrm{~h})$ & 2 \\
\hline $\mathrm{C}(3)$ & 2 \\
\hline $\mathrm{D}(3 \mathrm{~d})$ & 1 \\
\hline $\mathrm{D}(2 \mathrm{~h})$ & 1 \\
\hline $\mathrm{C}(2)$ & 1 \\
\hline two-fold rotation \\
\hline $\mathrm{C}(2 \mathrm{~h})$ & 53 \\
\hline $\mathrm{D}(2 \mathrm{~h})$ & 14 \\
\hline $\mathrm{C}(2 \mathrm{v})$ & 13 \\
\hline $\mathrm{C}(2 \mathrm{~h})$ & 9 \\
\hline $\mathrm{C}(2)$ & 4 \\
\hline $\mathrm{C}(6 \mathrm{~h})$ & 3 \\
\hline $\mathrm{D}(6 \mathrm{~h})$ & 2 \\
\hline $\mathrm{D}(4 \mathrm{~h})$ & 2 \\
\hline $\mathrm{C}(4)$ & 2 \\
\hline $\mathrm{D}(3 \mathrm{~d})$ & 1 \\
\hline $\mathrm{D}(24 \mathrm{~h})$ & 1 \\
\hline & \\
\hline
\end{tabular}

\begin{tabular}{|l|l|}
\hline $\begin{array}{l}\text { molecular } \\
\text { symmetry }\end{array}$ & $\begin{array}{l}\text { number of } \\
\text { occurrences }\end{array}$ \\
\hline $\begin{array}{l}\text { simultaneous two-fold } \\
\text { rotation and perpendicular } \\
\text { mirror planes }\end{array}$ \\
\hline $\mathrm{D}(4 \mathrm{~h})$ & 1 \\
\hline $\mathrm{D}(2 \mathrm{~h})$ & 1 \\
\hline three-fold rotation \\
\hline $\mathrm{D}(3 \mathrm{~h})$ & 9 \\
\hline $\mathrm{C}(3 \mathrm{~h})$ & 1 \\
\hline $\mathrm{C}(3)$ & 1 \\
\hline six-fold rotation \\
\hline $\mathrm{D}(6 \mathrm{~h})$ & 1 \\
\hline $\mathrm{D}(6 \mathrm{~d})$ & 1 \\
\hline $\mathrm{C}(6 \mathrm{~h})$ & 1 \\
\hline $\mathrm{C}(6)$ & 1 \\
\hline mirror plane & \\
\hline $\mathrm{C}(2 \mathrm{v})$ & 15 \\
\hline $\mathrm{C}(2 \mathrm{~h})$ & 3 \\
\hline $\mathrm{C}(\mathrm{s})$ & 2 \\
\hline $\mathrm{D}(3 \mathrm{~h})$ & 1 \\
\hline
\end{tabular}


4) Three-dimensional space group frequencies

The frequencies of occurrence of the space groups in the CSD were determined using the November 2004 version, version 5.26. To eliminate repeated entries, only refcodes not containing numbers were included. Both organic and organometallic entries were considered. While the exact percentages varied slightly depending on the $\mathrm{R}$ factors of the structures and dataset, only the space groups $P 2_{1} / c, P \overline{1}$, $P 22_{1} 2_{1}, C 2 / c, P 2_{1}$, and $P b c a$ were observed in more than $2.0 \%$ of the entries and are in agreement with previous in-depth space group frequency analyses (Brock, C. P.; Dunitz, J. D. Chem. Mater., 1994, 6, 1118-1127). Pbca was not mentioned in Kitaigorodskii, A. I., Organic Chemical Crystallography, 1959, (Consultants Bureau, New York) as a close-packing group, but has since been included (Wilson, A.J.C., Acta Cryst., 1993, A49, 210-212).

Table S3. Space group frequencies from the CSD.

\begin{tabular}{|c|c|c|c|}
\hline $\begin{array}{l}\text { space group } \\
\text { number }\end{array}$ & $\begin{array}{c}\text { space group } \\
\text { name }\end{array}$ & $\begin{array}{c}\text { total number of } \\
\text { observations in initial } \\
\text { structure } \\
\text { determinations }\end{array}$ & $\begin{array}{c}\text { percentage of } \\
\text { observations }\end{array}$ \\
\hline 14 & $P 2_{1} / c$ & 101679 & 35.60 \\
\hline 2 & $P \overline{\overline{1}}$ & 64259 & 22.50 \\
\hline 19 & $P 2_{1} 2_{1} 2_{1}$ & 23753 & 8.32 \\
\hline 15 & $C 2 / c$ & 22182 & 7.77 \\
\hline 4 & $P 2_{1}$ & 15922 & 5.57 \\
\hline 61 & $P b c a$ & 10295 & 3.60 \\
\hline 33 & $P_{n a 2_{1}}$ & 4141 & 1.45 \\
\hline 62 & Pnma & 3701 & 1.30 \\
\hline 9 & $C c$ & 3073 & 1.08 \\
\hline 1 & $P 1$ & 2761 & 0.97 \\
\hline 60 & $P b c n$ & 2512 & 0.88 \\
\hline 5 & $C 2$ & 2448 & 0.86 \\
\hline 29 & $\mathrm{Pca2}_{1}$ & 2042 & 0.71 \\
\hline 11 & $P 2_{1} / m$ & 1651 & 0.58 \\
\hline 148 & $R \overline{3}$ & 1610 & 0.56 \\
\hline 13 & $P 2 / c$ & 1580 & 0.55 \\
\hline 12 & $C 2 / m$ & 1400 & 0.49 \\
\hline 18 & $P 2{ }_{1} 2_{1} 2$ & 1245 & 0.44 \\
\hline
\end{tabular}




\begin{tabular}{|c|c|c|c|}
\hline 7 & $P c$ & 1089 & 0.38 \\
\hline 56 & $P c c n$ & 960 & 0.34 \\
\hline 43 & $F d d 2$ & 915 & 0.32 \\
\hline 88 & $I 4_{1} / a$ & 906 & 0.32 \\
\hline 92 & $P 4_{1} 2_{1} 2$ & 638 & 0.22 \\
\hline 20 & $C 22_{1}$ & 498 & 0.17 \\
\hline 36 & $C m c 2_{1}$ & 464 & 0.16 \\
\hline 96 & $P 4_{3} 2_{1} 2$ & 412 & 0.14 \\
\hline 82 & $I \overline{4}$ & 399 & 0.14 \\
\hline 64 & $C m c a$ & 383 & 0.13 \\
\hline 146 & $R 3$ & 377 & 0.13 \\
\hline 114 & $P \overline{4} 2_{1} c$ & 376 & 0.13 \\
\hline 86 & $P 4_{2} / n$ & 373 & 0.13 \\
\hline 167 & $R-3 c$ & 337 & 0.12 \\
\hline 57 & $P b c m$ & 323 & 0.11 \\
\hline 63 & $C m c m$ & 304 & 0.11 \\
\hline $\begin{array}{c}\text { total number of } \\
\text { observations }\end{array}$ & & 285623 & all others $\leq$ \\
\hline
\end{tabular}


5) List of all papers included in the 2DSD

(1) Plass, K. E.; Kim, K.; Matzger, A. J. J. Am. Chem. Soc. 2004, 126, 9042-9053.

(2) Mu, Z.; Yang, X.; Wang, Z.; Zhang, X.; Zhao, J.; Bo, Z. Langmuir 2004, 20, 8892-8896.

(3) Mamdouh, W.; Uji-i, H.; Gesquière, A.; De Feyter, S.; Amabilino, D. B.; Abdel-Mottaleb, M. M. S.; Veciana, J.; De Schryver, F. C. Langmuir 2004, 20, 9628-9635.

(4) Lensen, M. C.; van Dingenen, S. J. T.; Elemans, J. A. A. W.; Dijkstra, H. P.; van Klink, G. P. M.; van Koten, G.; Gerritsen, J. W.; Speller, S.; Nolte, R. J. M.; Rowan, A. E. Chem. Commun. 2004, 762-763.

(5) Nishio, S.; Yoshidome, M.; Uji-i, H.; Hobley, J.; Fukumura, H.; Zachariasse, K. A. Chem. Lett. 2004, 33, 1506-1507.

(6) Mu, Z.-C.; Kong, J.-F.; Wang, Y.; Ye, L.; Yang, G.-D.; Zhang, X. ChemPhysChem 2004, 5, 202-208.

(7) De Feyter, S.; Abdel-Mottaleb, M. M. S.; Schuurmans, N.; Verkuij1, B. J. V.; Van Esch, J. H.; Feringa, B. L.; De Schryver, F. C. Chem.-Eur. J. 2004, 10, 1124-1132.

(8) Schuurmans, N.; Uji-i, H.; Mamdouh, W.; De Schryver, F. C.; Feringa, B. L.; Van Esch, J.; De Feyter, S. J. Am. Chem. Soc. 2004, 126, 13884-13885.

(9) Mu, Z.; Wang, Z.; Zhang, X.; Ye, K.; Wang, Y. J. Phys. Chem. B 2004, 108, 19955-19959.

(10) Kalsani, V.; Ammon, H.; Jäckel, F.; Rabe, J. P.; Schmittel, M. Chem.-Eur. J. 2004, 10, 54815492.

(11) Jonkheijm, P.; Miura, A.; Zdanowska, M.; Hoeben, F. J. M.; De Feyter, S.; Schenning, A. P. H. J.; De Schryver, F. C.; Meijer, E. W. Angew. Chem., Int. Edit. 2004, 43, 74-78.

(12) Gesquière, A.; Jonkheijm, P.; Hoeben, F. J. M.; Schenning, A. P. H. J.; De Feyter, S.; De Schryver, F. C.; Meijer, E. W. Nano Lett. 2004, 4, 1175-1179.

(13) Mamdouh, W.; Uji-i, H.; Dulcey, A. E.; Percec, V.; De Feyter, S.; De Schryver, F. C. Langmuir 2004, 20, 7678-7685.

(14) Ikeda, T.; Asakawa, M.; Goto, M.; Miyake, K.; Ishida, T.; Shimizu, T. Langmuir 2004, 20, 5454-5459.

(15) Jäckel, F.; Yin, X.; Samorí, P.; Tchebotareva, N.; Watson, M. D.; Venturini, A.; Müllen, K.; Rabe, J. P. Synth. Met. 2004, 147, 5-9.

(16) Lackinger, M.; Griessl, S.; Markert, T.; Jamitzky, F.; Heck1, W. M. J. Phys. Chem. B 2004, 108, 13652-13655.

(17) Mourran, A.; Beginn, U.; Zipp, G.; Möller, M. Langmuir 2004, 20, 673-679.

(18) Perronet, K.; Charra, F. Surf. Sci. 2004, 551, 213-218. 
(19) Samorì, P.; Yin, X.; Tchebotareva, N.; Wang, Z.; Pakula, T.; Jäckel, F.; Watson, M. D.; Venturini, A.; Müllen, K.; Rabe, J. P. J. Am. Chem. Soc. 2004, 126, 3567-3575.

(20) Wei, Y.; Kannappan, K.; Flynn, G. W.; Zimmt, M. B. J. Am. Chem. Soc. 2004, 126, 5318-5322.

(21) Watson, M. D.; Jäckel, F.; Severin, N.; Rabe, J. P.; Müllen, K. J. Am. Chem. Soc. 2004, 126, 1402-1407.

(22) Griessl, S. J. H.; Lackinger, M.; Jamitzky, F.; Markert, T.; Hietschold, M.; Heckl, W. M. Langmuir 2004, 20, 9403-9407.

(23) Gomar-Nadal, E.; Abdel-Mottaleb, M. M. S.; De Feyter, S.; Veciana, J.; Rovira, C.; Amabilino, D. B.; De Schryver, F. C. Chem. Commun. 2003, 906-907.

(24) Olson, J. A.; Bühlmann, P. Anal. Chem. 2003, 75, 1089-1093.

(25) Wouters, D.; Höppener, S.; Lunkwitz, R.; Chi, L. F.; Fuchs, H.; Schubert, U. S. Adv. Funct. Mater. 2003, 13, 277-280.

(26) Takajo, D.; Nemoto, T.; Kurata, H.; Isoda, S.; Ozaki, H.; Mazaki, Y. Thin Solid Films 2003, 438, 428-432.

(27) Uji-i, H.; Yoshidome, M.; Hobley, J.; Hatanaka, K.; Fukumura, H. Phys. Chem. Chem. Phys. 2003, 5, 4231-4235.

(28) Abdel-Mottaleb, M. M. S.; Gomar-Nadal, E.; De Feyter, S.; Zdanowska, M.; Veciana, J.; Rovira, C.; Amabilino, D. B.; De Schryver, F. C. Nano Lett. 2003, 3, 1375-1378.

(29) Yasuda, S.; Shigekawa, H. Jpn. J. Appl. Phys., Part 1 2003, 42, 4901-4904.

(30) Qiu, D.; Ye, K.; Wang, Y.; Zou, B.; Zhang, X.; Lei, S.; Wan, L. Langmuir 2003, 19, 678-681.

(31) Lei, S.-B.; Wang, C.; Fan, X.-L.; Wan, L.-J.; Bai, C.-L. Langmuir 2003, 19, 9759-9763.

(32) Abdel-Mottaleb, M. M. S.; De Feyter, S.; Sieffert, M.; Klapper, M.; Müllen, K.; De Schryver, F. C. Langmuir 2003, 19, 8256-8261.

(33) Tchebotareva, N.; Yin, X.; Watson, M. D.; Samorí, P.; Rabe, J. P.; Müllen, K. J. Am. Chem. Soc. 2003, 125, 9734-9739.

(34) Miura, A.; Chen, Z.; Uji-i, H.; De Feyter, S.; Zdanowska, M.; Jonkheijm, P.; Schenning, A. P. H. J.; Meijer, E. W.; Würthner, F.; De Schryver, F. C. J. Am. Chem. Soc. 2003, 125, 1496814969.

(35) Giorgi, T.; Lena, S.; Mariani, P.; Cremonini, M. A.; Masiero, S.; Pieraccini, S.; Rabe, J. P.; Samorí, P.; Spada, G. P.; Gottarelli, G. J. Am. Chem. Soc. 2003, 125, 14741-14749.

(36) Wintgens, D.; Yablon, D. G.; Flynn, G. W. J. Phys. Chem. B 2003, 107, 173-179.

(37) Gesquière, A.; Jonkheijm, P.; Schenning, A.; Mena-Osteritz, E.; Bäuerle, P.; De Feyter, S.; De Schryver, F. C.; Meijer, E. W. J. Mater. Chem. 2003, 13, 2164-2167. 
(38) De Feyter, S.; Larsson, M.; Schuurmans, N.; Verkuijl, B.; Zoriniants, G.; Gesquière, A.; AbdelMottaleb, M. M.; van Esch, J.; Feringa, B. L.; van Stam, J.; De Schryver, F. Chem.-Eur. J. 2003, 9, 1198-1206.

(39) Samorí, P.; Francke, V.; Enkelmann, V.; Müllen, K.; Rabe, J. P. Chem. Mater. 2003, 15, $1032-$ 1039.

(40) Nishino, T.; Ito, T.; Umezawa, Y. J. Electroanal. Chem. 2003, 550-551, 125-129.

(41) Nanjo, H.; Qian, P.; Yokoyama, T.; Suzuki, T. M. Jpn. J. Appl. Phys., Part 1 2003, 42, 65606563.

(42) Matzger, A. J.; Kim, K. Polym. Mater. Sci. Eng. 2003, 89, 825-826.

(43) Su, C.; Yu, S. H.; Shu, C. R.; Wang, H. H.; Lai, C. K. Synth. Met. 2003, 137, 915-916.

(44) De Feyter, S.; Gesquière, A.; Klapper, M.; Müllen, K.; De Schryver, F. C. Nano Lett. 2003, 3, 1485-1488.

(45) Elemans, J. A. A. W.; Lensen, M. C.; Gerritsen, J. W.; van Kempen, H.; Speller, S.; Nolte, R. J. M.; Rowan, A. E. Adv. Mater. 2003, 15, 2070-2073.

(46) Binnemans, K.; Sleven, J.; De Feyter, S.; De Schryver, F. C.; Donnio, B.; Guillon, D. Chem. Mater. 2003, 15, 3930-3938.

(47) Sakata, I.; Miyamura, K. Chem. Commun. 2003, 156-157.

(48) Takajo, D.; Fujiwara, E.; Irie, S.; Nemoto, T.; Isoda, S.; Ozaki, H.; Toda, N.; Tomii, S.; Magara, T.; Mazaki, Y.; Yamamoto, G. J. Cryst. Growth 2002, 237-239, 2071-2075.

(49) De Feyter, S.; Gesquière, A.; De Schryver, F. C.; Keller, U.; Müllen, K. Chem. Mater. 2002, 14, 989-997.

(50) Abdel-Mottaleb, M. M. S.; Schuurmans, N.; De Feyter, S.; Van Esch, J.; Feringa, B. L.; De Schryver, F. C. Chem. Commun. 2002, 1894-1895.

(51) Ziener, U.; Lehn, J.-M.; Mourran, A.; Möller, M. Chem.-Eur. J. 2002, 8, 951-957.

(52) Samorí, P.; Simpson, C. D.; Müllen, M.; Rabe, J. P. Langmuir 2002, 18, 4183-4185.

(53) Nemoto, T.; Adachi, Y.; Fujiwara, E.; Isoda, S. Mol. Cryst. Liq. Cryst. Sci. Technol., Sect. A 2002, 389, 97-104.

(54) Widmer, I.; Hubler, U.; Stöhr, M.; Merz, L.; Güntherodt, H. J.; Hermann, B. A.; Samorí, P.; Rabe, J. P.; Rheiner, P. B.; Greiveldinger, G.; Murer, P. Helv. Chim. Acta 2002, 85, 4255-4263.

(55) Su, C.; Shu, C.-R.; Wu, C.-C. Liq. Cryst. 2002, 29, 1169-1176.

(56) Yablon, D. G.; Wintgens, D.; Flynn, G. W. J. Phys. Chem. B 2002, 106, 5470-5475.

(57) Feng, C. L.; Zhang, Y. J.; Jin, J.; Song, Y. L.; Xie, L. Y.; Qu, G. R.; Jiang, L.; Zhu, D. B. Surf. Sci. 2002, 513, 111-118. 
(58) Hoeppener, S.; Chi, L. F.; Fuchs, H. Nano Lett. 2002, 2, 459-463.

(59) Samorí, P.; Severin, N.; Simpson, C. D.; Müllen, K.; Rabe, J. P. J. Am. Chem. Soc. 2002, 124, 9454-9457.

(60) Jin, J.; Li, Y.; Zhang, Y.; Yang, W.; Chen, X.; Song, Y.; Zhao, Y.; Bai, Y.; Li, T.; Jiang, L. Colloids Surf., A 2002, 198-200, 249-253.

(61) Kim, K.; Matzger, A. J. J. Am. Chem. Soc. 2002, 124, 8772-8773.

(62) Mena-Osteritz, E. Adv. Mater. 2002, 14, 609-616.

(63) Taki, S.; Kai, S. Jpn. J. Appl. Phys., Part 1 2001, 40, 4187-4192.

(64) Nishino, T.; Bühlmann, P.; Ito, T.; Umezawa, Y. Surf. Sci. 2001, 490, L579-L584.

(65) Samorí, P.; Engelkamp, H.; de Witte, P.; Rowan, A. E.; Nolte, R. J. M.; Rabe, J. P. Angew. Chem., Int. Edit. 2001, 40, 2348-2350.

(66) De Feyter, S.; Gesquière, A.; Wurst, K.; Amabilino, D. B.; Veciana, J.; De Schryver, F. C. Angew. Chem., Int. Edit. 2001, 40, 3217-3220.

(67) Samorí, P.; Fechtenkötter, A.; Jäckel, F.; Böhme, T.; Müllen, K.; Rabe, J. P. J. Am. Chem. Soc. 2001, 123, 11462-11467.

(68) Mena-Osteritz, E.; Bäuerle, P. Adv. Mater. 2001, 13, 243-246.

(69) Ohshiro, T.; Ito, T.; Bühlmann, P.; Umezawa, Y. Anal. Chem. 2001, 73, 878-883.

(70) Samorí, P.; Jäckel, F.; Ünsal, Ö.; Godt, A.; Rabe, J. P. ChemPhysChem 2001, 2, 461-464.

(71) Hoeppener, S.; Chi, L. F.; Wonnemann, J.; Erker, G.; Fuchs, H. Surf. Sci. 2001, 487, 9-14.

(72) Kaneda, Y.; Stawasz, M. E.; Sampson, D. L.; Parkinson, B. A. Langmuir 2001, 17, 6185-6195.

(73) Abdel-Mottaleb, M. M. S.; De Feyter, S.; Gesquière, A.; Sieffert, M.; Klapper, M.; Müllen, K.; De Schryver, F. C. Nano Lett. 2001, 1, 353-359.

(74) Höger, S.; Bonrad, K.; Mourran, A.; Beginn, U.; Möller, M. J. Am. Chem. Soc. 2001, 123, 56515659 .

(75) Isoda, S.; Nemoto, T.; Fujiwara, E.; Adachi, Y.; Kobayashi, T. J. Cryst. Growth 2001, 229, 574579.

(76) Prokhorova, S. A.; Sheiko, S. S.; Mourran, A.; Azumi, R.; Beginn, U.; Zipp, G.; Ahn, C.-H.; Holerca, M. N.; Percec, V.; Möller, M. Langmuir 2000, 16, 6862-6867.

(77) Krömer, J.; Rios-Carreras, I.; Furhmann, G.; Musch, C.; Wunderlin, M.; Debaerdemacker, T.; Mena-Osteritz, E.; Bäuerle, P. Angew. Chem., Int. Edit. 2000, 39, 3481-3486.

(78) Gottarelli, G.; Masiero, S.; Mezzina, E.; Pieraccini, S.; Rabe, J. P.; Samorí, P.; Spada, G. P. Chem.-Eur. J. 2000, 6, 3242-3248. 
(79) De Feyter, S.; Gesquière, A.; De Schryver, F.; Meiners, C.; Sieffert, M.; Müllen, K. Langmuir 2000, 16, 9887-9894.

(80) Gesquière, A.; Abdel-Mottaleb, M. M. S.; De Feyter, S.; De Schryver, F. C.; Schoonbeek, F.; van Esch, J.; Kellogg, R. M.; Feringa, B. L.; Calderone, A.; Lazzaroni, R.; Brédas, J. L. Langmuir 2000, 16, 10385-10391.

(81) Lim, R.; Li, J.; Li, S. F. Y.; Feng, Z.; Valiyaveettil, S. Langmuir 2000, 16, 7023-7030.

(82) Ito, S.; Wehmeier, M.; Brand, J. D.; Kübel, C.; Epsch, R.; Rabe, J. P.; Müllen, K. Chem.-Eur. J. 2000, 6, 4327-4342.

(83) Stawasz, M. E.; Sampson, D. L.; Parkinson, B. A. Langmuir 2000, 16, 2326-2342.

(84) Yablon, D. G.; Giancarlo, L. C.; Flynn, G. W. J. Phys. Chem. B 2000, 104, 7627-7635.

(85) Qian, P.; Nanjo, H.; Sanada, N.; Yokoyama, T.; Suzuki, T. M. Chem. Lett. 2000, 1118-1119.

(86) Azumi, R.; Götz, G.; Debaerdemaeker, T.; Bäuerle, P. Chem.-Eur. J. 2000, 6, 735-744.

(87) Qian, P.; Nanjo, H.; Yokoyama, T.; Suzuki, T. M.; Akasaka, K.; Orhui, H. Chem. Commun. 2000, 2021-2022.

(88) Qiu, X. H.; Wang, C.; Yin, S. X.; Zeng, Q. D.; Xu, B.; Bai, C. L. J. Phys. Chem. B 2000, 104, 3570-3574.

(89) Ito, S.; Herwig, P. T.; Böhme, T.; Rabe, J. P.; Rettig, W.; Müllen, K. J. Am. Chem. Soc. 2000, $122,7698-7706$.

(90) Padowitz, D. F.; Messmore, B. W. J. Phys. Chem. B 2000, 104, 9943-9946.

(91) Latterini, L.; Pourtois, G.; Moucheron, C.; Lazzaroni, R.; Brédas, J.-L.; Mesmaeker, A. K.-D.; De Schryver, F. C. Chem.-Eur. J. 2000, 6, 1331-1336.

(92) Yatabe, T.; Harbison, M. A.; Brand, J. D.; Wagner, M.; Müllen, K.; Samorí, P.; Rabe, J. P. J. Mater. Chem. 2000, 10, 1519-1525.

(93) Stecher, R.; Gompf, B.; Münter, J. S. R.; Effenberger, F. Adv. Mater. 1999, 11, 927-931.

(94) Qian, P.; Nanjo, H.; Yokoyama, T.; Suzuki, T. M. Chem. Lett. 1999, 1229-1230.

(95) Vollmer, M. S.; Effenberger, F.; Stecher, R.; Gompf, B.; Eisenmenger, W. Chem.-Eur. J. 1999, 5, 96-101.

(96) Noh, J.; Lee, D.; Hara, M.; Lee, H.; Sasabe, H.; Knoll, W. Jpn. J. Appl. Phys., Part 1 1999, 38, 3897-3900.

(97) Stecher, R.; Drewnick, F.; Gompf, B. Langmuir 1999, 15, 6490-6494.

(98) Fang, H. B.; Giancarlo, L. C.; Flynn, G. W. J. Phys. Chem. B 1999, 103, 5712-5715.

(99) Baker, R. T.; Mougous, J. D.; Brackley, A.; Patrick, D. L. Langmuir 1999, 15, 4884-4891. 
(100) Gesquière, A.; Abdel-Mottaleb, M. M.; De Schryver, F. C.; Sieffert, M.; Müllen, K. Langmuir 1999, 15, 6821-6824.

(101) Kirschbaum, T.; Azumi, R.; Mena-Osteritz, E.; Bäuerle, P. New J. Chem. 1999, 23, 241-250.

(102) Qian, P.; Nanjo, H.; Yokoyama, T.; Suzuki, T. M. Chem. Commun. 1999, 1197-1198.

(103) Hansen, T.; Itoua, S.; Kamounah, F. S.; Christensen, J. B.; Bjørnholm, T.; Schaumburg, K.; Bechgaard, K.; Wilkes, S. B. J. Mater. Chem. 1999, 9, 1107-1113.

(104) Claypool, C. L.; Faglioni, F.; Matzger, A. J.; Goddard, W. A.; Lewis, N. S. J. Phys. Chem. B 1999, 103, 9690-9699.

(105) Claypool, C. L.; Faglioni, F.; Goddard, W. A.; Lewis, N. S. J. Phys. Chem. B 1999, 103, 70777080.

(106) Samorí, P.; Francke, V.; Müllen, K.; Rabe, J. P. Chem.-Eur. J. 1999, 5, 2312-2317.

(107) Kuwahara, Y.; Saito, A.; Aono, M. Seisan to Gijutsu 1999, 51, 55-58.

(108) De Feyter, S.; Grim, P. C. M.; Rücker, M.; Vanoppen, P.; Meiners, C.; Sieffert, M.; Valiyaveettil, S.; Müllen, K.; De Schryver, F. C. Angew. Chem., Int. Edit. 1998, 37, 1223-1226.

(109) Qian, P.; Nanjo, H.; Yokoyama, T.; Suzuki, T. M.; Miyashita, T. Chem. Commun. 1998, 943944.

(110) Qian, P.; Nanjo, H.; Yokoyama, T.; Suzuki, T. M. Chem. Lett. 1998, 1133-1134.

(111) Fang, H. B.; Giancarlo, L. C.; Flynn, G. W. J. Phys. Chem. B 1998, 102, 7311-7315.

(112) Fang, H. B.; Giancarlo, L. C.; Flynn, G. W. J. Phys. Chem. B 1998, 102, 7421-7424.

(113) Giancarlo, L. C.; Fang, H. B.; Rubin, S. M.; Bront, A. A.; Flynn, G. W. J. Phys. Chem. B 1998, 102, 10255-10263.

(114) Giancarlo, L.; Cyr, D.; Muyskens, K.; Flynn, G. W. Langmuir 1998, 14, 1465-1471.

(115) Charra, F.; Cousty, J. Phys. Rev. Lett. 1998, 80, 1682-1685.

(116) Charra, F.; Cousty, J. Opt. Mater. 1998, 9, 386-389.

(117) Hibino, M.; Sumi, A.; Tsuchiya, H.; Hatta, I. J. Phys. Chem. B 1998, 102, 4544-4547.

(118) Lee, H. S.; Iyengar, S.; Musselman, I. H. Langmuir 1998, 14, 7475-7483.

(119) Schulze, J.; Stevens, F.; Beebe, T. P. J. Phys. Chem. B 1998, 102, 5298-5302.

(120) Iyer, V. S.; Yoshimura, K.; Enkelmann, V.; Epsch, R.; Rabe, J. P.; Müllen, K. Angew. Chem., Int. Edit. 1998, 37, 2696-2699.

(121) Claypool, C. L.; Faglioni, F.; Goddard, W. A.; Gray, H. B.; Lewis, N. S.; Marcus, R. A. J. Phys. Chem. B 1997, 101, 5978-5995. 
(122) Grim, P. C. M.; Vanoppen, P.; Rücker, M.; De Feyter, S.; Valiyaveettil, S.; Moessner, G.; Müllen, K.; De Schryver, F. C. J. Vac. Sci. Technol., B 1997, 15, 1419-1424.

(123) Grim, P. C. M.; De Feyter, S.; Gesquière, A.; Vanoppen, P.; Rücker, M.; Valiyaveettil, S.; Moessner, G.; Müllen, K.; De Schryver, F. C. Angew. Chem., Int. Edit. 1997, 36, 2601-2603.

(124) Venkataraman, B.; Flynn, G. W. Atomic Force Microscopy/Scanning Tunneling Microscopy 2, [Proceedings of the U.S. Army Soldier Systems Command, Natick Research, Development and Engineering Center Atomic Force Microscopy/Scanning Tunneling Microscopy (AFM/STM) Symposium], 2nd, Natick, Mass., June 7-9, 1994 1997, 83-90.

(125) Fukunaga, T.; Harada, K.; Takashima, W.; Kaneto, K. Jpn. J. Appl. Phys., Part 1 1997, 36, 4466-4467.

(126) Eichhorst-Gerner, K.; Stabel, A.; Moessner, G.; Declerq, D.; Valiyaveettil, S.; Enkelmann, V.; Müllen, K.; Rabe, J. P. Angew. Chem., Int. Edit. 1996, 35, 1492-1495.

(127) Stevens, F.; Dyer, D. J.; Müller, U.; Walba, D. M. Langmuir 1996, 12, 5625-5629.

(128) Cyr, D. M.; Venkataraman, B.; Flynn, G. W.; Black, A.; Whitesides, G. M. J. Phys. Chem. 1996, 100, 13747-13759.

(129) Vanoppen, P.; Grim, P. C. M.; Rücker, M.; De Feyter, S.; Moessner, G.; Valiyaveettil, S.; Müllen, K.; De Schryver, F. C. J. Phys. Chem. 1996, 100, 19636-19641.

(130) Stevens, F.; Dyer, D. J.; Walba, D. M. Langmuir 1996, 12, 436-440.

(131) Stevens, F.; Dyer, D. J.; Walba, D. M. Angew. Chem., Int. Edit. 1996, 35, 900-901.

(132) Gabriel, J. C.; Larsen, N. B.; Larsen, M.; Harrit, N.; Pedersen, J. S.; Schaumburg, K.; Bechgaard, K. Langmuir 1996, 12, 1690-1692.

(133) Walba, D. M.; Stevens, F.; Dyer, D. J. Polym. Prepr. (Am. Chem. Soc., Div. Polym. Chem.) 1996, 37, 489-490.

(134) Walzer, K.; Hietschold, M. J. Vac. Sci. Technol., B 1996, 14, 1461-1465.

(135) Müller, H.; Petersen, J.; Strohmaier, R.; Gompf, B.; Eisenmenger, W.; Vollmer, M. S.; Effenberger, F. Adv. Mater. 1996, 8, 733-737.

(136) Bäuerle, P.; Fischer, T.; Bidlingmeier, B.; Stabel, A.; Rabe, J. P. Angew. Chem., Int. Edit. 1995, 34, 303-307.

(137) Yoshimura, K.; Arakawa, H.; Ikai, A. Jpn. J. Appl. Phys., Part 1 1995, 34, 3368-3372.

(138) Stabel, A.; Heinz, R.; Rabe, J. P.; Wegner, G.; De Schryver, F. C.; Corens, D.; Dehaen, W.; Süling, C. J. Phys. Chem. 1995, 99, 8690-8697.

(139) Hibino, M.; Sumi, A.; Hatta, I. Jpn. J. Appl. Phys., Part 1 1995, 34, 3354-3359.

(140) Stabel, A.; Dasaradhi, L.; O'Hagan, D.; Rabe, J. P. Langmuir 1995, 11, 1427-1430. 
(141) Fritz, J.; Probst, O.; Dey, S.; Grafström, S.; Kowalski, J.; Neumann, R. Surf. Sci. 1995, 329, L613-L618.

(142) Canet, D.; Guillain, F.; Sanson, A. J. Trace Microprobe Tech. 1995, 13, 361-362.

(143) Hatta, I.; Nishino, J.; Sumi, A.; Hibino, M. Jpn. J. Appl. Phys., Part 1 1995, 34, 3930-3936.

(144) Heinz, R.; Rabe, J. P.; Meister, W.-V.; Hoffmann, S. Thin Solid Films 1995, 264, 246-249.

(145) Venkataraman, B.; Breen, J. J.; Flynn, G. W. J. Phys. Chem. 1995, 99, 6608-6619.

(146) Venkataraman, B.; Flynn, G. W.; Wilbur, J. L.; Folkers, J. P.; Whitesides, G. M. J. Phys. Chem. 1995, 99, 8684-8689.

(147) Walba, D. M.; Stevens, F.; Parks, D. C.; Clark, N. A.; Wand, M. D. Science 1995, 267, $1144-$ 1147.

(148) Liu, T. L.; Parakka, J. P.; Cava, M. P.; Kim, Y. T. Langmuir 1995, 11, 4205-4208.

(149) Tao, N. J.; Cardenas, G.; Cunha, F.; Shi, Z. Langmuir 1995, 11, 4445-4448.

(150) Patrick, D. L.; Beebe, T. P. Langmuir 1994, 10, 298-302.

(151) Gunning, A. P.; Kirby, A. R.; Mallard, X.; Morris, V. J. J. Chem. Soc., Faraday Trans. 1994, 90, 2551-2554.

(152) Couto, M. S.; Liu, X. Y.; Meekes, H.; Bennema, P. J. Appl. Phys. 1994, 75, 627-628.

(153) Stabel, A.; Rabe, J. P. Synth. Met. 1994, 67, 47-53.

(154) Elbel, N.; Roth, W.; Günther, E.; von Seggern, H. Surf. Sci. 1994, 303, 424-432.

(155) Heinz, R.; Stabel, A.; Rabe, J. P.; Wegner, G.; De Schryver, F. C.; Corens, D.; Dehaen, W.; Süling, C. Angew. Chem., Int. Edit. 1994, 33, 2080-2083.

(156) Freund, J.; Probst, O.; Grafström, S.; Dey, S.; Kowalski, J.; Neumann, R.; Wörtge, M.; zu Putlitz, G. J. Vac. Sci. Technol., B 1994, 12, 1914-1917.

(157) Brandow, S. L.; Harrison, J. A.; DiLella, D. P.; Colton, R. J.; Pfeiffer, S.; Shashidhar, R. Liq. Cryst. 1993, 13, 163-170.

(158) Wawkuschewski, A.; Cantow, H.-J.; Magonov, S. N.; Möller, M.; Liang, W.; Whangbo, M.-H. Adv. Mater. 1993, 5, 821-826.

(159) Liang, W.; Whangbo, M.-H.; Wawkuschewski, A.; Cantow, H.-J.; Magonov, S. N. Adv. Mater. 1993, 5, 817-821.

(160) Grafström, S.; Probst, O.; Dey, S.; Freund, J.; Kowalski, J.; Neumann, R.; Wörtge, M.; Zu Putlitz, G. Proc. SPIE-Int. Soc. Opt. Eng. 1993, 1891, 56-65.

(161) Yeo, Y. H.; McGonigal, G. C.; Thomson, D. J. Langmuir 1993, 9, 649-651.

(162) Dai, P.; Wang, S.-K.; Taub, H.; Buckley, J. E.; Ehrlich, S. N.; Larese, J. Z.; Binnig, G.; Smith, D. P. E. Phys. Rev. B 1993, 47, 7401-7407. 
(163) Parks, D. C.; Clark, N. A.; Walba, D. M.; Beale, P. D. Phys. Rev. Lett. 1993, 70, 607-610.

(164) Bucher, J.-P.; Roeder, H.; Kern, K. Surf. Sci. 1993, 289, 370-380.

(165) Rabe, J. P.; Buchholz, S.; Askadskaya, L. Synth. Met. 1993, 54, 339-349.

(166) Rabe, J. P.; Buchholz, S.; Askadskaya, L. Phys. Scr. 1993, T49A, 260-263.

(167) Askadskaya, L.; Boeffel, C.; Rabe, J. P. Ber. Bunsen.-Ges. 1993, 97, 517-521.

(168) Buchholz, S.; Rabe, J. P. Angew. Chem., Int. Edit. 1992, 31, 189-191.

(169) Smith, D. P. E.; Heckl, W. M.; Klagges, H. A. Surf. Sci. 1992, 278, 166-174.

(170) Askadskaya, L.; Rabe, J. P. Phys. Rev. Lett. 1992, 69, 1395-1398.

(171) Rabe, J. P.; Buchholz, S. Makromol. Chem., Macromol. Symp. 1991, 50, 261-268.

(172) McMaster, T. J.; Carr, H.; Miles, M. J.; Cairns, P.; Morris, V. J. Liq. Cryst. 1991, 9, 11-18.

(173) Rabe, J. P.; Buchholz, S. Science 1991, 253, 424-427.

(174) Mizutani, W.; Shigeno, M.; Ohmi, M.; Suginoya, M.; Kajimura, K.; Ono, M. J. Vac. Sci. Technol., B 1991, 9, 1102-1106.

(175) Buchholz, S.; Rabe, J. P. J. Vac. Sci. Technol., B 1991, 9, 1126-1128.

(176) Brandow, S. L.; DiLella, D. P.; Colton, R. J.; Shashidhar, R. J. Vac. Sci. Technol., B 1991, 9, 1115-1118.

(177) Smith, D. P. E.; Hörber, J. K. H.; Binnig, G.; Nejoh, H. Nature 1990, 344, 641-644.

(178) Bernhardt, R. H.; McGonigal, G. C.; Schneider, R.; Thomson, D. J. J. Vac. Sci. Technol., A 1990, 8, 667-671.

(179) Spong, J. K.; Mizes, H. A.; Lacomb, L. J.; Dovek, M. M.; Frommer, J. E.; Foster, J. S. Nature 1989, 338, 137-139.

(180) Wu, X.-L.; Lieber, C. M. J. Phys. Chem. 1988, 92, 5556-5557. 
6) The structural assignments for high and medium resolution entries into the 2DSD.

Record 1
13-hydroxytridecanenitrile
crystal class

\section{Record 2}

1-decanol

$\begin{array}{ll}\text { plane group } & p g \\ \text { crystal class } & \text { rectangular } \\ Z & 2 \\ Z^{\prime} & 1 \\ \text { molecular } & \mathrm{C}(\mathrm{s}) \\ \text { symmetry } & \end{array}$

symmetry

site symmetry 1

molecular $\quad 158.28$

weight (Da)

multicomponent no

Claypool, C. L., Faglioni, F., Goddard, W. A., Gray, H. B., Lewis, N. S. \& Marcus, R. A. "Source of image contrast in STM images of functionalized alkanes on graphite: A systematic functional group approach." J. Phys. Chem. B 1997 101, 5978-5995.

\section{Record 3}

4-n-octyloxy-4'-cyanobiphenyl (8OCB)

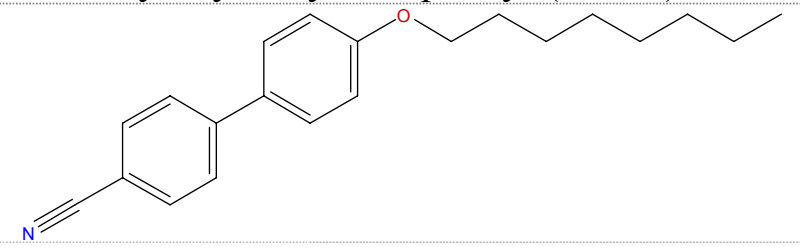

plane group pg

crystal class rectangular

Z

$\begin{array}{ll}Z^{\prime} & 1 \\ \begin{array}{l}\text { molecular } \\ \text { symmetry }\end{array} & \mathrm{C}(\mathrm{s}) \\ \text { site symmetry } & 1 \\ \begin{array}{l}\text { molecular } \\ \text { weight }(\text { Da) }\end{array} & 307.43 \\ \text { multicomponent } & \text { no }\end{array}$

Walzer, K. \& Hietschold, M. J. Vac. Sci. Technol., B 1996 14, 1461-1465.

\section{Record 4}

\section{1-tetradecanol}

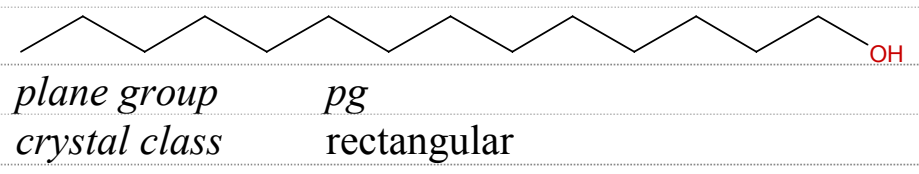

crystal class rectangular

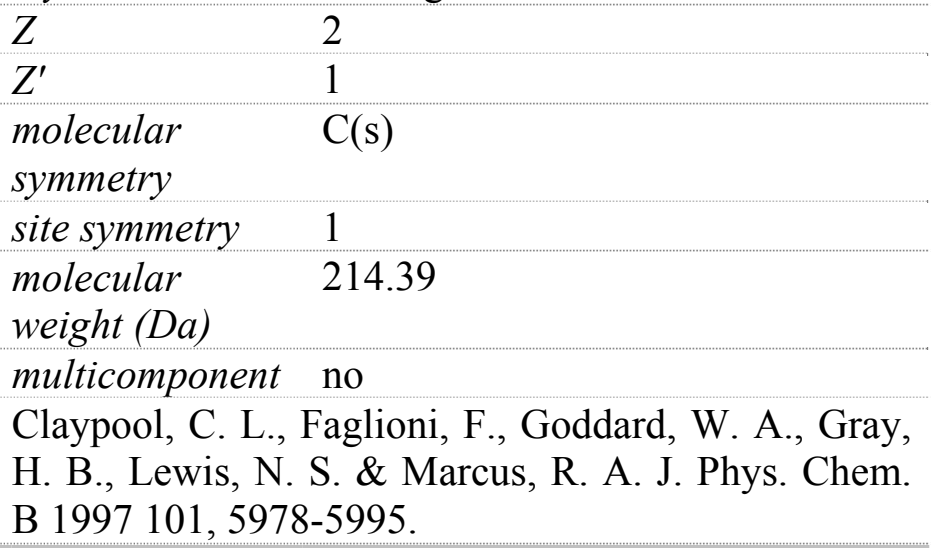

\section{Record 5}

12,12,12-trifluorododecan-1-ol

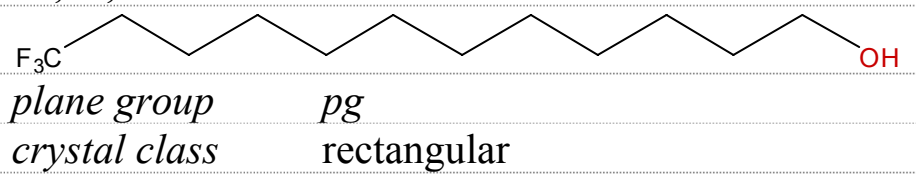

$Z$ 2

$Z^{\prime} \quad 1$

molecular $\quad \mathrm{C}(\mathrm{s})$

symmetry

site symmetry 1

molecular $\quad 240.31$

weight (Da)

multicomponent no

Claypool, C. L., Faglioni, F., Goddard, W. A., Gray, H. B., Lewis, N. S. \& Marcus, R. A. J. Phys. Chem.

B 1997 101, 5978-5995.

\section{Record 6}

12-bromododecanol

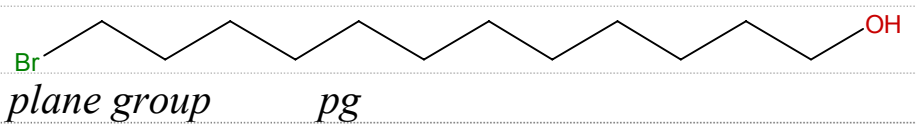




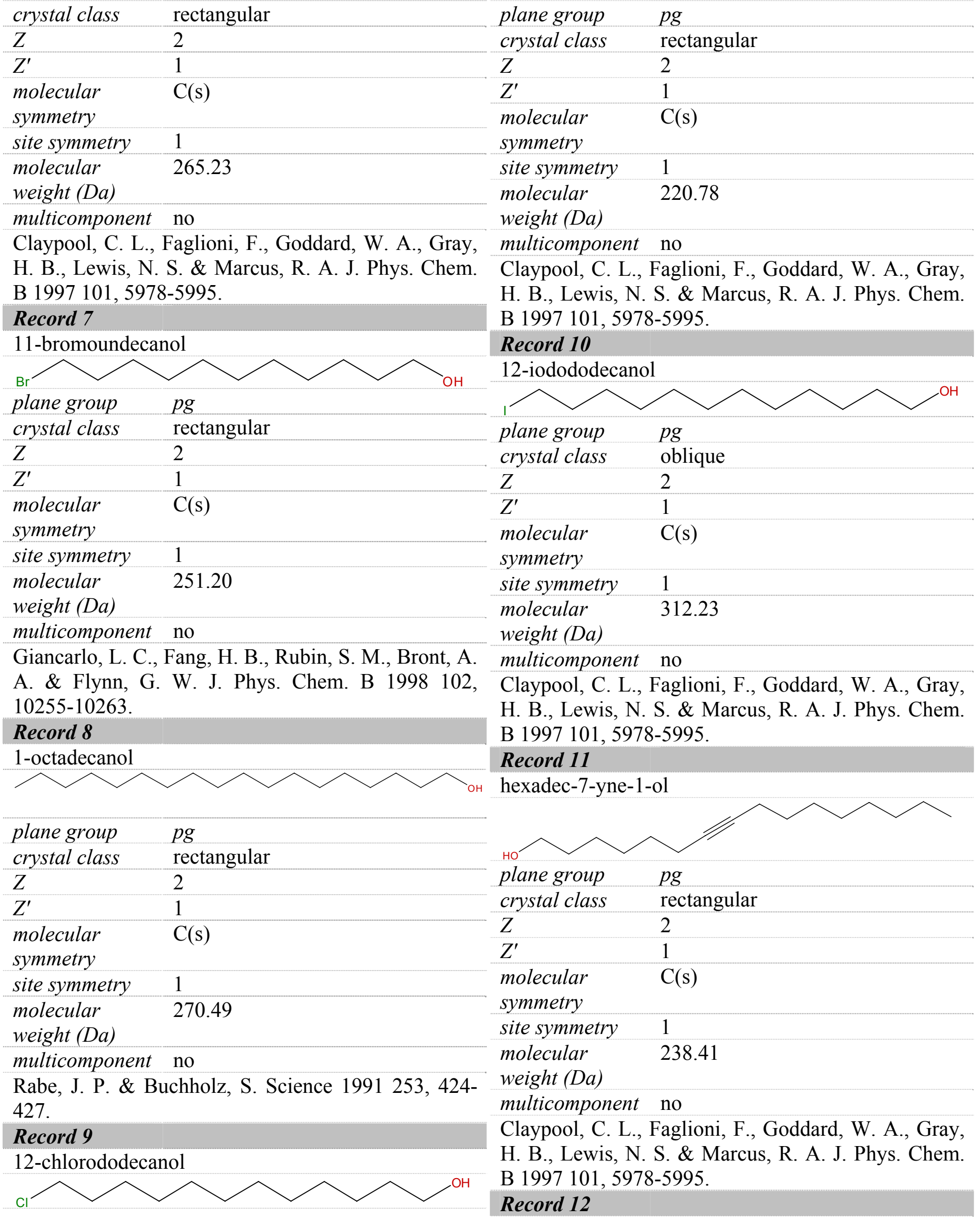


3-n-octadecylthiophene

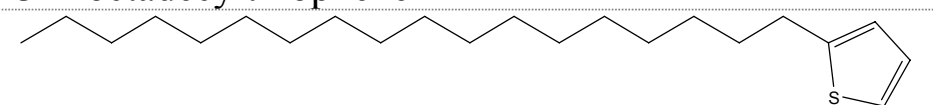

plane group pg

crystal class rectangular

$\begin{array}{ll}Z & 2 \\ Z^{\prime} & 1\end{array}$

molecular $\mathrm{C}(\mathrm{s})$

symmetry

site symmetry 1

molecular $\quad 336.62$

weight $(D a)$

multicomponent no

Fukunaga, T., Harada, K., Takashima, W. \& Kaneto, K. Jpn. J. Appl. Phys., Part 11997 36, 4466-4467.

\section{Record 13}

octadecyl undecyl disulfide

$\begin{array}{ll}\text { plane group } & p g \\ \text { crystal class } & \text { rectangular } \\ Z & 2 \\ Z^{\prime} & 1\end{array}$

molecular $\mathrm{C}(\mathrm{s})$

symmetry

$\begin{array}{ll}\text { site symmetry } & 1 \\ \text { molecular } & 472.92\end{array}$

weight (Da)

multicomponent no

noh, J., Lee, D., Hara, M., Lee, H., Sasabe, H. \&

Knoll, W. Jpn. J. Appl. Phys., Part 11999 38, 38973900.

\section{Record 14}

1-dodecanol

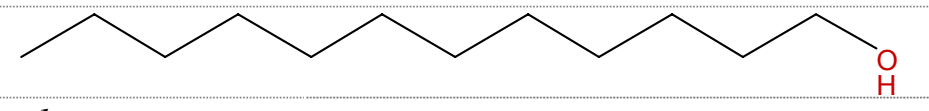

plane group pg

crystal class rectangular

$Z \quad 2$

Zprime

molecular

$\mathrm{C}(\mathrm{s})$

symmetry

site symmetry 1

molecular

186.33

weight (Da)

multicomponent no
Claypool, C. L., Faglioni, F., Goddard, W. A., Gray, H. B., Lewis, N. S. \& Marcus, R. A. J. Phys. Chem.

B 1997 101, 5978-5995.

\section{Record 15}

1-docosanol

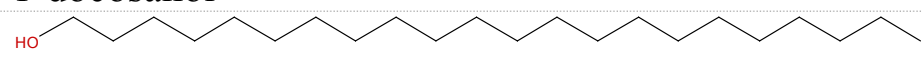

plane group pg

crystal class rectangular

$\begin{array}{ll}Z & 2 \\ Z^{\prime} & 1\end{array}$

molecular $\quad \mathrm{C}(\mathrm{s})$

symmetry

site symmetry 1

molecular $\quad 326.60$

weight (Da)

multicomponent no

Gunning, A. P., Kirby, A. R., Mallard, X. \& Morris,

V. J. J. Chem. Soc., Faraday Trans. 1994 90, 25512554.

\section{Record 16}

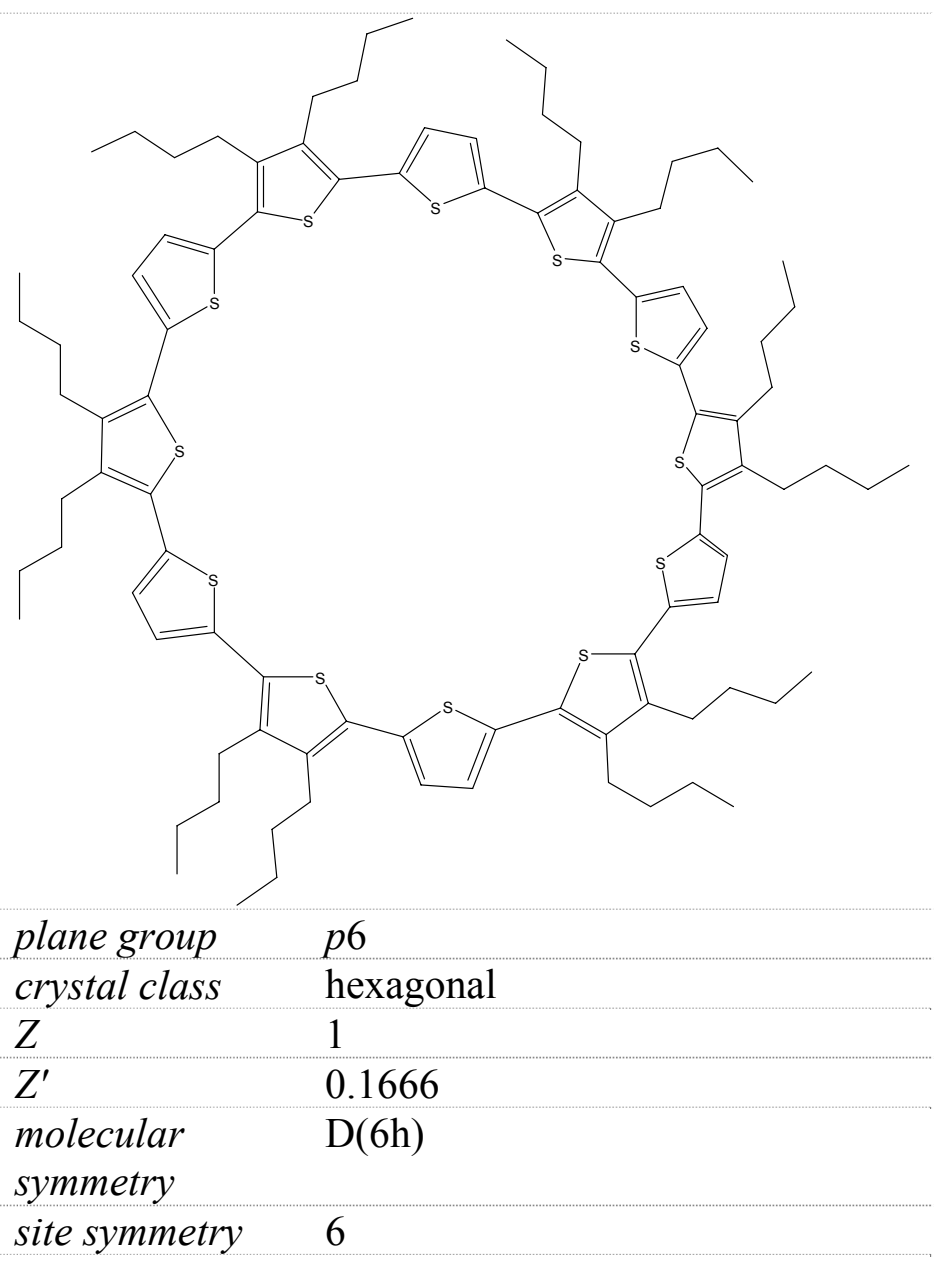


molecular

1658.76

weight (Da)

multicomponent no

Krömer, J., Rios-Carreras, I., Furhmann, G., Musch, C., Wunderlin, M., Debaerdemacker, T., MenaOsteritz, E. \& Bäuerle, P. Angew. Chem., Int. Edit. 2000 39, 3481-3486.

\section{Record 17}

6-(4-(4-(2,5-bis((S)-2-methylbutoxy)-4-(3,4,5-

tris(dodecyloxy)styryl)styryl)-2,5-bis((S)-2-

methylbutoxy)styryl)phenyl)-1,3,5-triazine-2,4-

diamine

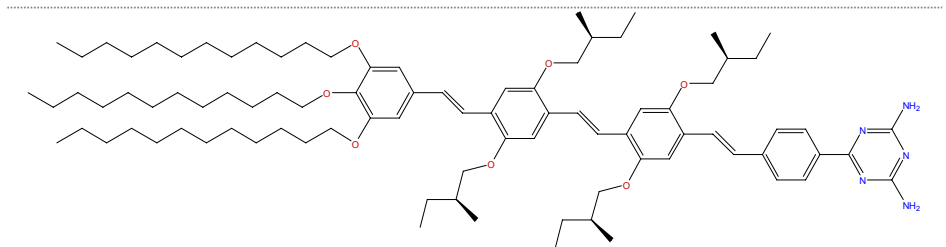

plane group $\quad p 6$

crystal class hexagonal

$Z^{\prime}$

molecular

$\mathrm{C}(1)$

symmetry

site symmetry 1

molecular

1391.09

weight (Da)

multicomponent no

Jonkheijm, P., Miura, A., Zdanowska, M., Hoeben, F. J. M., De Feyter, S., Schenning, A. P. H. J., De Schryver, F. C. \& Meijer, E. W. Angew. Chem., Int. Edit. 2004 43, 74-78.

\section{Record 18}

hexadodecyl-hexaphenylbenzene

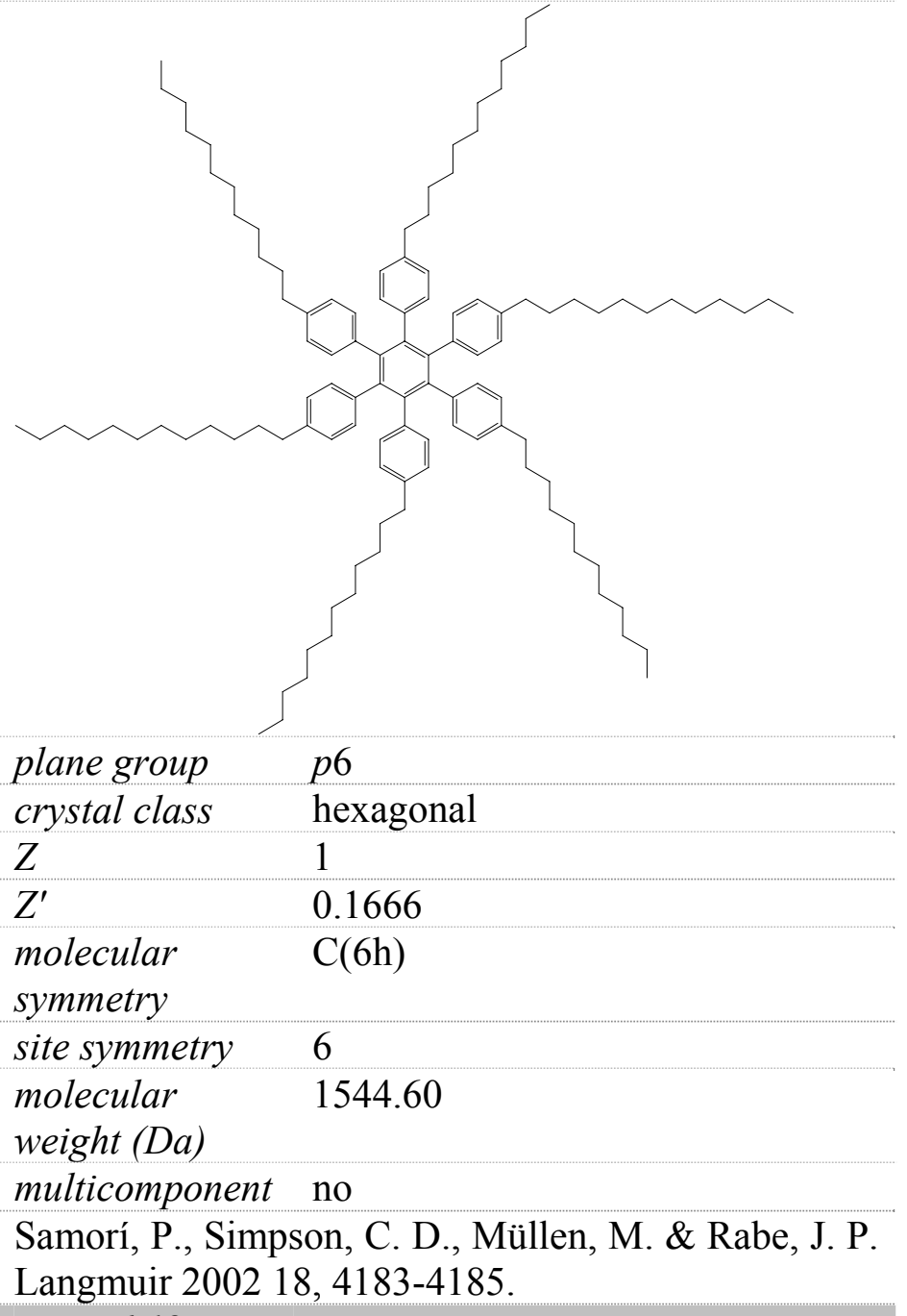

\section{Record 19}

2,5,8,11,14,17-hexakis((R)-3,7-

dimethyloctyl)hexabenzo[bc,ef,hi,kl,no,qr]coronene

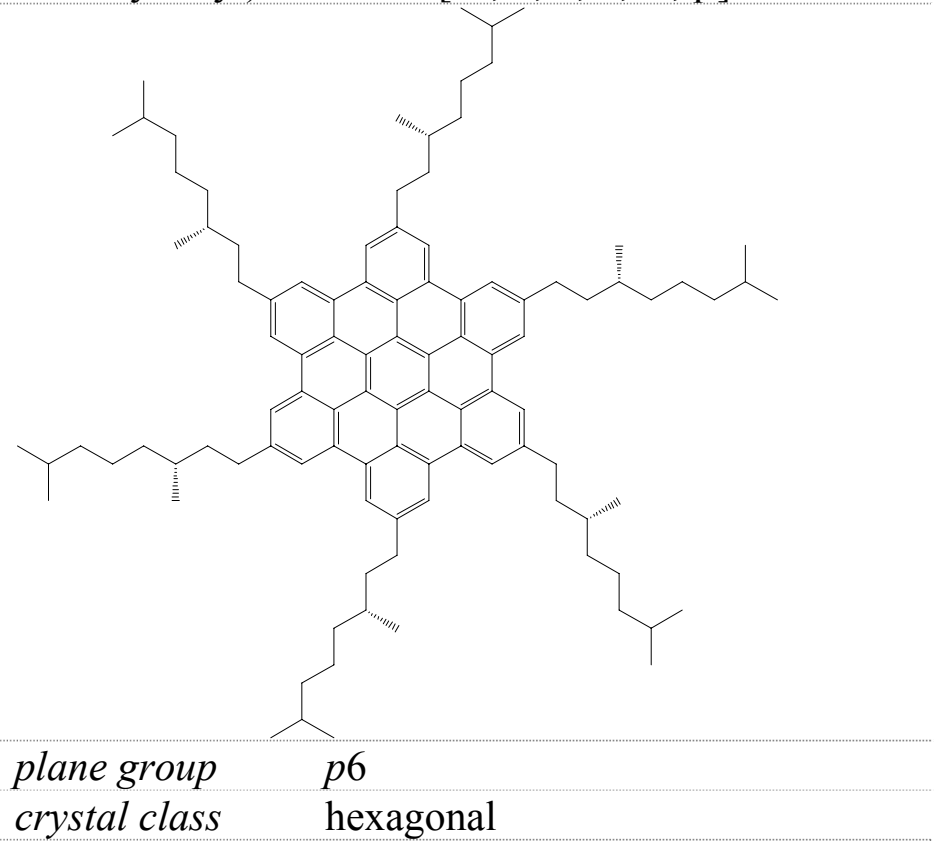




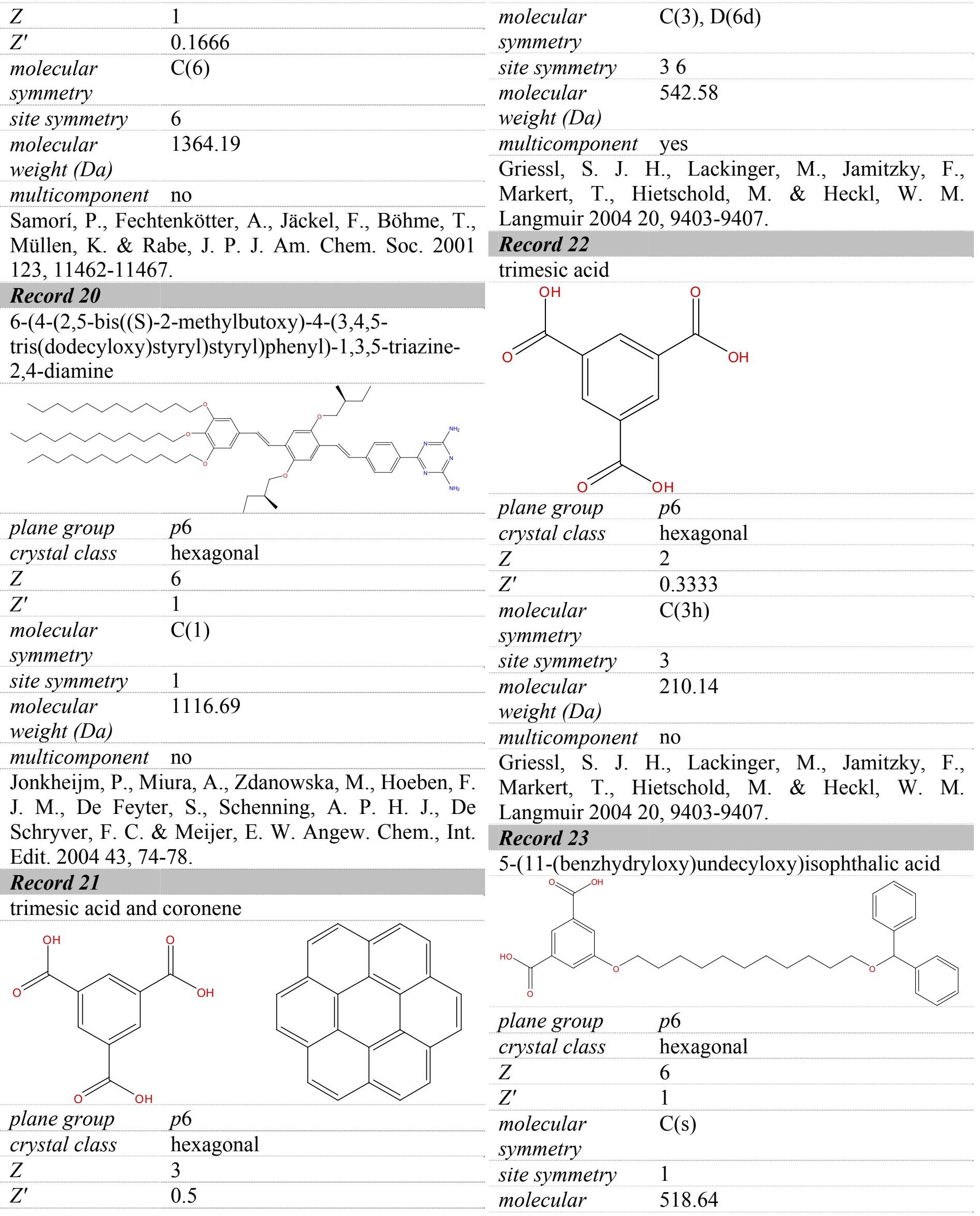


weight (Da)

multicomponent no

De Feyter, S., Gesquière, A., Klapper, M., Müllen, K. \& De Schryver, F. C. Nano Lett. 2003 3, 1485-1488.

\section{Record 24}

hexakis-2,3,6,7,10,11-pentyloxytriphenylene

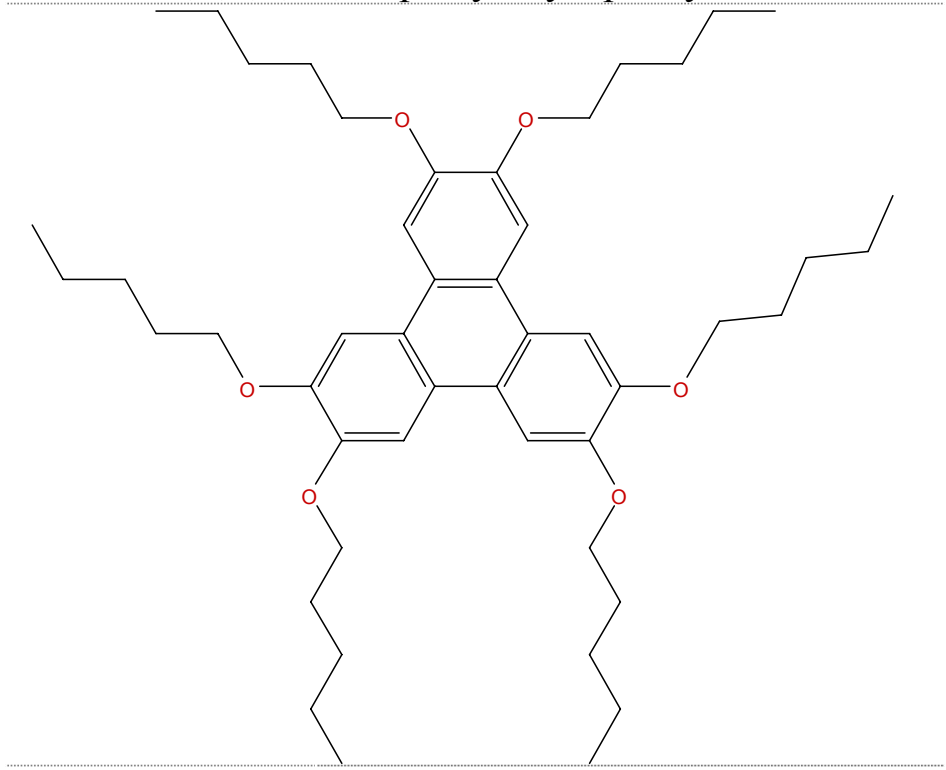

plane group p3

crystal class hexagonal

$\begin{array}{ll}Z & 1 \\ Z^{\prime} & 0.3333\end{array}$

molecular $\mathrm{D}(3 \mathrm{~h})$

symmetry

site symmetry 3

molecular $\quad 745.08$

weight $(\mathrm{Da})$

multicomponent no

Charra, F. \& Cousty, J. Opt. Mater. 1998 9, 386-389.

Record 25

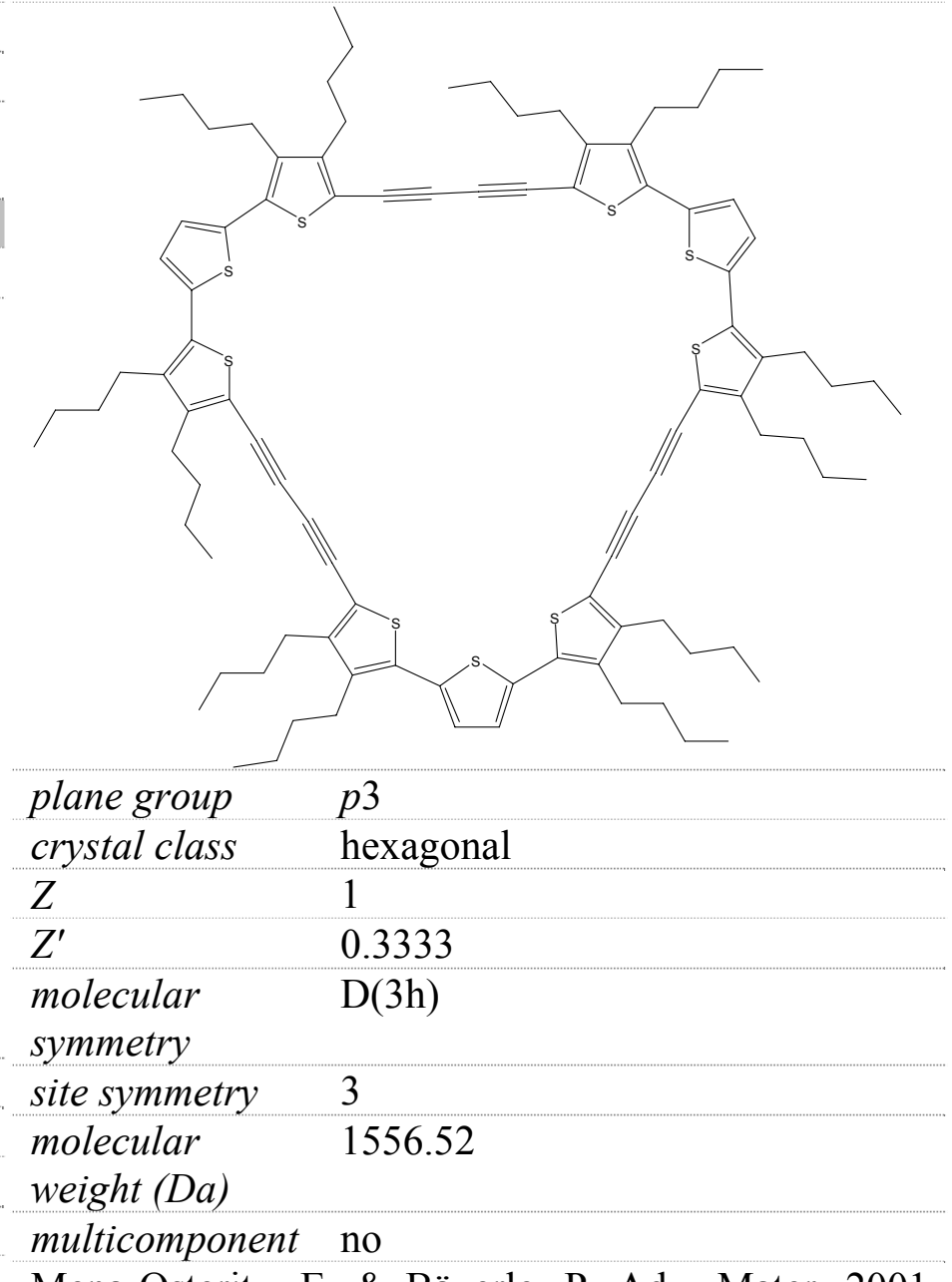

Mena-Osteritz, E. \& Bäuerle, P. Adv. Mater. 2001 13, 243-246.

Record 26

hexakis-2,3,6,7,10,11-nonyloxytriphenylene 


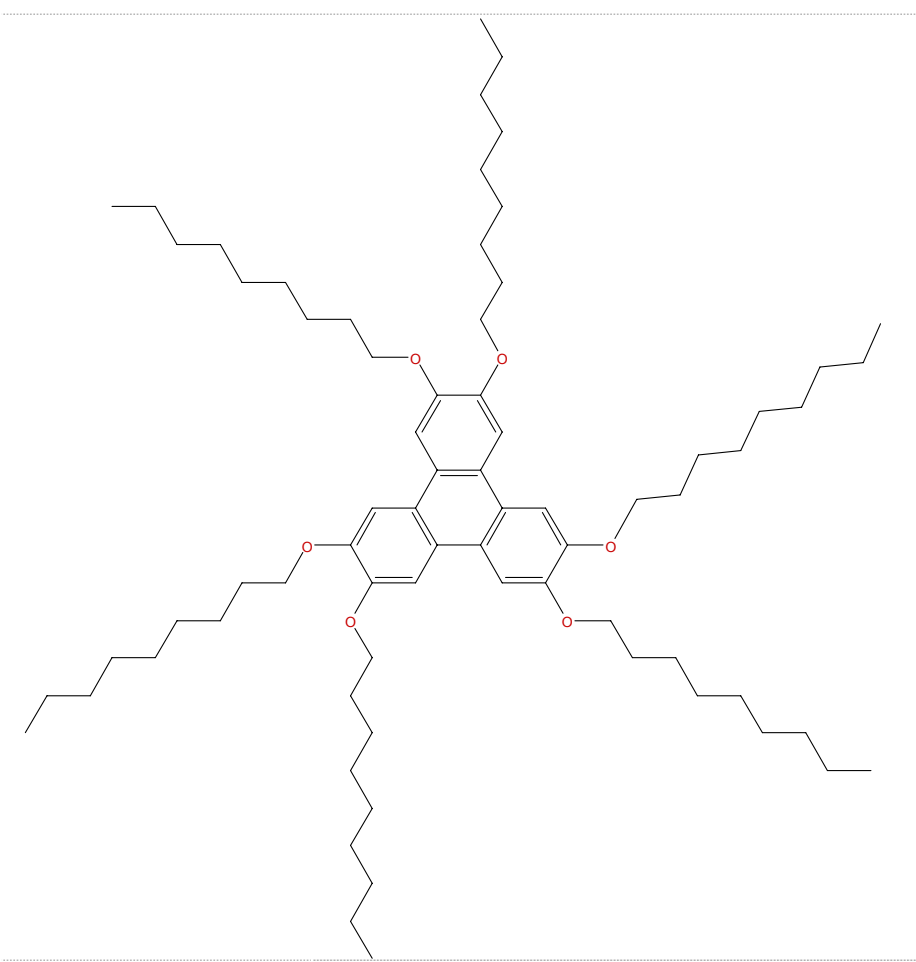

plane group p3

crystal class hexagonal

$\begin{array}{ll}Z & 3 \\ Z^{\prime} & 1\end{array}$

molecular $\quad \mathrm{D}(3 \mathrm{~h})$

symmetry

site symmetry 333

molecular $\quad 1081.72$

weight (Da)

multicomponent no

Charra, F. \& Cousty, J. Phys. Rev. Lett. 1998 80, 1682-1685.

\section{Record 27}

2,3,6,7,12,13,16,17,22,23,26,27-

Dodecakis(decyloxy)hexabenzo[a,c,k,m,u,w]trinapht hylene (4c).

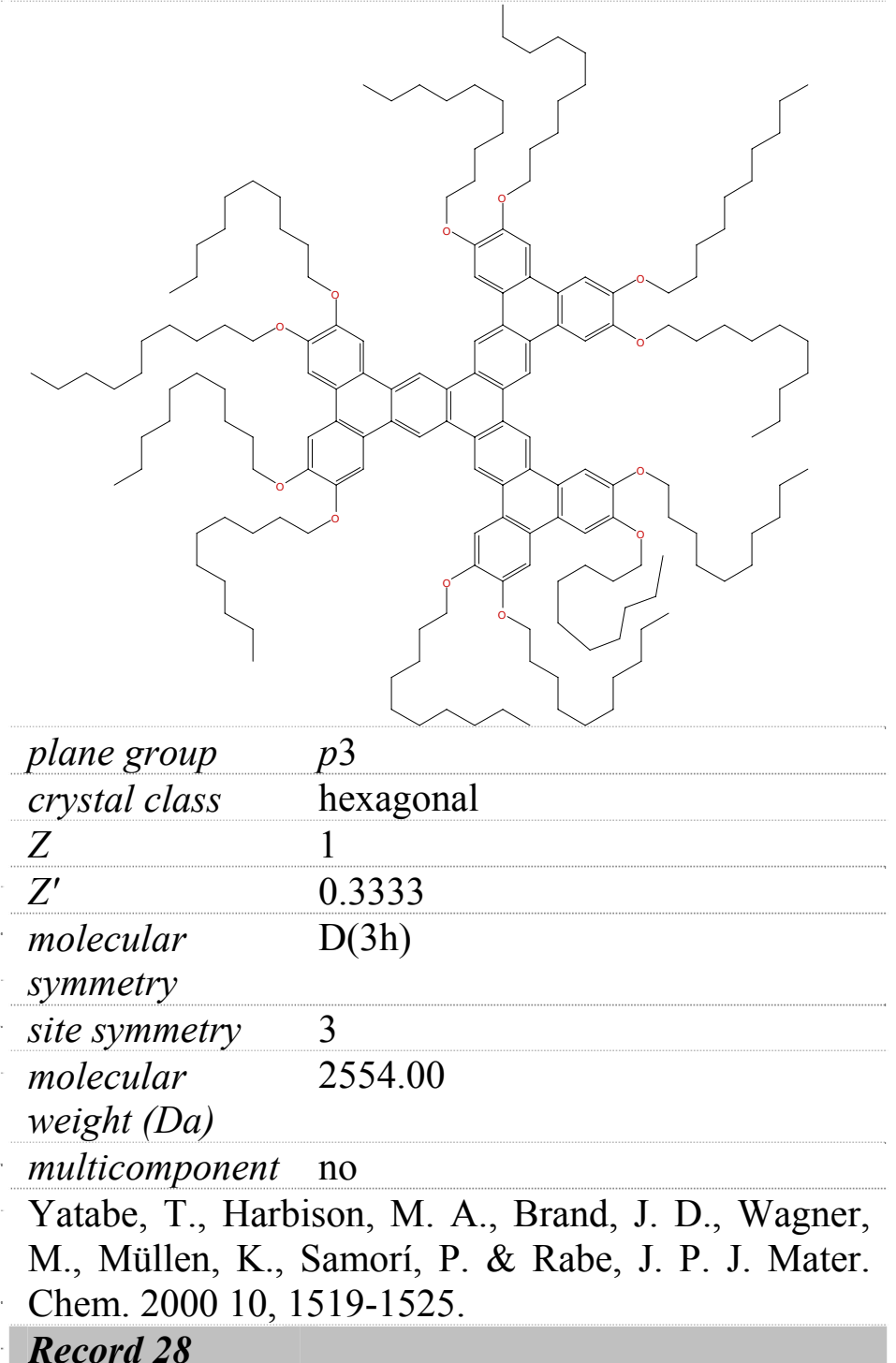

Record 28

2,3,6,7,10,11-hexakis-undecalkoxytriphenylene

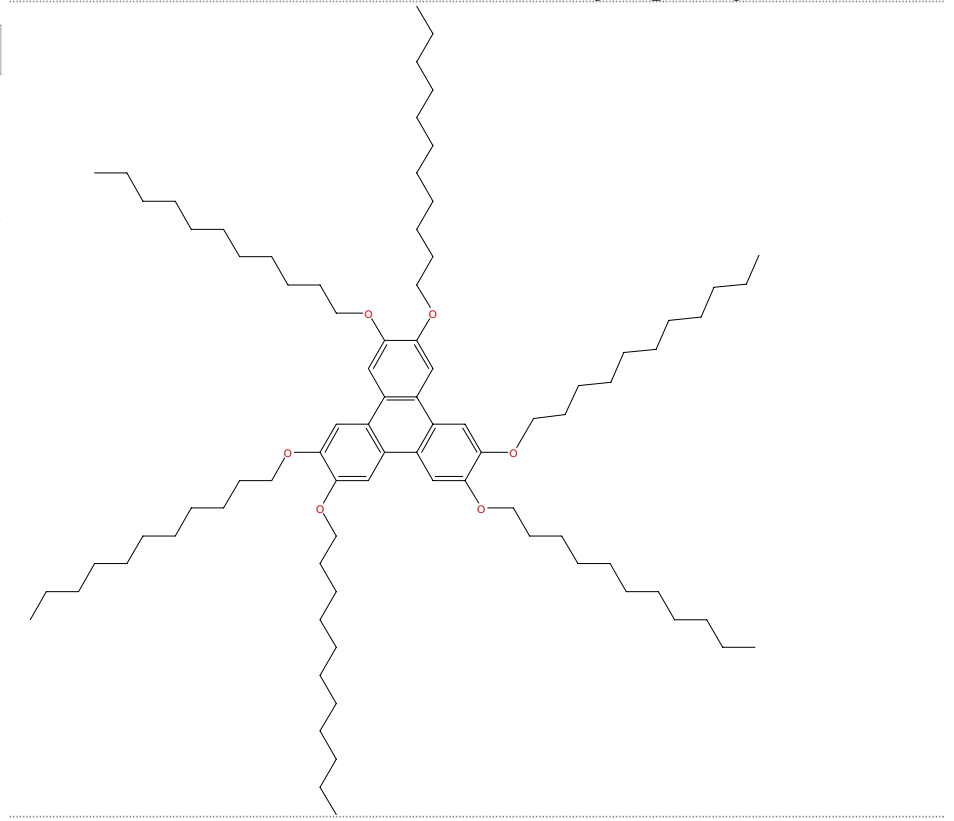




\begin{tabular}{|c|c|}
\hline plane group & p3 \\
\hline crystal class & hexagonal \\
\hline$Z$ & 3 \\
\hline$Z^{\prime}$ & 1 \\
\hline $\begin{array}{l}\text { molecular } \\
\text { symmetry }\end{array}$ & $\mathrm{D}(3 \mathrm{~h})$ \\
\hline site symmetry & 333 \\
\hline $\begin{array}{l}\text { molecular } \\
\text { weight }(D a)\end{array}$ & 1250.04 \\
\hline multicomponent & no \\
\hline
\end{tabular}

\section{Record 29}

1,2-dihexadecyldisulfane

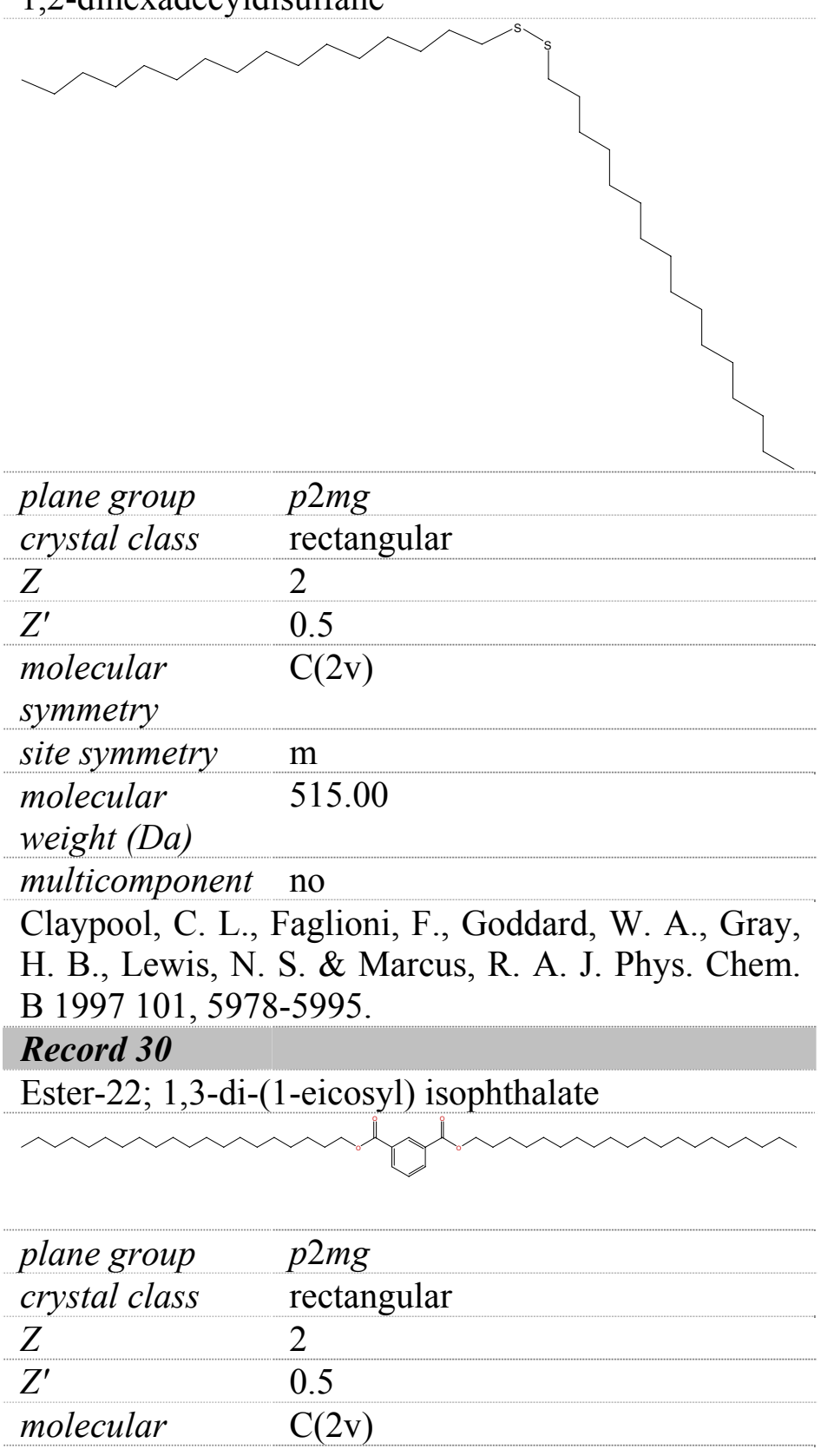

symmetry

site symmetry $\mathrm{m}$

molecular $\quad 727.19$

weight (Da)

multicomponent no

Plass, K. E., Kim, K. \& Matzger, A. J. J. Am. Chem.

Soc. 2004 126, 9042-9053.

Record 31

Ester-20; 1,3-di-(1-octadecyl) isophthalate

plane group $\quad p 2 m g$

crystal class rectangular

Z 2

$Z^{\prime} \quad 0.5$

molecular $\quad \mathrm{C}(2 \mathrm{v})$

symmetry

site symmetry $\mathrm{m}$

molecular $\quad 671.09$

weight (Da)

multicomponent no

Plass, K. E., Kim, K. \& Matzger, A. J. J. Am. Chem.

Soc. 2004 126, 9042-9053.

\section{Record 32}

1,14-tetradecanediol

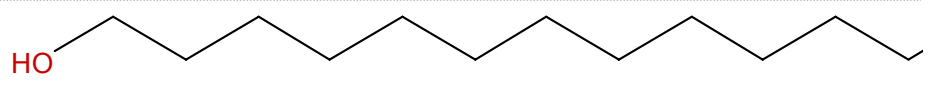

plane group $\quad p 2 g g$

crystal class rectangular

$Z \quad 2$

$Z^{\prime} \quad 0.5$

molecular $\quad \mathrm{C}(2 \mathrm{~h})$

symmetry

site symmetry 2

molecular 230.39

weight (Da)

multicomponent no

Claypool, C. L., Faglioni, F., Goddard, W. A., Gray, H. B., Lewis, N. S. \& Marcus, R. A. J. Phys. Chem. B 1997 101, 5978-5995.

\section{Record 33}

PTCD1-C4 


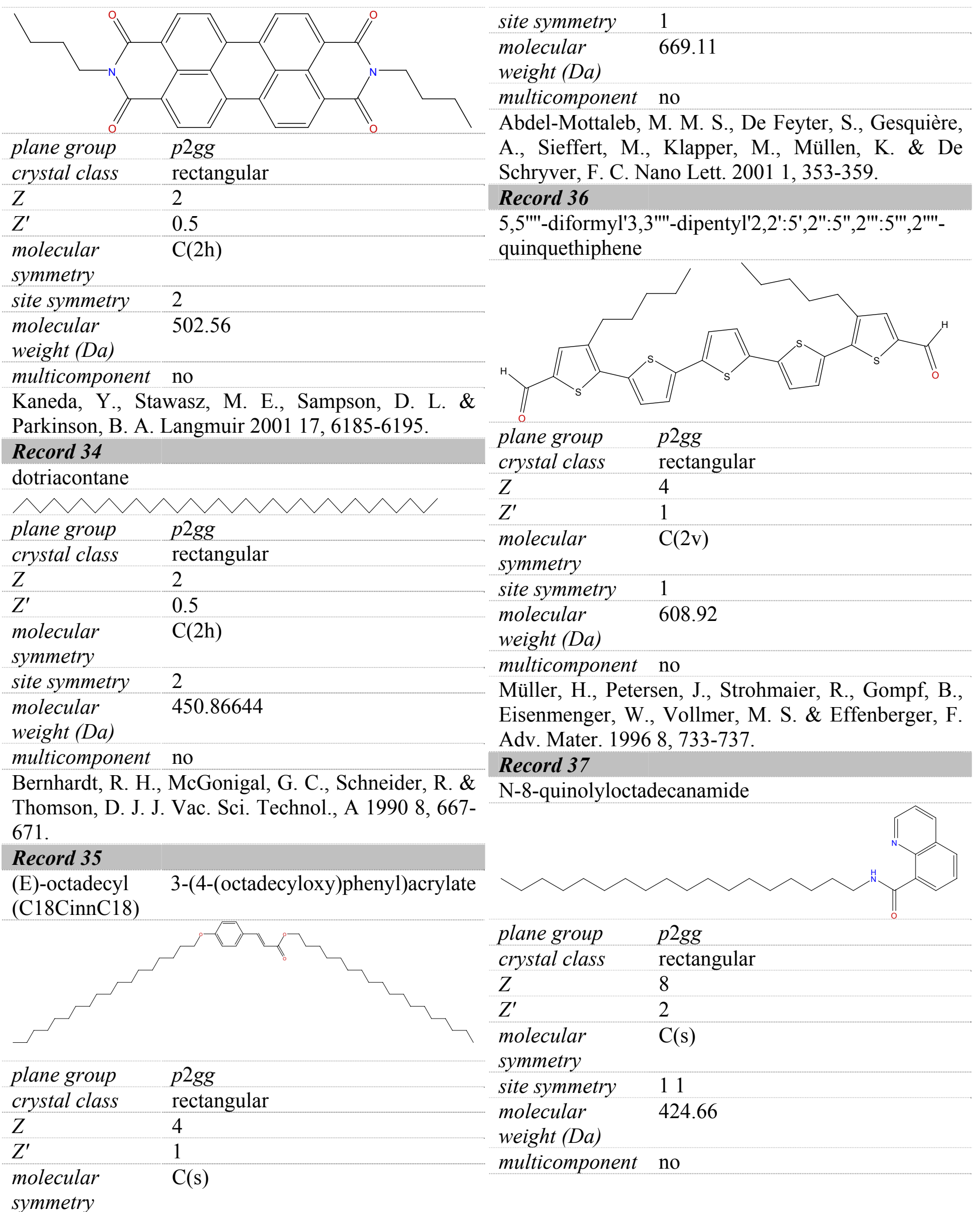


Qian, P., Nanjo, H., Yokoyama, T. \& Suzuki, T. M.

Chem. Lett. 1999 1229-1230.

\section{Record 38}

1,3,8,10-tetramethyl-N,N'-didocosyl-quinacridone

TmQA-C22 and palmitic acid

\begin{tabular}{|c|c|}
\hline plane group & $p 2 g g$ \\
\hline crystal class & rectangular \\
\hline$Z$ & 4 \\
\hline$Z^{\prime}$ & 1 \\
\hline $\begin{array}{l}\text { molecular } \\
\text { symmetry }\end{array}$ & $\mathrm{C}(\mathrm{s}), \mathrm{C}(2 \mathrm{~h})$ \\
\hline site symmetry & 12 \\
\hline $\begin{array}{l}\text { molecular } \\
\text { weight }(D a)\end{array}$ & 1242.02 \\
\hline multicomponent & yes \\
\hline
\end{tabular}

Mu, Z., Wang, Z., Zhang, X., Ye, K. \& Wang, Y. J. Phys. Chem. B 2004 108, 19955-19959.

\section{Record 39}

18-methyl pentatriacontane

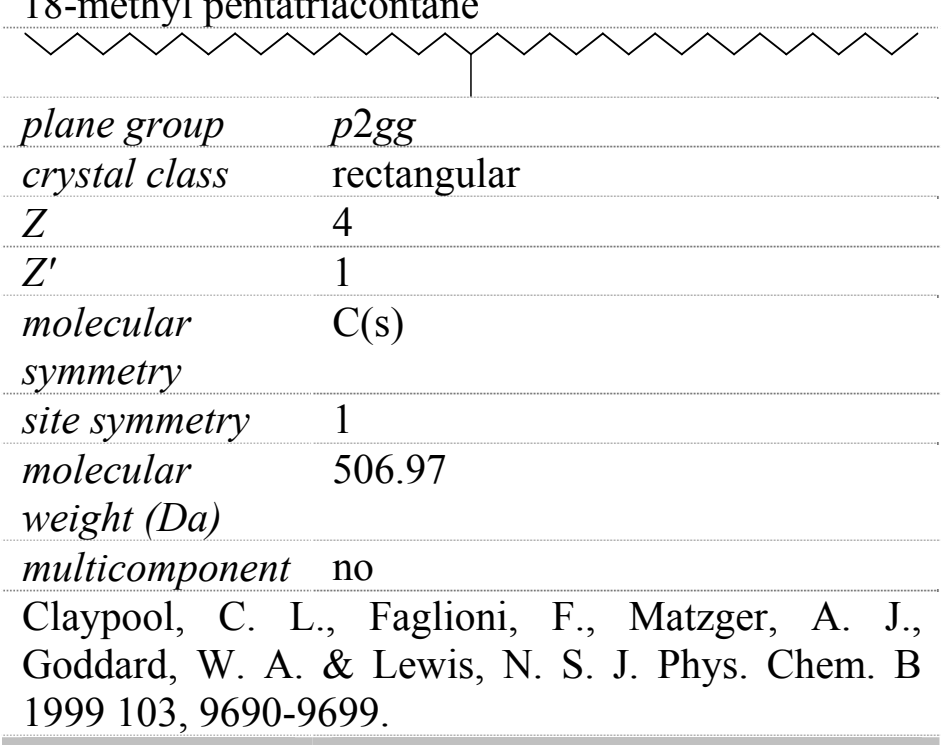

\section{Record 40}

bis(4-dibutylamino-2-hydroxyphenyl)

squaraine [4-4 SQ]
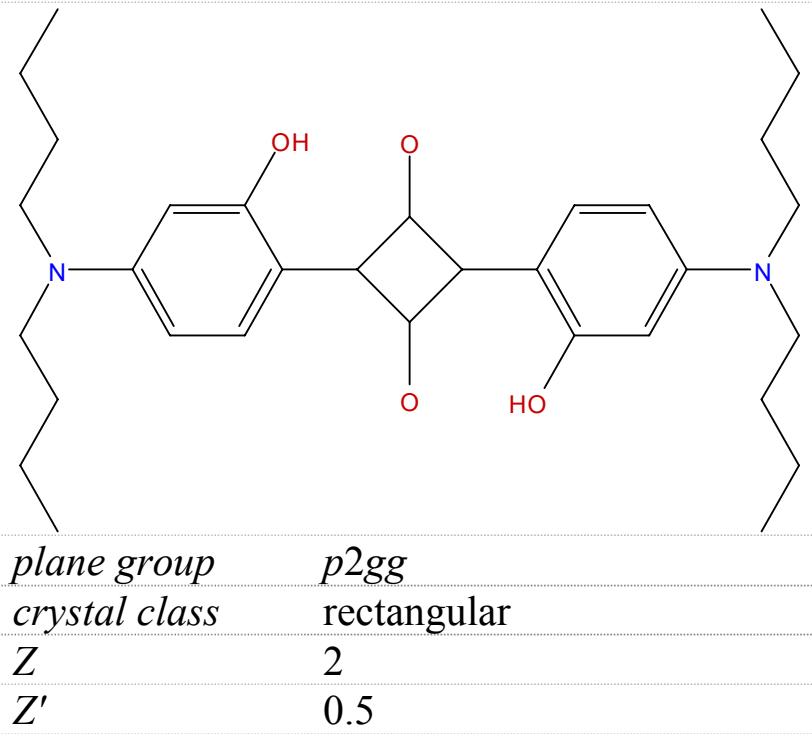

molecular

$\mathrm{C}(2 \mathrm{~h})$

symmetry

site symmetry 2

molecular $\quad 526.75$

weight (Da)

multicomponent no

Stawasz, M. E., Sampson, D. L. \& Parkinson, B. A.

Langmuir 2000 16, 2326-2342.

\section{Record 41}

$\mathrm{N}$-octadecyl-isonicotinamide

ODiN

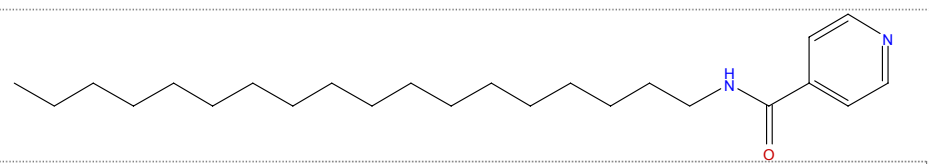

plane group $\quad$ p $2 g g$

crystal class rectangular

$Z \quad 4$

$Z^{\prime} \quad 1$

molecular $\mathrm{C}(\mathrm{s})$

symmetry

site symmetry 1

molecular $\quad 374.60$

weight (Da)

multicomponent no

Nanjo, H., Qian, P., Yokoyama, T. \& Suzuki, T. M.

Jpn. J. Appl. Phys., Part 12003 42, 6560-6563.

Record 42

1-heptadecanoic acid

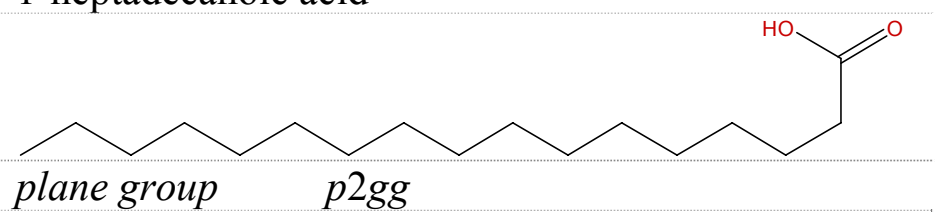




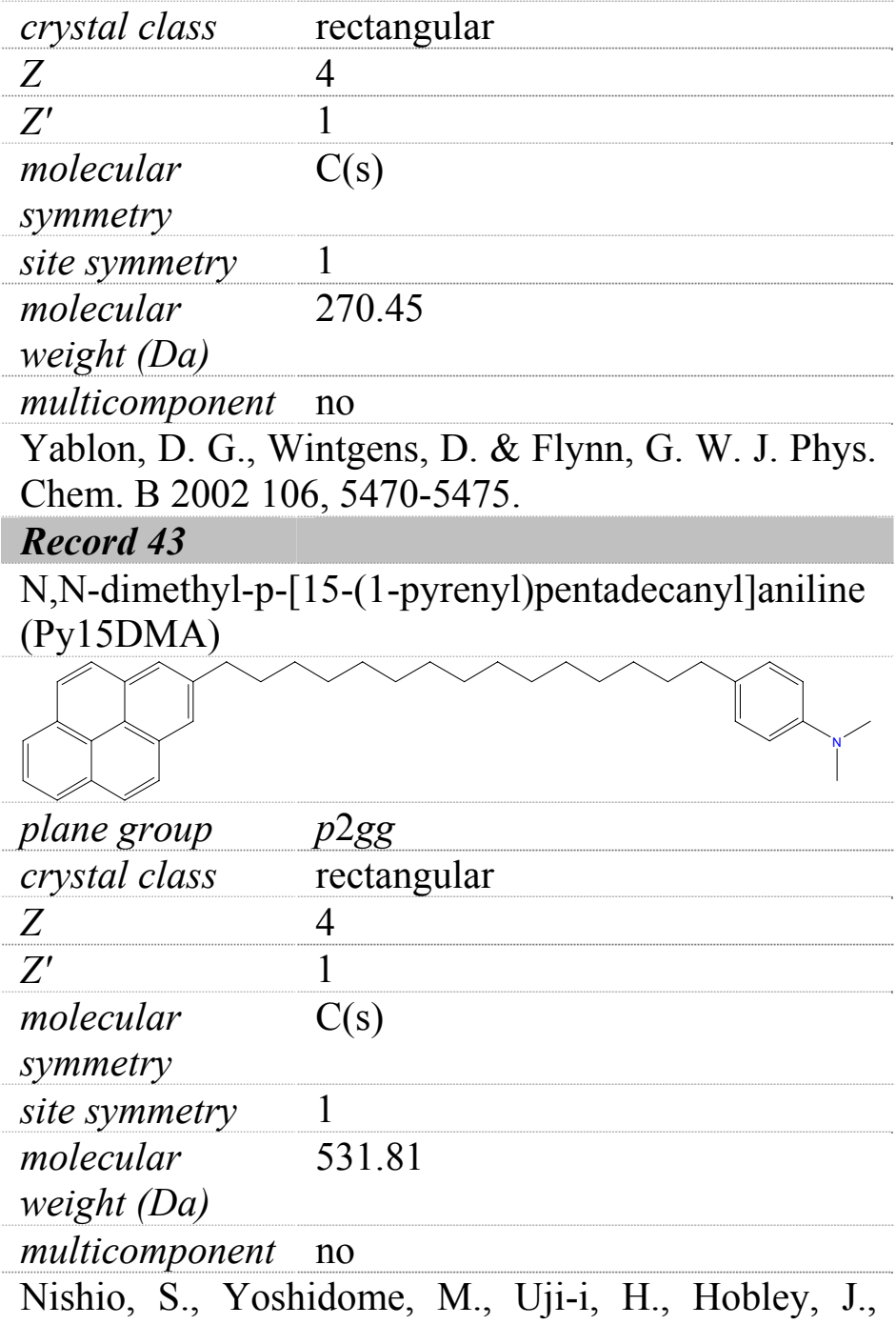
Fukumura, H. \& Zachariasse, K. A. Chem. Lett. 2004, 33, 1506-1507.

\section{Record 44}

pentadecanoic acid

plane group
crystal class

4-n-octyloxy-4'-cyanobiphenyl (8OCB)

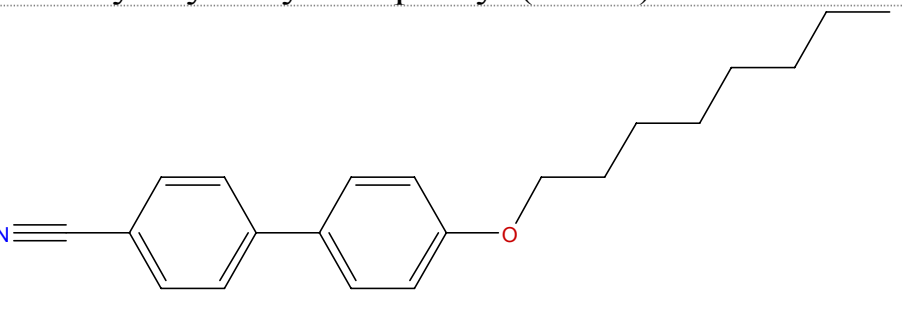

plane group $\quad p 2 g g$

crystal class rectangular

$\begin{array}{ll}Z & 4 \\ Z^{\prime} & 1 \\ \text { molecular } & \mathrm{C}(\mathrm{s}) \\ \text { symmetry } & \\ \text { site symmetry } & 1 \\ \begin{array}{l}\text { molecular } \\ \text { weight (Da) }\end{array} & 307.43 \\ \text { multicomponent } & \text { no }\end{array}$

Taki, S. \& Kai, S. Jpn. J. Appl. Phys., Part 12001 40, 4187-4192.

\section{Record 46}

4-n-nonyloxy-4'-cyanobiphenyl (9OCB)

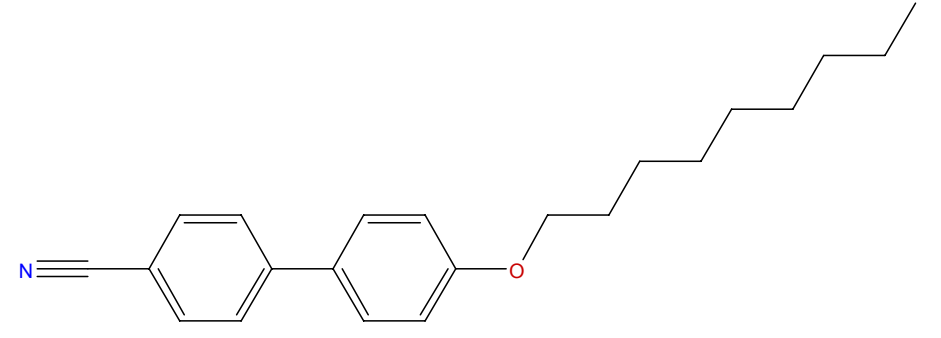

plane group $\quad p 2 g g$

crystal class rectangular

$Z \quad 4$

$Z^{\prime} \quad 1$

molecular $\mathrm{C}(\mathrm{s})$

symmetry

site symmetry 1

molecular $\quad 321.46$

weight (Da)

multicomponent no

Taki, S. \& Kai, S. Jpn. J. Appl. Phys., Part 12001 40, 4187-4192.

Record 47

12-bromododecanoic acid

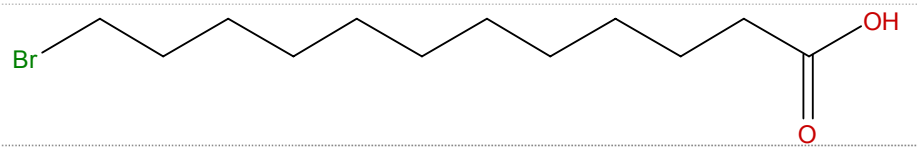

plane group $\quad p 2 g g$ 


\begin{tabular}{ll} 
crystal class & oblique \\
\hline$Z$ & 8 \\
\hline$Z^{\prime}$ & 2 \\
\hline $\begin{array}{l}\text { molecular } \\
\text { symmetry }\end{array}$ & $\mathrm{C}(\mathrm{s})$ \\
site symmetry & 1 \\
\hline $\begin{array}{l}\text { molecular } \\
\text { weight }(\text { Da) }\end{array}$ & 279.21 \\
\hline multicomponent & no
\end{tabular}

Fang, H. B., Giancarlo, L. C. \& Flynn, G. W. J. Phys.

Chem. B 1999 103, 5712-5715.

\section{Record 48}

4-n-decyloxy-4'-cyanobiphenyl (10OCB)

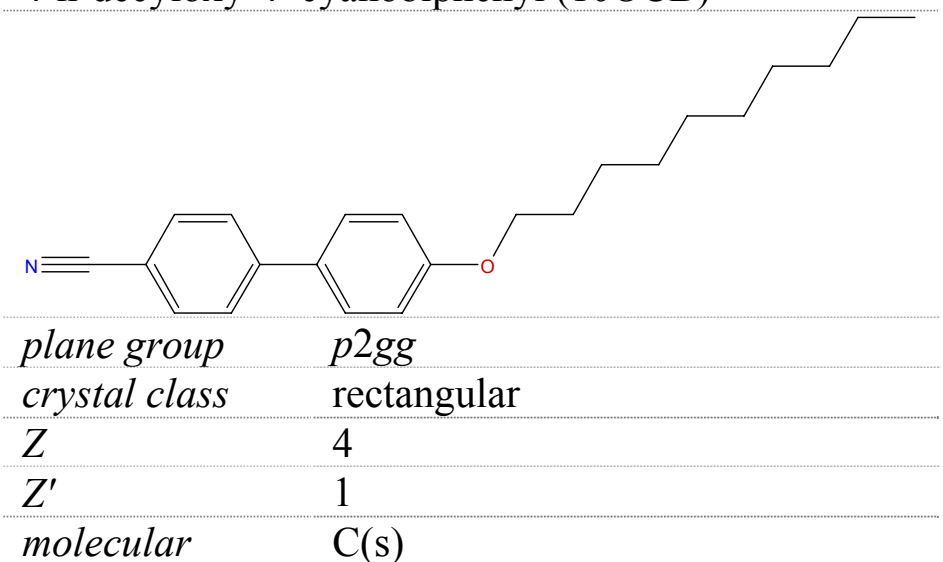

molecular

weight (Da)

multicomponent yes

Mu, Z., Wang, Z., Zhang, X., Ye, K. \& Wang, Y. J.

Phys. Chem. B 2004 108, 19955-19959.

\section{Record 50}

hexatriacontane

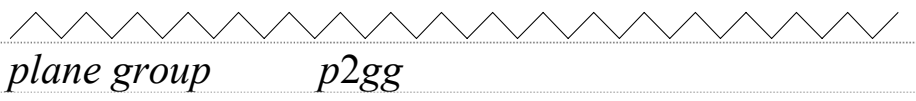

crystal class rectangular

Z 2

$Z^{\prime} \quad 0.5$

molecular $\quad \mathrm{C}(2 \mathrm{~h})$

symmetry

site symmetry 2

molecular $\quad 506.97$

weight (Da)

multicomponent no

Liang, W., Whangbo, M.-H., Wawkuschewski, A., Cantow, H.-J. \& Magonov, S. N. Adv. Mater. 1993 5, 817-821.

\section{Record 51}

5,12-dihexylquinolino[2,3-b]acridine-7,14(5H,12H)dione (QA-C6)

symmetry

site symmetry 1

molecular $\quad 335.48$

weight (Da)

multicomponent no

Taki, S. \& Kai, S. Jpn. J. Appl. Phys., Part 12001 40, 4187-4192.

\section{Record 49}

1,3,8,10-tetramethyl-N,N'-didocosyl-quinacridone TmQA-C22 and steric acid
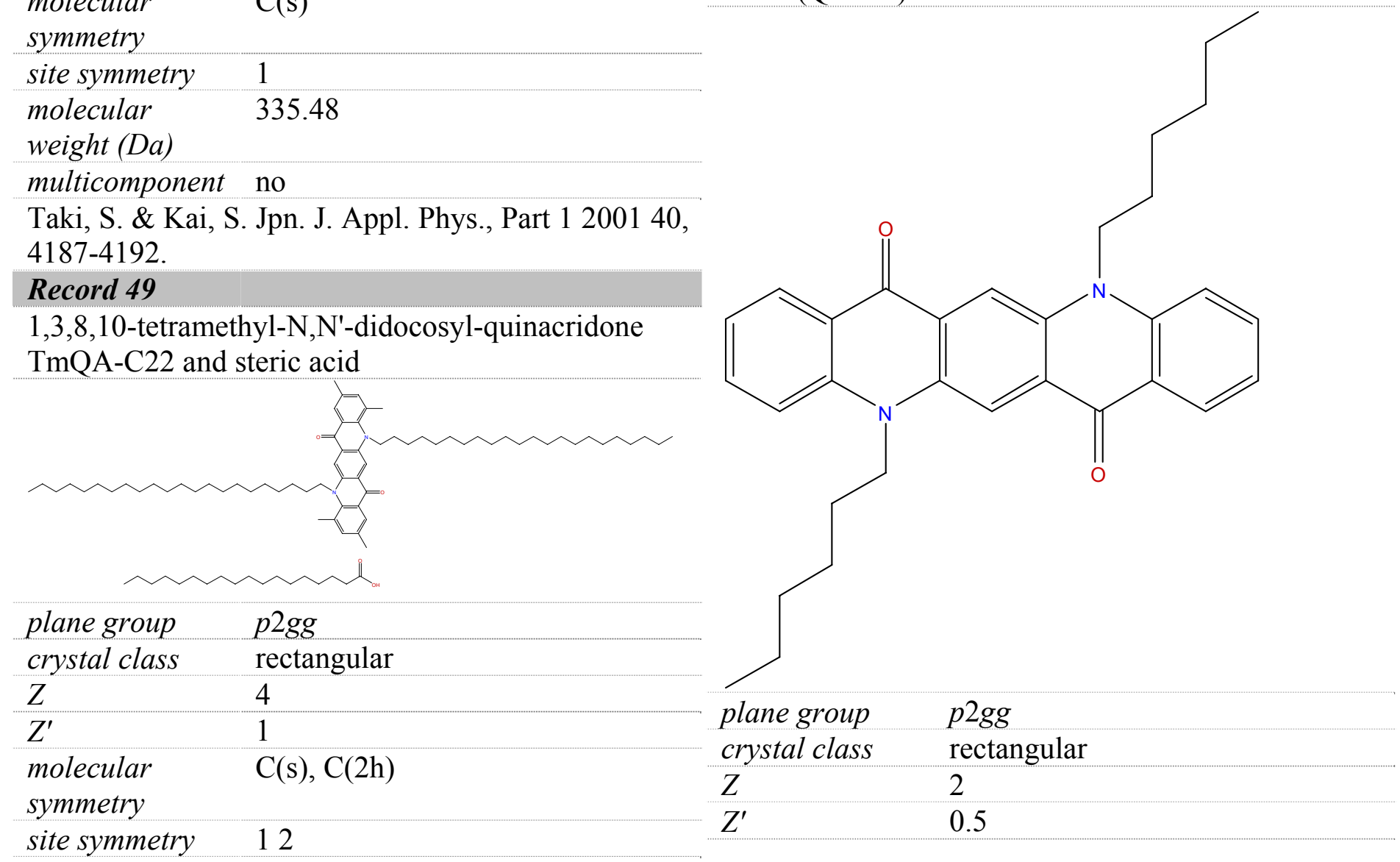


\begin{tabular}{ll}
$\begin{array}{l}\text { molecular } \\
\text { symmetry }\end{array}$ & $\mathrm{C}(2 \mathrm{~h})$ \\
site symmetry & 2 \\
\hline $\begin{array}{l}\text { molecular } \\
\text { weight }(\mathrm{Da})\end{array}$ & 480.64 \\
multicomponent & no \\
Qiu, D., Ye, K., Wang, Y., Zou, B., Zhang, X., Lei, \\
S. \& Wan, L. Langmuir 2003 19, 678-681.
\end{tabular}

S. \& Wan, L. Langmuir 2003 19, 678-681.

\section{Record 52}

3,3"'-dipentyl-2,2':5',2":5",2"':5"',2"'-

quinquethiophene

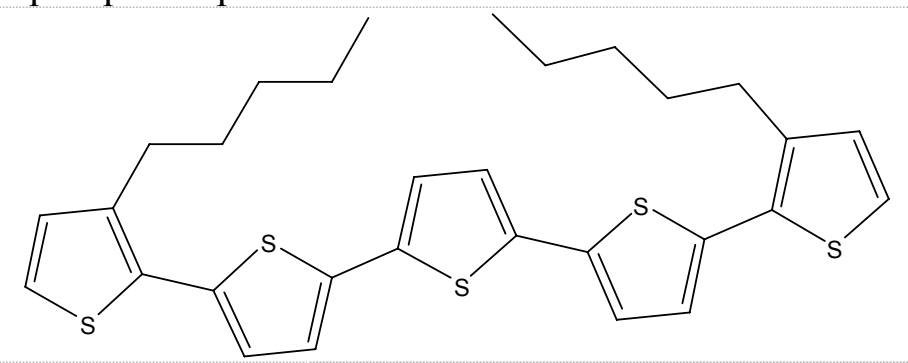

plane group p2gg

crystal class rectangular

$\begin{array}{ll}Z & 4 \\ Z^{\prime} & 1 \\ \text { molecular } & \mathrm{C}(2 \mathrm{v})\end{array}$

symmetry

site symmetry 1

molecular $\quad 552.90$

weight (Da)

multicomponent no

Müller, H., Petersen, J., Strohmaier, R., Gompf, B., Eisenmenger, W., Vollmer, M. S. \& Effenberger, F. Adv. Mater. 1996 8, 733-737.

\section{Record 53}

5-(11,11,12,12,13,13,14,14,15,15,16,16, 17,17,18,18,18-heptadecafluoro-noctadecyloxy)isophthalic acid

F8H10-ISA

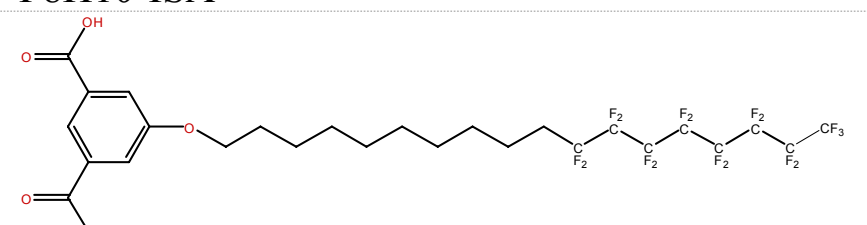

\begin{tabular}{ll} 
plane group & $p 2 g g$ \\
crystal class & rectangular \\
\hline$Z$ & 4 \\
\hline$Z^{\prime}$ & 1 \\
molecular & $\mathrm{C}(\mathrm{s})$ \\
symmetry & \\
site symmetry & 1
\end{tabular}

molecular

740.45

weight (Da)

multicomponent no

Abdel-Mottaleb, M. M. S., De Feyter, S., Sieffert, M., Klapper, M., Müllen, K. \& De Schryver, F. C. Langmuir 2003 19, 8256-8261.

\section{Record 54}

octacosane (in solution with octadecane and decane)

plane group $\quad p 2 g g$

crystal class rectangular

$Z \quad 2$

$Z^{\prime} \quad 0.5$

molecular $\quad \mathrm{C}(2 \mathrm{~h})$

symmetry

site symmetry 2

molecular $\quad 394.76$

weight (Da)

multicomponent yes

Bucher, J.-P., Roeder, H. \& Kern, K. Surf. Sci. 1993 289, 370-380.

\section{Record 55}

didocosyl disulfide

\begin{tabular}{|c|c|}
\hline plane group & $p 2 g g$ \\
\hline crystal class & rectangular \\
\hline$Z$ & 2 \\
\hline$Z^{\prime}$ & 0.5 \\
\hline $\begin{array}{l}\text { molecular } \\
\text { symmetry }\end{array}$ & $C(2 v)$ \\
\hline site symmetry & 2 \\
\hline $\begin{array}{l}\text { molecular } \\
\text { weight }(D a)\end{array}$ & 669.29 \\
\hline \multicolumn{2}{|l|}{ multicompon } \\
\hline \multicolumn{2}{|c|}{$\begin{array}{l}\text { Venkataraman, B., Flynn, G. W., Wilbur, J. L., } \\
\text { Folkers, J. P. \& Whitesides, G. M. J. Phys. Chem. } \\
1995 \text { 99, 8684-8689. }\end{array}$} \\
\hline \multicolumn{2}{|l|}{ Record 56} \\
\hline \multicolumn{2}{|l|}{ triacontane } \\
\hline plane group & $p 2 g g$ \\
\hline crystal class & rectangular \\
\hline$Z$ & 2 \\
\hline$Z^{\prime}$ & 0.5 \\
\hline molecular & $\mathrm{C}(2 \mathrm{~h})$ \\
\hline
\end{tabular}


symmetry

site symmetry 2

molecular

422.81

weight (Da)

multicomponent no

Venkataraman, B., Breen, J. J. \& Flynn, G. W. J.

Phys. Chem. 1995 99, 6608-6619.

\section{Record 57}

15-hydroxypentadecanoic acid

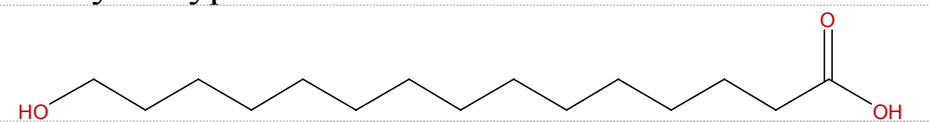

plane group $\quad p 2 g g$

crystal class rectangular

$\begin{array}{ll}Z & 4 \\ Z^{\prime} & 1\end{array}$

molecular $\mathrm{C}(\mathrm{s})$

symmetry

site symmetry 1

molecular $\quad 258.40$

weight (Da)

multicomponent no

Wintgens, D., Yablon, D. G. \& Flynn, G. W. J. Phys. Chem. B 2003 107, 173-179.

\begin{tabular}{|c|c|}
\hline Record 58 & \\
\hline 1,5-bis(3'-thia-te & adecyl)anthracene \\
\hline plane group & $p 2 g g$ \\
\hline crystal class & rectangular \\
\hline$Z$ & 2 \\
\hline$Z^{\prime}$ & 0.5 \\
\hline $\begin{array}{l}\text { molecular } \\
\text { symmetry }\end{array}$ & $C(2 h)$ \\
\hline site symmetry & 2 \\
\hline $\begin{array}{l}\text { molecular } \\
\text { weight }(D a)\end{array}$ & 607.05 \\
\hline multicomponent & no \\
\hline $\begin{array}{l}\text { Wei, Y., Kannap } \\
\text { B. J. Am. Chem. }\end{array}$ & $\begin{array}{l}\text { an, K., Flynn, G. W. \& Zimmt, M. } \\
\text { Soc. } 2004 \text { 126, 5318-5322. }\end{array}$ \\
\hline
\end{tabular}

\section{Record 59}

nonadecanoic acid

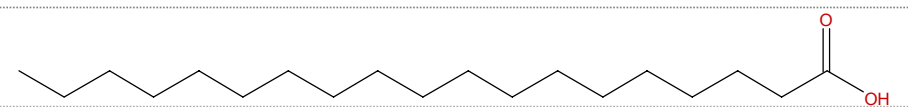

plane group $\quad p 2 g g$

crystal class rectangular

$Z \quad 4$

$Z^{\prime} \quad 1$

molecular $\mathrm{C}(\mathrm{s})$

symmetry

site symmetry 1

molecular $\quad 298.50$

weight (Da)

multicomponent no

Hibino, M., Sumi, A., Tsuchiya, H. \& Hatta, I. J.

Phys. Chem. B 1998 102, 4544-4547.

\section{Record 60}

perylene-3,4-9-10-tetracarboxylic-dianhydride

(PTCDA)

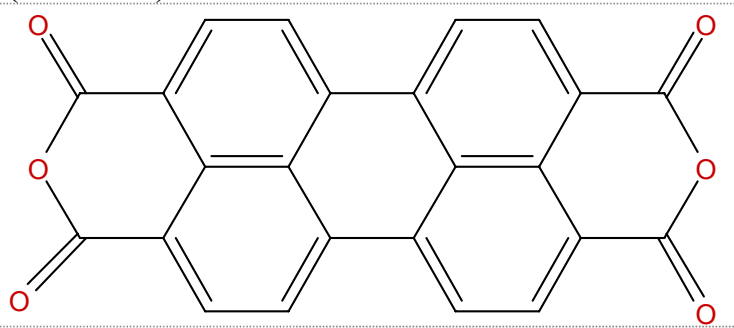

plane group p2gg

crystal class rectangular

$Z \quad 2$

$Z^{\prime} \quad 0.5$

molecular $\mathrm{D}(2 \mathrm{~h})$

symmetry

site symmetry 2

molecular $\quad 392.32$

weight (Da)

multicomponent no

Takajo, D., Nemoto, T., Kurata, H., Isoda, S., Ozaki, H. \& Mazaki, Y. Thin Solid Films 2003 438, 428432.

\section{Record 61}

cyclooctatetracontane $(\mathrm{CH} 2) 48$ 


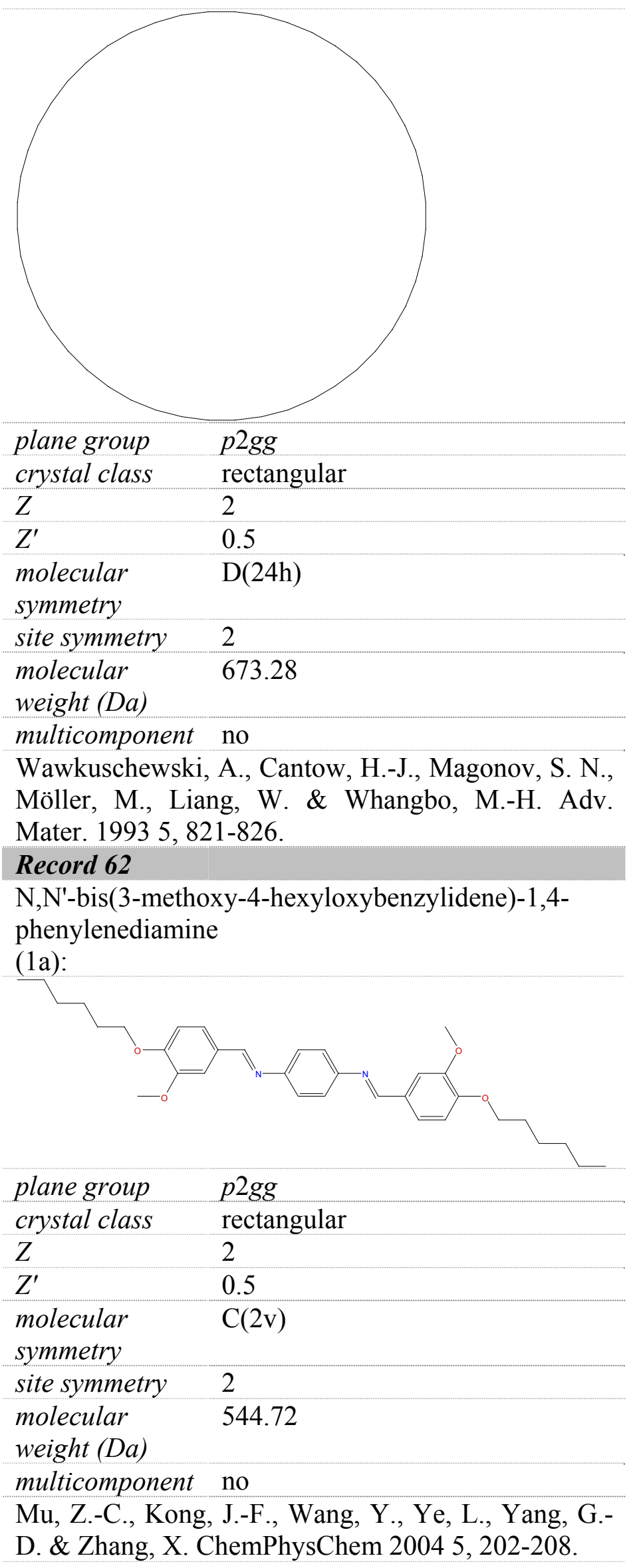

\section{Record 63}

1,12-dodecanediol

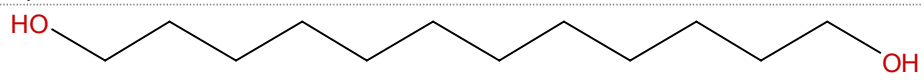

\begin{tabular}{|c|c|}
\hline plane group & $p 2 g g$ \\
\hline crystal class & rectangular \\
\hline$Z$ & 2 \\
\hline$Z^{\prime}$ & 0.5 \\
\hline $\begin{array}{l}\text { molecular } \\
\text { symmetry }\end{array}$ & $\mathrm{C}(2 \mathrm{~h})$ \\
\hline site symmetry & 2 \\
\hline $\begin{array}{l}\text { molecular } \\
\text { weight }(D a)\end{array}$ & 202.33 \\
\hline multicomponent & 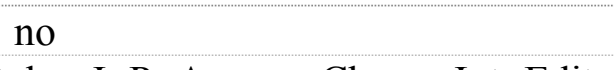 \\
\hline
\end{tabular}

\section{Record 64}

bis(4-dibutylamino-2-phenyl)squaraine

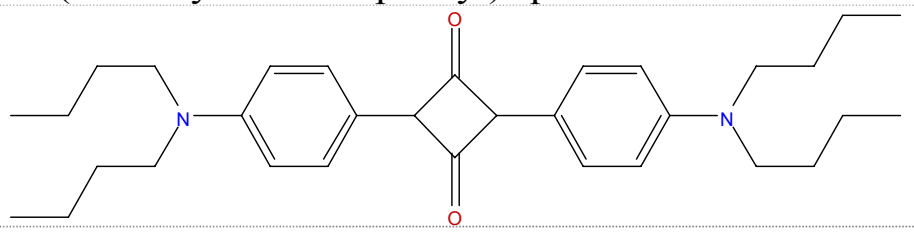

plane group $\quad p 2 g g$

crystal class oblique

Z 2

$Z^{\prime} \quad 0.5$

molecular $\quad \mathrm{C}(2 \mathrm{v})$

symmetry

site symmetry 2

molecular $\quad 490.72$

weight ( $D a)$

multicomponent no

Yasuda, S. \& Shigekawa, H. Jpn. J. Appl. Phys., Part

12003 42, 4901-4904.

\section{Record 65}

7-[2-(3-Dodecylcarbamoyl-propoxy)-phenoxy]-

heptanoic acid dodecylamide (3)

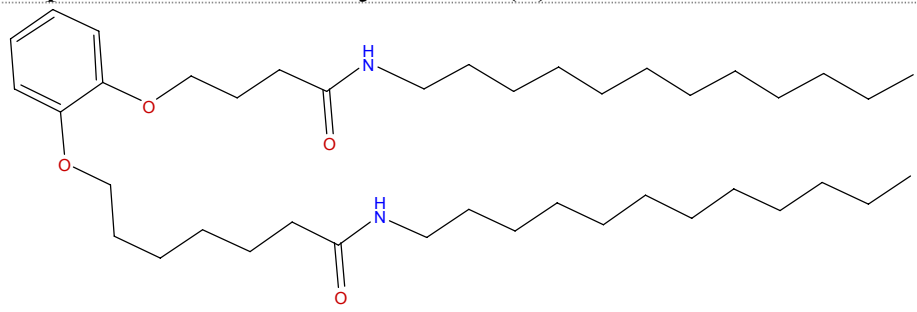

plane group $\quad p 2 g g$

crystal class rectangular 


$\begin{array}{ll}Z & 4 \\ Z \begin{array}{l}Z \\ Z\end{array} & 1 \\ \text { molecular } & \mathrm{C}(\mathrm{s}) \\ \text { symmetry } & \\ \text { site symmetry } & 1 \\ \text { molecular } & 659.04 \\ \text { weight (Da) } \\ \text { multicomponent no } \\ \text { Schuurmans, N., Uji-i, H., Mamdouh, W., De } \\ \text { Schryver, F. C., Feringa, B. L., Van Esch, J. \& De } \\ \text { Feyter, S. J. Am. Chem. Soc. 2004 126, 13884- } \\ \text { 13885. }\end{array}$

\section{Record 66}

2-(2-hydroxyethoxy)ethyl $3,4,5-\operatorname{tris}(4-$ (dodecyloxy)benzyloxy)benzoate

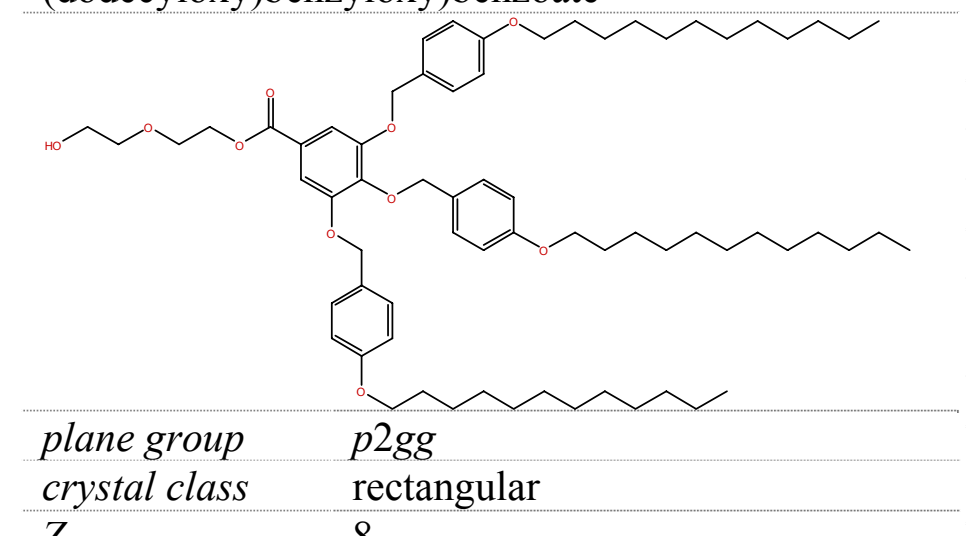

\begin{tabular}{ll}
$Z$ & 8 \\
$Z^{\prime}$ & 2 \\
\hline
\end{tabular}

molecular $\mathrm{C}(\mathrm{s})$

symmetry

site symmetry 11

molecular $\quad 1081.55$

weight (Da)

multicomponent no

Mamdouh, W., Uji-i, H., Dulcey, A. E., Percec, V., De Feyter, S. \& De Schryver, F. C. Langmuir 2004 20, 7678-7685.

\section{Record 67}

2,5,8,11,14-pentadodecyl-17-undecylamine-hexaperi-hexa-benzocoronene $2 \mathrm{a}(15 \mathrm{a})$

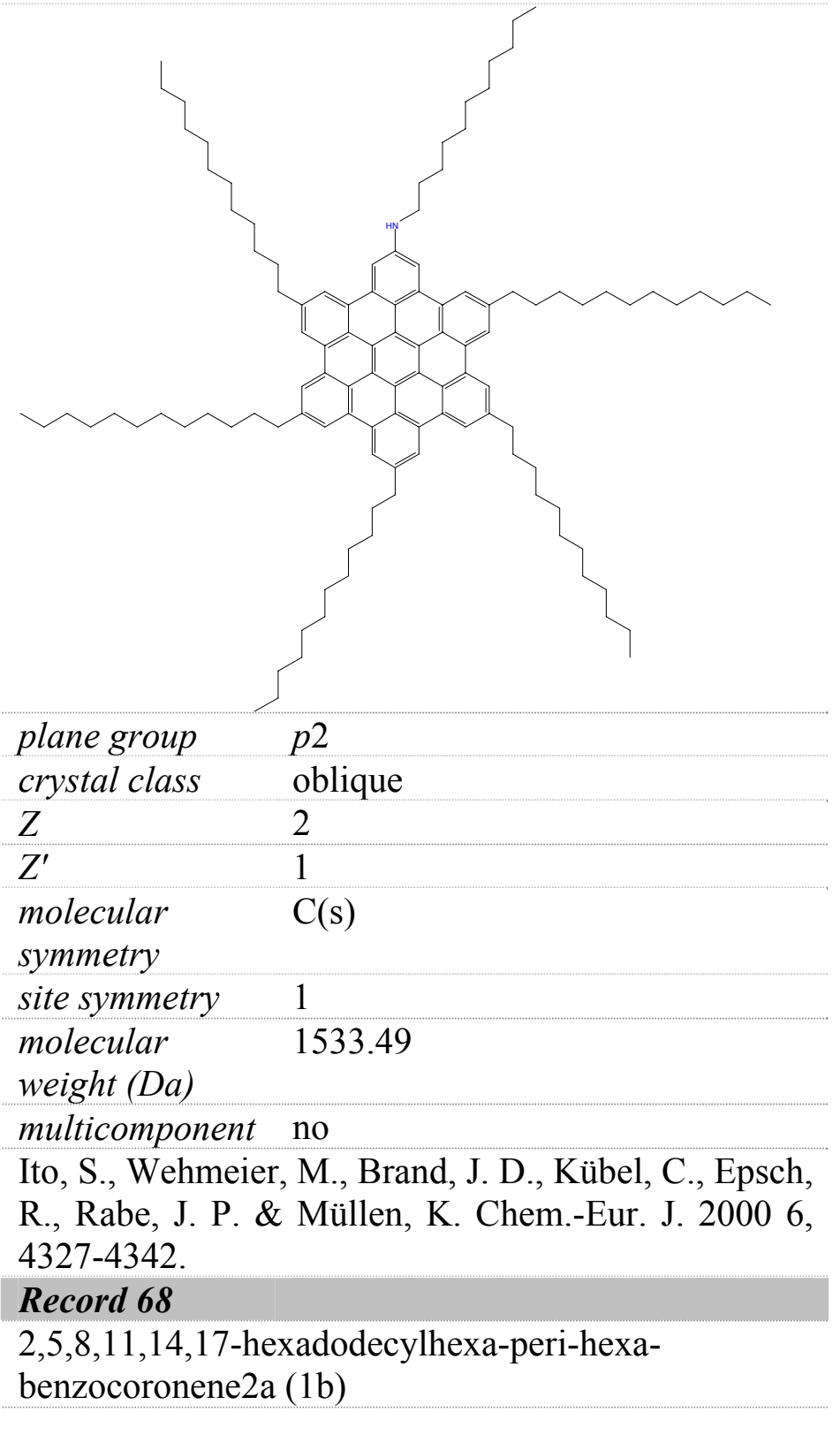

benzocoronene $2 \mathrm{a}(1 \mathrm{~b})$

\section{Record 68}

\section{plane group p}

$Z \quad 2$

$Z^{\prime}$

symolecular

site symmetry 1

molecular $\quad 1533.49$

wh (Da)

Ito, S., Wehmeier, M., Brand, J. D., Kübel, C., Epsch, R., Rabe, J. P. \& Müllen, K. Chem.-Eur. J. 2000 6, 4327-4342.

.




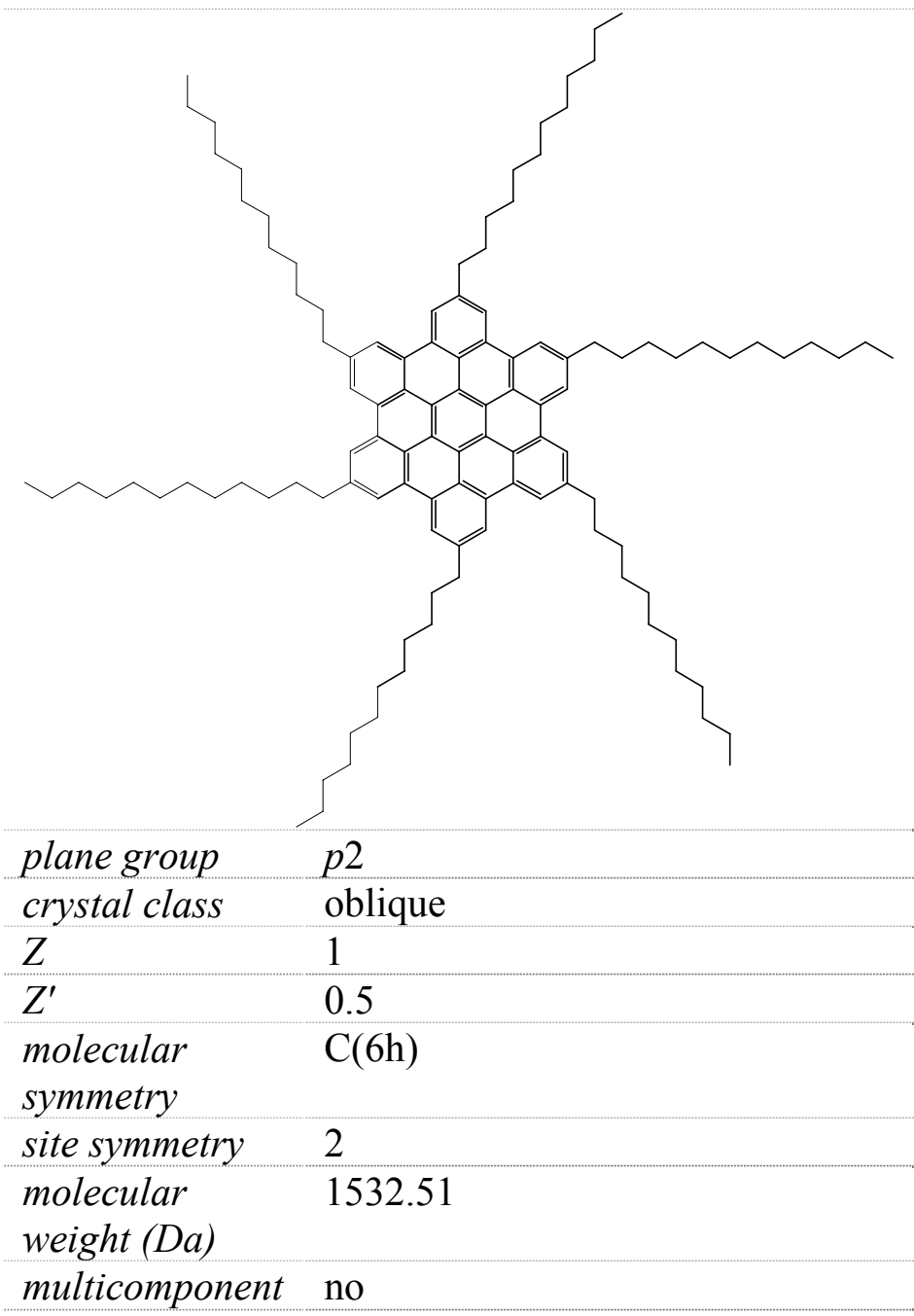

Ito, S., Wehmeier, M., Brand, J. D., Kübel, C., Epsch, R., Rabe, J. P. \& Müllen, K. Chem.-Eur. J. 2000 6, 4327-4342.

\section{Record 69}

2,5,8,11,14-pentadodecylhexa-peri-hexabenzocoronene2a (1d)

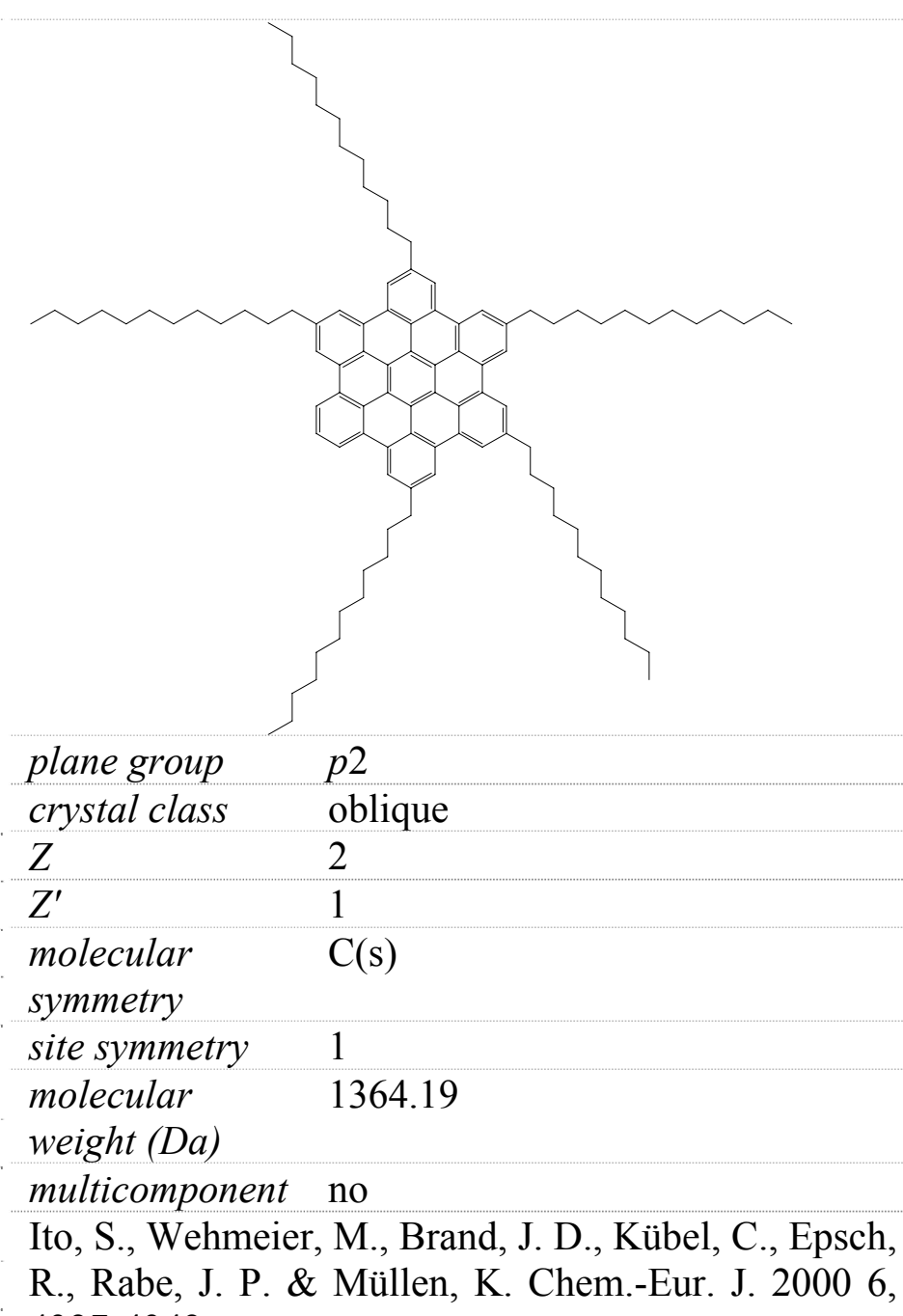

4327-4342.

\section{Record 70}

dihexylsexithiophene (T6H2)

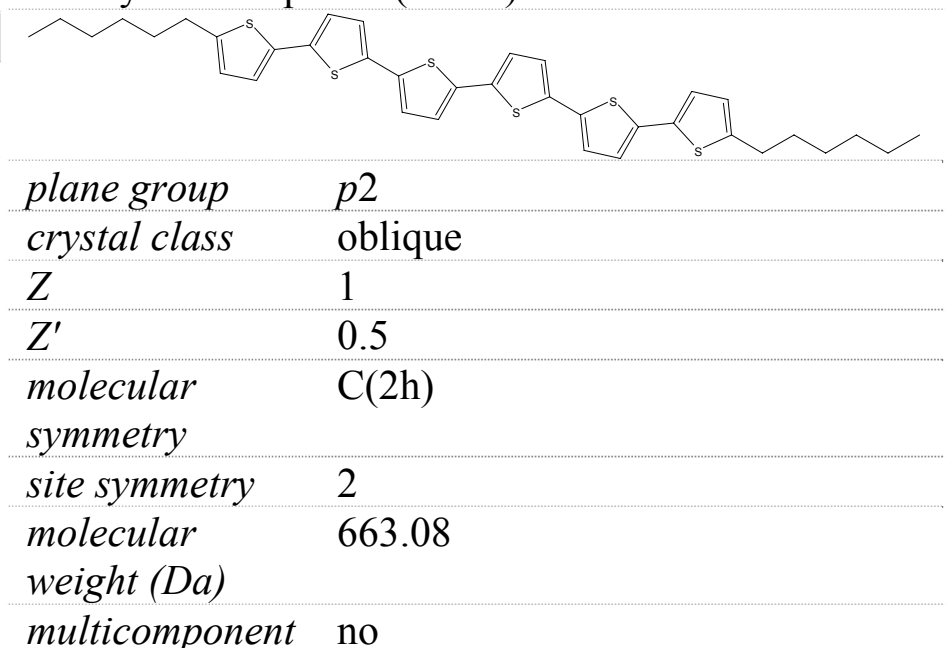

Stabel, A. \& Rabe, J. P. Synth. Met. 1994 67, 47-53.

\section{Record 71}

3,4,5-tris[(dodecyloxy)-p-benzyloxy]benzoic acid (5). 


\begin{tabular}{|c|c|}
\hline & $\begin{array}{l}\text { molecular } \quad \mathrm{C}(\mathrm{s}) \\
\text { symmetry }\end{array}$ \\
\hline & site symmetry \\
\hline & $\begin{array}{l}\text { molecular } \\
\text { weight }(D a)\end{array}$ \\
\hline & $\begin{array}{l}\text { multicomponent no } \\
\text { Hibino, M., Sumi, A. \& Hatta, I. Jpn. J. Appl. Phys., } \\
\text { Part } 1 \text { 1995 34, 3354-3359. }\end{array}$ \\
\hline nlane oroun $\quad n$ 2 & Record 74 \\
\hline $\begin{array}{ll}\text { plane group } & p 2 \\
\text { crystal class } & \text { oblique }\end{array}$ & 4-n-octyl-4'-cyanobiphenyl (8CB) \\
\hline$Z$ & \\
\hline$Z^{\prime}$ & \\
\hline $\begin{array}{l}\text { molecular } \\
\text { symmetry }\end{array}$ & \\
\hline site symmetry 1 & \\
\hline $\begin{array}{l}\text { molecular } \\
\text { weight }(\mathrm{Da})\end{array} \quad 993.44$ & plane group \\
\hline multicomponent no & $\begin{array}{ll}\text { crystal class } & \text { oblique } \\
Z & 2\end{array}$ \\
\hline Prokhorova, S. A., Sheiko, S. S., Mourran, A., & $Z^{\prime}$ \\
\hline $\begin{array}{l}\text { Azum1, R., Beginn, U., Zipp, G., Ahn, C.-H., } \\
\text { Holerca, M. N., Percec, V. \& Möller, M. Langmuir } \\
2000 \text { 16, 6862-6867. }\end{array}$ & $\begin{array}{l}\text { molecular } \quad \mathrm{C}(\mathrm{s}) \\
\text { symmetry }\end{array}$ \\
\hline Record 72 & site symmetry 1 \\
\hline $\mathrm{N}, \mathrm{N}$-dodecanomethylenebishexanoamide. & $\begin{array}{l}\text { molecular } \quad 291.43 \\
\text { weight }(\mathrm{Da})\end{array}$ \\
\hline plane group $\quad p 2$ & $\begin{array}{l}\text { multicomponent no } \\
\text { Patrick, D. L. \& Beebe, T. P. Langmuir } 1994 \text { 10, } \\
\text { 298-302. }\end{array}$ \\
\hline crystal class oblique & Record 75 \\
\hline$Z$ & 4-n-dodecyl-4'-cyanobiphenyl (12CB) \\
\hline$Z^{\prime}$ & \\
\hline $\begin{array}{l}\text { molecular } \\
\text { symmetry }\end{array}$ & \\
\hline site symmetry & \\
\hline $\begin{array}{l}\text { molecular } \\
\text { weight }(\mathrm{Da})\end{array}$ & \\
\hline multicomponent no & plane group \\
\hline $\begin{array}{l}\text { L1m, R., L1, J., L1, S. F. Y., Feng, Z. \& Valiyaveett1l, } \\
\text { S. Langmuir } 2000 \text { 16, 7023-7030. }\end{array}$ & crystal class \\
\hline Record 73 & 10 \\
\hline myristic acid & $Z^{\prime}$ \\
\hline & $\begin{array}{l}\text { molecular } \\
\text { symmetry }\end{array}$ \\
\hline & site symmetry \\
\hline plane group & molecular \\
\hline crystal class & weight $(D a)$ \\
\hline$Z$ & multicomponent no \\
\hline$Z^{\prime}$ & \\
\hline
\end{tabular}


Freund, J., Probst, O., Grafström, S., Dey, S., Kowalski, J., Neumann, R., Wörtge, M. \& zu Putlitz, G. J. Vac. Sci. Technol., B 1994 12, 1914-1917.

\section{Record 76}

S-2-eicosyloxy-5-(1-

methylheptyloxy)terephthalic acid

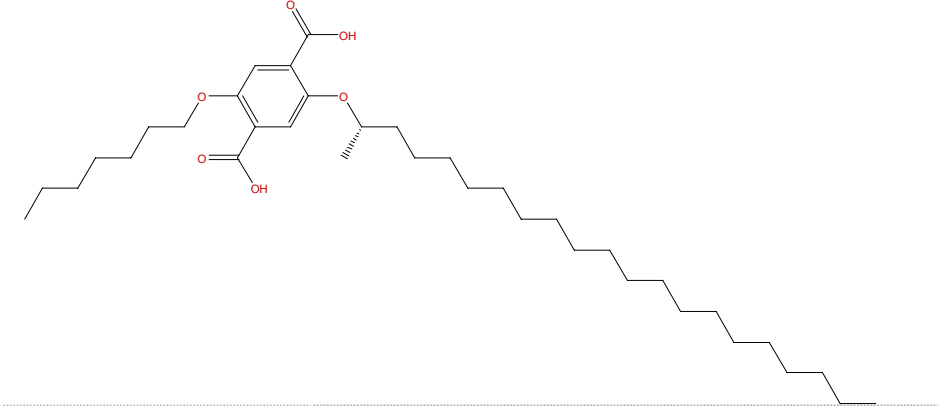

$\begin{array}{ll}\text { plane group } & p 2 \\ \text { crystal class } & \text { oblique } \\ Z & 2\end{array}$

$Z^{\prime}$

molecular $\mathrm{C}(1)$

symmetry

site symmetry 1

molecular $\quad 590.87$

weight (Da)

multicomponent no

De Feyter, S., Gesquière, A., De Schryver, F., Meiners, C., Sieffert, M. \& Müllen, K. Langmuir 2000 16, 9887-9894.

\section{Record 77}

arachidic acid

\begin{tabular}{|c|c|}
\hline plane group & $p 2$ \\
\hline crystal class & oblique \\
\hline$Z$ & 2 \\
\hline$Z^{\prime}$ & 1 \\
\hline $\begin{array}{l}\text { molecular } \\
\text { symmetry }\end{array}$ & $\mathrm{C}(\mathrm{s})$ \\
\hline site symmetry & 1 \\
\hline $\begin{array}{l}\text { molecular } \\
\text { weight }(D a)\end{array}$ & 312.53 \\
\hline \multicolumn{2}{|l|}{ multicomponent } \\
\hline \multicolumn{2}{|c|}{$\begin{array}{l}\text { Hatta, I., Nishino, J., Sumi, A. \& Hibino, M. Jpn. J. } \\
\text { Appl. Phys., Part } 11995 \text { 34, 3930-3936. }\end{array}$} \\
\hline \multicolumn{2}{|c|}{ Record 78} \\
\hline octadecyl 2,3,5-tı & iiodobenzoate \\
\hline
\end{tabular}

\section{Record 78}

octadecyl 2,3,5-triiodobenzoate

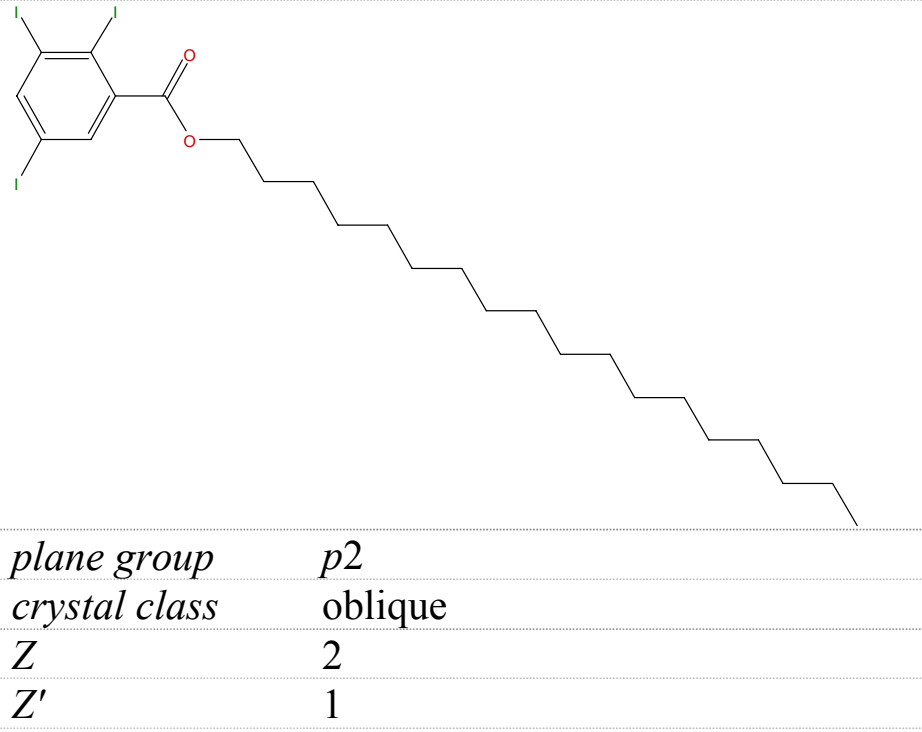

molecular $\quad \mathrm{C}(1)$

symmetry

site symmetry 1

molecular $\quad 752.29$

weight (Da)

multicomponent no

Rabe, J. P. \& Buchholz, S. Makromol. Chem., Macromol. Symp. 1991 50, 261-268.

\section{Record 79}

5-(hexadecyloxy)benzene-1,3-dioic acid (C16ISA)

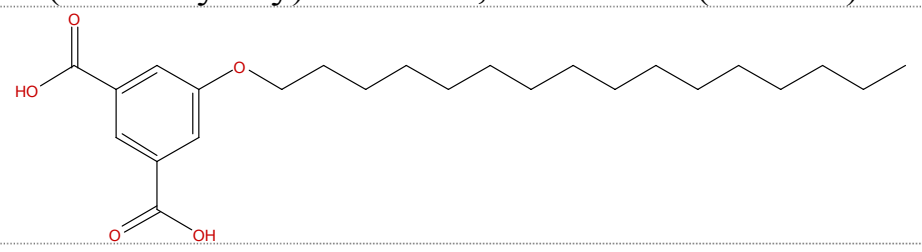

plane group $\quad p 2$

crystal class oblique

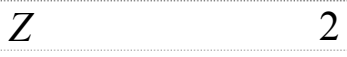

$Z^{\prime} \quad 1$

molecular $\quad \mathrm{C}(\mathrm{s})$

symmetry

site symmetry 1

molecular $\quad 406.56$

weight (Da)

multicomponent no

Eichhorst-Gerner, K., Stabel, A., Moessner, G., Declerq, D., Valiyaveettil, S., Enkelmann, V., Müllen, K. \& Rabe, J. P. Angew. Chem., Int. Edit. 1996 35, 1492-1495.

\section{Record 80}

2,2'-Bis(5,8,11,14,17-penta-n-dodecylhexa-perihexabenzocoronenyl)

(3a). 


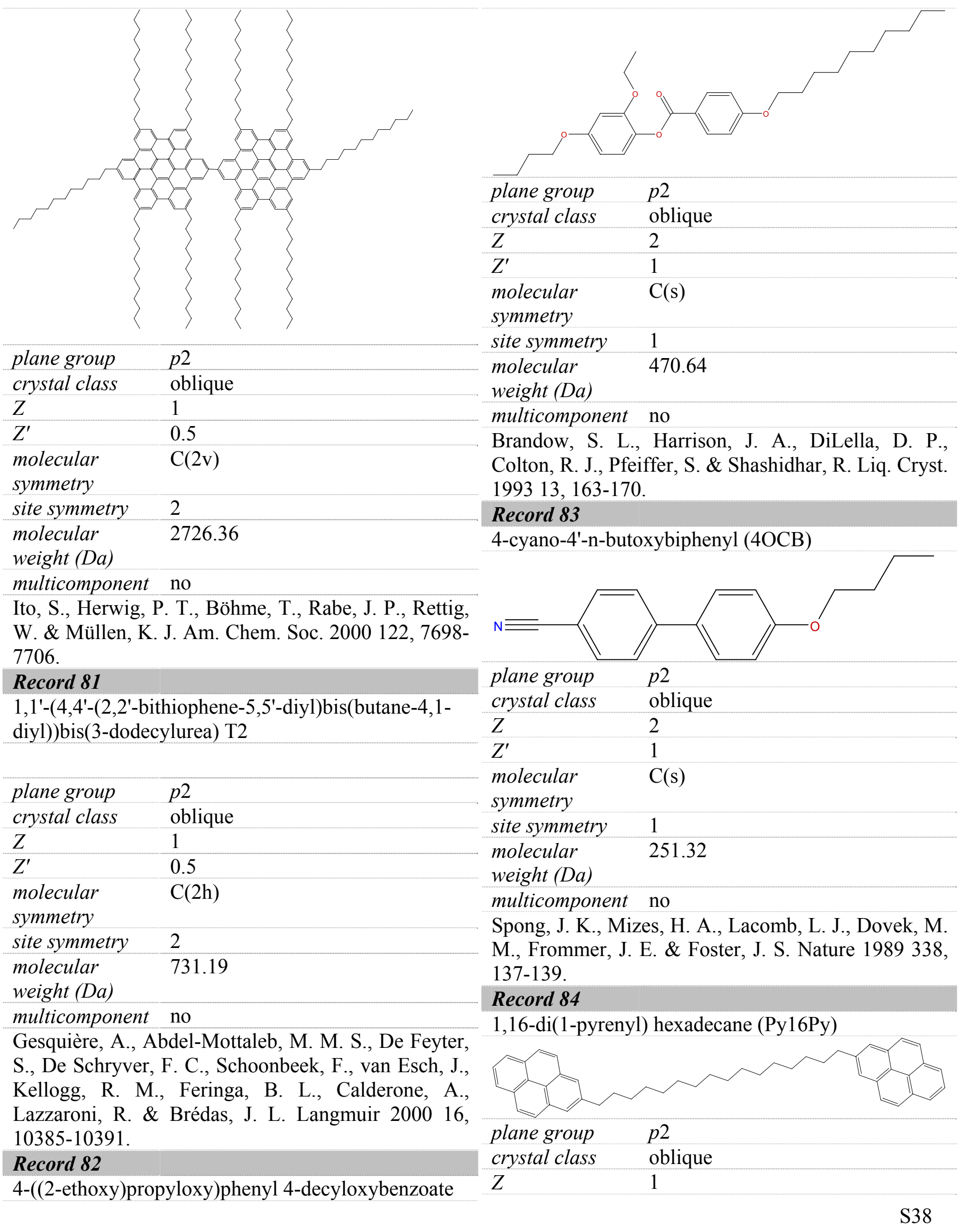


$Z^{\prime}$

molecular

symmetry

site symmetry 2

molecular 626.91

weight (Da)

multicomponent no

Nishio, S., Yoshidome, M., Uji-i, H., Hobley, J., Fukumura, H. \& Zachariasse, K. A. Chem. Lett. 2004, 33, 1506-1507.

\section{Record 85}

4'-dodecyl-4-cyanobiphenyl (12CB)

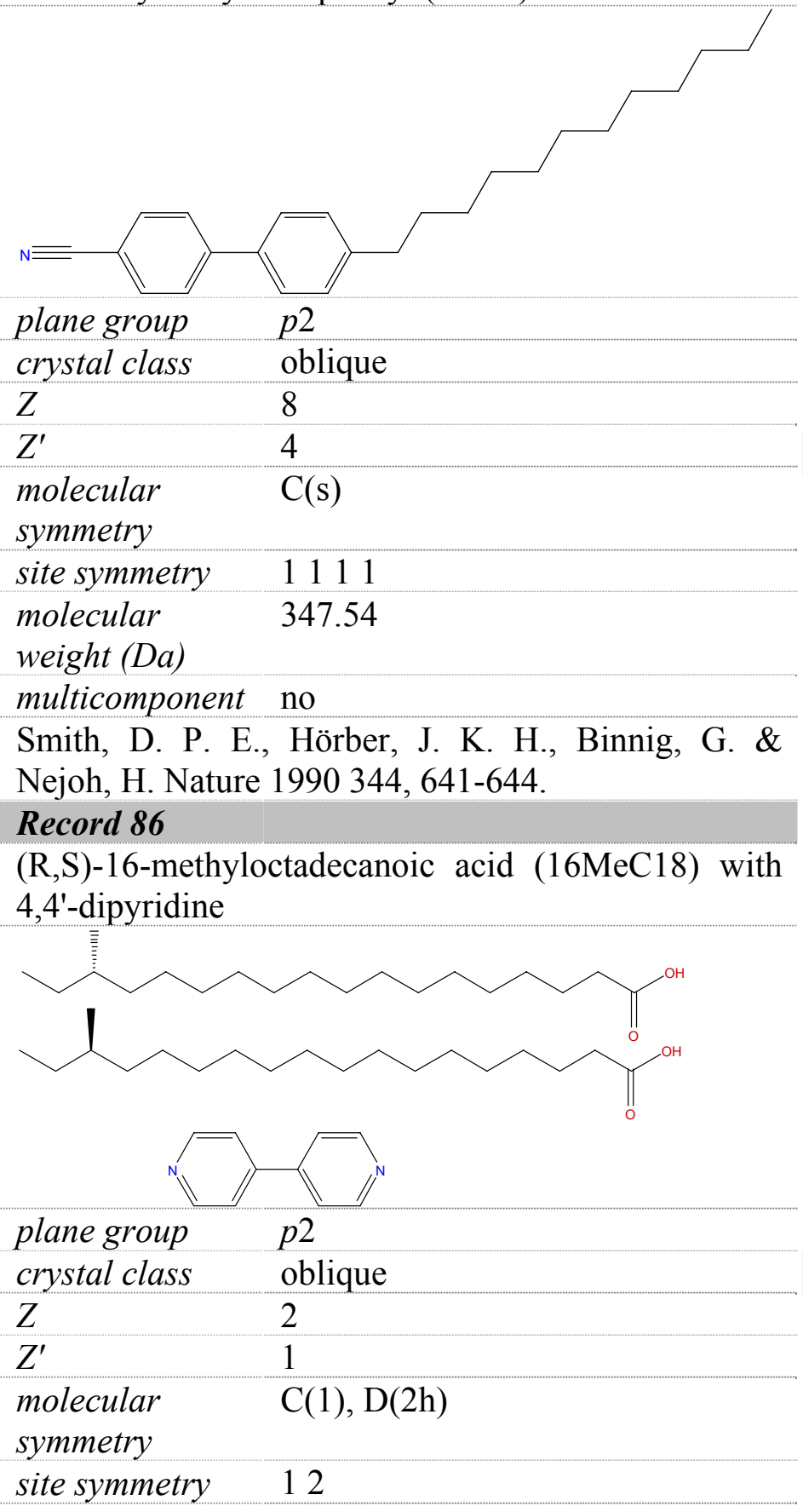

molecular

753.19

weight (Da)

multicomponent yes

Qian, P., Nanjo, H., Sanada, N., Yokoyama, T. \& Suzuki, T. M. Chem. Lett. 2000 1118-1119.

\section{Record 87}

N-octadecylsalicylaldimine (C18Sal)

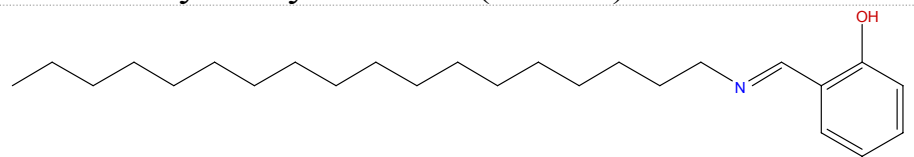

plane group $\quad p 2$

crystal class oblique

$Z \quad 2$

$Z^{\prime} \quad 1$

molecular $\quad \mathrm{C}(\mathrm{s})$

symmetry

site symmetry 1

molecular $\quad 373.62$

weight (Da)

multicomponent no

Qian, P., Nanjo, H., Sanada, N., Yokoyama, T. \& Suzuki, T. M. Chem. Lett. 2000 1118-1119.

\section{Record 88}

1,4-didodecylbenzene (DDB)

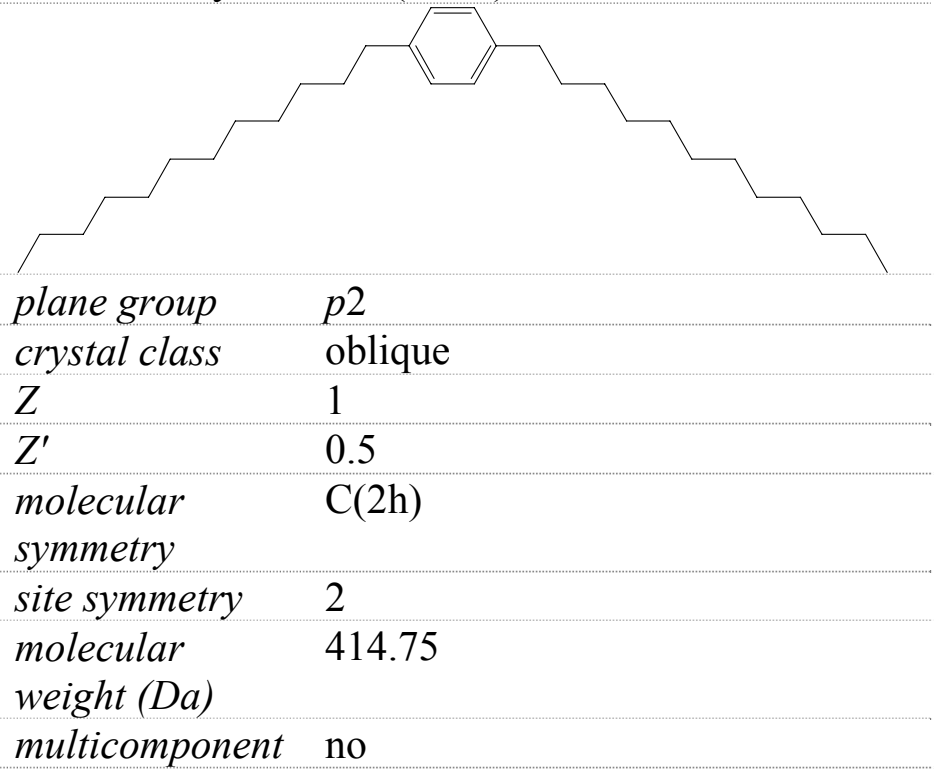

Buchholz, S. \& Rabe, J. P. J. Vac. Sci. Technol., B 1991 9, 1126-1128.

\section{Record 89}

$\mathrm{Cu}(\mathrm{C} 18 \mathrm{Sal}) 2$ 


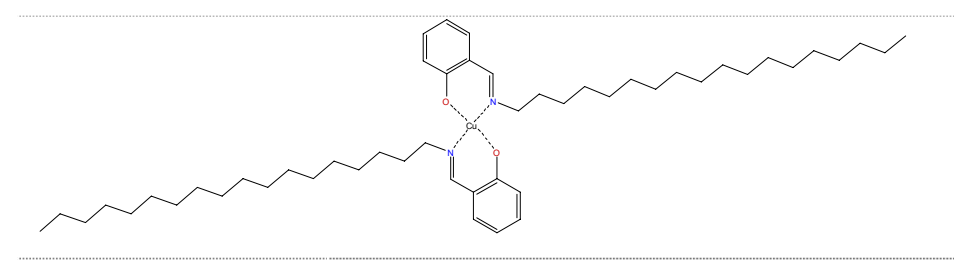

plane group p2

crystal class oblique

$\begin{array}{ll}Z & 1 \\ Z^{\prime} & 0.5\end{array}$

molecular $\mathrm{C}(2 \mathrm{~h})$

symmetry

site symmetry 2

molecular $\quad 808.76$

weight (Da)

multicomponent yes

Qian, P., Nanjo, H., Sanada, N., Yokoyama, T. \& Suzuki, T. M. Chem. Lett. 2000 1118-1119.

\section{Record 90}

5,5-dibromo-3,3-dipentyl-2,2':5',2:5,2'":5"',2-

quinquethiophene $(5 \mathrm{~b})$

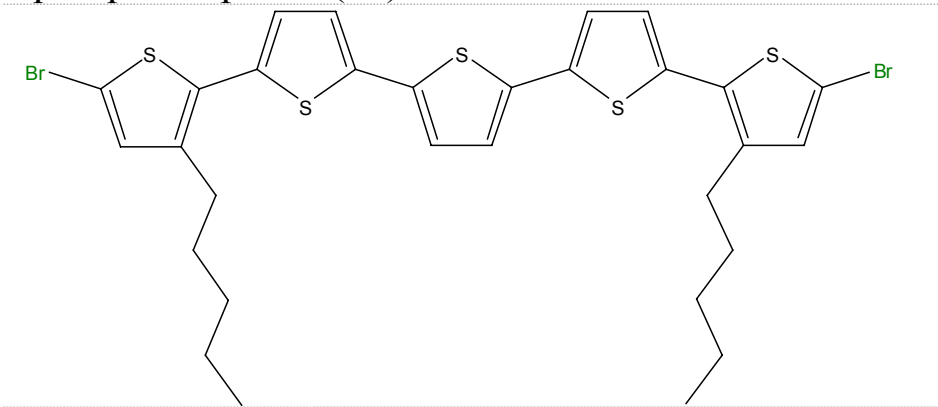

plane group p2

crystal class oblique

$\begin{array}{ll}Z & 2 \\ Z^{\prime} & 1\end{array}$

molecular $\quad \mathrm{C}(2 \mathrm{v})$

symmetry

site symmetry 1

molecular $\quad 710.69$

weight (Da)

multicomponent no

Stecher, R., Gompf, B., Münter, J. S. R. \& Effenberger, F. Adv. Mater. 1999 11, 927-931.

\section{Record 91}

4-n-hexyl-4'-cyanobiphenyl (6CB)

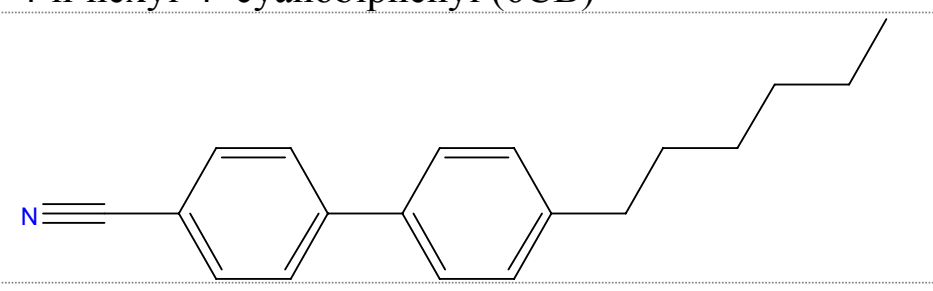

plane group $\quad$ p2

crystal class oblique

$Z \quad 4$

$Z^{\prime} \quad 2$

molecular $\mathrm{C}(\mathrm{s})$

symmetry

site symmetry 11

molecular $\quad 263.38$

weight (Da)

multicomponent no

Mizutani, W., Shigeno, M., Ohmi, M., Suginoya, M., Kajimura, K. \& Ono, M. J. Vac. Sci. Technol., B 1991 9, 1102-1106.

\section{Record 92}

2,5-didodecylsexithiophene (2,5-T6D2)

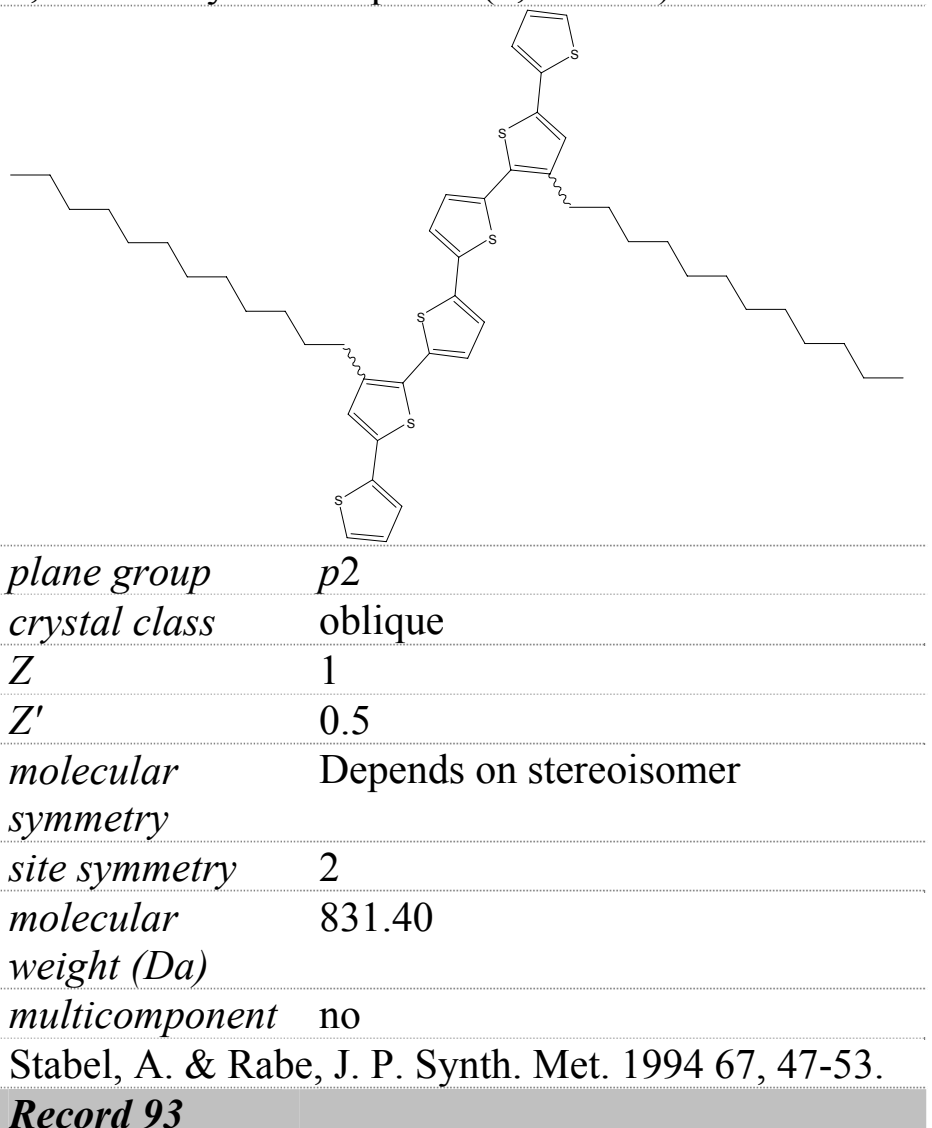

3,3-Dipentyl-2,2':5',2:5,2"':5"',2-quinquethiophene-5carboxylic (4a) 


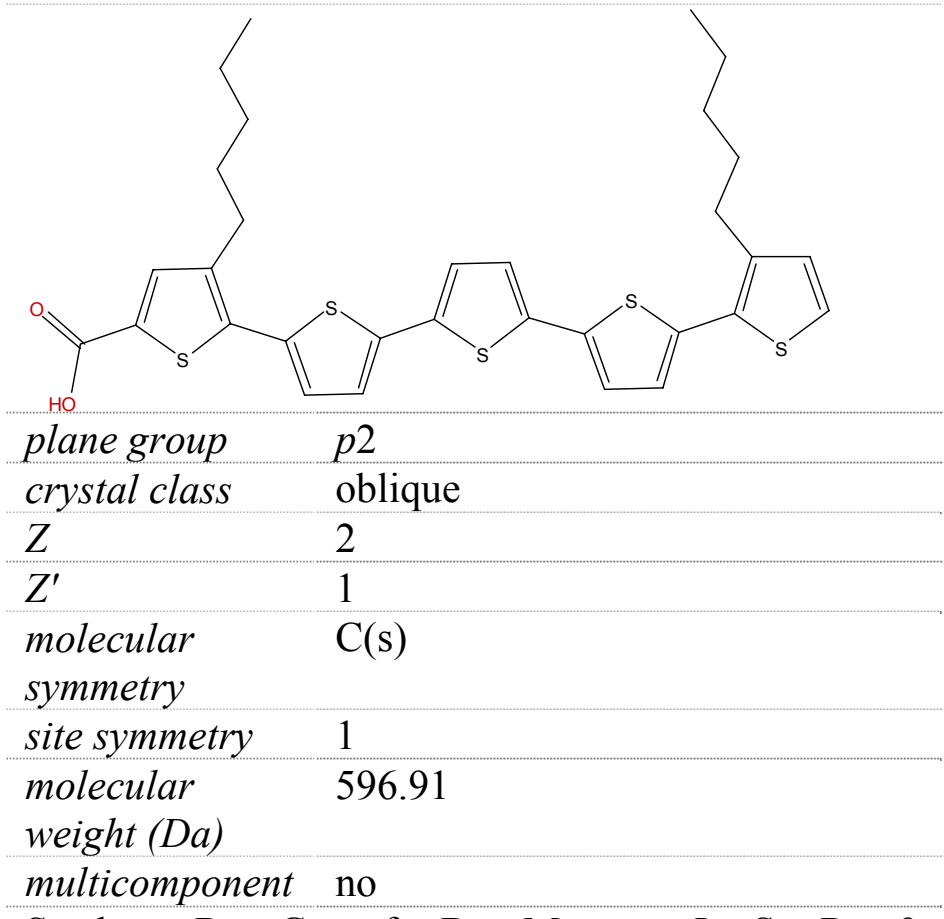

Stecher, R., Gompf, B., Münter, J. S. R. \& Effenberger, F. Adv. Mater. 1999 11, 927-931.

\section{Record 94}

2,5-didodecylsexithiophene (regioselective 2,5T6D2)

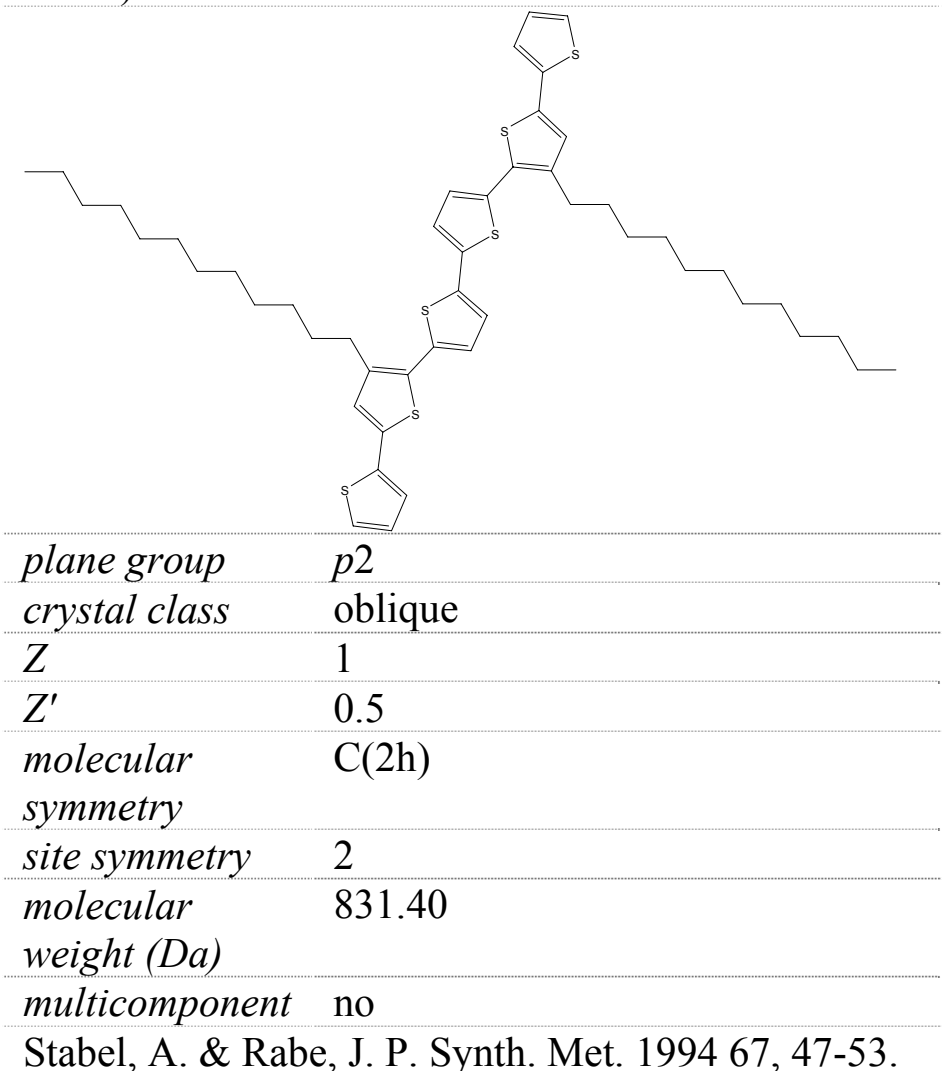

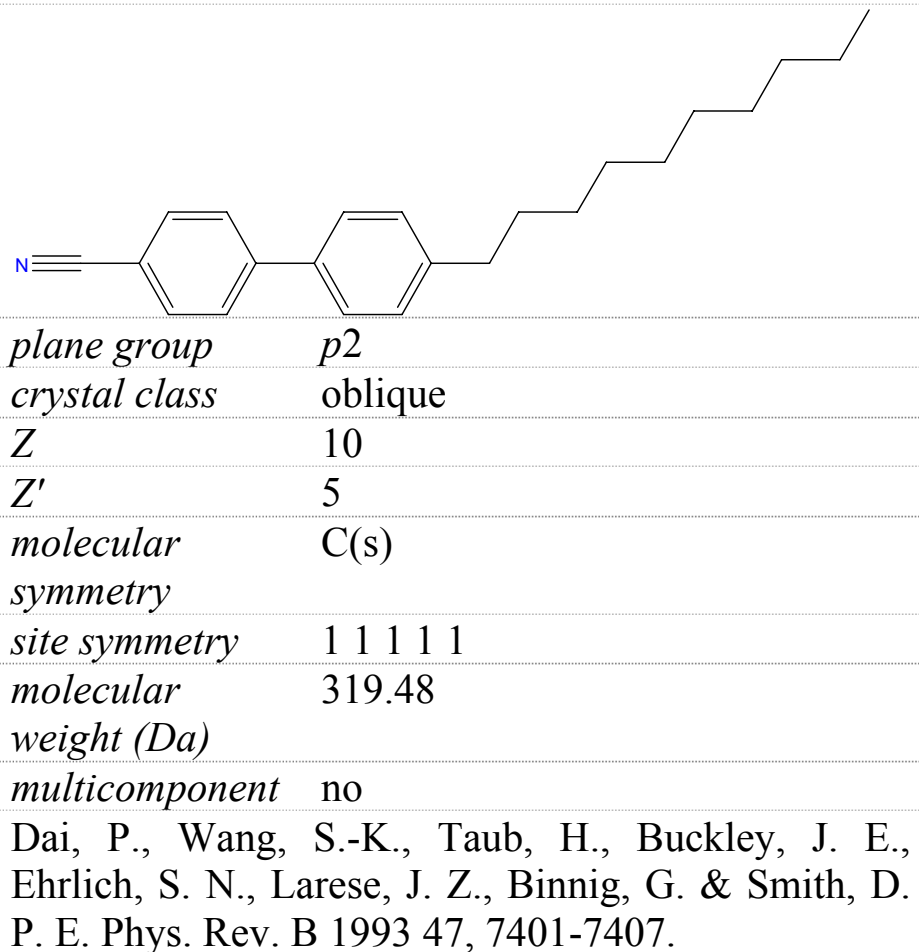

Record 96

4-n-decyl-4'-cyanobiphenyl (10CB)

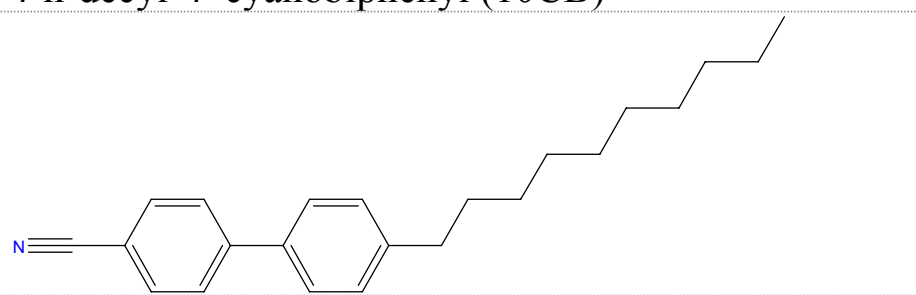

plane group $\quad p 2$

crystal class oblique

$Z \quad 6$

$Z^{\prime} \quad 3$

molecular $\quad \mathrm{C}(\mathrm{s})$

symmetry

site symmetry $\quad 111$

molecular $\quad 319.48$

weight $(D a)$

multicomponent no

Dai, P., Wang, S.-K., Taub, H., Buckley, J. E., Ehrlich, S. N., Larese, J. Z., Binnig, G. \& Smith, D. P. E. Phys. Rev. B 1993 47, 7401-7407.

\section{Record 97}

4-((S)-2-ethoxypropoxy)phenyl

$4-$

\section{Record 95}

4-n-decyl-4'-cyanobiphenyl (10CB) 


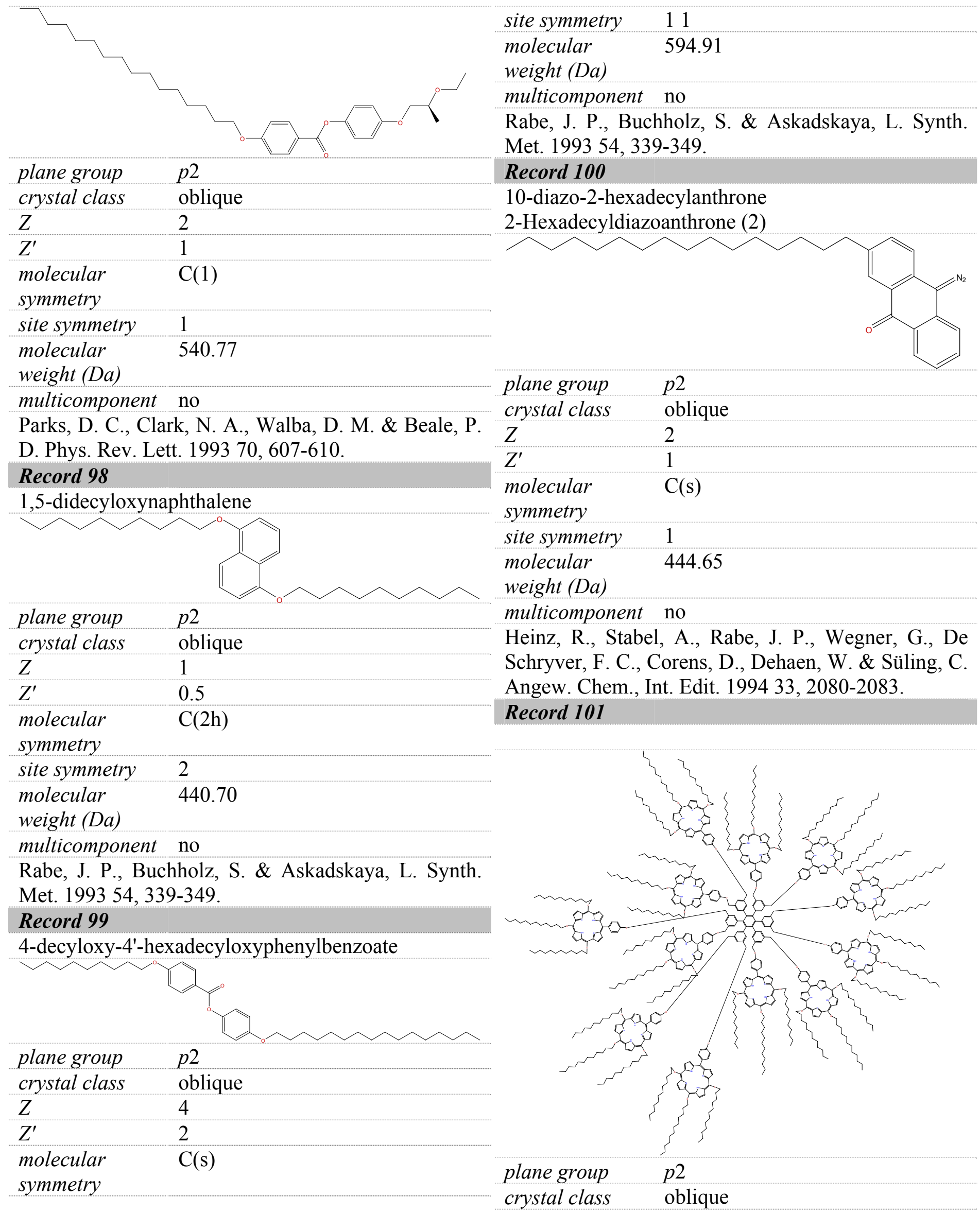




\begin{tabular}{|c|c|}
\hline$Z$ & 1 \\
\hline$Z^{\prime}$ & 0.5 \\
\hline $\begin{array}{l}\text { molecular } \\
\text { symmetry }\end{array}$ & $\mathrm{D}(6 \mathrm{~h})$ \\
\hline site symmetry & 2 \\
\hline $\begin{array}{l}\text { molecular } \\
\text { weight }(D a)\end{array}$ & 12143.64 \\
\hline multicomponent & no \\
\hline
\end{tabular}

Lensen, M. C., van Dingenen, S. J. T., Elemans, J. A. A. W., Dijkstra, H. P., van Klink, G. P. M., van Koten, G., Gerritsen, J. W., Speller, S., nolte, R. J. M. \& Rowan, A. E. Chem. Commun. 2004 762-763.

\section{Record 102}

5,5-Dihydroxymethyl-3,3-dipentyl-

2,2':5',2:5,2"':5"',2-quinquethiophene (3b)

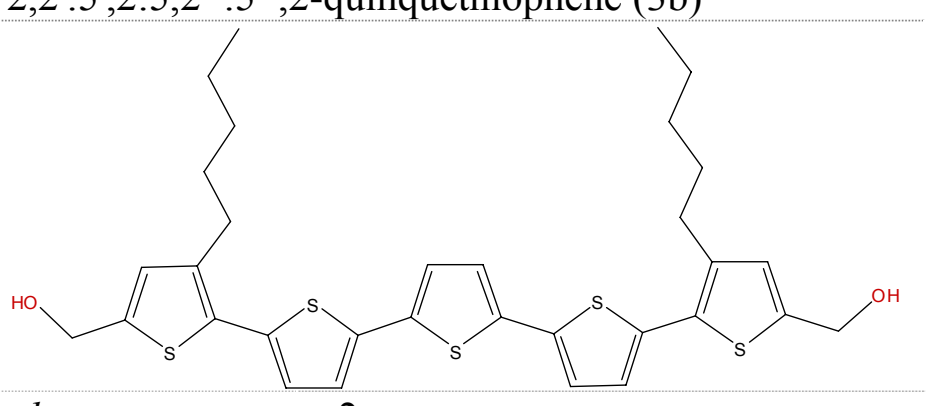

plane group $\quad p 2$

crystal class oblique

$\begin{array}{ll}Z & 2 \\ Z^{\prime} & 1\end{array}$

molecular $\quad \mathrm{C}(2 \mathrm{v})$

symmetry

site symmetry 1

molecular $\quad 612.95$

weight (Da)

multicomponent no

Stecher, R., Gompf, B., Münter, J. S. R. \& Effenberger, F. Adv. Mater. 1999 11, 927-931.

\section{Record 103}

4-(4'-n-propylcyclohexyl)-cyanocyclohexane (CCH3)

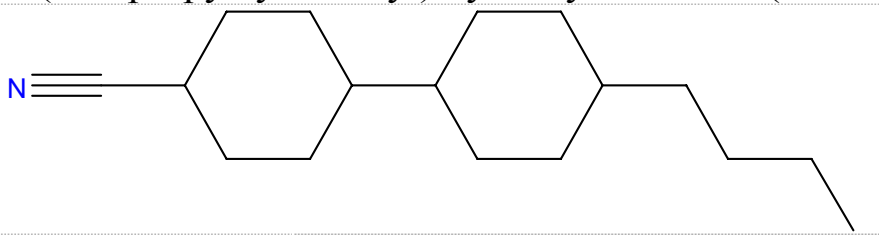

site symmetry 1

molecular 247.42

weight (Da)

multicomponent no

Spong, J. K., Mizes, H. A., Lacomb, L. J., Dovek, M.

M., Frommer, J. E. \& Foster, J. S. Nature 1989 338, 137-139.

\section{Record 104}

hexadecanoic acid (aka palmitic acid)

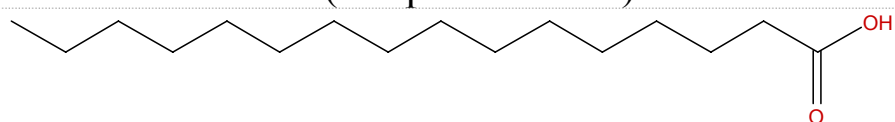

plane group $\quad p 2$

crystal class oblique

$Z \quad 2$

$Z^{\prime} \quad 1$

molecular $\quad \mathrm{C}(\mathrm{s})$

symmetry

site symmetry 1

molecular $\quad 256.42$

weight (Da)

multicomponent no

Yablon, D. G., Giancarlo, L. C. \& Flynn, G. W. J. Phys. Chem. B 2000 104, 7627-7635.

\section{Record 105}

tetracosane

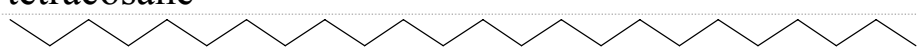

\begin{tabular}{ll} 
plane group & $p 2$ \\
crystal class & oblique \\
\hline$Z$ & 1 \\
\hline$Z^{\prime}$ & 0.5
\end{tabular}

molecular $\quad \mathrm{C}(2 \mathrm{~h})$

symmetry

site symmetry 2

molecular $\quad 338.65$

weight (Da)

multicomponent no

Askadskaya, L. \& Rabe, J. P. Phys. Rev. Lett. 1992 69, 1395-1398.

\section{Record 106}

$3 \mathrm{c}$

\begin{tabular}{ll} 
plane group & $p 2$ \\
\hline crystal class & oblique \\
\hline$Z$ & 2 \\
\hline$Z^{\prime}$ & 1 \\
\hline $\begin{array}{l}\text { molecular } \\
\text { symmetry }\end{array}$ & $\mathrm{C}(\mathrm{s})$ \\
\hline
\end{tabular}




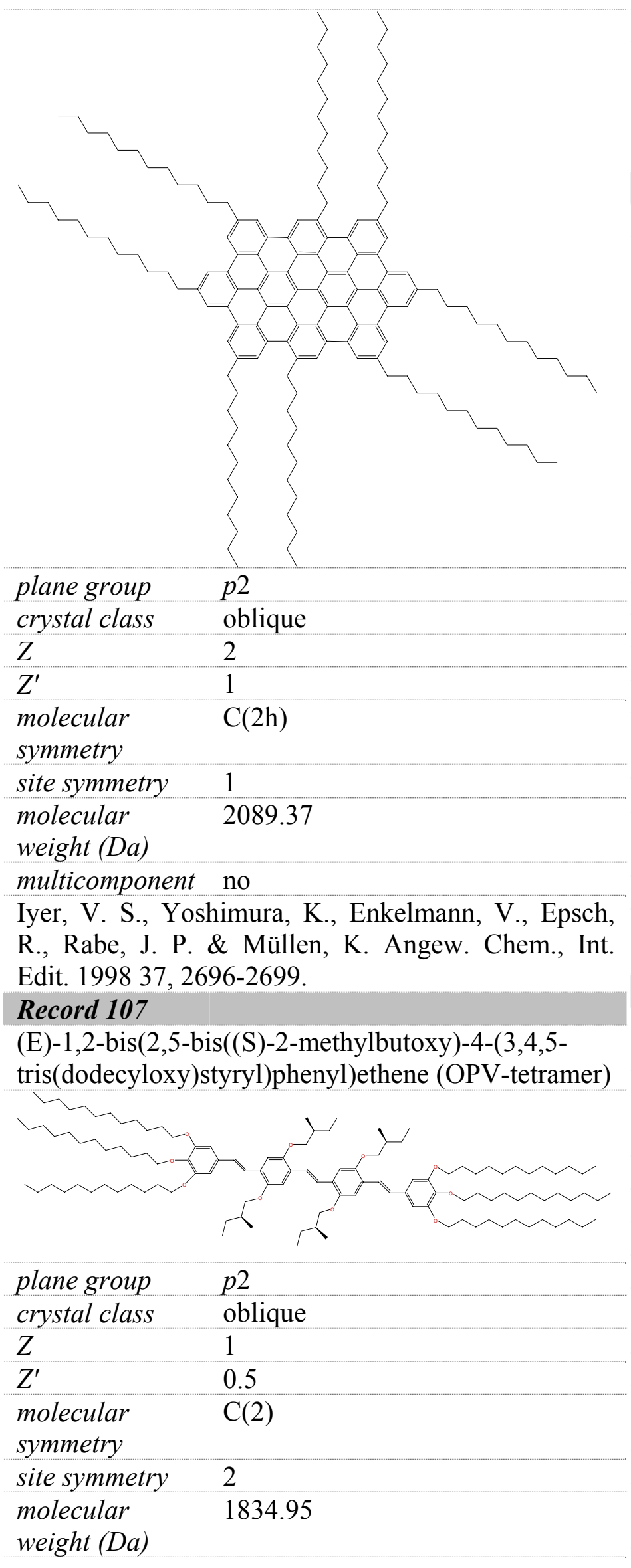

multicomponent no

Gesquière, A., Jonkheijm, P., Schenning, A., MenaOsteritz, E., Bäuerle, P., De Feyter, S., De Schryver, F. C. \& Meijer, E. W. J. Mater. Chem. 2003 13, 2164-2167.

\section{Record 108}

3,3"'-Dihexyl-2,2':5',2":5",2"'-quaterthiophene

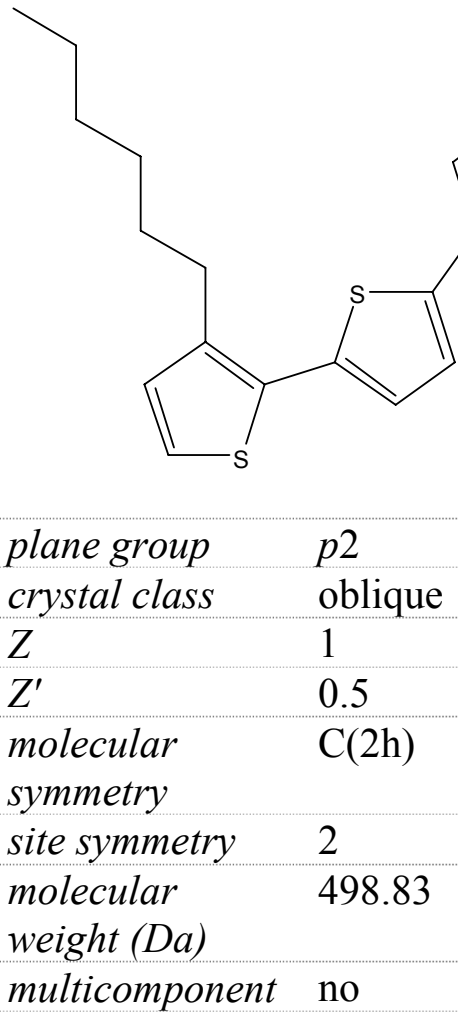

Azumi, R., Götz, G., Debaerdemaeker, T. \& Bäuerle, P. Chem.-Eur. J. 2000 6, 735-744.

\section{Record 109}

steric acid

1-octadecanoic acid

plane group
crystal class




\section{Record 110}

S-2-bromohexadecanoic acid

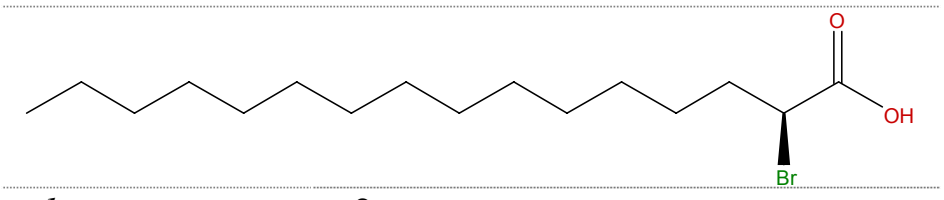

\begin{tabular}{ll} 
plane group & $p 2$ \\
crystal class & oblique \\
\hline$Z$ & 2 \\
\hline$Z^{\prime}$ & 1 \\
$\begin{array}{l}\text { molecular } \\
\text { symmetry }\end{array}$ & $\mathrm{C}(1)$ \\
site symmetry & 1 \\
$\begin{array}{l}\text { molecular } \\
\text { weight }(\mathrm{Da})\end{array}$ & 335.32 \\
\hline
\end{tabular}

multicomponent no

Fang, H. B., Giancarlo, L. C. \& Flynn, G. W. J. Phys. Chem. B 1998 102, 7311-7315.

\section{Record 111}

behenic acid

$\begin{aligned} & \text { plane group } \\ & \text { crystal class }\end{aligned}$
$\begin{aligned} & Z \\ & Z^{\prime}\end{aligned}$
$\begin{aligned} & \text { molecular } \\ & \text { symmetry }\end{aligned}$
$\begin{aligned} & \text { site symmetry } \\ & \text { molecular } \\ & \text { weight (Da) }\end{aligned}$
$\begin{aligned} & \text { multicomponent } \\ & \text { Hibino, M., Sumi, A., Tsuchiya, H. \& Hatta, I. J. }\end{aligned}$
Phys. Chem. B 1998 102, 4544-4547.

\section{Record 112}

cis-1

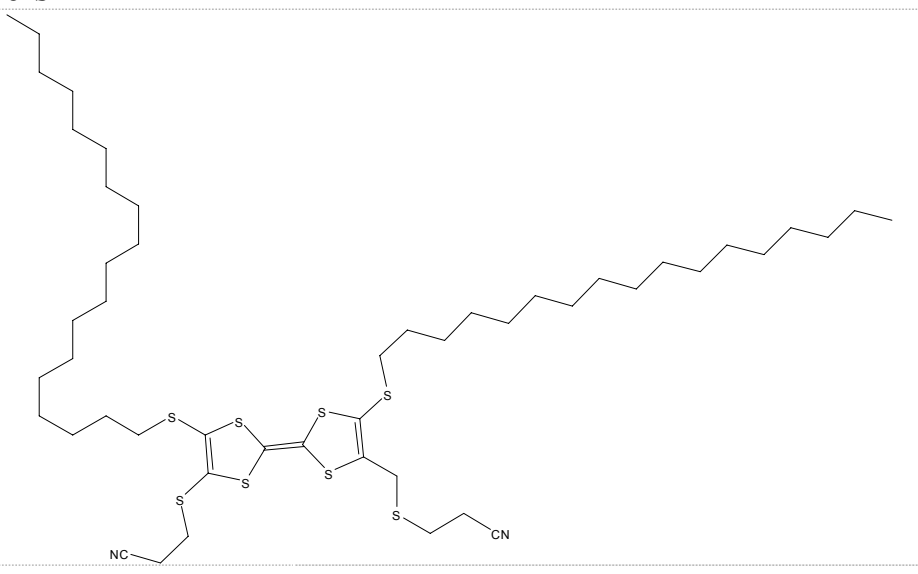

plane group crystal class oblique

$Z \quad 2$

$Z^{\prime} \quad 1$

molecular $\quad \mathrm{C}(2 \mathrm{v})$

symmetry

site symmetry 1

molecular $\quad 943.70$

weight (Da)

multicomponent no

Gomar-Nadal, E., Abdel-Mottaleb, M. M. S., De Feyter, S., Veciana, J., Rovira, C., Amabilino, D. B. \& De Schryver, F. C. Chem. Commun. 2003 906907.

\section{Record 113}

$3 \mathrm{~b}$

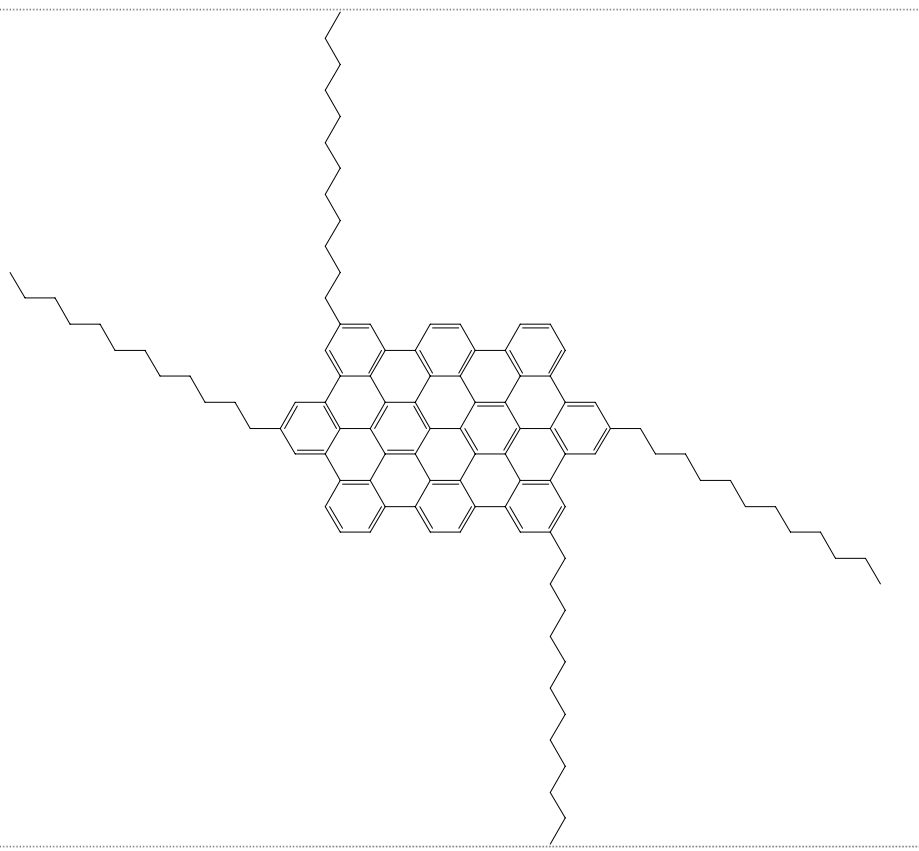

plane group $\quad p 2$

crystal class oblique

$\begin{array}{ll}Z & 2\end{array}$

$Z^{\prime} \quad 1$

molecular $\quad \mathrm{C}(2 \mathrm{~h})$

symmetry

site symmetry 22

molecular $\quad 1416.09$

weight (Da)

multicomponent no

Iyer, V. S., Yoshimura, K., Enkelmann, V., Epsch, R., Rabe, J. P. \& Müllen, K. Angew. Chem., Int. Edit. 1998 37, 2696-2699.

\section{Record 114}


steric acid

1-octadecanoic acid

(1)

plane group $\quad p 2$

crystal class oblique

$Z$ Z

$Z^{\prime} \quad 1$

molecular $\quad \mathrm{C}(\mathrm{s})$

symmetry

site symmetry 1

molecular $\quad 284.48$

weight (Da)

multicomponent no

Olson, J. A. \& Bühlmann, P. Anal. Chem. 2003 75, 1089-1093.

\section{Record 115}

trans - alpha

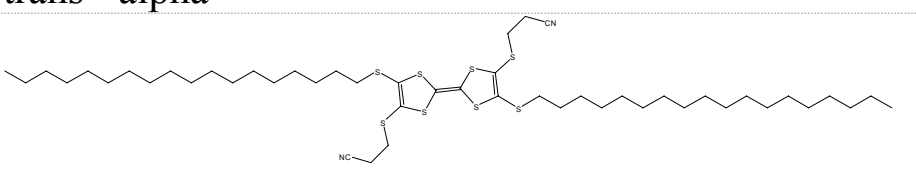

plane group $\quad p 2$

crystal class oblique

Z 1

$Z^{\prime} \quad 0.5$

molecular $\quad \mathrm{C}(2 \mathrm{~h})$

symmetry

site symmetry 1

molecular $\quad 943.70$

weight (Da)

multicomponent no

Gomar-Nadal, E., Abdel-Mottaleb, M. M. S., De

Feyter, S., Veciana, J., Rovira, C., Amabilino, D. B.

\& De Schryver, F. C. Chem. Commun. 2003 906907.

\section{Record 116}

bis(4-dibutylamino-2-phenyl)squaraine

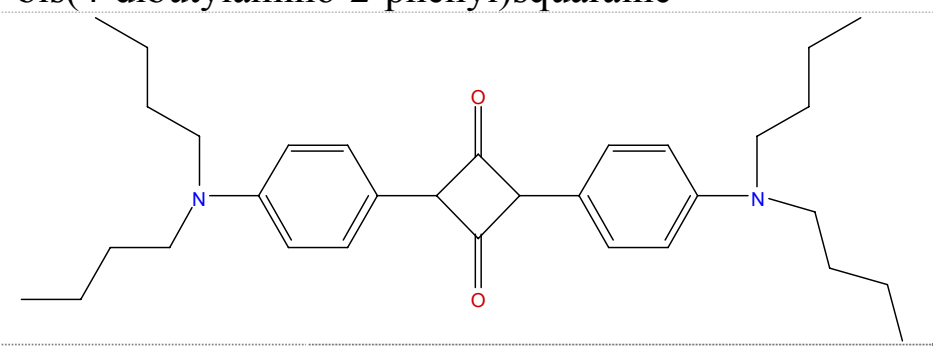

plane group $\quad p 2$

crystal class

oblique

Z

$\begin{array}{ll}Z^{\prime} & 0.5 \\ \text { molecular } & \mathrm{C}(2 \mathrm{v}) \\ \text { symmetry } & \\ \text { site symmetry } & 2 \\ \begin{array}{l}\text { molecular } \\ \text { weight }(\mathrm{Da})\end{array} & 490.72 \\ \text { multicomponent } & \text { no }\end{array}$

Yasuda, S. \& Shigekawa, H. Jpn. J. Appl. Phys., Part

12003 42, 4901-4904.

\section{Record 117}

2,5,8,11,14,17-hexadodecylhexa-peri-hexa-

benzocoronene2a (1b)

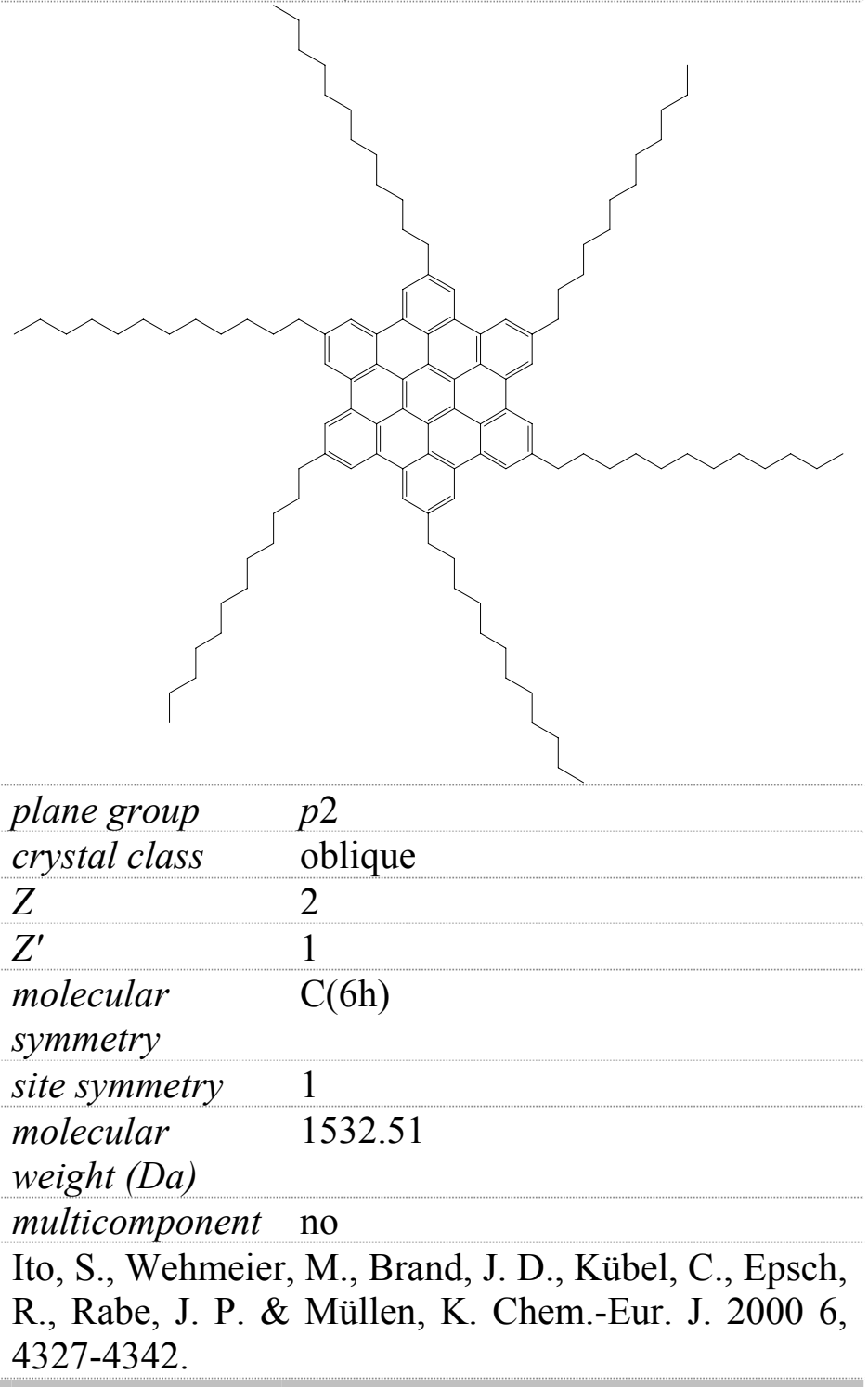

\section{Record 118}

racemic - 12-hydroxy-octadecanoic acid 


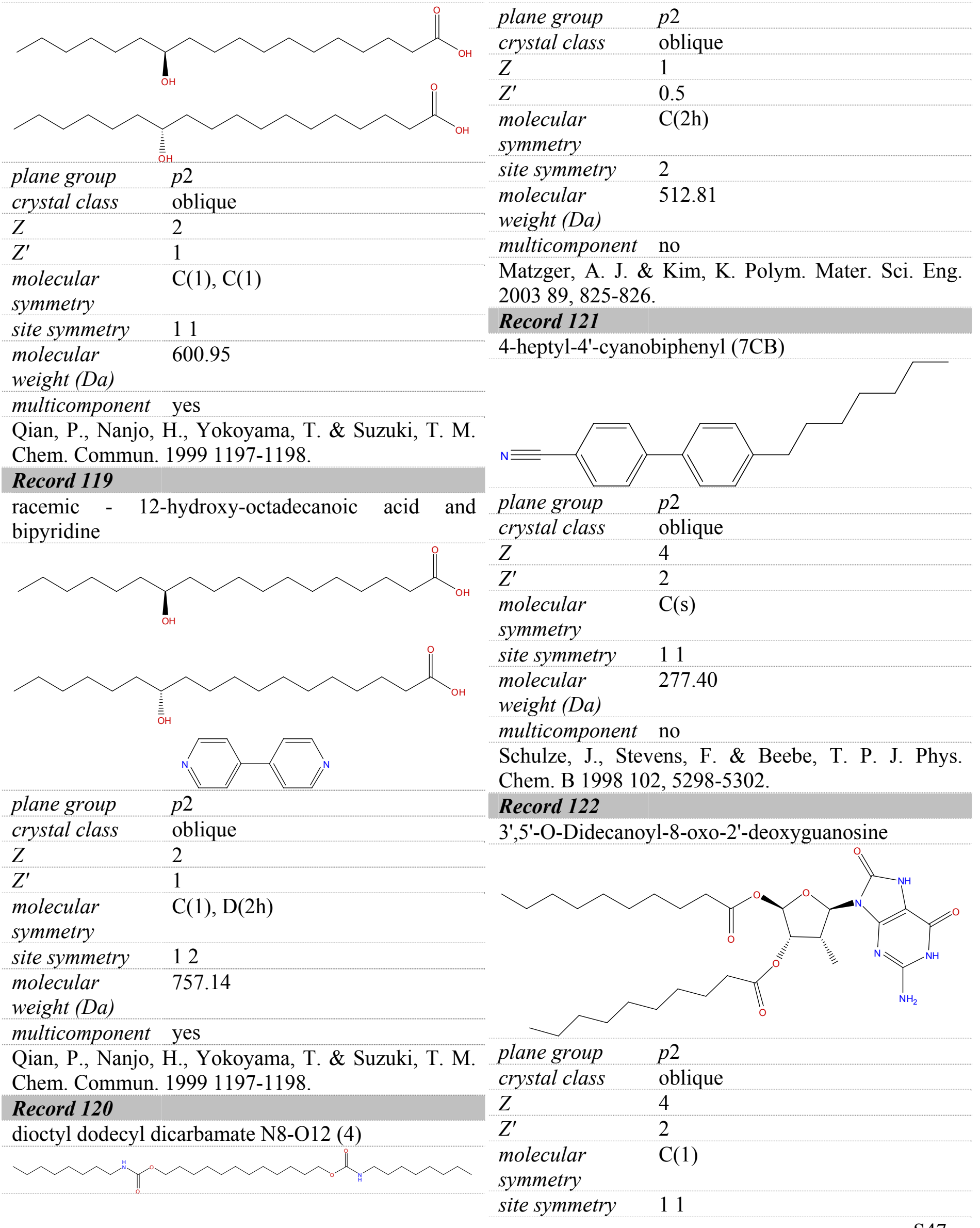


molecular

591.74

weight (Da)

multicomponent no

Giorgi, T., Lena, S., Mariani, P., Cremonini, M. A.,

Masiero, S., Pieraccini, S., Rabe, J. P., Samorí, P.,

Spada, G. P. \& Gottarelli, G. J. Am. Chem. Soc. 2003

125, 14741-14749.

Record 123

Ester-23; 1,3-di-(1-heneicosyl) isophthalate

plane group $\quad p 2$

crystal class oblique

Z 2

$Z^{\prime}-1$

molecular $\mathrm{C}(2 \mathrm{v})$

symmetry

site symmetry 1

molecular 755.25

weight (Da)

multicomponent no

Plass, K. E., Kim, K. \& Matzger, A. J. "Twodimensional crystallization: self-assembly, pseudopolymorphism, and symmetry-independent molecules." J. Am. Chem. Soc. 2004 126, 9042-9053.

\section{Record 124}

Thioester-20

1,3-Benzenedicarbothioic acid, S,S-dioctadecyl ester

\begin{tabular}{ll} 
plane group & $p 2$ \\
crystal class & oblique \\
\hline$Z$ & 2 \\
\hline$Z^{\prime}$ & 1 \\
$\begin{array}{l}\text { molecular } \\
\text { symmetry }\end{array}$ & $\mathrm{C}(2 \mathrm{v})$ \\
site symmetry & 1 \\
$\begin{array}{l}\text { molecular } \\
\text { weight }(\text { Da })\end{array}$ & 703.22 \\
multicomponent & no
\end{tabular}

Plass, K. E., Kim, K. \& Matzger, A. J. J. Am. Chem.

Soc. 2004 126, 9042-9053.

\section{Record 125}

Ester-19; 1,3-di-(1-heptadecyl) isophthalate

$\begin{array}{ll}\text { plane group } & p 2 \\ \text { crystal class } & \text { oblique } \\ Z & 2 \\ Z & 1 \\ Z^{\prime} & \mathrm{C}(2 \mathrm{v}) \\ \text { molecular } & \\ \text { symmetry } & 1 \\ \text { site symmetry } & 643.03 \\ \begin{array}{l}\text { molecular } \\ \text { weight }(\text { Da })\end{array} & \text { no } \\ \text { multicomponent } & \end{array}$

Plass, K. E., Kim, K. \& Matzger, A. J. J. Am. Chem. Soc. 2004 126, 9042-9053.

\section{Record 126}

tpy-O-C12

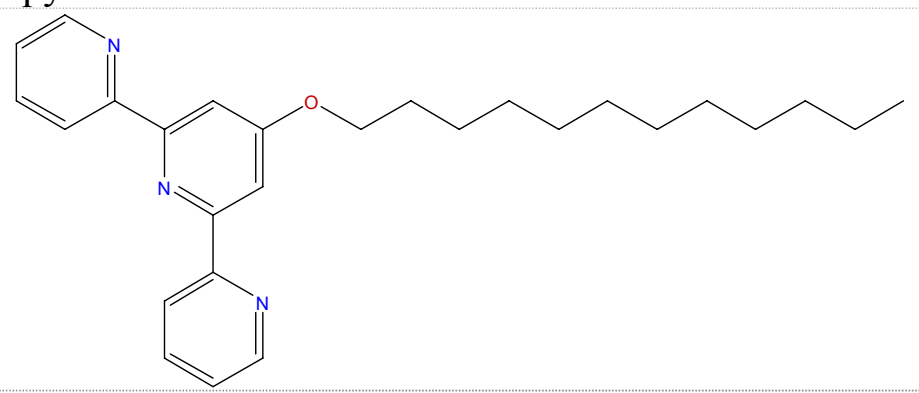

plane group $\quad p 2$

crystal class oblique

Z 2

$Z^{\prime} \quad 1$

molecular $\mathrm{C}(\mathrm{s})$

symmetry

site symmetry 1

molecular $\quad 417.59$

weight (Da)

multicomponent no

Wouters, D., Höppener, S., Lunkwitz, R., Chi, L. F., Fuchs, H. \& Schubert, U. S. Adv. Funct. Mater. 2003 13, 277-280.

\section{Record 127}

alpha-[[4-[(N,N-

Dimethylcarbamoyl)thio]phenyl]ethynyl]--[4-[(N,N-

dimethylcarbamoyl)thio]phenyl]-ter[(2,5-

dihexylphenylene-1,4)ethynylene)] 

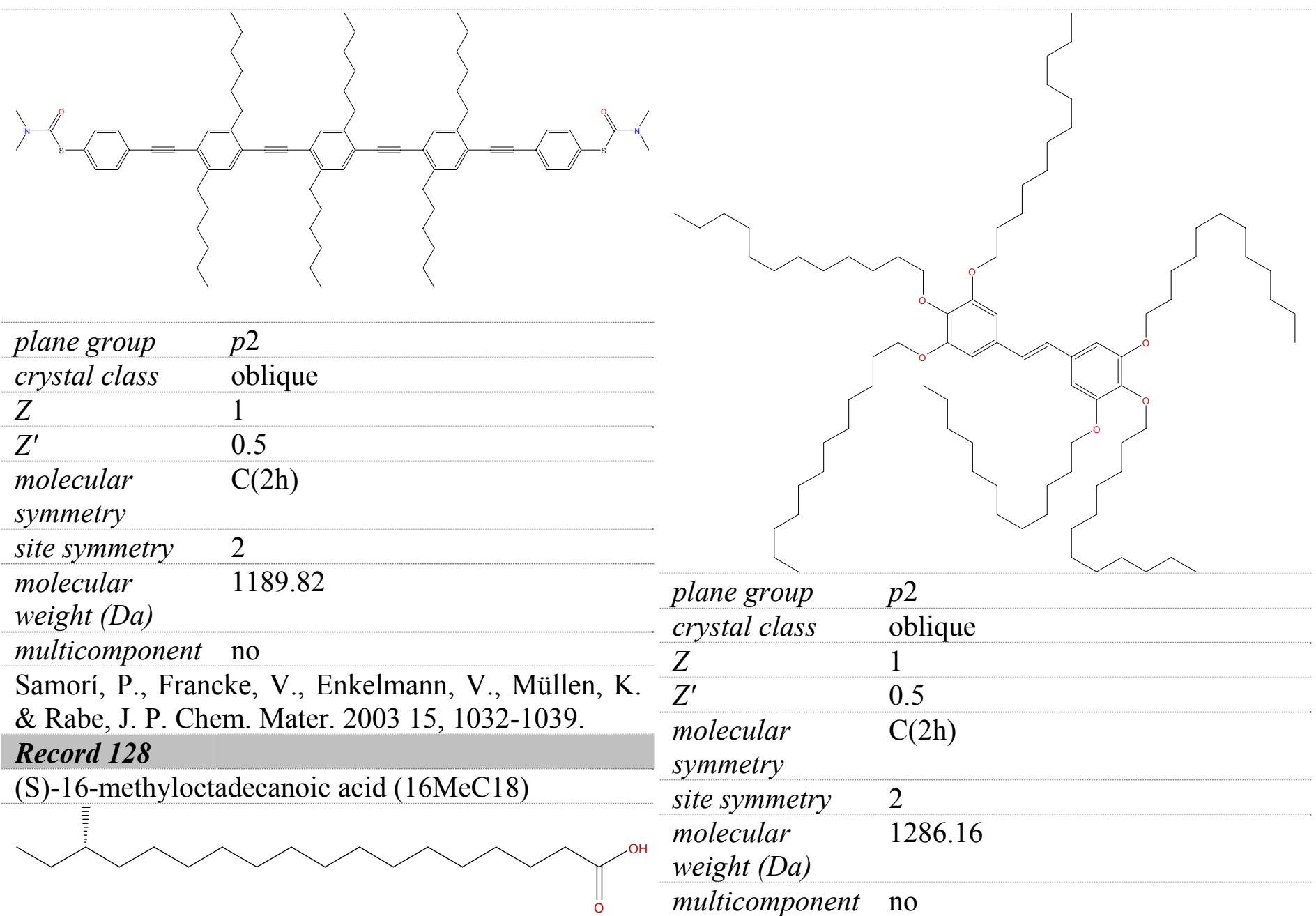

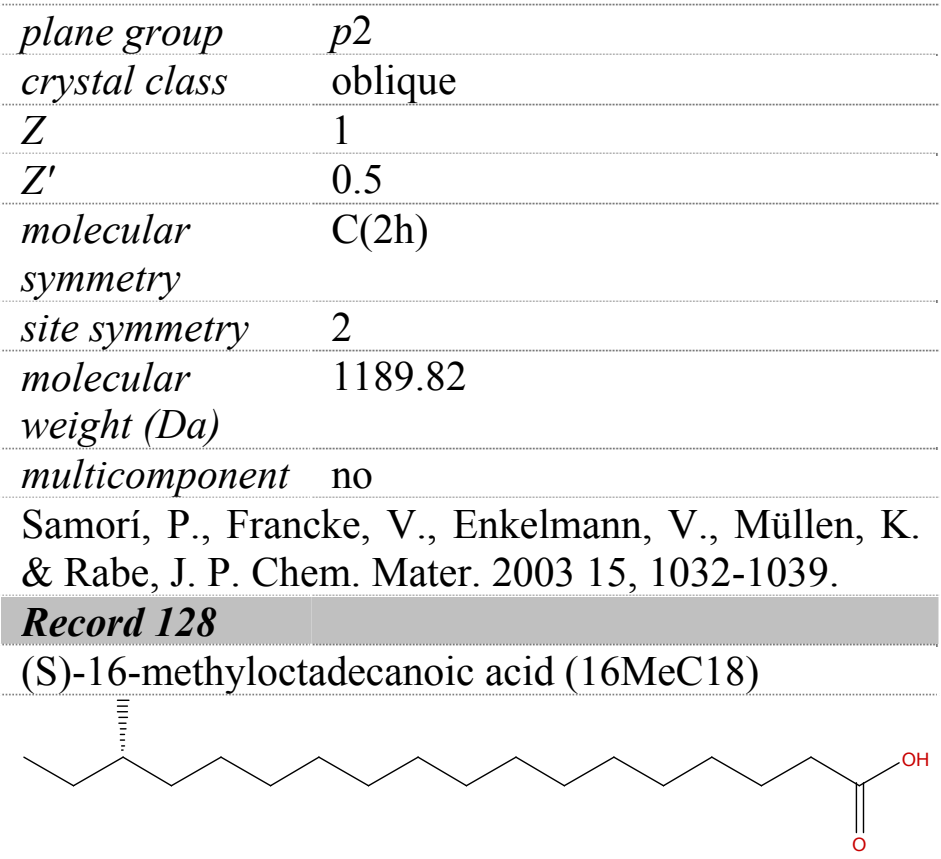

$\begin{array}{ll}\text { plane group } & p 2 \\ \text { crystal class } & \text { oblique } \\ Z & 2 \\ Z \begin{array}{l}Z^{\prime} \\ \text { molecular }\end{array} & 1 \\ \text { symmetry } & \mathrm{C}(1) \\ \text { site symmetry } & 1 \\ \begin{array}{l}\text { molecular } \\ \text { weight (Da) }\end{array} & 298.50 \\ \text { multicomponent } & \text { no } \\ \text { Qian, P., Nanjo, H., Sanada, N., Yokoyama, T. \& } \\ \text { Suzuki, T. M. Chem. Lett. 2000 1118-1119. }\end{array}$

\section{Record 129}

(E)-1,2-bis(3,4,5-tris(dodecyloxy)phenyl)ethene (OPV-dimer)

Gesquière, A., Jonkheijm, P., Schenning, A., MenaOsteritz, E., Bäuerle, P., De Feyter, S., De Schryver, F. C. \& Meijer, E. W. J. Mater. Chem. 2003 13, 2164-2167.

\section{Record 130}

(E)-1,2-bis(4-(2,5-bis((S)-2-methylbutoxy)-4-(3,4,5tris(dodecyloxy)styryl)styryl)-2,5-bis((S)-2methylbutoxy)phenyl)ethene (OPV6)

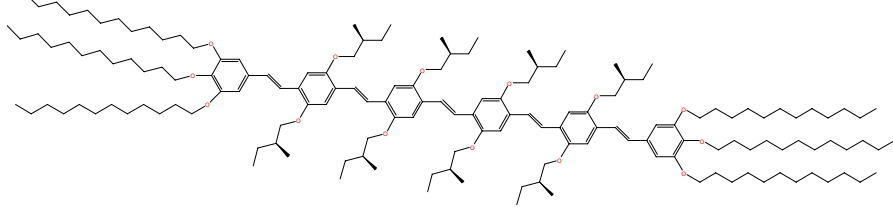

\begin{tabular}{ll} 
plane group & $p 2$ \\
crystal class & oblique \\
$Z$ & 1 \\
$Z^{\prime}$ & 0.5 \\
$\begin{array}{l}\text { molecular } \\
\text { symmetry }\end{array}$ & $\mathrm{C}(2)$ \\
site symmetry & 2 \\
molecular & 2383.75 \\
\hline
\end{tabular}


weight (Da)

multicomponent no

Gesquière, A., Jonkheijm, P., Schenning, A., MenaOsteritz, E., Bäuerle, P., De Feyter, S., De Schryver, F. C. \& Meijer, E. W. J. Mater. Chem. 2003 13, 2164-2167.

\section{Record 131}

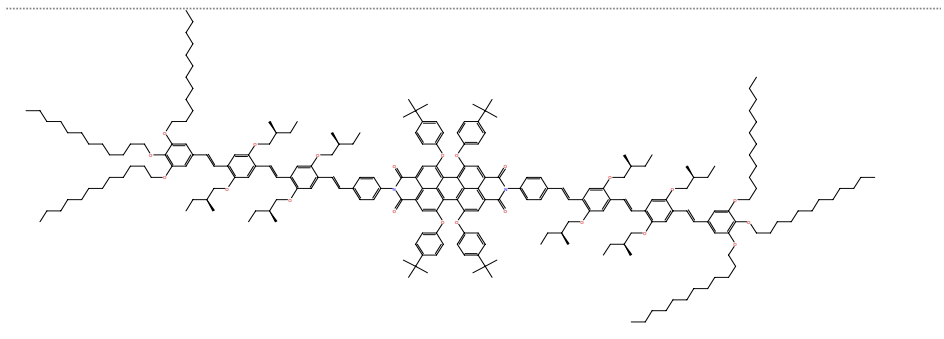

plane group p2

$\begin{array}{ll}\text { crystal class } & \text { oblique } \\ Z & 1 \\ Z^{\prime} & 0.5\end{array}$

molecular

symmetry

$\mathrm{C}(2)$

site symmetry 2

molecular

3543.11

weight (Da)

multicomponent no

Miura, A., Chen, Z., Uji-i, H., De Feyter, S., Zdanowska, M., Jonkheijm, P., Schenning, A. P. H. J., Meijer, E. W., Würthner, F. \& De Schryver, F. C. J. Am. Chem. Soc. 2003 125, 14968-14969.

\section{Record 132}

4-(5,8,11,14,17-pentakis(3,7-dimethyloctyl)$3 \mathrm{a} 1,4,7,9 \mathrm{~b} 1,13,-$

hexahydrohexabenzo[bc,ef,hi,kl,no,qr]coronen-2yl)butyl pyrene-1-carboxylate

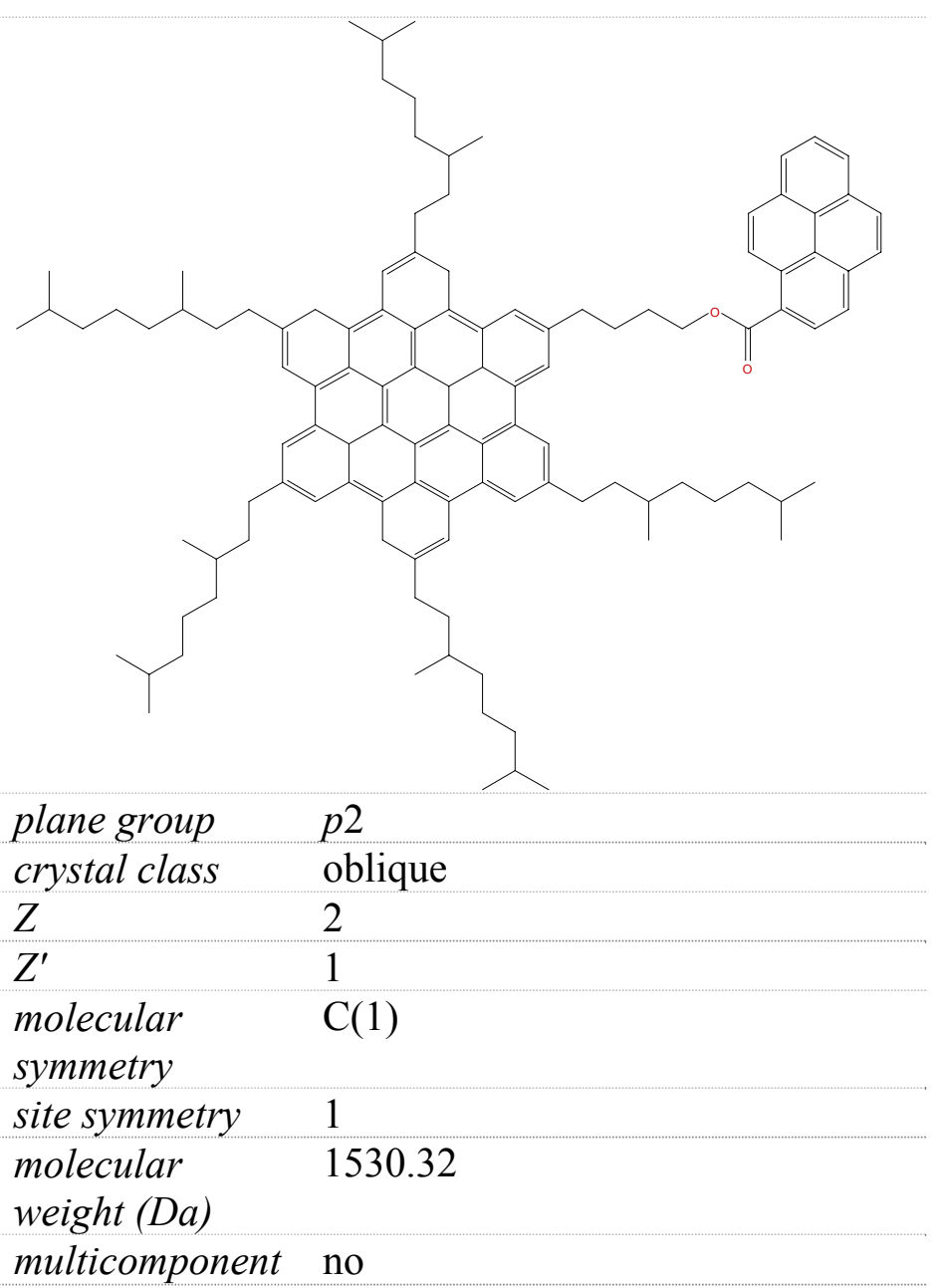

Tchebotareva, N., Yin, X., Watson, M. D., Samorí, P., Rabe, J. P. \& Müllen, K. J. Am. Chem. Soc. 2003 125, 9734-9739.

\section{Record 133}

tridodecylamine (TDA)

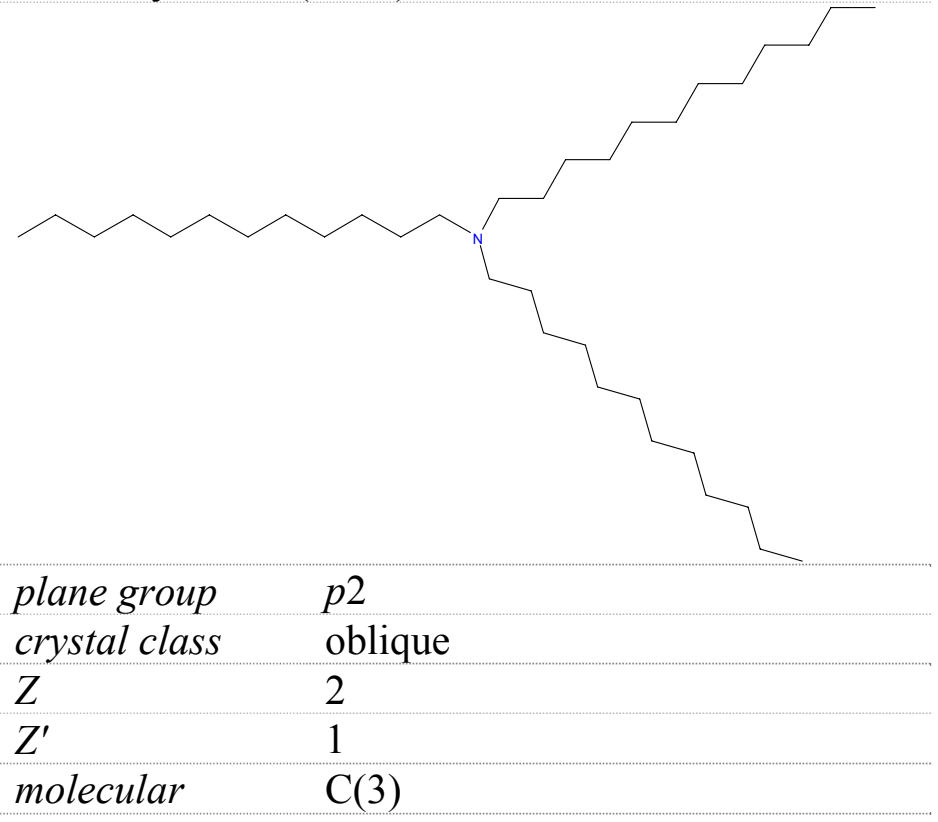


symmetry

site symmetry 1

molecular

521.99

weight (Da)

multicomponent no

Lei, S.-B., Wang, C., Fan, X.-L., Wan, L.-J. \& Bai, C.-L. Langmuir 2003 19, 9759-9763.

\section{Record 134}

tetrakis-(octadecylthio)-tetrathiafulvalene

plane group
crystal class

Uji-i, H., Yoshidome, M., Hobley, J., Hatanaka, K. \& Fukumura, H. Phys. Chem. Chem. Phys. 2003 5, 4231-4235.

\section{Record 136} tpy-O-C18

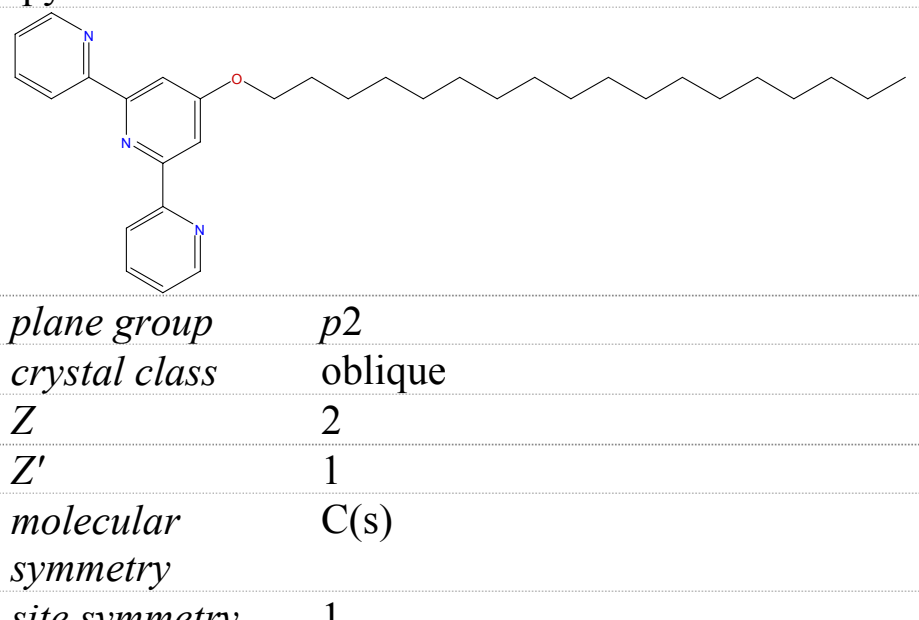

site symmetry 1

molecular $\quad 501.75$

weight (Da)

multicomponent no

Wouters, D., Höppener, S., Lunkwitz, R., Chi, L. F., Fuchs, H. \& Schubert, U. S. Adv. Funct. Mater. 2003 13, 277-280.

\section{Record 137}

16-hydroxyhexadecyl docosanoate

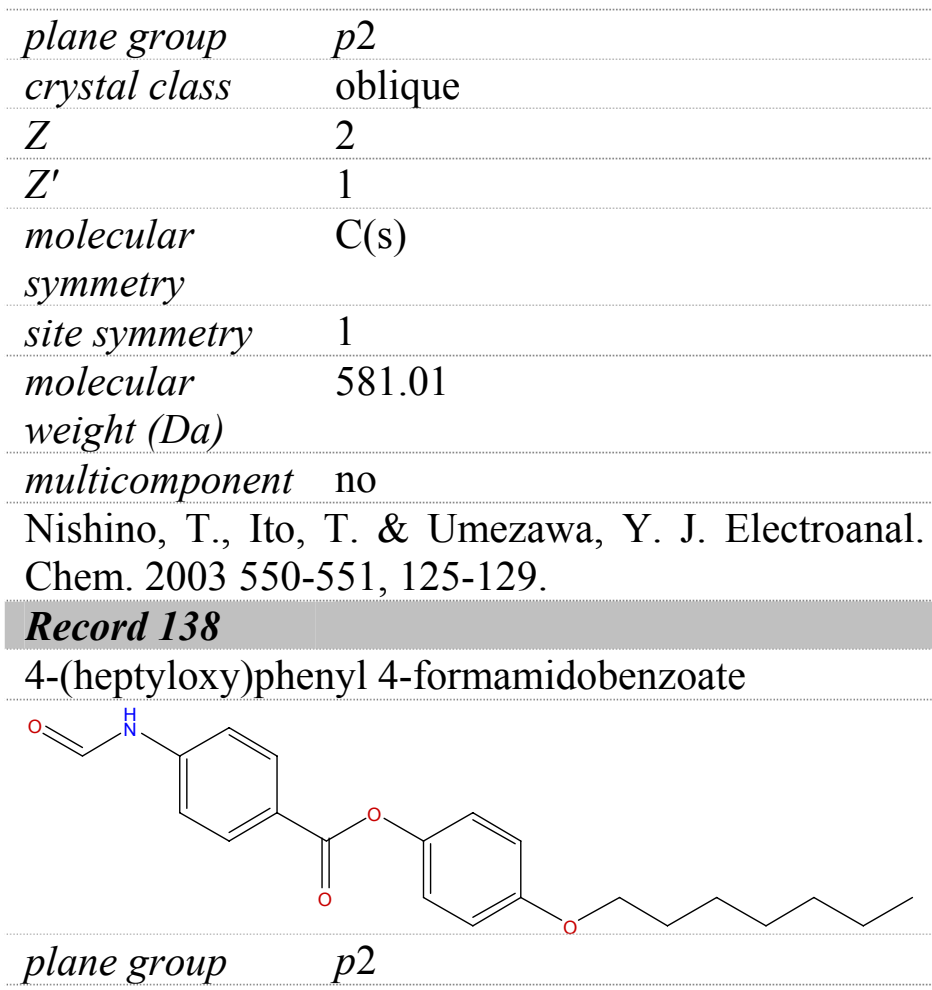




\begin{tabular}{|c|c|c|}
\hline crystal class & oblique & \\
\hline$Z$ & 2 & \\
\hline$Z^{\prime}$ & 1 & \\
\hline $\begin{array}{l}\text { molecular } \\
\text { symmetry }\end{array}$ & $C(1)$ & \\
\hline site symmetry & 1 & \\
\hline $\begin{array}{l}\text { molecular } \\
\text { weight }(D a)\end{array}$ & 355.43 & \\
\hline $\begin{array}{l}\text { multicomponen } \\
\text { De Feyter, S., } \\
\text { D. B., Vecian } \\
\text { Chem., Int. Edi }\end{array}$ & $\begin{array}{l}\text { no } \\
\text { squière, A., Wurst, K., Amabilino, } \\
\text { J. \& De Schryver, F. C. Angew. } \\
001 \text { 40, 3217-3220. }\end{array}$ & \\
\hline $\begin{array}{l}\text { Record } 139 \\
\text { 1,1'-(dodecane- } \\
12 \text { ) }\end{array}$ & 2-diyl)bis(3-dodecylurea) (C12- & \\
\hline 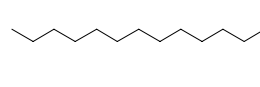 & " & \\
\hline plane group & $p 2$ & \\
\hline crystal class & oblique & \\
\hline$Z$ & 1 & \\
\hline$Z^{\prime}$ & 0.5 & \\
\hline molecular & $C(2 h)$ & \\
\hline $\begin{array}{l}\text { symmetry } \\
\text { site symmetry }\end{array}$ & 2 & plane group $\quad p 2$ \\
\hline molecular & 623.05 & $\begin{array}{ll}\text { crystal class } & \text { oblique } \\
Z & 1\end{array}$ \\
\hline $\begin{array}{l}\text { weight }(\mathrm{Da}) \\
\text { multicomponen }\end{array}$ & no & $Z^{\prime} \quad 0.5$ \\
\hline $\begin{array}{l}\text { De Feyter, S., I } \\
\text { B. Zoriniants. }\end{array}$ & sson, M., Schuurmans, N., Verkuijl, & $\begin{array}{l}\text { molecular } \quad \mathrm{C}(2) \\
\text { symmetry }\end{array}$ \\
\hline $\begin{array}{l}\text { B., Zoriniants, } \\
\text { M., van Esch, }\end{array}$ & $\begin{array}{l}\text { Gesquiere, A., Abdel-Mottaleb, M. } \\
\text { Feringa, B. L., van Stam, J. \& De }\end{array}$ & site symmetry 2 \\
\hline Schryver, F. Cl & n.-Eur. J. 2003 9, 1198-1206. & $\begin{array}{ll}\text { molecular } & 1806.69 \\
\text { weight }(D a) & \end{array}$ \\
\hline Record 140 & $5 \operatorname{lin}(25.50)$ & multicomponent no \\
\hline $\begin{array}{l}(2 \mathrm{~S}, 3 \mathrm{~S})-2,3-\mathrm{bis} \\
\text { bis(octyloxy)b } \\
\text { tetramethylbut: }\end{array}$ & $\begin{array}{l}\text { 5-bis(3,5- } \\
\text { yloxy)benzyloxy)-N1,N1,N4,N4- } \\
\text {-1,4-diamine }\end{array}$ & $\begin{array}{l}\text { Widmer, I., Hubler, U., Stöhr, M., Merz, L., } \\
\text { Güntherodt, H. J., Hermann, B. A., Samorí, P., Rabe, } \\
\text { J. P., Rheiner, P. B., Greiveldinger, G. \& Murer, P. } \\
\text { Helv. Chim. Acta } 2002 \text { 85, 4255-4263. }\end{array}$ \\
\hline & & Record 141 \\
\hline & & $\begin{array}{l}\text { bis[1-(3',4',5'-trioctyl-oxylphenyl)-3-(4'- } \\
\text { octyloxphenyl)propane-1,3-ionate]-palladium(II) }\end{array}$ \\
\hline
\end{tabular}




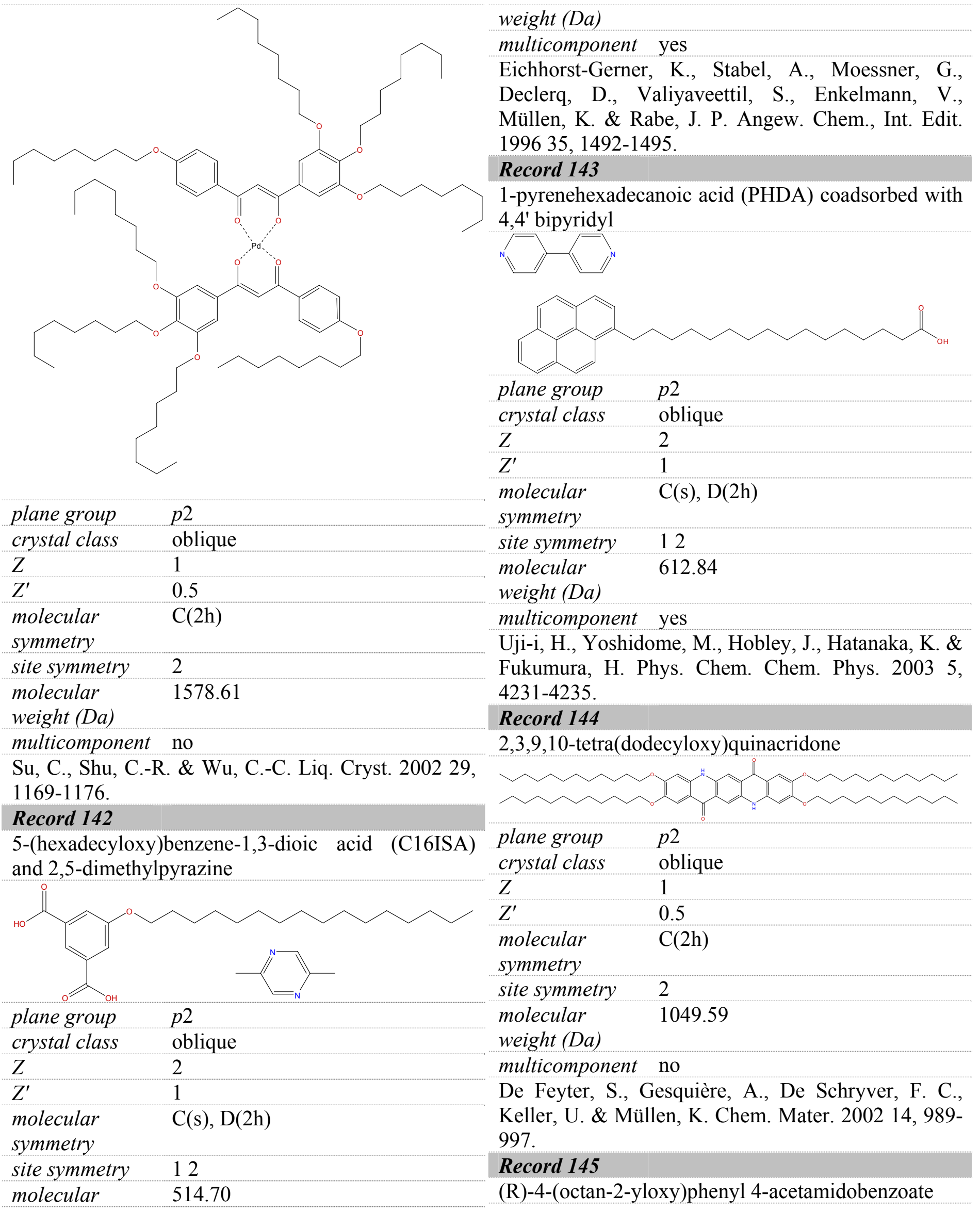




$\begin{array}{ll}\text { plane group } & \text { oblique } \\ \text { crystal class } & 2 \\ Z & 1 \\ Z^{\prime} & \mathrm{C}(1) \\ \text { molecular } & 1 \\ \text { symmetry } & 383.48 \\ \text { site symmetry } & \text { no } \\ \text { molecular } & \text { weight (Da) } \\ \text { multicomponent } & \text { no } \\ \text { De Feyter, S., Gesquìre, A., Wurst, K. Amabilino, }\end{array}$

De Feyter, S., Gesquière, A., Wurst, K., Amabilino, D. B., Veciana, J. \& De Schryver, F. C. Angew. Chem., Int. Edit. 2001 40, 3217-3220.

\section{Record 146}

Por-Ni Ni(II)

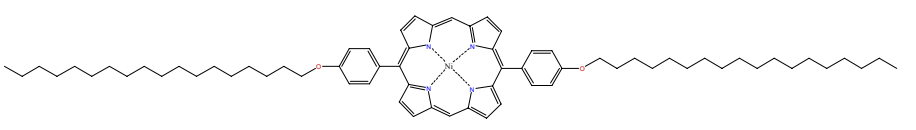

\begin{tabular}{ll} 
plane group & $p 2$ \\
crystal class & oblique \\
$Z Z$ & 1 \\
\hline$Z^{\prime}$ & 0.5 \\
\hline $\begin{array}{l}\text { molecular } \\
\text { symmetry }\end{array}$ & $\mathrm{C}(2 \mathrm{~h})$ \\
site symmetry & 2 \\
$\begin{array}{l}\text { molecular } \\
\text { weight }(\mathrm{Da})\end{array}$ & 1056.18 \\
multicomponent & no \\
\hline
\end{tabular}

Ohshiro, T., Ito, T., Bühlmann, P. \& Umezawa, Y. Anal. Chem. 2001 73, 878-883.

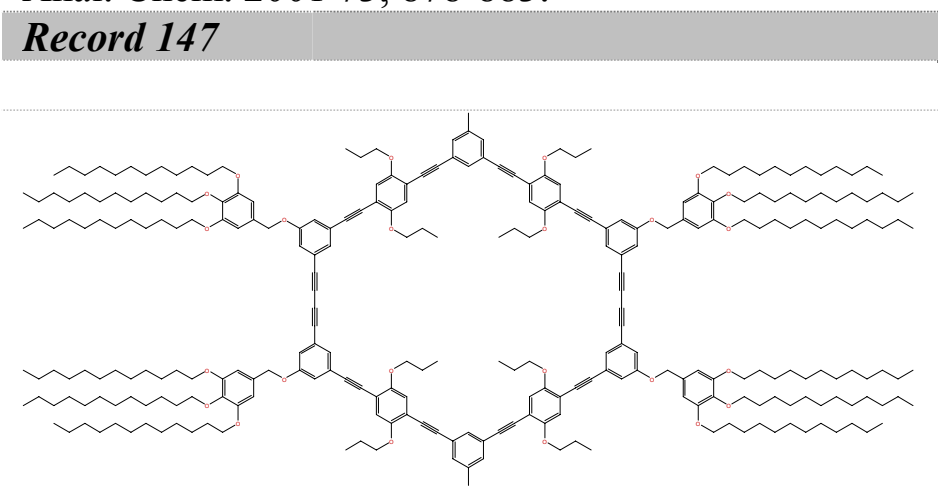

\begin{tabular}{|c|c|}
\hline plane group & $p 2$ \\
\hline crystal class & oblique \\
\hline$Z$ & 1 \\
\hline$Z^{\prime}$ & 0.5 \\
\hline $\begin{array}{l}\text { molecular } \\
\text { symmetry }\end{array}$ & $\mathrm{D}(2 \mathrm{~h})$ \\
\hline site symmetry & 2 \\
\hline $\begin{array}{l}\text { molecular } \\
\text { weight }(\mathrm{Da})\end{array}$ & 4178.21 \\
\hline \multicolumn{2}{|c|}{ multicomponent no } \\
\hline \multicolumn{2}{|c|}{$\begin{array}{l}\text { Höger, S., Bonrad, K., Mourran, A., Beginn, U. \& } \\
\text { Möller, M. J. Am. Chem. Soc. } 2001 \text { 123, 5651-5659. }\end{array}$} \\
\hline \multicolumn{2}{|l|}{ Record 148} \\
\hline \multicolumn{2}{|c|}{$\begin{array}{l}\text { 2,5,8,11,14,17-hexakis(4- } \\
\text { dodecylphenyl)hexabenzo[bc,ef,hi,kl,no,qr]coronene }\end{array}$} \\
\hline
\end{tabular}

$\begin{array}{ll}\text { plane group } & p 2 \\ \text { crystal class } & \text { oblique } \\ Z & 1 \\ Z^{\prime} & 0.5\end{array}$

molecular $\mathrm{C}(6 \mathrm{~h})$

symmetry

site symmetry 2

molecular $\quad 1989.08$

weight (Da)

multicomponent no

Samorí, P., Fechtenkötter, A., Jäckel, F., Böhme, T., Müllen, K. \& Rabe, J. P. J. Am. Chem. Soc. 2001 123, 11462-11467.

\section{Record 149}

PTCDI-C18 


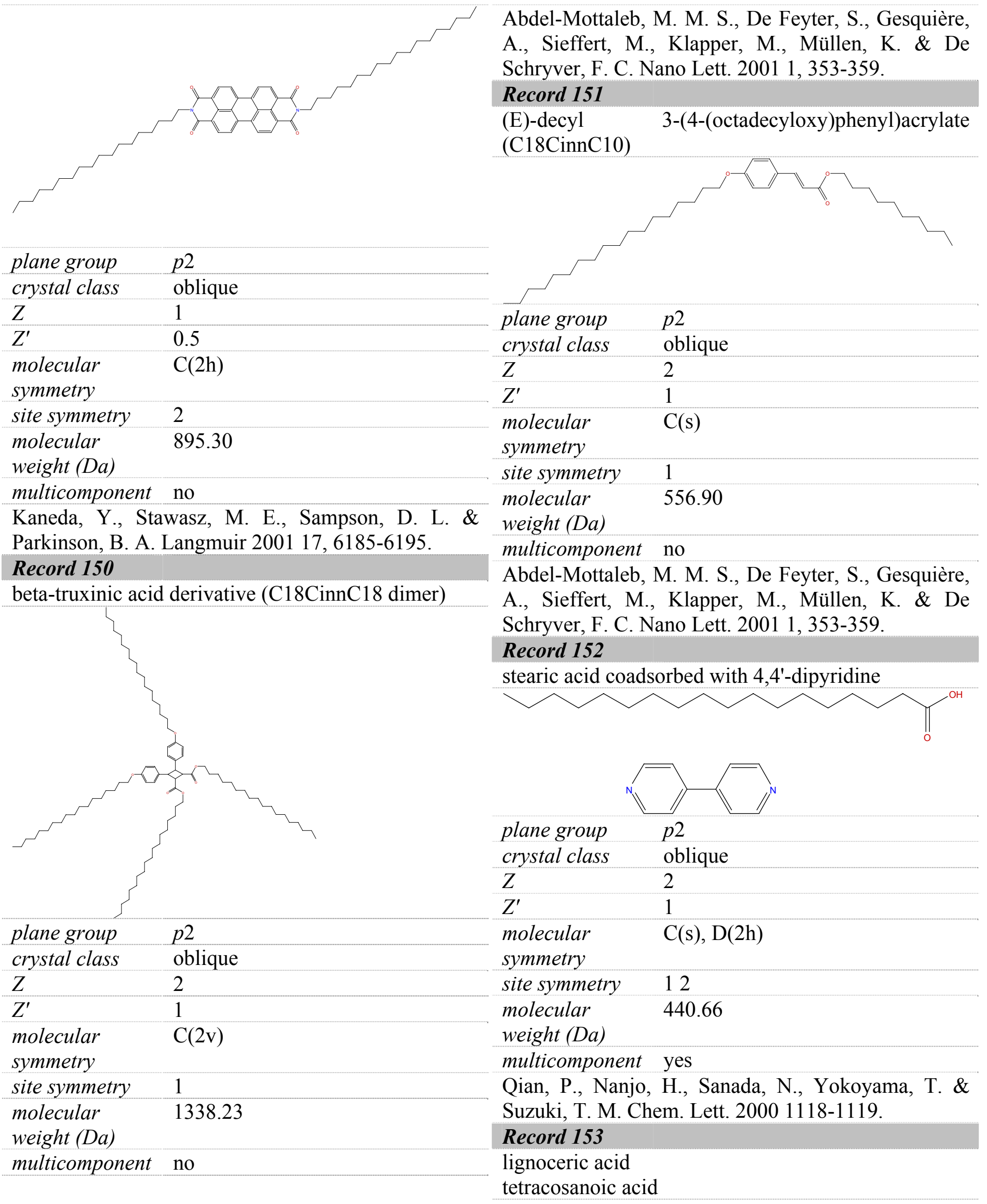




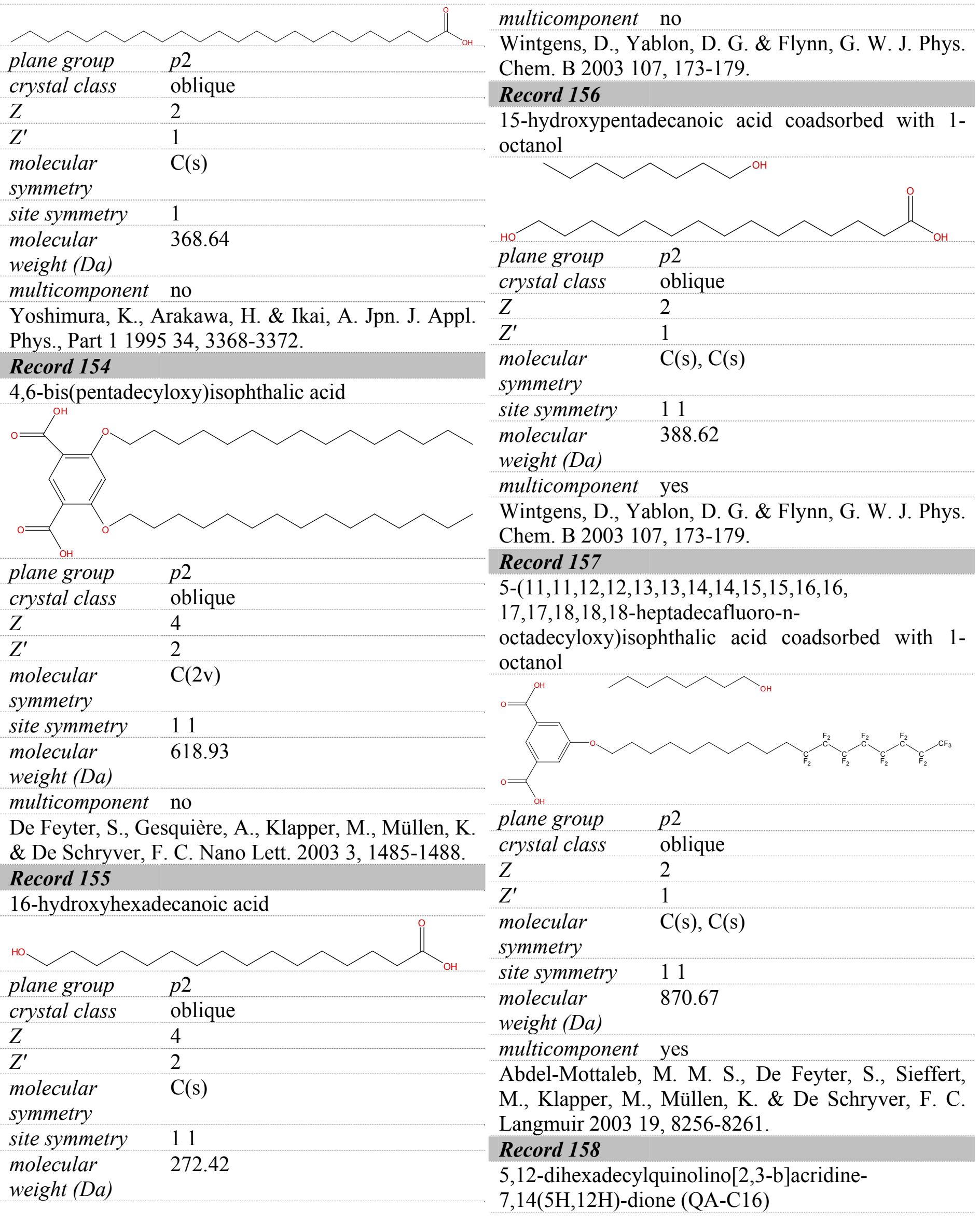




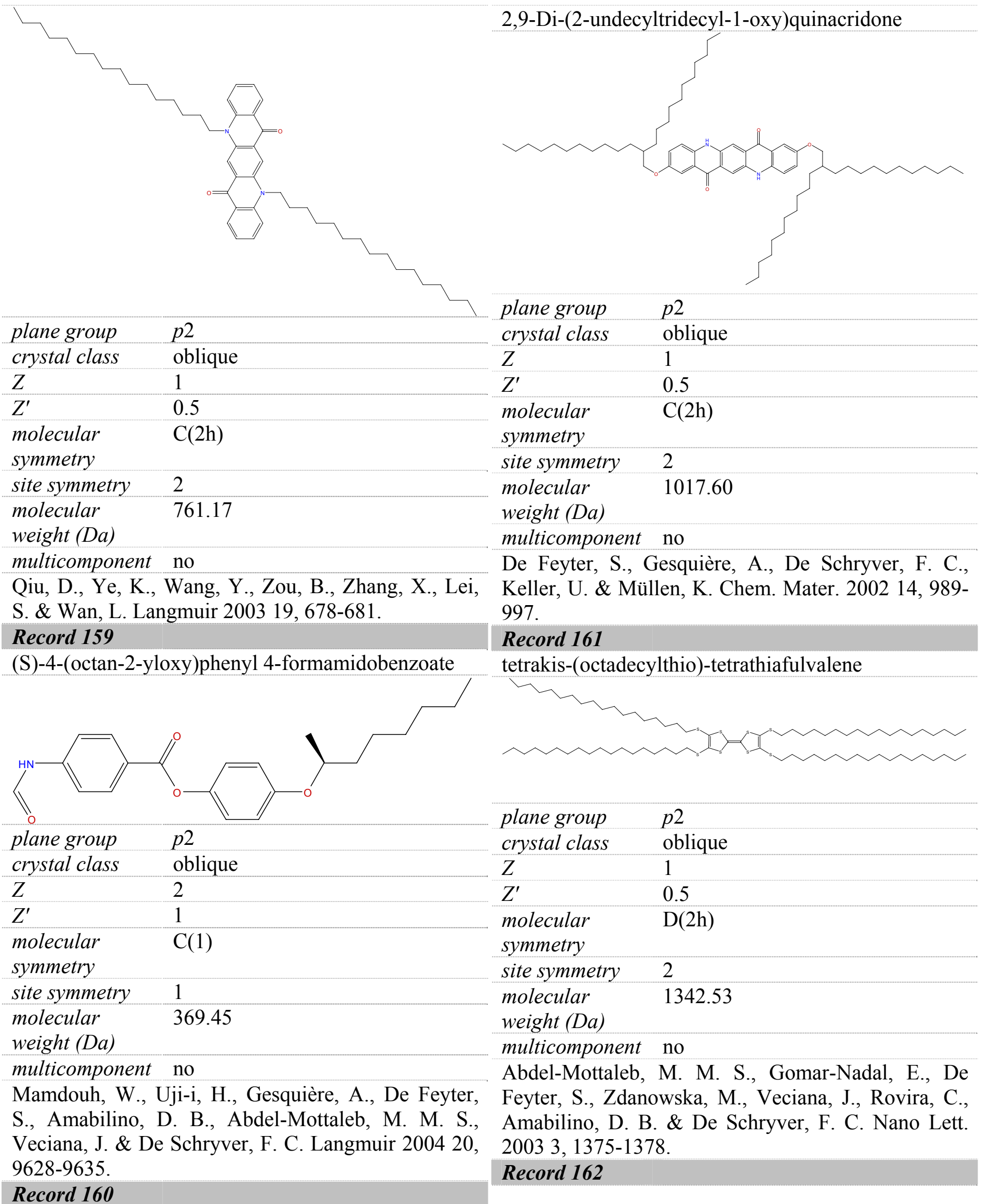


(S)-16-methyloctadecanoic acid (16MeC18) coadsorbed with 4,4'-dipyridine

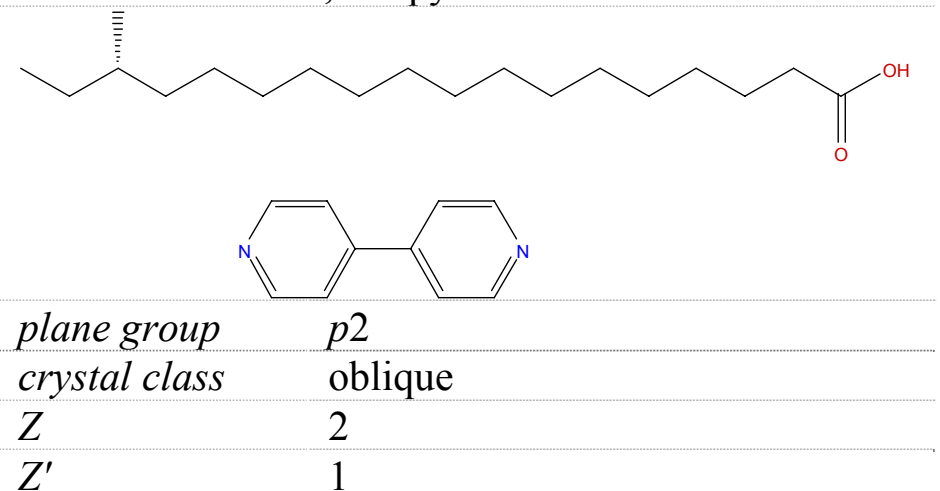

molecular $\quad \mathrm{C}(1), \mathrm{D}(2 \mathrm{~h})$

symmetry

site symmetry 12

molecular $\quad 454.69$

weight (Da)

multicomponent yes

Qian, P., Nanjo, H., Sanada, N., Yokoyama, T. \&

Suzuki, T. M. Chem. Lett. 2000 1118-1119.

\section{Record 163}

4,6-bis(pentadecyloxy)isophthalic acid

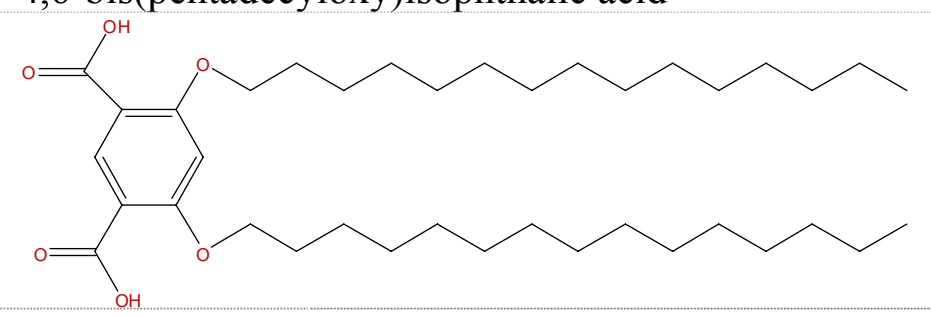

plane group $\quad p 2$

crystal class oblique

Z 2

$Z^{\prime} \quad 1$

molecular $\quad \mathrm{C}(2 \mathrm{v})$

symmetry

site symmetry 1

molecular $\quad 618.93$

weight (Da)

multicomponent no

De Feyter, S., Gesquière, A., Klapper, M., Müllen, K.

\& De Schryver, F. C. Nano Lett. 2003 3, 1485-1488.

Record 164

5-(tricosan-12-yloxy)isophthalic acid compound with octanol

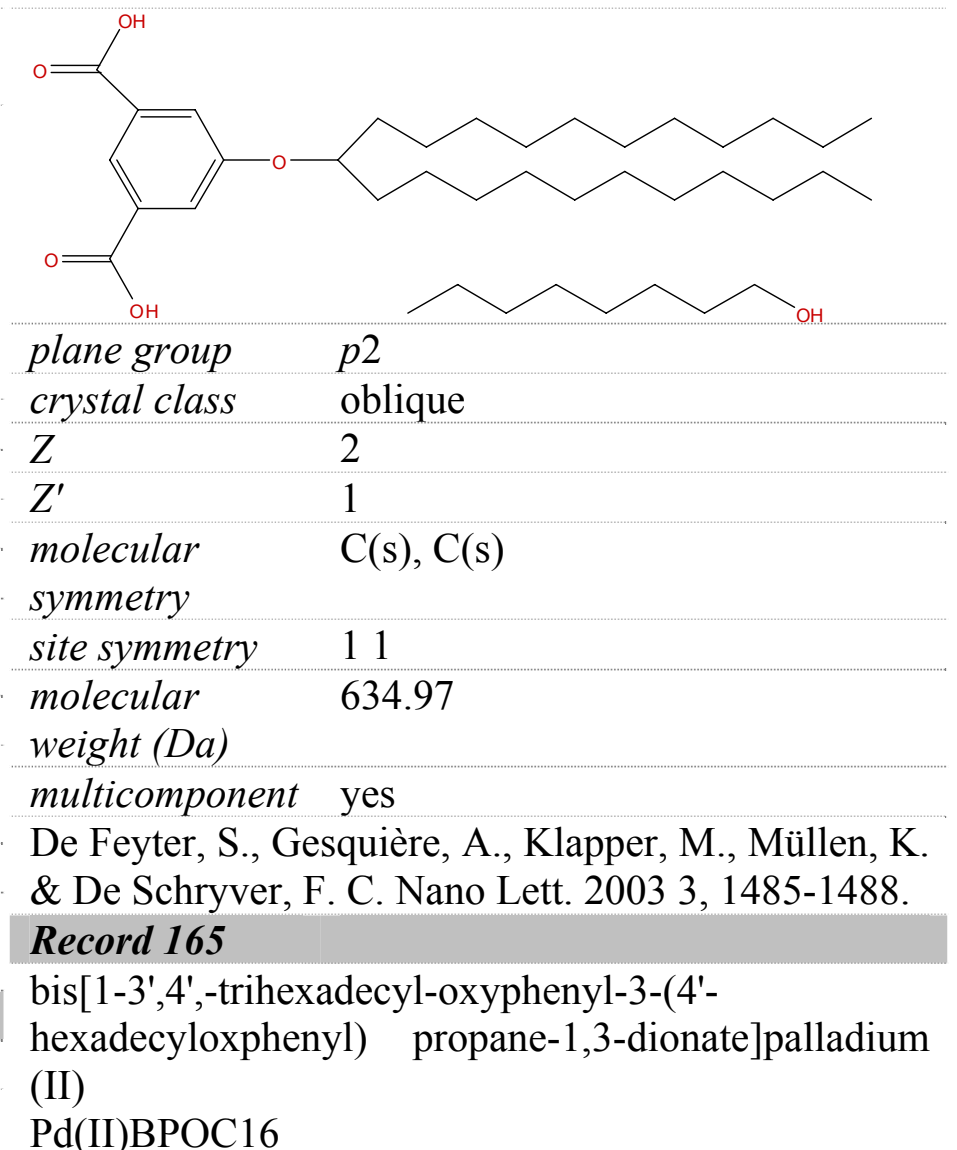

\section{Pd(II)BPOC16}

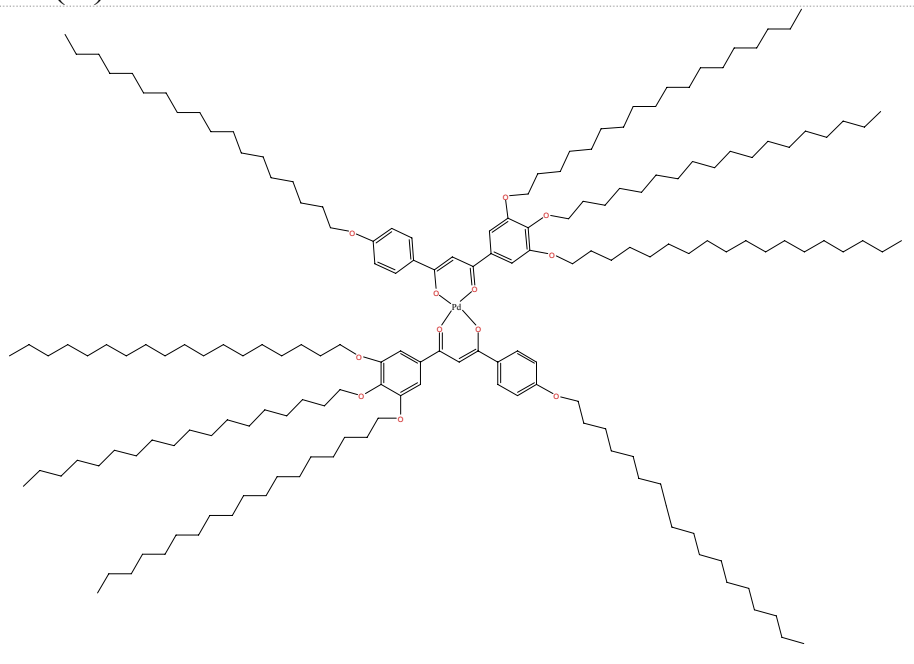

plane group $\quad p 2$

crystal class oblique

$\begin{array}{ll}Z & 1 \\ Z^{\prime} & 0.5 \\ \text { molecular } & \mathrm{C}(2 \mathrm{~h}) \\ \text { symmetry } & \\ \text { site symmetry } & 2 \\ \text { molecular } & 2700.74 \\ \text { weight (Da) } & \\ \text { multicomponent } & \text { no }\end{array}$


Su, C., Yu, S. H., Shu, C. R., Wang, H. H. \& Lai, C.

K. Synth. Met. 2003 137, 915-916.

\section{Record 166}

bis(4-dibutylamino-2-phenyl)squaraine

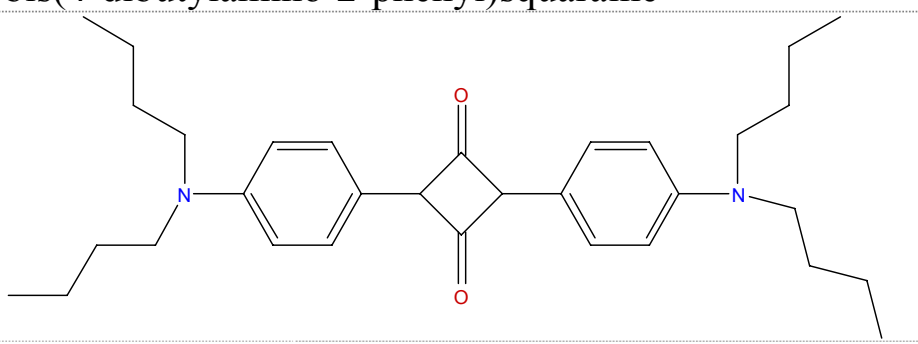

plane group $\quad$ 2

crystal class oblique

$\begin{array}{ll}Z & 1 \\ Z^{\prime} & 0.5\end{array}$

molecular $\mathrm{C}(2 \mathrm{v})$

symmetry

weight $(\mathrm{Da})$

site symmetry 2

molecular $\quad 490.72$

weight (Da)

multicomponent no

Yasuda, S. \& Shigekawa, H. Jpn. J. Appl. Phys., Part 12003 42, 4901-4904.

\section{Record 167}

dodecyl-substituted quaterthiophene

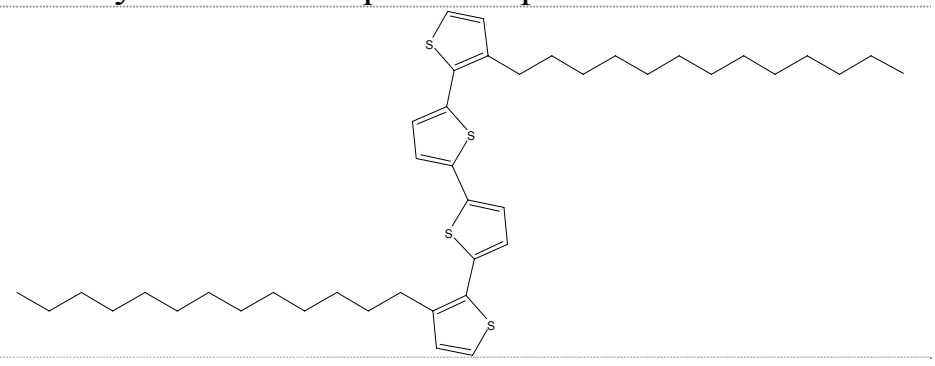

plane group $\quad$ p2

crystal class oblique

Z 1

$Z^{\prime} \quad 0.5$

molecular $\mathrm{C}(2 \mathrm{~h})$

symmetry

site symmetry 2

molecular

695.20

weight (Da)

multicomponent no

Mena-Osteritz, E. Adv. Mater. 2002 14, 609-616.

\section{Record 168}

Por-2H

\begin{tabular}{ll} 
plane group & $p 2$ \\
crystal class & oblique \\
\hline$Z$ & 1 \\
\hline$Z^{\prime}$ & 0.5 \\
$\begin{array}{l}\text { molecular } \\
\text { symmetry }\end{array}$ & $\mathrm{C}(2 \mathrm{~h})$ \\
site symmetry & 2 \\
$\begin{array}{l}\text { molecular } \\
\text { weight }(\mathrm{Da})\end{array}$ & 999.50 \\
\hline
\end{tabular}

multicomponent no

Ohshiro, T., Ito, T., Bühlmann, P. \& Umezawa, Y.

Anal. Chem. 2001 73, 878-883.

\section{Record 169}

16-hydroxyhexadecanoic acid

$\begin{array}{ll}\text { plane group } & p 2 \\ \text { crystal class } & \text { oblique } \\ Z & 2 \\ Z \prime & 1 \\ \begin{array}{l}\text { molecular } \\ \text { symmetry }\end{array} & \mathrm{C}(\mathrm{s}) \\ \text { site symmetry } & 11\end{array}$

molecular $\quad 272.42$

weight (Da)

multicomponent no

Wintgens, D., Yablon, D. G. \& Flynn, G. W. J. Phys.

Chem. B 2003 107, 173-179.

\section{Record 170}

4-n-pentyloxy-4'-cyanobiphenyl (5OCB)

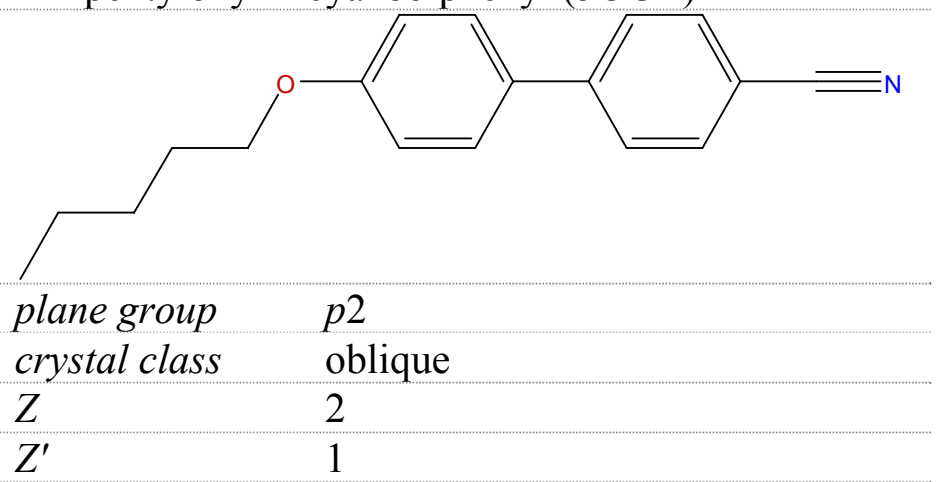


molecular

$\mathrm{C}(\mathrm{s})$

symmetry

site symmetry 1

molecular 265.35

weight (Da)

multicomponent no

Walzer, K. \& Hietschold, M. J. Vac. Sci. Technol., B 1996 14, 1461-1465.

\section{Record 171}

1,3,8,10-tetramethyl-N,N'-didocosyl-quinacridone

TmQA-C22

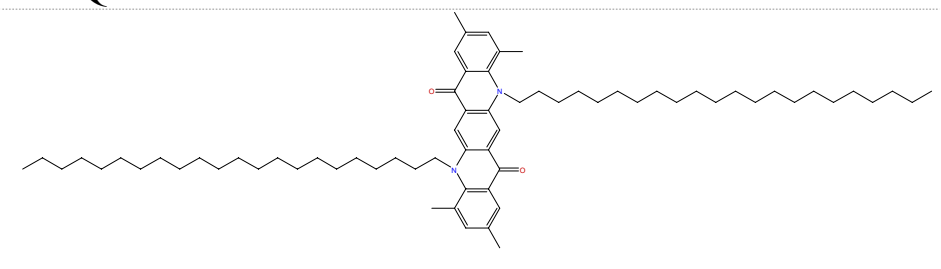

plane group $\quad p 2$

crystal class oblique

$Z \quad 1$

$Z^{\prime} \quad 0.5$

molecular $\mathrm{C}(2 \mathrm{~h})$

symmetry

site symmetry 2

molecular $\quad 985.60$

weight (Da)

multicomponent no

Mu, Z., Wang, Z., Zhang, X., Ye, K. \& Wang, Y. J.

Phys. Chem. B 2004 108, 19955-19959.

\section{Record 172}

N,N'-didocosyl-quinacridone

QA-C22

\begin{tabular}{ll} 
plane group & oblique \\
crystal class & obliqu \\
$Z$ & 1 \\
\hline$Z^{\prime}$ & 0.5 \\
molecular & $\mathrm{C}(2 \mathrm{~h})$ \\
symmetry & 2 \\
site symmetry & 2 \\
molecular & 929.49 \\
weight (Da) & \\
multicomponent & no
\end{tabular}

Mu, Z., Wang, Z., Zhang, X., Ye, K. \& Wang, Y. J. Phys. Chem. B 2004 108, 19955-19959.

\section{Record 173}

$\mathrm{N}, \mathrm{N}$ '-didocosyl-quinacridone (QA-C22) and steric acid

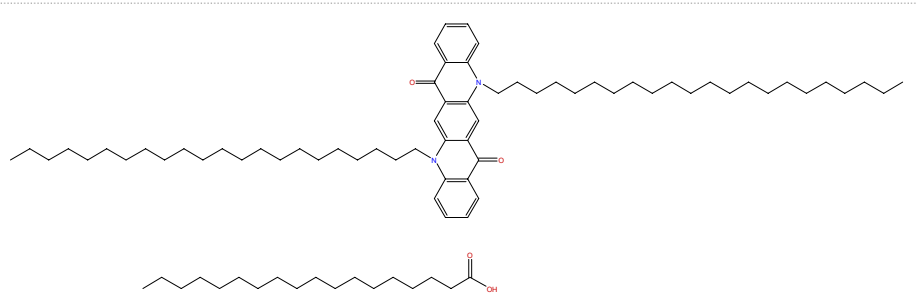

$$
\begin{array}{ll}
\text { plane group } & p 2 \\
\text { crystal class } & \text { oblique } \\
Z Z & 2 \\
Z^{\prime} & 1 \\
\text { molecular } & \mathrm{C}(\mathrm{s}), \mathrm{C}(2 \mathrm{~h})
\end{array}
$$

symmetry

site symmetry 12

molecular $\quad 1213.97$

weight (Da)

multicomponent yes

Mu, Z., Wang, Z., Zhang, X., Ye, K. \& Wang, Y. J.

Phys. Chem. B 2004 108, 19955-19959.

\section{Record 174}

1,3,8,10-tetramethyl-N,N'-dihexadecyl-quinacridone (TmQA-C16) and palmitic acid

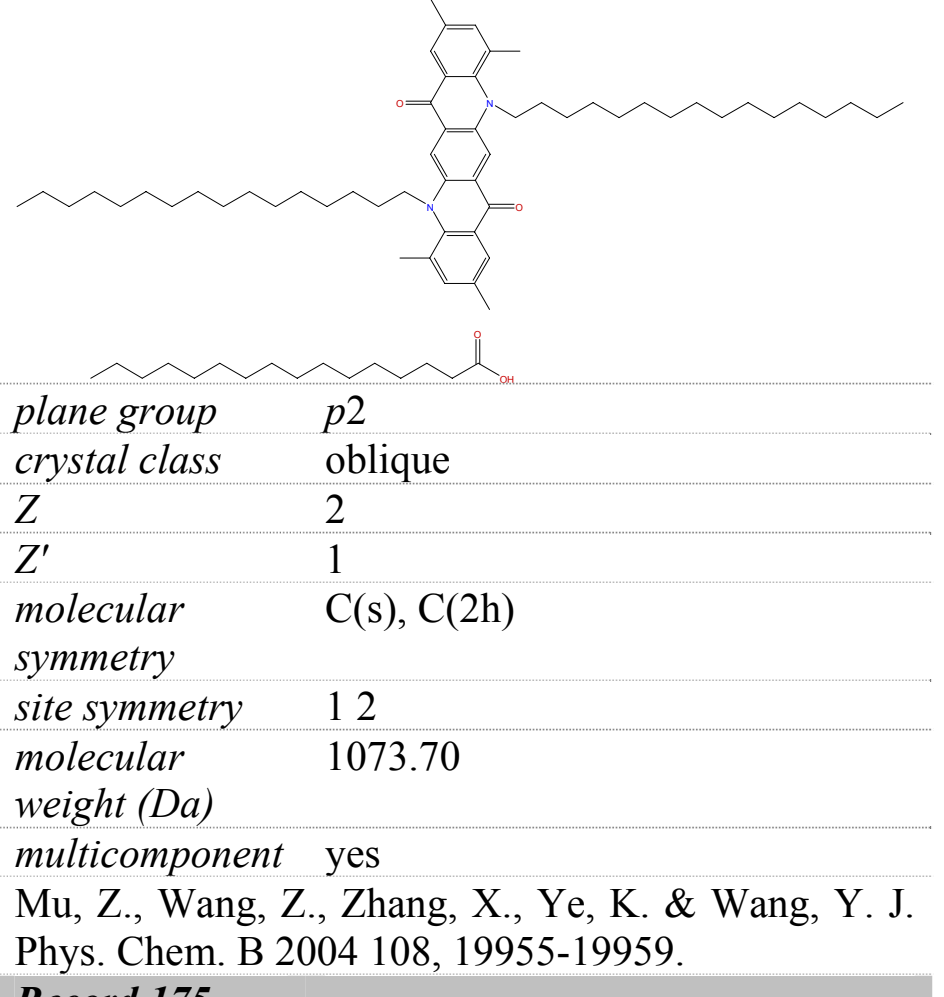


1-(4-amino-6-(4-(4-(2,5-bis((S)-2-methylbutoxy)-4(3,4,5-tris(dodecyloxy)styryl)styryl)-2,5-bis((S)-2methylbutoxy)styryl)phenyl)-1,3,5-triazin-2-yl)-3butylurea

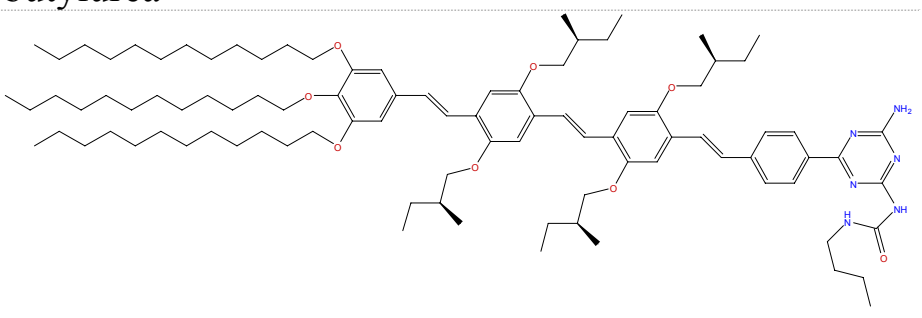

\begin{tabular}{|c|c|}
\hline plane group & $p 2$ \\
\hline crystal class & oblique \\
\hline$Z$ & 2 \\
\hline$Z^{\prime}$ & 1 \\
\hline $\begin{array}{l}\text { molecular } \\
\text { symmetry }\end{array}$ & $\mathrm{C}(1)$ \\
\hline site symmetry & 1 \\
\hline $\begin{array}{l}\text { molecular } \\
\text { weight }(\mathrm{Da})\end{array}$ & 1490.22 \\
\hline
\end{tabular}

Gesquière, A., Jonkheijm, P., Hoeben, F. J. M., Schenning, A. P. H. J., De Feyter, S., De Schryver, F. C. \& Meijer, E. W. Nano Lett. 2004 4, 1175-1179.

\section{Record 176}

1-(4-amino-6-(4-(4-(4-(2,5-bis((S)-2-methylbutoxy)4-(3,4,5-tris(dodecyloxy)styryl)styryl)-2,5-bis((S)-2methylbutoxy)styryl)-2,5-bis((S)-2methylbutoxy)styryl)phenyl)-1,3,5-triazin-2-yl)-3butylurea

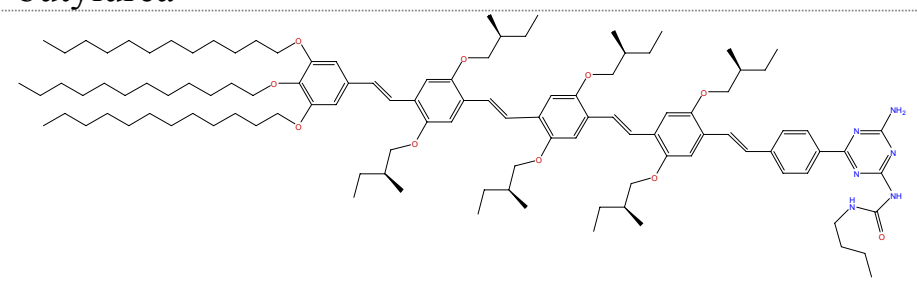

Gesquière, A., Jonkheijm, P., Hoeben, F. J. M., Schenning, A. P. H. J., De Feyter, S., De Schryver, F. C. \& Meijer, E. W. Nano Lett. 2004 4, 1175-1179.

\section{Record 177}

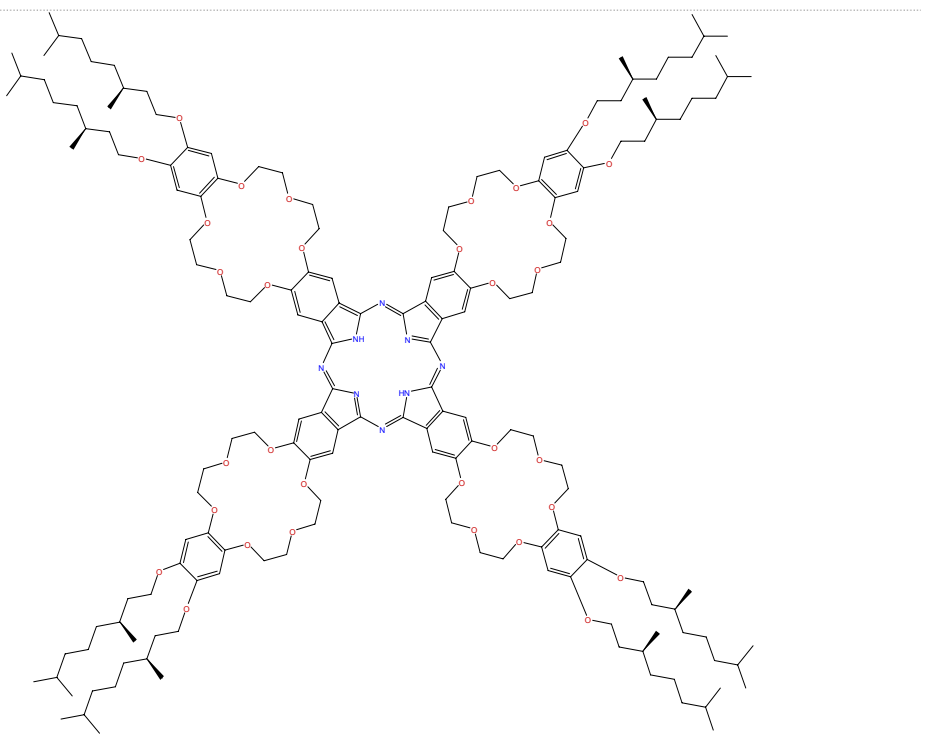

plane group $\quad p 2$

crystal class oblique

Z 1

$Z^{\prime} \quad 0.5$

molecular $\quad \mathrm{C}(4)$

symmetry

site symmetry 2

molecular $\quad 2893.82$

weight (Da)

multicomponent no

Samorí, P., Engelkamp, H., de Witte, P., Rowan, A. E., nolte, R. J. M. \& Rabe, J. P. Angew. Chem., Int.

Edit. 2001 40, 2348-2350.

\section{Record 178}

4'-n-hexyl-4-cyanobiphenyl (6CB)

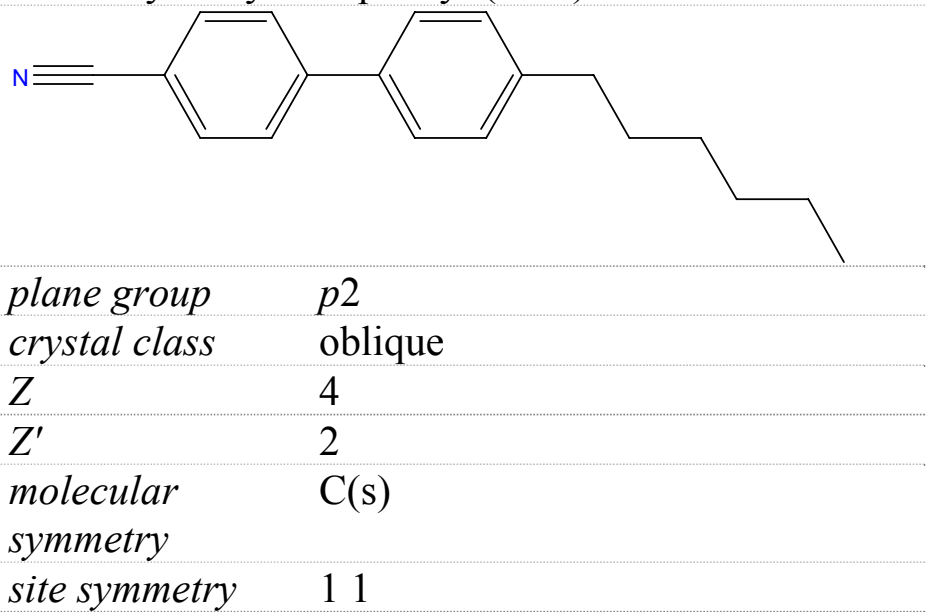


molecular

263.38

weight (Da)

multicomponent no

Smith, D. P. E., Heckl, W. M. \& Klagges, H. A. Surf.

Sci. 1992 278, 166-174.

\section{Record 179}

bis(2-(trimethylsilyl)ethyl) 4,4'-(4,4'-(buta-1,3-diyne1,4-diyl)bis(2,5-bis(dodecyloxy)-4,1-

phenylene))bis(ethyne-2,1-diyl)dibenzoate

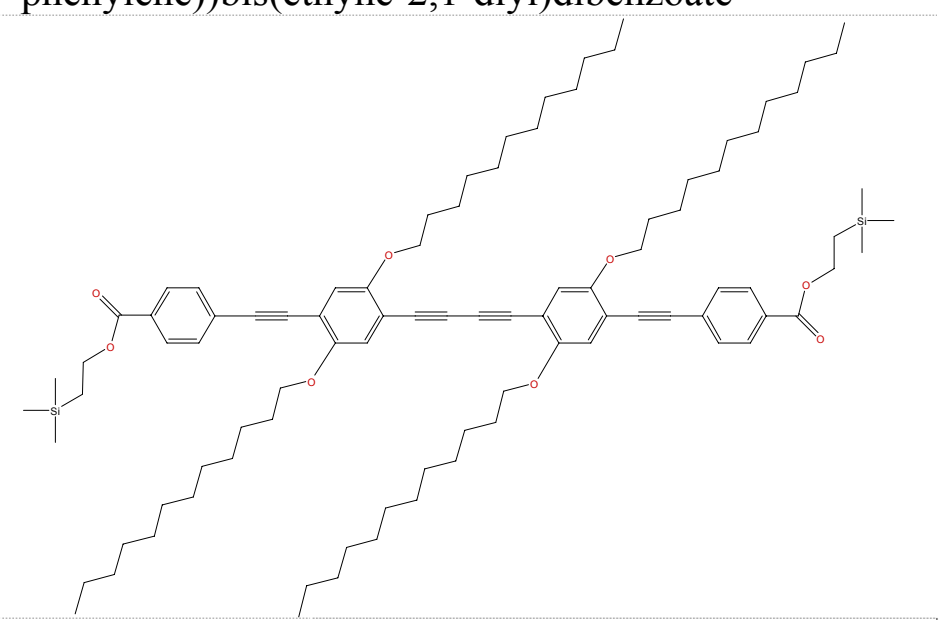

plane group p2

crystal class oblique

$\begin{array}{ll}Z & 1 \\ Z^{\prime} & 0.5\end{array}$

molecular $\quad \mathrm{C}(2 \mathrm{~h})$

symmetry

site symmetry 2

molecular

1428.25

weight (Da)

multicomponent no

Mu, Z., Yang, X., Wang, Z., Zhang, X., Zhao, J. \& Bo, Z. Langmuir 2004 20, 8892-8896.

\section{Record 180}

2,3,6,7,10,11-hexakis(hexylthio)triphenylene

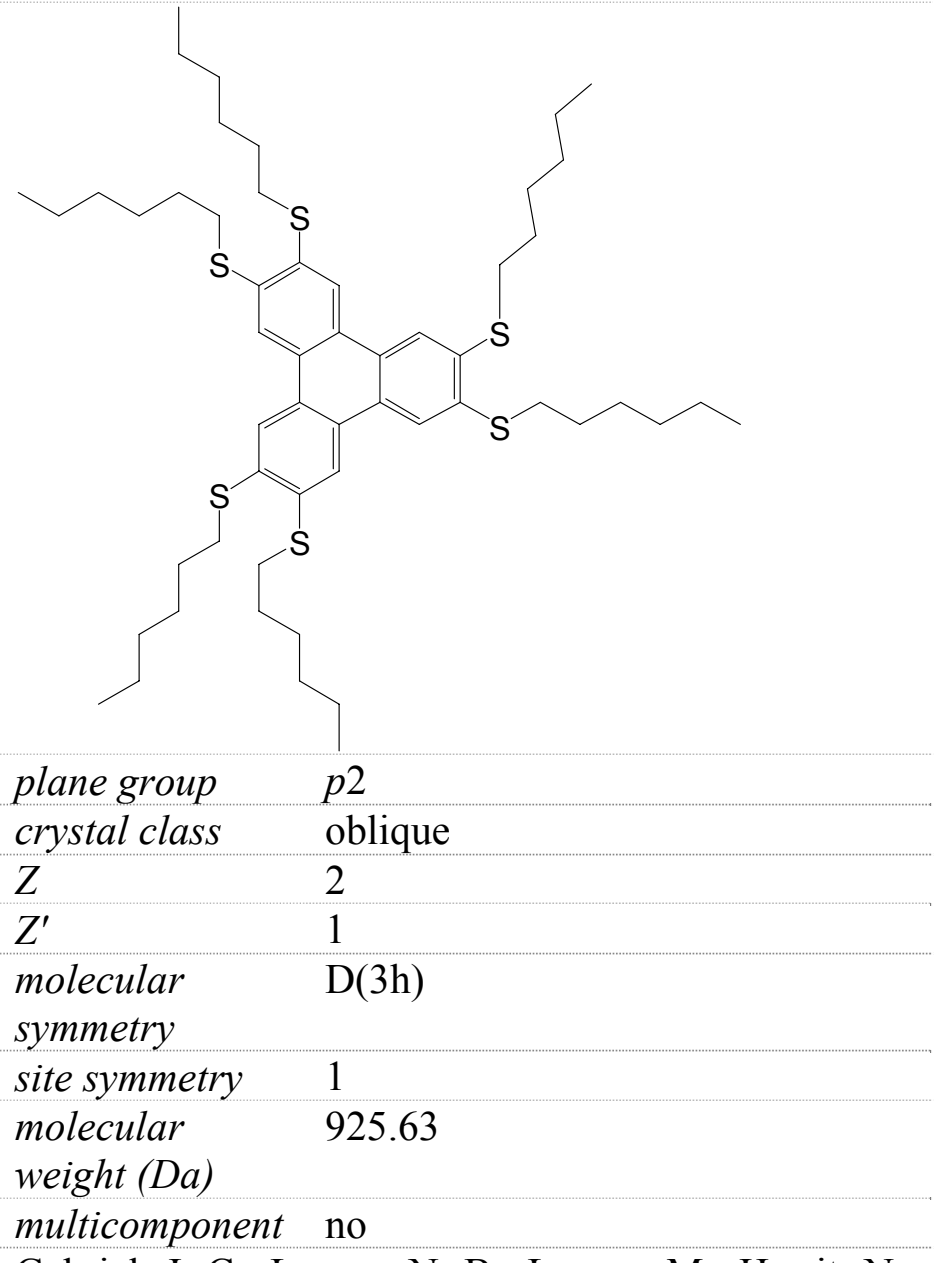

Gabriel, J. C., Larsen, N. B., Larsen, M., Harrit, N., Pedersen, J. S., Schaumburg, K. \& Bechgaard, K. Langmuir 1996 12, 1690-1692.

\section{Record 181}

4,4'-bis(decyloxy)biphenyl (4)

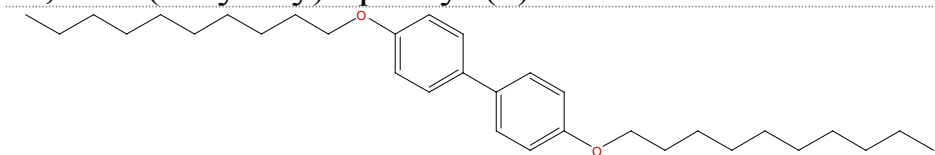

\begin{tabular}{|c|c|}
\hline plane group & $p 2$ \\
\hline crystal class & oblique \\
\hline$Z$ & 2 \\
\hline$Z^{\prime}$ & 1 \\
\hline $\begin{array}{l}\text { molecular } \\
\text { symmetry }\end{array}$ & $\mathrm{C}(2 \mathrm{v})$ \\
\hline site symmetry & 1 \\
\hline $\begin{array}{l}\text { molecular } \\
\text { weight }(D a)\end{array}$ & 466.74 \\
\hline multicomponent & no \\
\hline $\begin{array}{l}\text { Hansen, T., Itoua } \\
\text { J. B., Bjørnholm, } \\
\text { \& Wilkes, S. B. J }\end{array}$ & $\begin{array}{l}\text { S., Kamounah, F. S., Christensen, } \\
\text { T., Schaumburg, K., Bechgaard, K. } \\
\text { Mater. Chem. 1999 9, 1107-1113. }\end{array}$ \\
\hline
\end{tabular}




\section{Record 182}

4,4'-bis(hexadecyloxy)biphenyl (5)

plane group p2

crystal class oblique

$Z \quad 1$

$Z^{\prime} \quad 0.5$

molecular $\mathrm{C}(2 \mathrm{v})$

symmetry

site symmetry 2

molecular 635.06

weight (Da)

multicomponent no

Hansen, T., Itoua, S., Kamounah, F. S., Christensen, J. B., Bjørnholm, T., Schaumburg, K., Bechgaard, K. \& Wilkes, S. B. J. Mater. Chem. 1999 9, 1107-1113.

\section{Record 183}

5-(dimethylamino)-5"'-nitro-2,2':5',2"-terthiophene (2a)

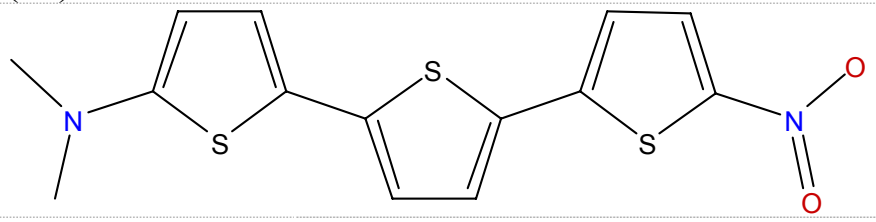

plane group $\quad$ 2

crystal class oblique

$Z \quad 2$

$Z^{\prime} \quad 1$

molecular $\quad \mathrm{C}(\mathrm{s})$

symmetry

site symmetry 1

molecular

337.46

weight (Da)

multicomponent no

Stecher, R., Drewnick, F. \& Gompf, B. Langmuir 1999 15, 6490-6494.

\section{Record 184}

5-(dimethylamino)-5"-cyanovinyl-2,2':5',2"terthiophene (2b)

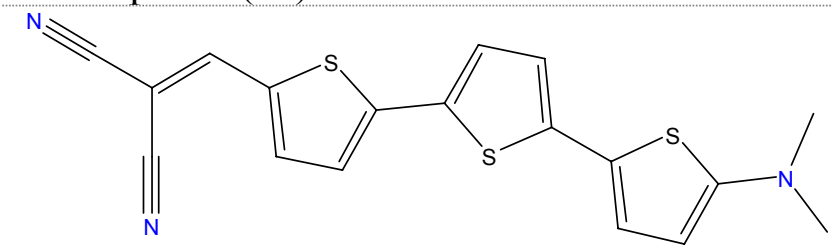

plane group $\quad p 2$

crystal class oblique

$\begin{array}{ll}Z & 2 \\ Z^{\prime} & 1 \\ \text { molecular } & \mathrm{C}(\mathrm{s}) \\ \text { symmetry } & \\ \text { site symmetry } & 1 \\ \begin{array}{l}\text { molecular } \\ \text { weight }(\mathrm{Da})\end{array} & 367.51 \\ \text { multicomponent } & \text { no }\end{array}$

Stecher, R., Drewnick, F. \& Gompf, B. Langmuir 1999 15, 6490-6494.

\section{Record 185}

R-2-bromohexadecanoic acid

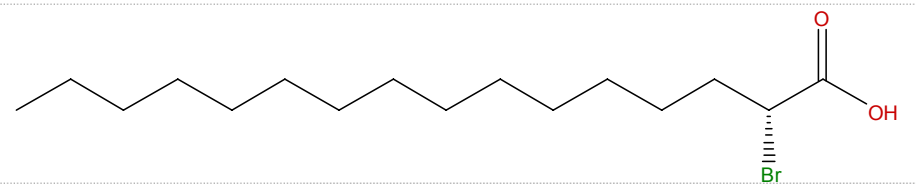

plane group $\quad p 2$

crystal class oblique

Z 2

$Z^{\prime} \quad 1$

molecular $\quad \mathrm{C}(1)$

symmetry

site symmetry 1

molecular $\quad 335.32$

weight (Da)

multicomponent no

Fang, H. B., Giancarlo, L. C. \& Flynn, G. W. J. Phys.

Chem. B 1998 102, 7311-7315.

\section{Record 186}

1-(4-amino-6-(4-(2,5-bis((S)-2-methylbutoxy)-4-

(3,4,5-tris(dodecyloxy)styryl)styryl)phenyl)-1,3,5-

triazin-2-yl)-3-butylurea

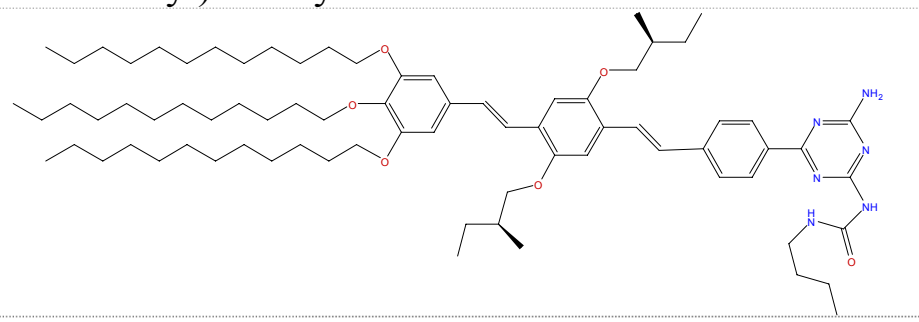

plane group $\quad$ p2

crystal class oblique

$Z$
$Z$

$Z^{\prime} \quad 1$

molecular $\quad \mathrm{C}(1)$

symmetry

site symmetry 1

molecular $\quad 1215.82$

weight (Da) 
multicomponent no

Gesquière, A., Jonkheijm, P., Hoeben, F. J. M., Schenning, A. P. H. J., De Feyter, S., De Schryver, F. C. \& Meijer, E. W. Nano Lett. 2004 4, 1175-1179.

\section{Record 187}

Zinc(II) 2,3,9,10,16,17,23,24-octakis(octyloxy)$29 \mathrm{H}, 31 \mathrm{H}$ - phthalocyanine

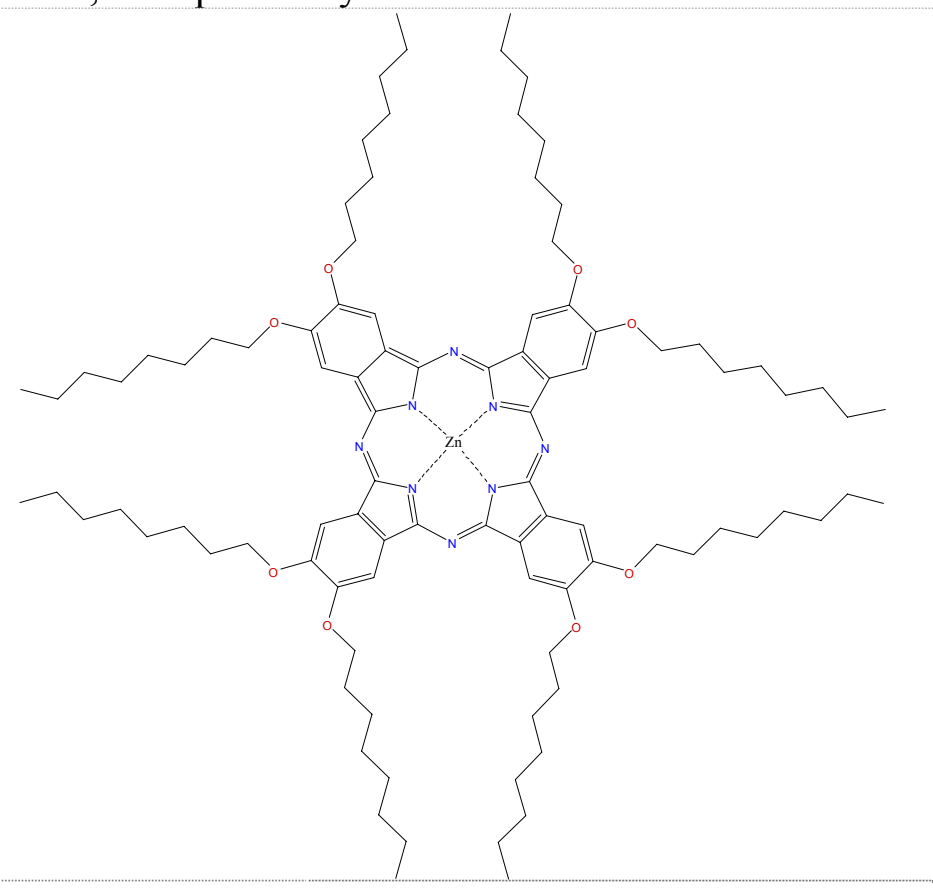

plane group p2

crystal class oblique

$\begin{array}{ll}Z & 1 \\ Z^{\prime} & 0.5\end{array}$

molecular $\mathrm{D}(4 \mathrm{~h})$

symmetry

site symmetry 2

molecular $\quad 1603.62$

weight (Da)

multicomponent no

Qiu, X. H., Wang, C., Yin, S. X., Zeng, Q. D., Xu, B. \& Bai, C. L. J. Phys. Chem. B 2000 104, 3570-3574.

\section{Record 188}

11-Bromoundecanoic acid, $\mathrm{Br}(\mathrm{CH} 2) 10 \mathrm{COOH}$

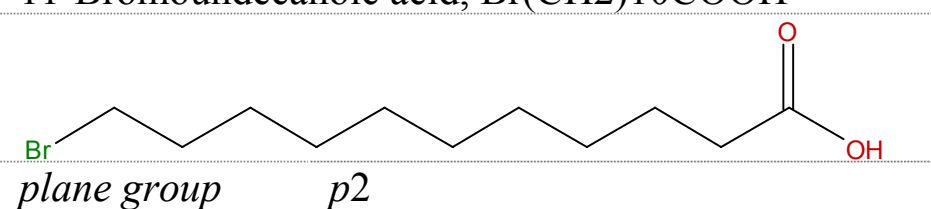

$\begin{array}{ll}\text { crystal class } & \text { oblique } \\ Z & 2 \\ Z^{\prime} & 1 \\ \text { molecular } & \mathrm{C}(\mathrm{s})\end{array}$

symmetry site symmetry 1

molecular 265.19

weight (Da)

multicomponent no

Fang, H. B., Giancarlo, L. C. \& Flynn, G. W. J. Phys.

Chem. B 1998 102, 7421-7424.

\section{Record 189}

octadecanamide (aka stearoyl amide)

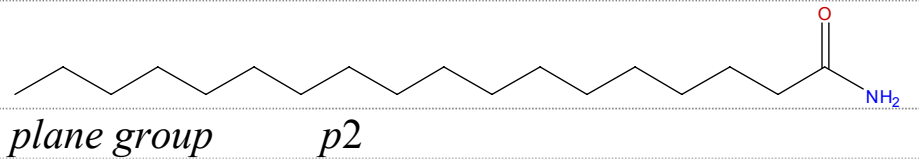

crystal class oblique

$Z \quad 2$

$Z^{\prime} \quad 1$

molecular $\mathrm{C}(\mathrm{s})$

symmetry

site symmetry 1

molecular $\quad 283.49$

weight (Da)

multicomponent no

Giancarlo, L., Cyr, D., Muyskens, K. \& Flynn, G. W.

Langmuir 1998 14, 1465-1471.

\section{Record 190}

a-phenylethynyl-w-phenyl-

ter[(2,5-dihexylphenylene-1,4)ethynylene)] (9)

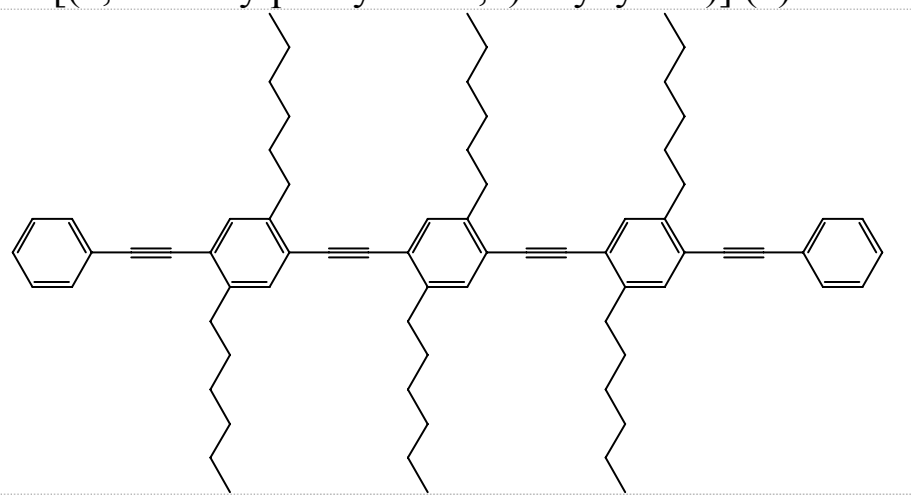

plane group $\quad p 2$

crystal class oblique

Z 1

$Z^{\prime} \quad 0.5$

molecular $\quad \mathrm{C}(2 \mathrm{~h})$

symmetry

site symmetry 2

molecular $\quad 983.54$

weight (Da)

multicomponent no

Samorí, P., Francke, V., Müllen, K. \& Rabe, J. P.

Chem.-Eur. J. 1999 5, 2312-2317. 


\section{Record 191}

dibenzo-18-crown-6

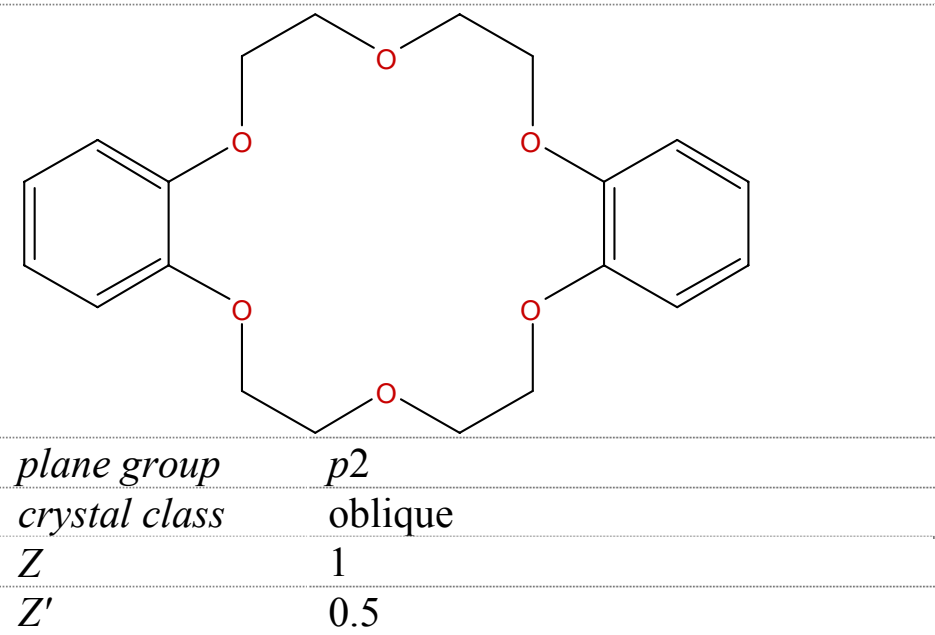

molecular $\mathrm{D}(2 \mathrm{~h})$

symmetry

site symmetry 2

molecular $\quad 360.40$

weight (Da)

multicomponent no

Kuwahara, Y., Saito, A. \& Aono, M. Seisan to Gijutsu 1999 51, 55-58.

\section{Record 192}

2-bromohexadecanoic acid

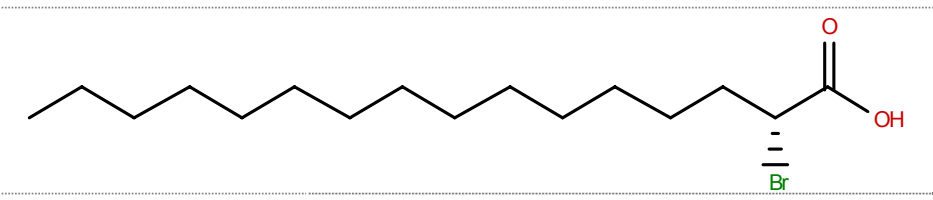

plane group $\quad p 2$

crystal class oblique

$\begin{array}{ll}Z & 2 \\ Z^{\prime} & 1\end{array}$

molecular $\quad \mathrm{C}(1)$

symmetry

site symmetry 1

molecular $\quad 670.64$

weight $(D a)$

multicomponent no

Fang, H. B., Giancarlo, L. C. \& Flynn, G. W. J. Phys.

Chem. B 1999 103, 5712-5715.

\section{Record 193}

4-n-octyl-4'-cyanobiphenyl (8CB)

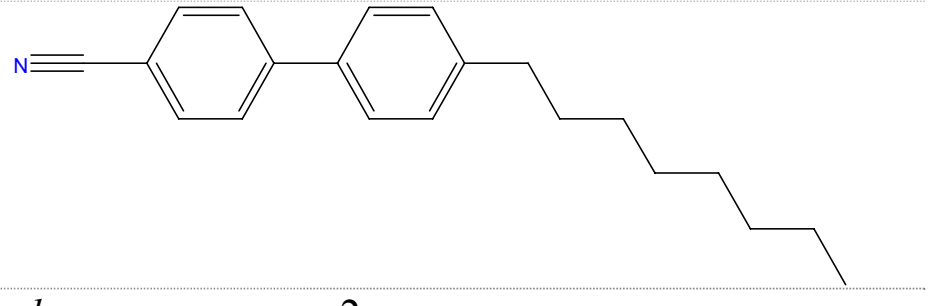

plane group $\quad p 2$

crystal class oblique

Z 8

$Z^{\prime} \quad 4$

molecular $\quad \mathrm{C}(\mathrm{s})$

symmetry

site symmetry $\quad 1111$

molecular 291.43

weight (Da)

multicomponent no

Baker, R. T., Mougous, J. D., Brackley, A. \& Patrick,

D. L. Langmuir 1999 15, 4884-4891.

\section{Record 194}

bisphenanthroline macrocycle

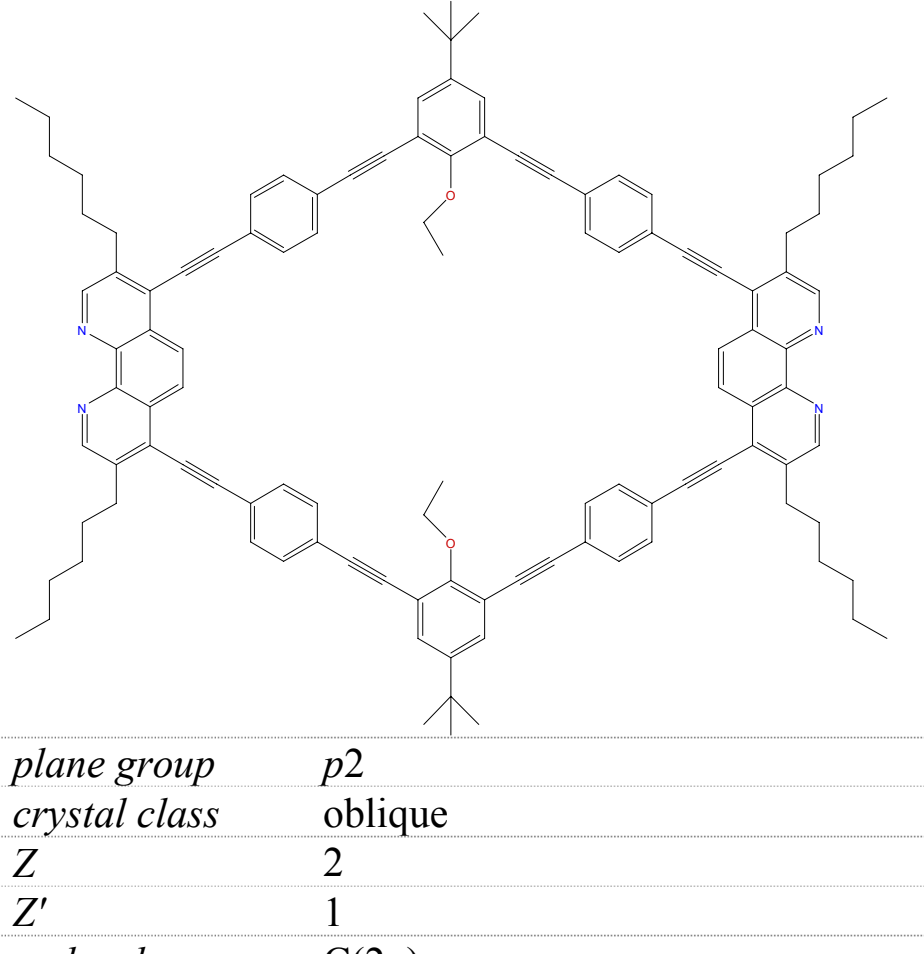

molecular $\quad \mathrm{C}(2 \mathrm{v})$

symmetry

site symmetry 1

molecular $\quad 1542.08$

weight (Da)

multicomponent no

Kalsani, V., Ammon, H., Jäckel, F., Rabe, J. P. \&

Schmittel, M. Chem.-Eur. J. 2004 10, 5481-5492. 


\section{Record 195}

cholesterol stearate

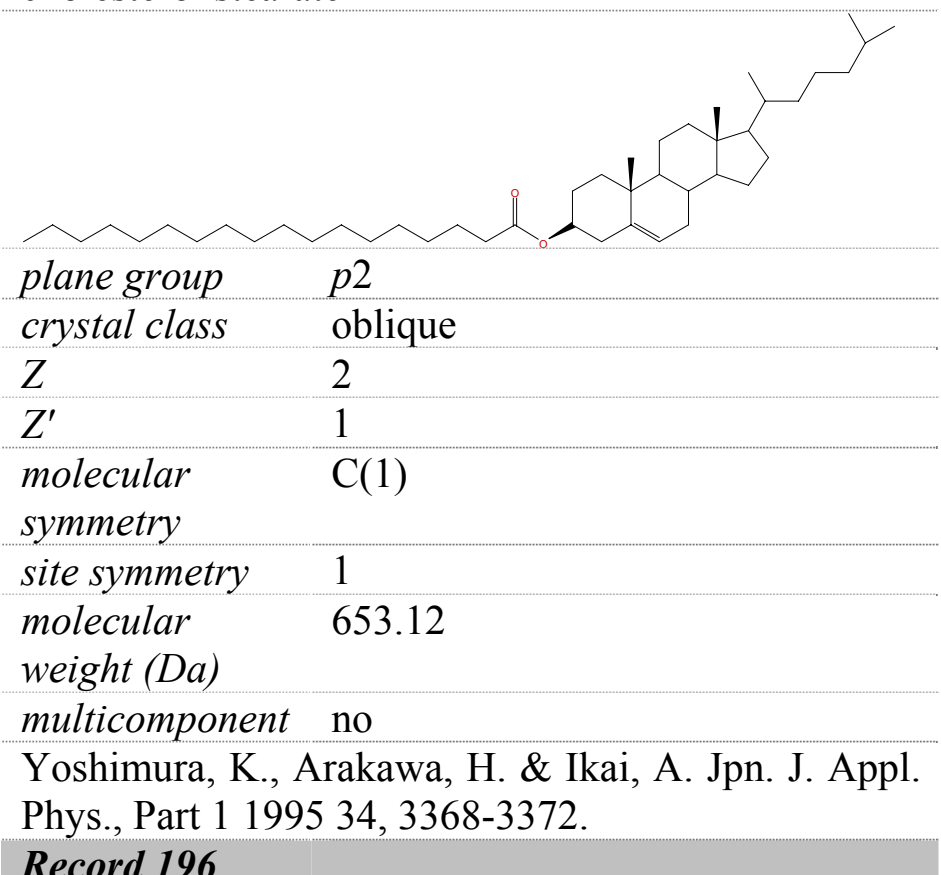

\section{Record 196}

4,4'-(4,4'-(buta-1,3-diyne-1,4-diyl)bis(2,5-

bis(dodecyloxy)-4,1-phenylene))bis(ethyne-2,1-

diyl)dibenzoic acid

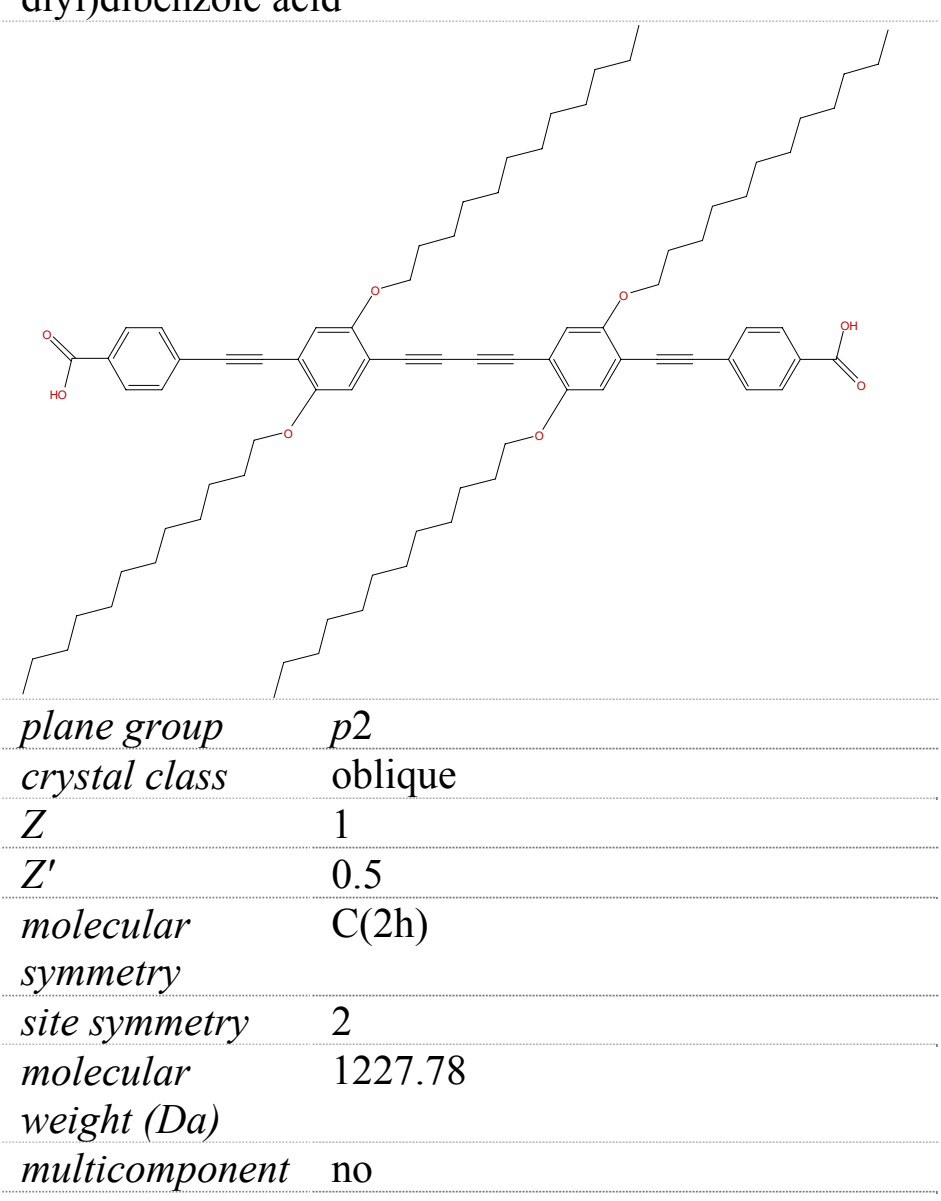

Mu, Z., Yang, X., Wang, Z., Zhang, X., Zhao, J. \& Bo, Z. Langmuir 2004 20, 8892-8896.

\section{Record 197}

cerotic acid

plane group
crystal class
$Z$

molecular $\mathrm{C}(\mathrm{s})$

symmetry

site symmetry 1

molecular 396.69

weight (Da)

multicomponent no

Yoshimura, K., Arakawa, H. \& Ikai, A. Jpn. J. Appl.

Phys., Part 11995 34, 3368-3372.

\section{Record 198}

1,11-bis-(hexadecyloxyl)undecane

$\begin{array}{ll}\text { plane group } & p 2 \\ \text { crystal class } & \text { oblique } \\ Z & 1 \\ Z^{\prime} & 0.5 \\ \text { molecular } & \mathrm{C}(2 \mathrm{v}) \\ \text { symmetry } & \\ \text { site symmetry } & 2 \\ \text { molecular } & 637.16 \\ \text { weight (Da) } & \\ \text { multicomponent } & \text { no } \\ \text { Nishino, T., Bühlmann, P., Ito, T. \& Umezawa, Y. } \\ \text { Surf. Sci. 2001 490, L579-L584. }\end{array}$

Record 199

dodecoxyl phthalocyanine 


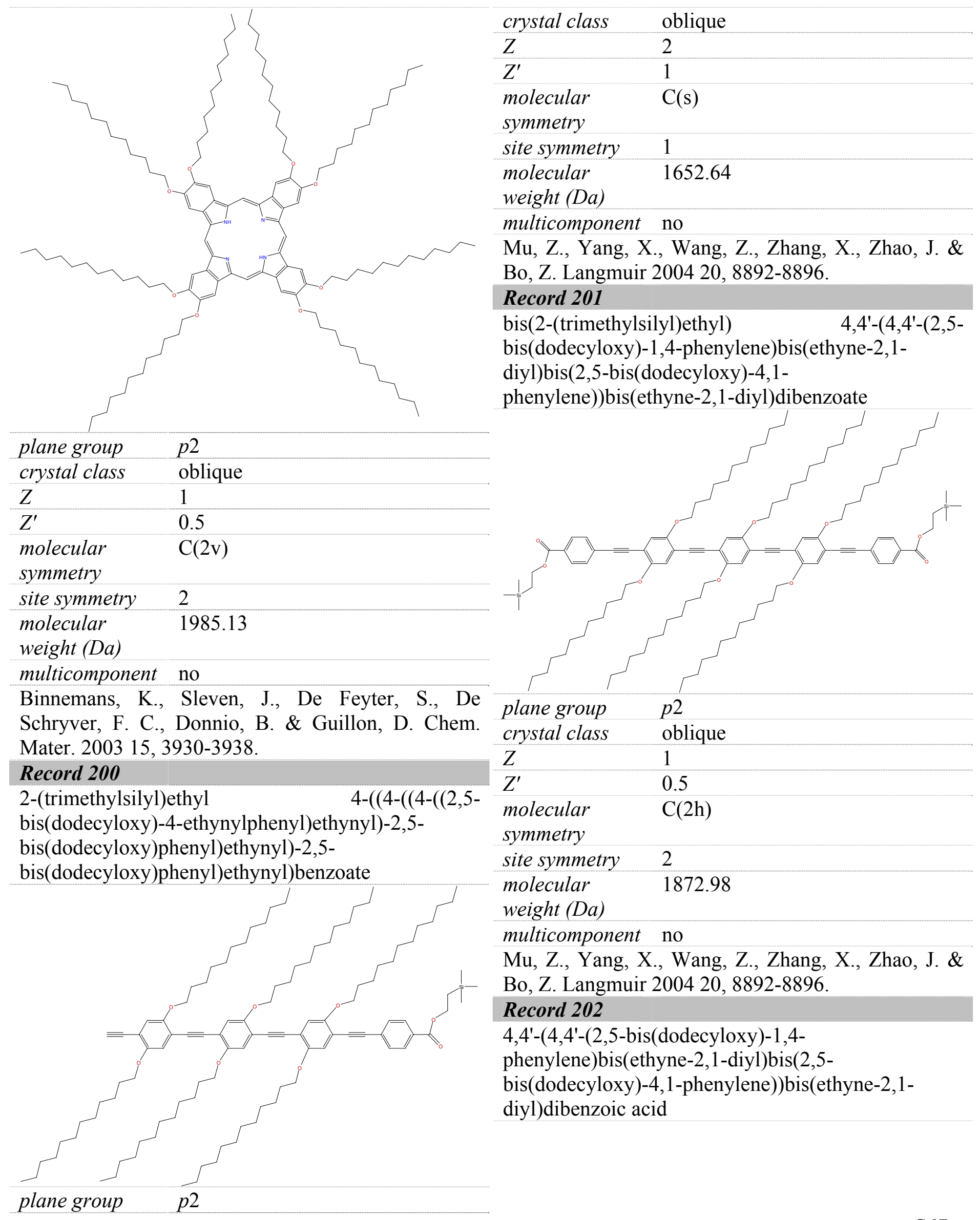




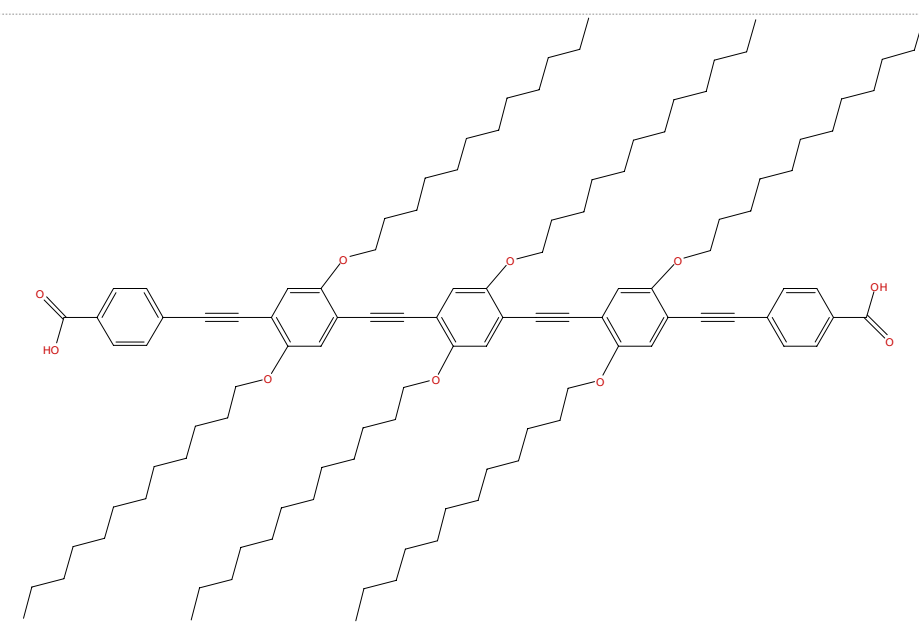

plane group p2

crystal class oblique

$Z \quad 1$

$Z^{\prime} \quad 0.5$

molecular $\mathrm{C}(2 \mathrm{~h})$

symmetry

site symmetry 2

molecular

1672.51

weight (Da)

multicomponent no

Mu, Z., Yang, X., Wang, Z., Zhang, X., Zhao, J. \& Bo, Z. Langmuir 2004 20, 8892-8896.

\section{Record 203}

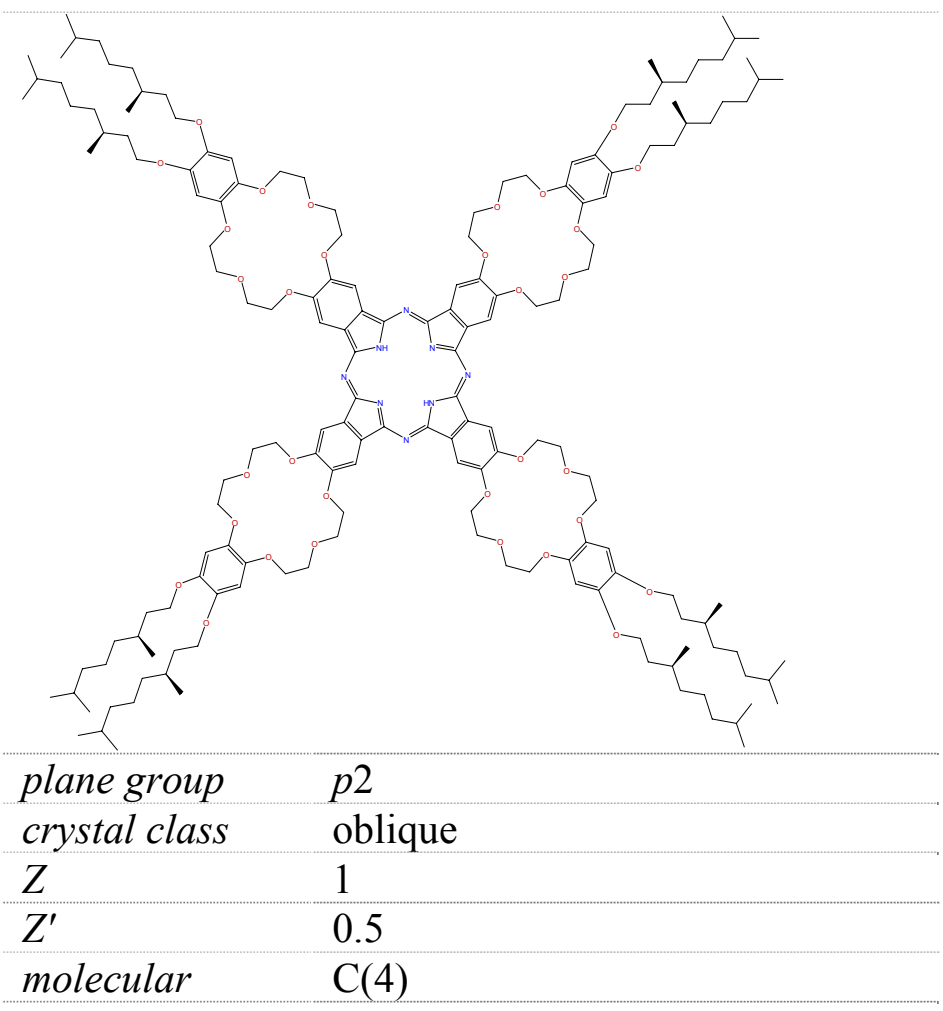

symmetry

site symmetry 2

molecular $\quad 2893.82$

weight (Da)

multicomponent no

Samorí, P., Engelkamp, H., de Witte, P., Rowan, A.

E., nolte, R. J. M. \& Rabe, J. P. Angew. Chem., Int.

Edit. 2001 40, 2348-2350.

\section{Record 204}

5-((W-perfluorohexyl)undecanyloxy)isophthalic Acid (F6H11-ISA) with octanol

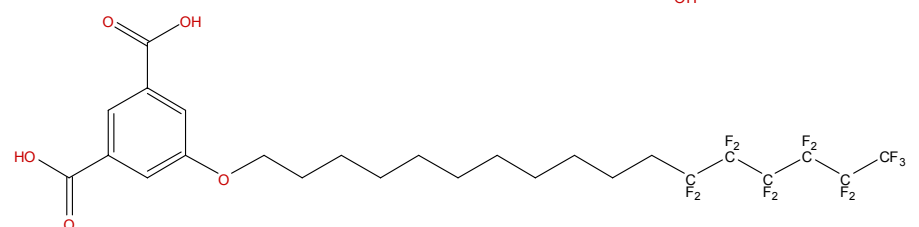

plane group $\quad p 2$

crystal class oblique

$Z \quad 2$

$Z^{\prime} \quad 1$

molecular $\quad \mathrm{C}(\mathrm{s}), \mathrm{C}(\mathrm{s})$

symmetry

site symmetry 11

molecular $\quad 784.69$

weight (Da)

multicomponent yes

Gesquière, A., Abdel-Mottaleb, M. M., De Schryver, F. C., Sieffert, M. \& Müllen, K. Langmuir 1999 15, 6821-6824.

\section{Record 205}

(R)-N-(4'-(octan-2-yloxy)biphenyl-4-yl)formamide

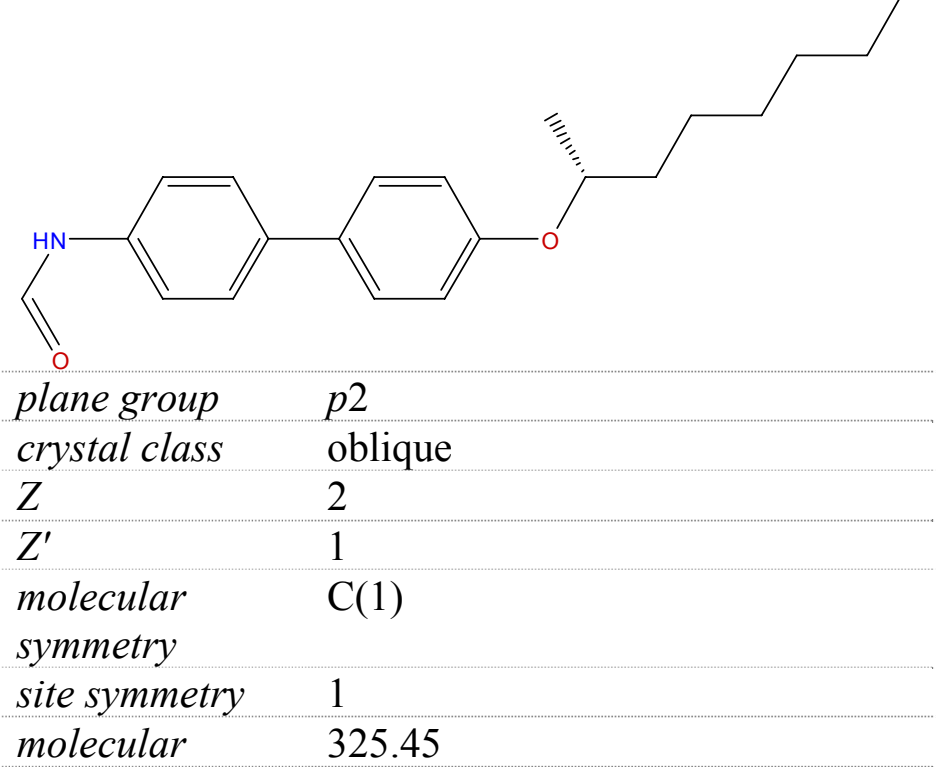


weight (Da)

multicomponent no

Mamdouh, W., Uji-i, H., Gesquière, A., De Feyter, S., Amabilino, D. B., Abdel-Mottaleb, M. M. S., Veciana, J. \& De Schryver, F. C. Langmuir 2004 20, 9628-9635.

\section{Record 206}

C18OPP

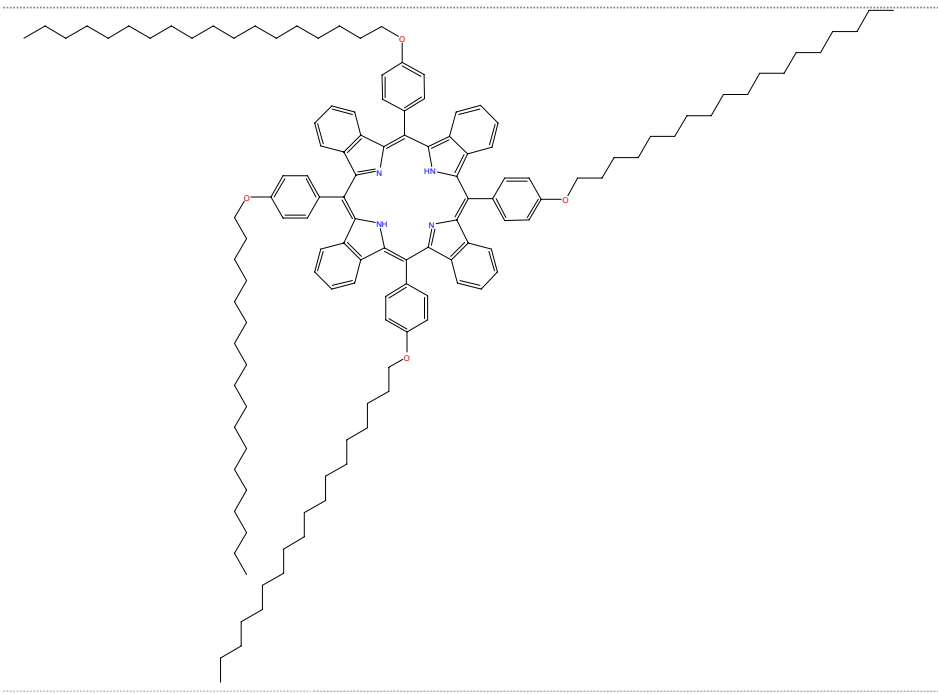

plane group p2

crystal class oblique

$Z \quad 1$

$Z^{\prime} \quad 0.5$

molecular $\mathrm{D}(2 \mathrm{~h})$

symmetry

site symmetry 2

molecular $\quad 1888.88$

weight (Da)

multicomponent no

Ikeda, T., Asakawa, M., Goto, M., Miyake, K., Ishida, T. \& Shimizu, T. Langmuir 2004 20, 54545459.

\section{Record 207}

C30OPP

\begin{tabular}{ll} 
plane group & $p 2$ \\
crystal class & oblique \\
\hline$Z$ & 1 \\
\hline$Z^{\prime}$ & 0.5 \\
$\begin{array}{l}\text { molecular } \\
\text { symmetry }\end{array}$ & $\mathrm{D}(2 \mathrm{~h})$ \\
site symmetry & 2 \\
$\begin{array}{l}\text { molecular } \\
\text { weight }(\mathrm{Da})\end{array}$ & 2562.16 \\
\hline
\end{tabular}

multicomponent no

Ikeda, T., Asakawa, M., Goto, M., Miyake, K., Ishida, T. \& Shimizu, T. Langmuir 2004 20, 54545459.

\section{Record 208 \\ $\mathrm{Rh}(\mathrm{C} 30 \mathrm{OPP}) \mathrm{Cl}$}

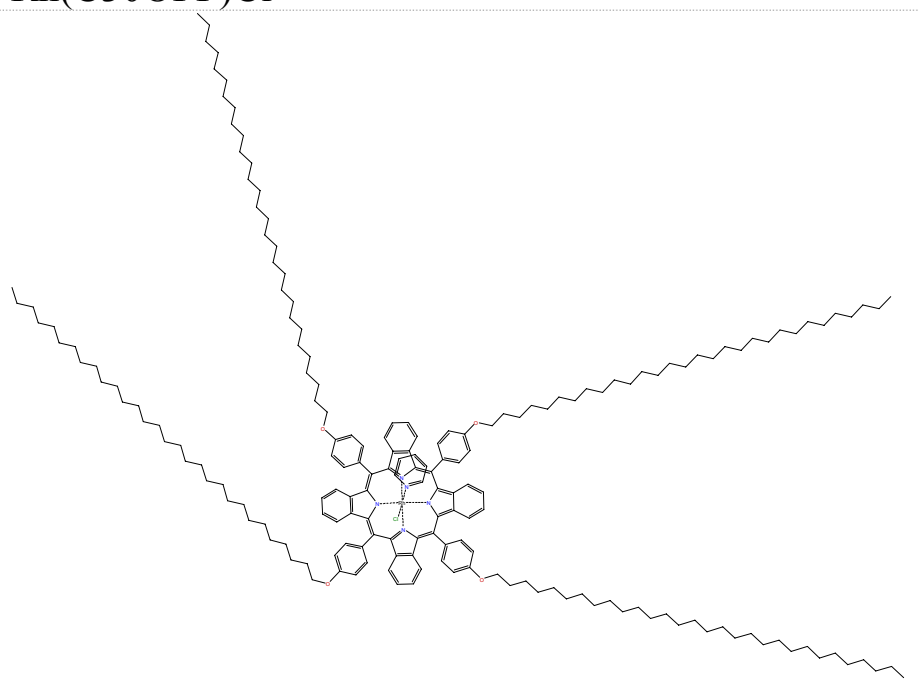

\begin{tabular}{ll} 
plane group & $p 2$ \\
crystal class & oblique \\
$Z$ & 1 \\
\hline$Z^{\prime}$ & 0.5
\end{tabular}


molecular

symmetry

site symmetry

molecular

2

weight (Da)

multicomponent no

Ikeda, T., Asakawa, M., Goto, M., Miyake, K., Ishida, T. \& Shimizu, T. Langmuir 2004 20, 54545459.

\section{Record 209}

terephthalic acid<smiles>O=C(O)c1ccc(C(=O)O)cc1</smiles>

plane group $\quad p 2$

crystal class oblique

$\begin{array}{ll}Z & 1 \\ Z^{\prime} & 0.5\end{array}$

molecular $\mathrm{C}(2 \mathrm{v})$

symmetry

site symmetry 2

molecular $\quad 166.13$

weight (Da)

multicomponent no

Lackinger, M., Griessl, S., Markert, T., Jamitzky, F. \& Heckl, W. M. J. Phys. Chem. B 2004 108, 1365213655.

\section{Record 210}

isophthalic acid<smiles>O=C(O)c1cccc(C(=O)O)c1</smiles>

plane group $\quad p 2$

crystal class oblique

$Z-2$

$Z^{\prime} \quad 1$

molecular $\quad \mathrm{C}(2 \mathrm{v})$

symmetry

site symmetry 1

molecular

166.13 weight (Da)

multicomponent no

Lackinger, M., Griessl, S., Markert, T., Jamitzky, F. \& Heckl, W. M. J. Phys. Chem. B 2004 108, 1365213655.

\section{Record 211}

$\begin{array}{ll}\text { plane group } & p 2 \\ \text { crystal class } & \text { oblique } \\ Z \begin{array}{l}Z \\ Z^{\prime}\end{array} & 1 \\ \begin{array}{l}\text { molecular } \\ \text { symmetry }\end{array} & 0.5 \\ \text { site symmetry } & 22 \\ \begin{array}{l}\text { molecular } \\ \text { weight }(D a)\end{array} & 13032.35 \\ \text { multicomponent } & \text { yes }\end{array}$

Lensen, M. C., van Dingenen, S. J. T., Elemans, J. A. A. W., Dijkstra, H. P., van Klink, G. P. M., van Koten, G., Gerritsen, J. W., Speller, S., nolte, R. J. M. \& Rowan, A. E. Chem. Commun. 2004 762-763.

\section{Record 212}

R-2-eicosyloxy-5-(1-

methylheptyloxy)terephthalic acid 


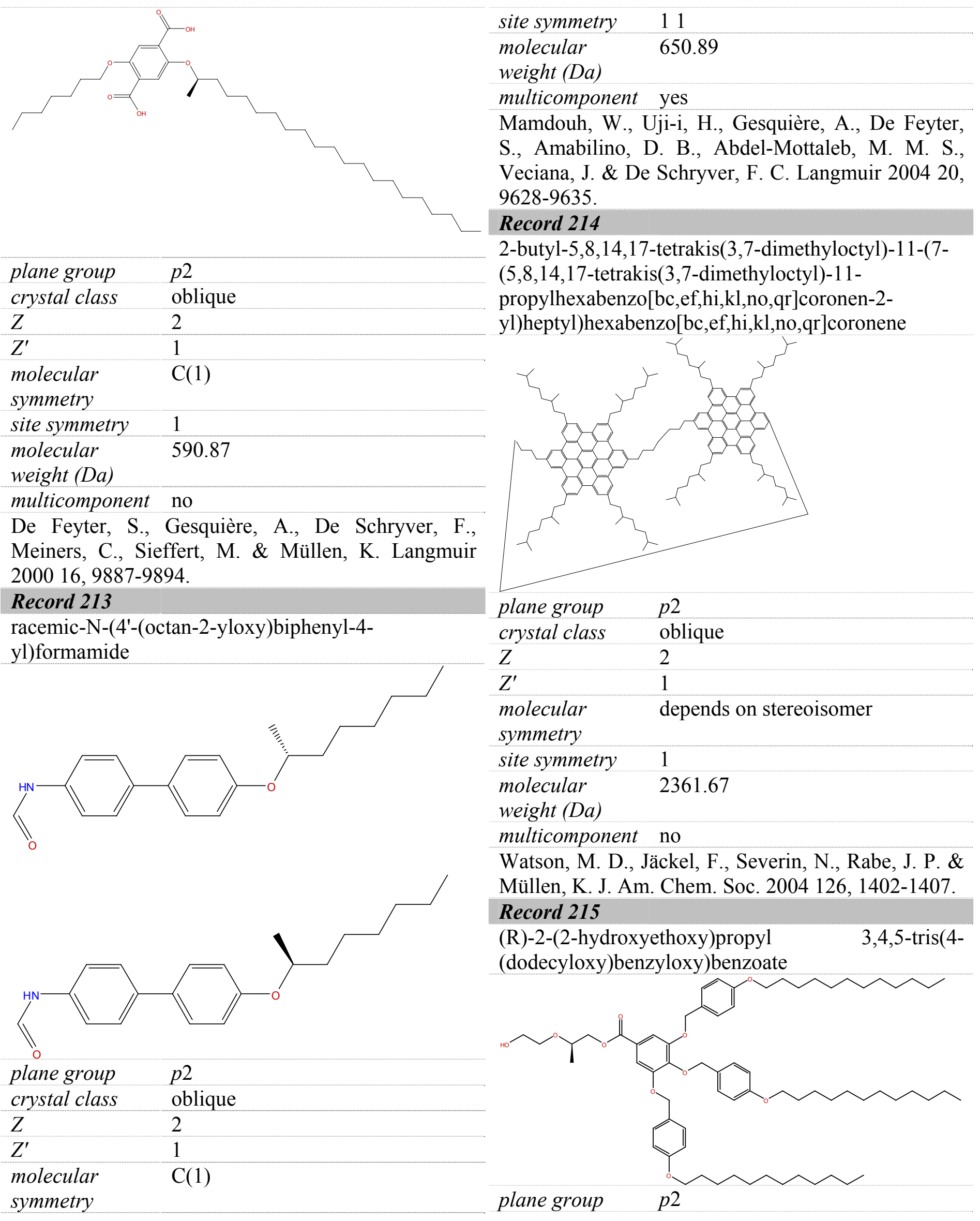

site symmetry $\quad 11$

molecular $\quad 650.89$

weight (Da)

multicomponent yes

Mamdouh, W., Uji-i, H., Gesquière, A., De Feyter, S., Amabilino, D. B., Abdel-Mottaleb, M. M. S., Veciana, J. \& De Schryver, F. C. Langmuir 200420 , 9628-9635.

\section{Record 214}

2-butyl-5,8,14,17-tetrakis(3,7-dimethyloctyl)-11-(7(5,8,14,17-tetrakis(3,7-dimethyloctyl)-11propylhexabenzo[bc,ef,hi,kl,no,qr]coronen-2(l) heptyl)hexabenzo[bc,ef,hi,kl,no,qr]coronene

plane group $\quad p 2$

crystal class oblique

Z 2

$Z^{\prime} \quad 1$

olecular

symmetry

site symmetry

molecular $\quad 2361.67$

weight (Da)

multicomponent no

Watson, M. D., Jäckel, F., Severin, N., Rabe, J. P. \& Müllen, K. J. Am. Chem. Soc. 2004 126, 1402-1407.

\section{Record 215}

(R)-2-(2-hydroxyethoxy)propyl

(dodecyloxy)benzyloxy)benzoate 


\begin{tabular}{ll} 
crystal class & oblique \\
\hline$Z$ & 2 \\
\hline$Z^{\prime}$ & 1 \\
\hline $\begin{array}{l}\text { molecular } \\
\text { symmetry }\end{array}$ & $\mathrm{C}(1)$ \\
site symmetry & 1 \\
\hline $\begin{array}{l}\text { molecular } \\
\text { weight (Da) }\end{array}$ & 1095.57 \\
multicomponent & no \\
Mamdouh, W., Uji-i, H., Dulcey, A. E., Percec, V., \\
De Feyter, S. \& De Schryver, F. C. Langmuir 2004 \\
20,7678-7685.
\end{tabular}

\section{Record 216}

(S)-2-(2-hydroxyethoxy)propyl 3,4,5-tris(4(dodecyloxy)benzyloxy)benzoate

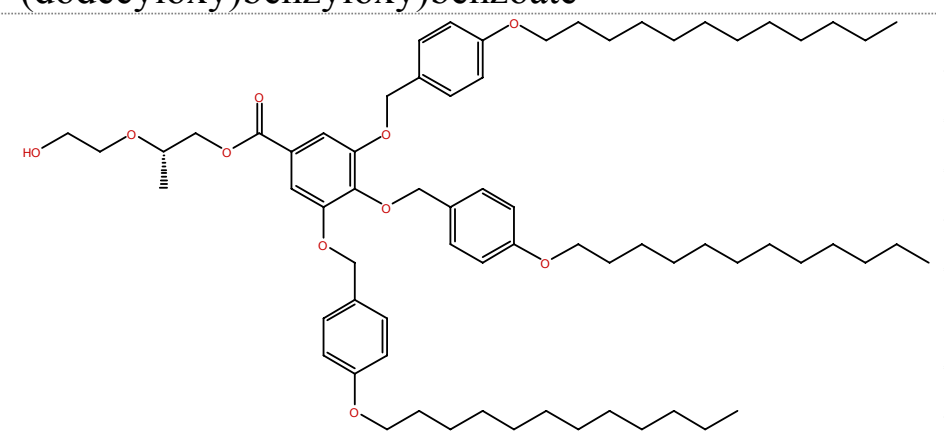

\begin{tabular}{ll} 
plane group & $p 2$ \\
crystal class & oblique \\
\hline$Z$ & 2 \\
\hline$Z^{\prime}$ & 1 \\
molecular & $\mathrm{C}(1)$ \\
symmetry & \\
site symmetry & 1 \\
molecular & 1095.57 \\
weight (Da) & \\
multicomponent & no \\
Mamdouh, W., Uji-i, H., Dulcey, A. E., Percec, V., \\
De Feyter, S. \& De Schryver, F. C. Langmuir 2004 \\
20,7678-7685.
\end{tabular}

\section{Record 217}

racemic-2-(2-hydroxyethoxy)propyl (dodecyloxy)benzyloxy)benzoate

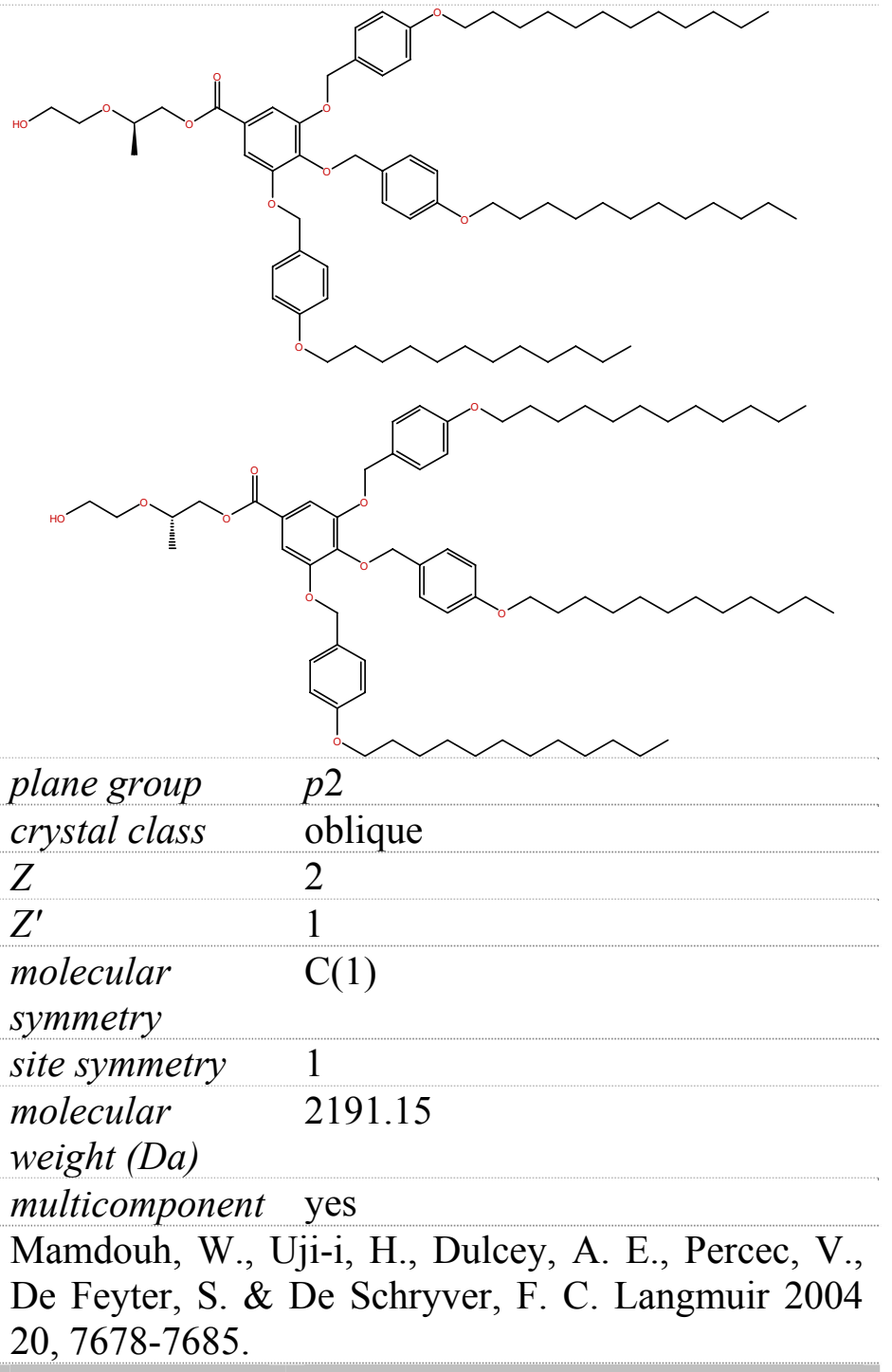

\section{Record 218}

2-(2-hydroxyethoxy)ethyl 3,4,5-tris(4(dodecyloxy)benzyloxy)benzoate and phenyloctane

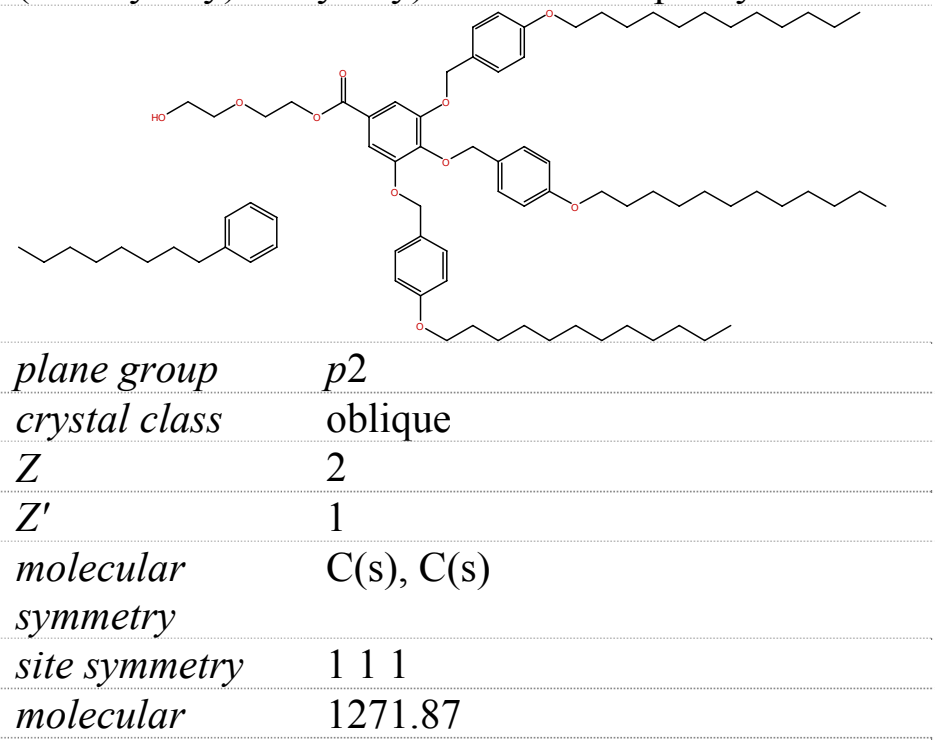


weight (Da)

multicomponent yes

Mamdouh, W., Uji-i, H., Dulcey, A. E., Percec, V., De Feyter, S. \& De Schryver, F. C. Langmuir 2004 20, 7678-7685.

\section{Record 219}

3,4,5-Tris[4-(dodecyl-1-oxy)benzyloxy]benzoic Acid (3)

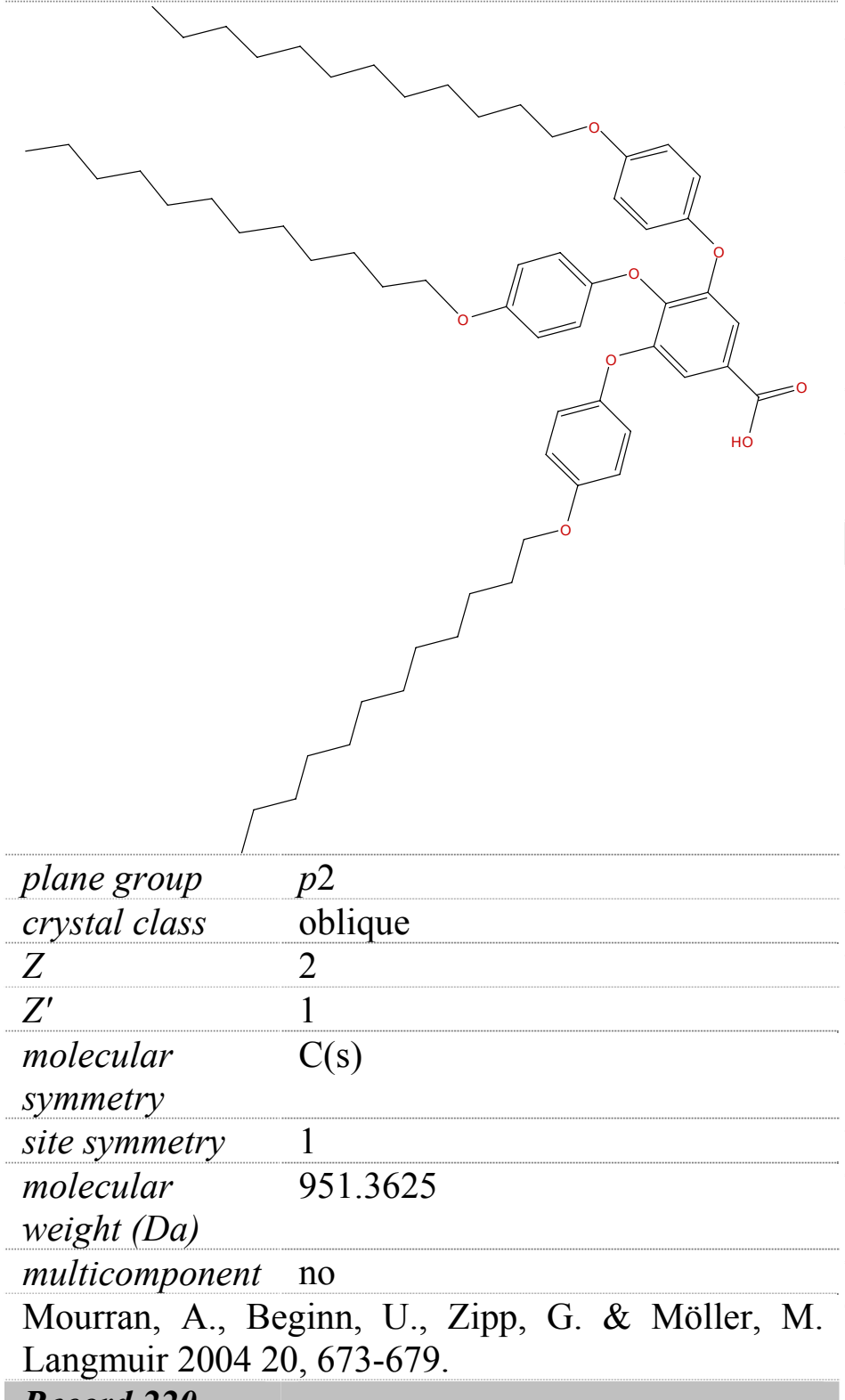

\section{Record 220}

1,3,8,10-tetramethyl-N,N'-dihexadecyl-quinacridone (TmQA-C16) and steric acid

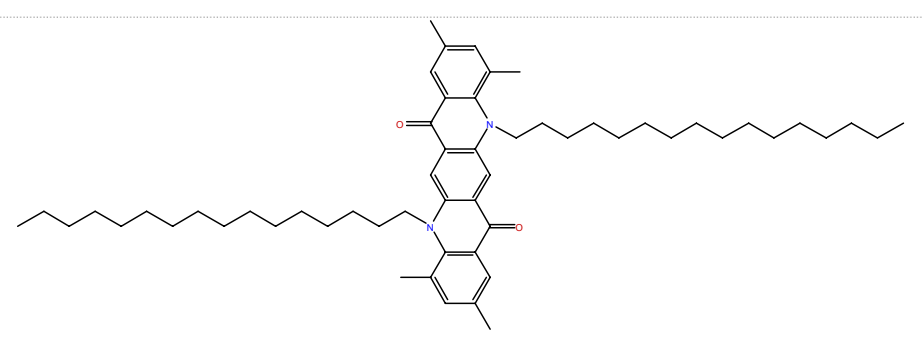

مlanegroup

plane group p2

crystal class oblique

$Z \quad 2$

$Z^{\prime} \quad 1$

molecular $\mathrm{C}(\mathrm{s}), \mathrm{C}(2 \mathrm{~h})$

symmetry

site symmetry 12

molecular $\quad 1101.76$

weight (Da)

multicomponent yes

Mu, Z., Wang, Z., Zhang, X., Ye, K. \& Wang, Y. J. Phys. Chem. B 2004 108, 19955-19959.

\section{Record 221}

(R)-4-(octan-2-yloxy)phenyl 4-formamidobenzoate

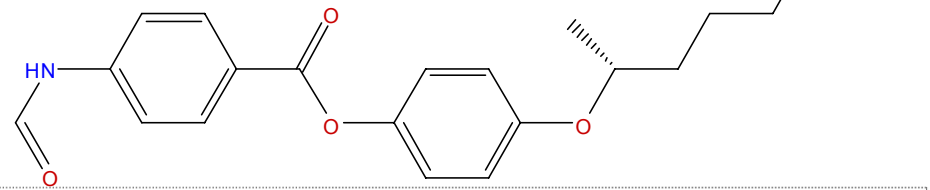

plane group p2

crystal class oblique

$Z \quad 2$

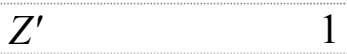

molecular $\quad \mathrm{C}(1)$

symmetry

site symmetry 1

molecular $\quad 369.45$

weight (Da)

multicomponent no

Mamdouh, W., Uji-i, H., Gesquière, A., De Feyter, S., Amabilino, D. B., Abdel-Mottaleb, M. M. S., Veciana, J. \& De Schryver, F. C. Langmuir 2004 20, 9628-9635.

\section{Record 222}

1,1'-(4,4'-(2,2'-bipyridine-5,5'-diyl)bis(butane-4,1diyl))bis(3-octadecylurea) 


\begin{tabular}{|c|c|}
\hline plane group & $p 2$ \\
\hline crystal class & oblique \\
\hline$Z$ & 1 \\
\hline & 0.5 \\
\hline $\begin{array}{l}\text { molecular } \\
\text { symmetry }\end{array}$ & $C(2 v)$ \\
\hline site symmetry & 2 \\
\hline $\begin{array}{l}\text { molecular } \\
\text { weight }(D a)\end{array}$ & 889.43 \\
\hline \multicolumn{2}{|c|}{ multicomponent no } \\
\hline \multicolumn{2}{|c|}{$\begin{array}{l}\text { De Feyter, S., Abdel-Mottaleb, M. M. S., } \\
\text { Schuurmans, N., Verkuijl, B. J. V., Van Esch, J. H., } \\
\text { Feringa, B. L. \& De Schryver, F. C. Chem.-Eur. J } \\
2004 \text { 10, 1124-1132. }\end{array}$} \\
\hline \multicolumn{2}{|l|}{ Record 223} \\
\hline \multicolumn{2}{|c|}{$\begin{array}{l}\text { 2,3,9,10-tetrakis(dodecyloxy)-5,12- } \\
\text { dimethylquinolino[2,3-b]acridine- } 7,14(5 \mathrm{H}, 12 \mathrm{H})- \\
\text { dione }\end{array}$} \\
\hline
\end{tabular}

plane group $\quad p 2$

crystal class oblique

$\begin{array}{ll}Z & 1 \\ Z^{\prime} & 0.5\end{array}$

molecular $\quad \mathrm{C}(2 \mathrm{~h})$

symmetry

site symmetry 2

molecular $\quad 1077.65$

weight (Da)

multicomponent no

De Feyter, S., Gesquière, A., De Schryver, F. C., Keller, U. \& Müllen, K. Chem. Mater. 2002 14, 989997.

\section{Record 224}

5,12-dimethyl-2,9-bis(2-

undecyltridecyloxy)quinolino[2,3-b]acridine-

7,14(5H,12H)-dione

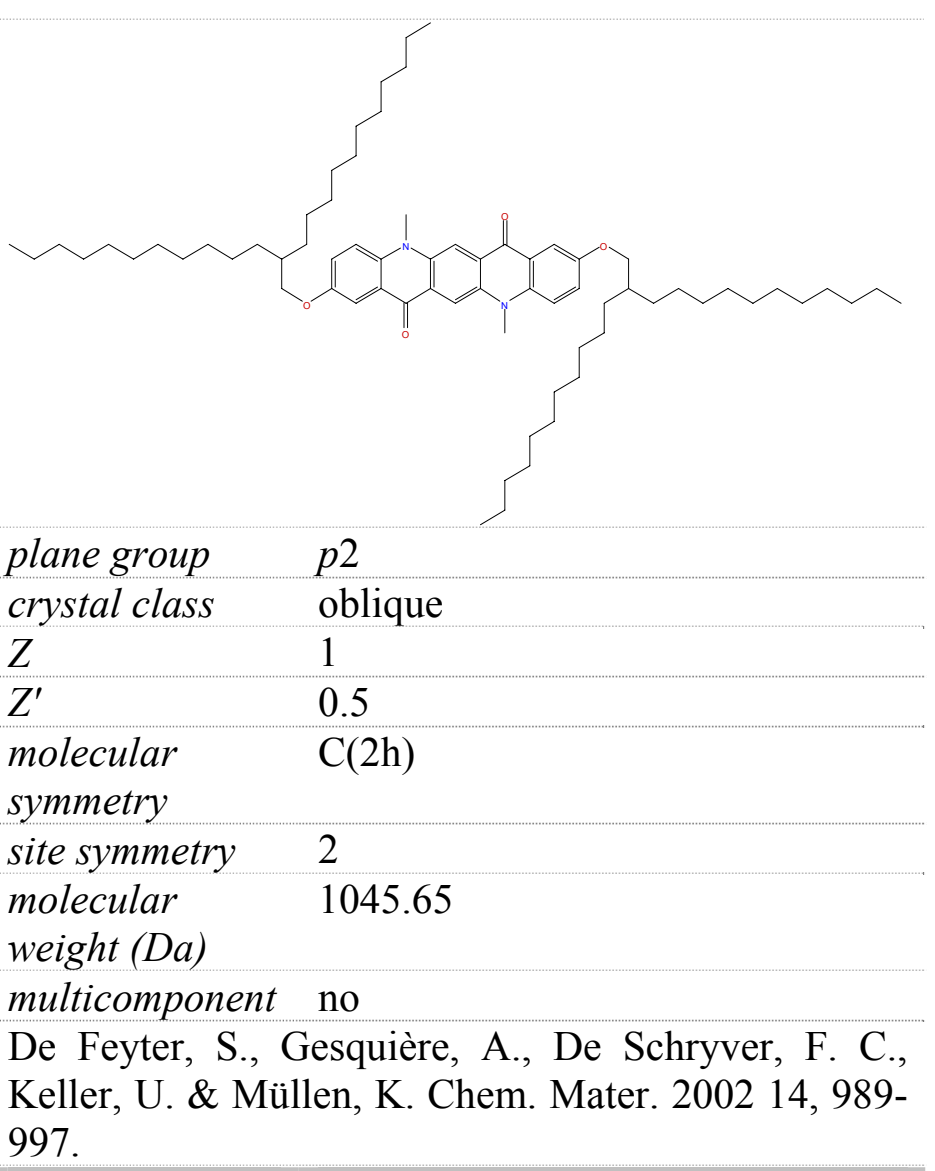

\section{Record 225}

bis(5-

dodecylsalicylidene)ethylenediaminato)nickel(II)

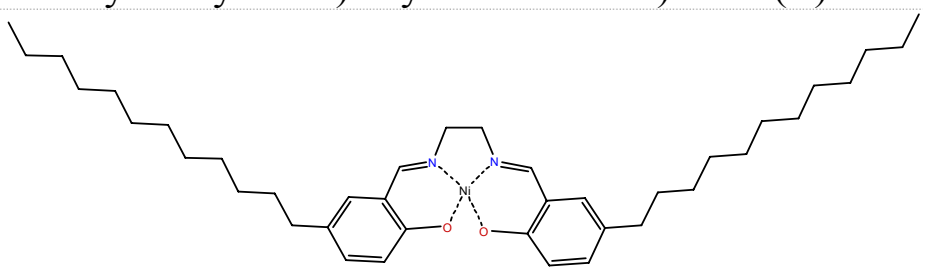

plane group $\quad p 2$

crystal class oblique

$Z \quad 2$

$Z^{\prime} \quad 1$

molecular $\quad \mathrm{C}(2 \mathrm{v})$

symmetry

site symmetry 1

molecular $\quad 661.63$

weight (Da)

multicomponent no

Sakata, I. \& Miyamura, K. Chem. Commun. 2003 156-157.

Record 226

hexakis(porphyrinato)benzene (H21) 


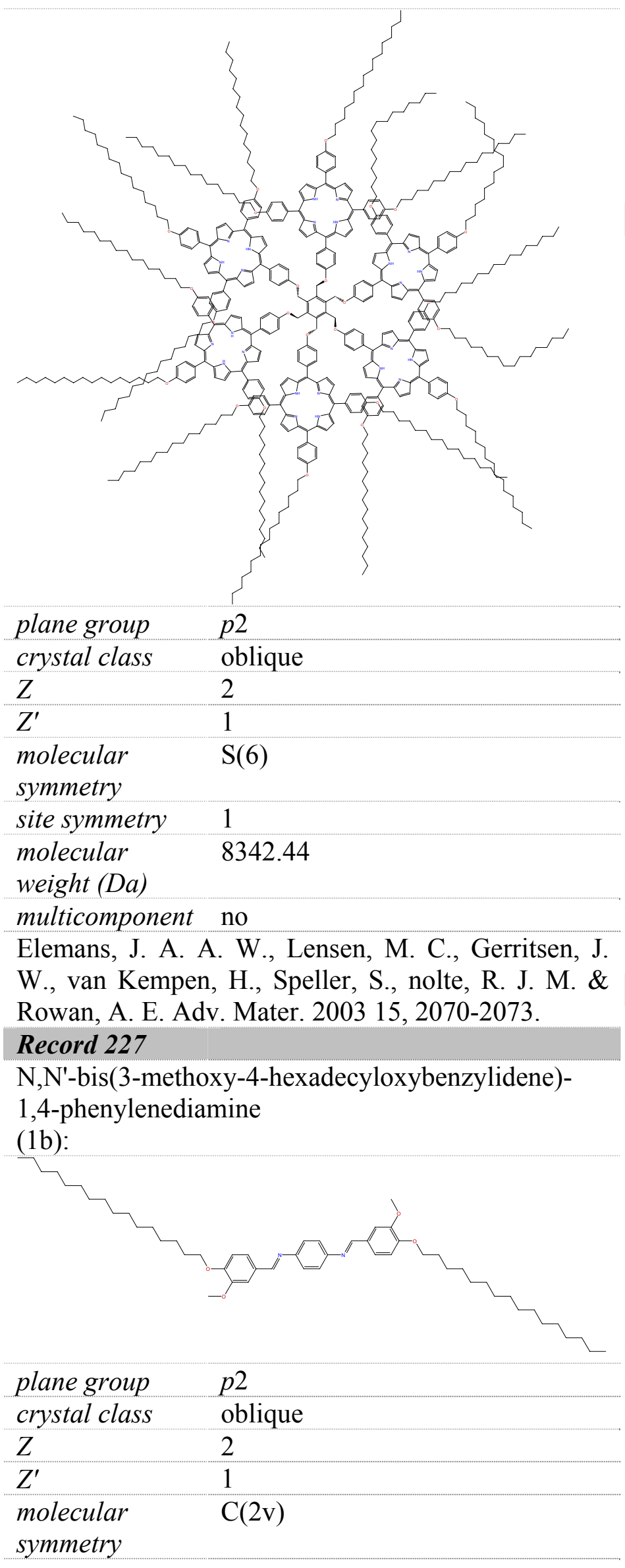

site symmetry $\quad 1$

molecular 825.26

weight (Da)

multicomponent no

Mu, Z.-C., Kong, J.-F., Wang, Y., Ye, L., Yang, G.D. \& Zhang, X. ChemPhysChem 2004 5, 202-208.

\section{Record 228}

N,N'-bis(3-methoxy-4-docosyloxybenzylidene)-1,4phenylenediamine (1c):

\begin{tabular}{ll} 
plane group & $p 2$ \\
crystal class & oblique \\
$Z$ & 1 \\
$Z^{\prime}$ & 0.5 \\
$\begin{array}{l}\text { molecular } \\
\text { symmetry }\end{array}$ & $\mathrm{C}(2 \mathrm{~h})$ \\
site symmetry & 2 \\
$\begin{array}{l}\text { molecular } \\
\text { weight }(D a)\end{array}$ & 993.57 \\
multicomponent & no \\
\hline
\end{tabular}

Mu, Z.-C., Kong, J.-F., Wang, Y., Ye, L., Yang, G.D. \& Zhang, X. ChemPhysChem 2004 5, 202-208.

\section{Record 229}

1-(4-amino-6-(4-(2,5-bis((S)-2-methylbutoxy)-4(3,4,5-tris(dodecyloxy)styryl)styryl)phenyl)-1,3,5triazin-2-yl)-3-butylurea

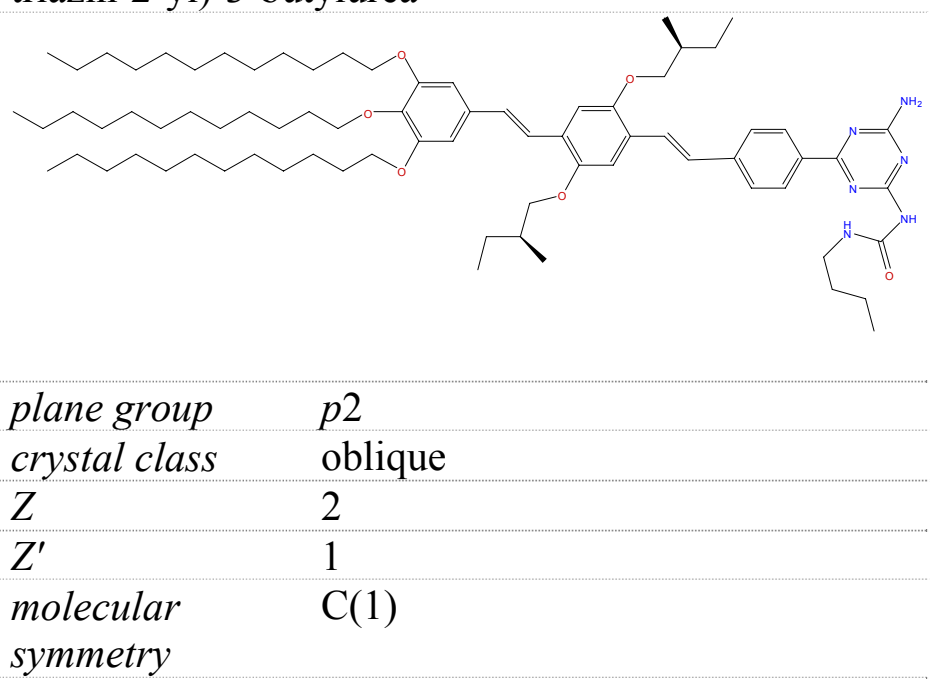


site symmetry 1

molecular

1215.82

weight (Da)

multicomponent no

Gesquière, A., Jonkheijm, P., Hoeben, F. J. M., Schenning, A. P. H. J., De Feyter, S., De Schryver, F. C. \& Meijer, E. W. Nano Lett. 2004 4, 1175-1179.

\section{Record 230}

N,N'-bis(4-hexadecyloxybenzylidene)-1,4phenylenediamine $(2 \mathrm{~b})$ :

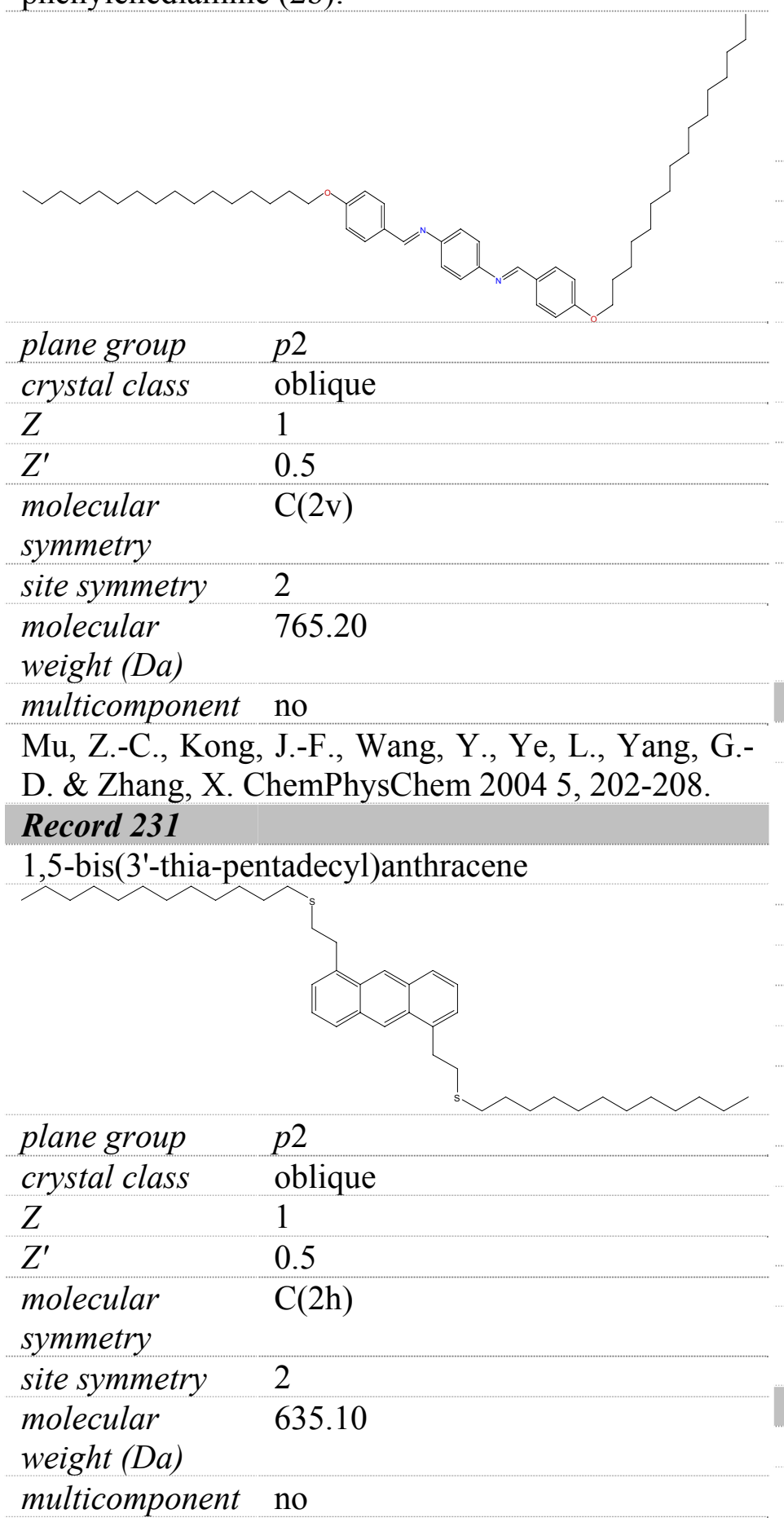

Wei, Y., Kannappan, K., Flynn, G. W. \& Zimmt, M. B. J. Am. Chem. Soc. 2004 126, 5318-5322.

\section{Record 232}

racemic (E)-2-nitro-4-(octan-2-yloxy)phenyl 4'-(dec4-enyloxy)biphenyl-4-carboxylate

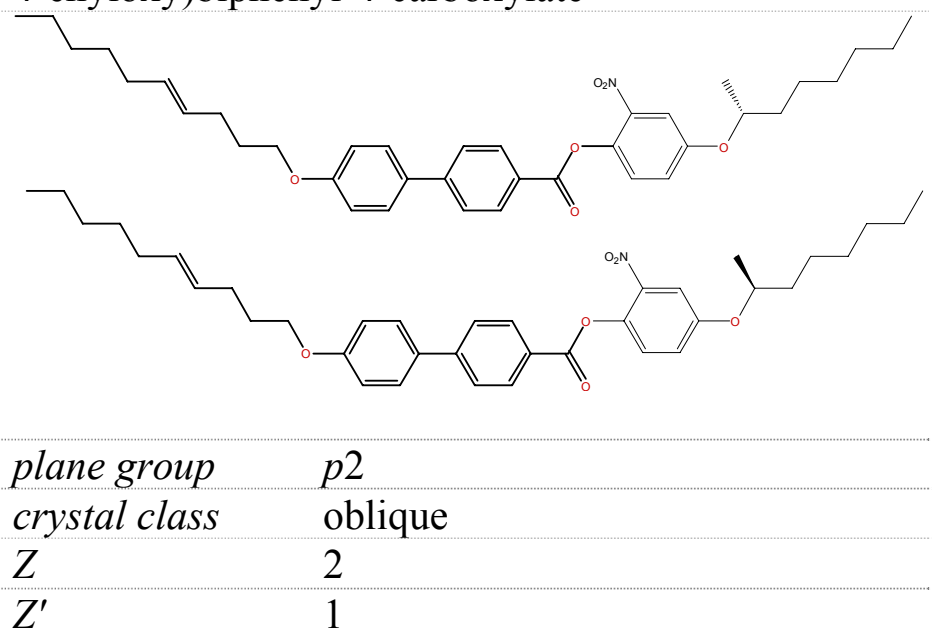

molecular $\quad \mathrm{C}(1)$

symmetry

site symmetry 1

molecular $\quad 1203.54$

weight (Da)

multicomponent yes

Walba, D. M., Stevens, F. \& Dyer, D. J. Polym.

Prepr. (Am. Chem. Soc., Div. Polym. Chem.) 1996 37, 489-490.

\section{Record 233}

eladic acid

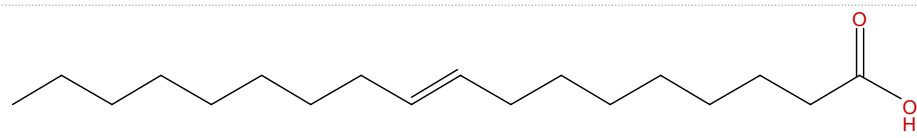

plane group p2

crystal class oblique

$Z \quad 2$

$Z^{\prime} \quad 1$

molecular $\quad \mathrm{C}(\mathrm{s})$

symmetry

site symmetry 1

molecular $\quad 282.46$

weight (Da)

multicomponent no

Hibino, M., Sumi, A. \& Hatta, I. Jpn. J. Appl. Phys., Part 11995 34, 3354-3359.

\section{Record 234}

5-nonadecyl-2-(5-nonadecylpyridin-2-yl)pyridine 


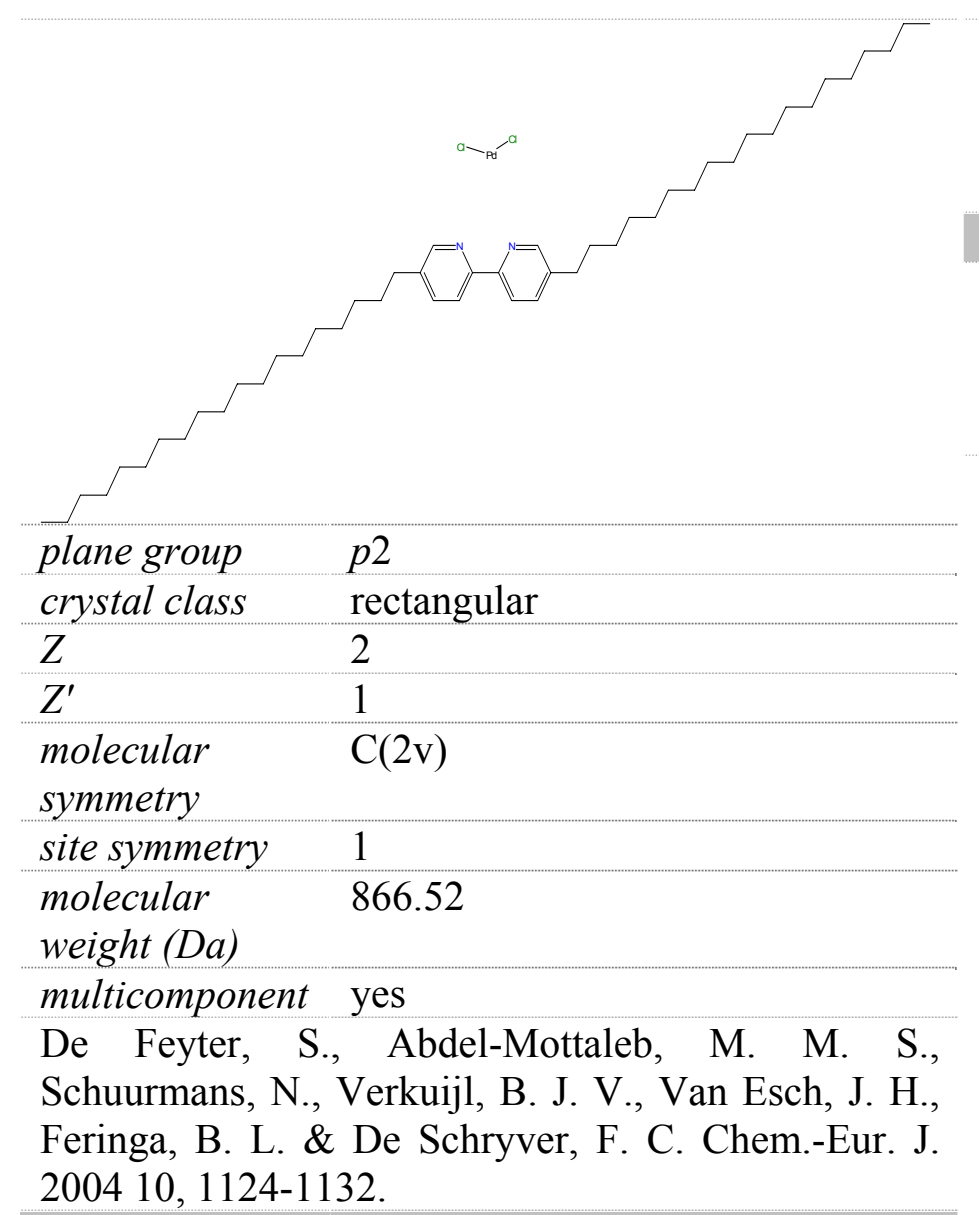

\section{Record 235}

5-methyl-2-(5-nonadecylpyridin-2-yl)pyridine

oblique
crystal class
$Z$

De Feyter, S.; Abdel-Mottaleb, M. M. S.; Schuurmans, N.; Verkuijl, B. J. V.; Van Esch, J. H.; Feringa, B. L.; De Schryver, F. C. Chem.-Eur. J. 2004, 10, 1124-1132.

\section{Record 236}

hexakis $((9,10$-dimethoxy-9,10-dihydroanthracen-2yl)methyl) 4,4 ',4",4"',4"', 4 "'"'(hexabenzo[bc,ef,hi,kl,no,qr]coronene2,5,8,11,14,17-hexayl)hexabutanoate

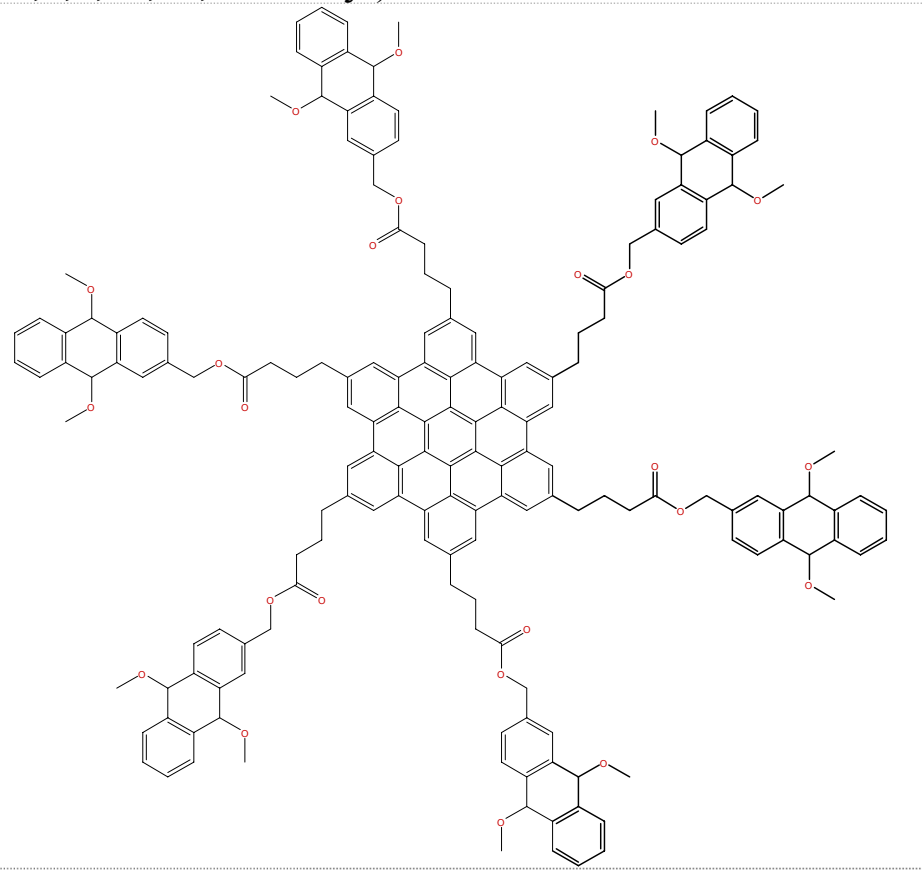

plane group p2

crystal class oblique

$\begin{array}{ll}Z & 1 \\ Z^{\prime} & 0.5 \\ \text { molecular } & \mathrm{C}(6 \mathrm{~h}) \\ \text { symmetry } & \\ \text { site symmetry } & 2 \\ \text { molecular } & 2552.97\end{array}$

weight (Da)

multicomponent no

Samorì, P., Yin, X., Tchebotareva, N., Wang, Z., Pakula, T., Jäckel, F., Watson, M. D., Venturini, A., Müllen, K. \& Rabe, J. P. J. Am. Chem. Soc. 2004 126, 3567-3575.

\section{Record 237}

(E)-2-(but-3-enyl)-5,8,14,17-tetrakis(3,7-

dimethyloctyl)-11-(7-(5,8,14,17-tetrakis(3,7dimethyloctyl)-11propylhexabenzo[bc,ef,hi,kl,no,qr]coronen-2-yl)hept3-enyl)hexabenzo[bc,ef,hi,kl,no,qr]coronene 


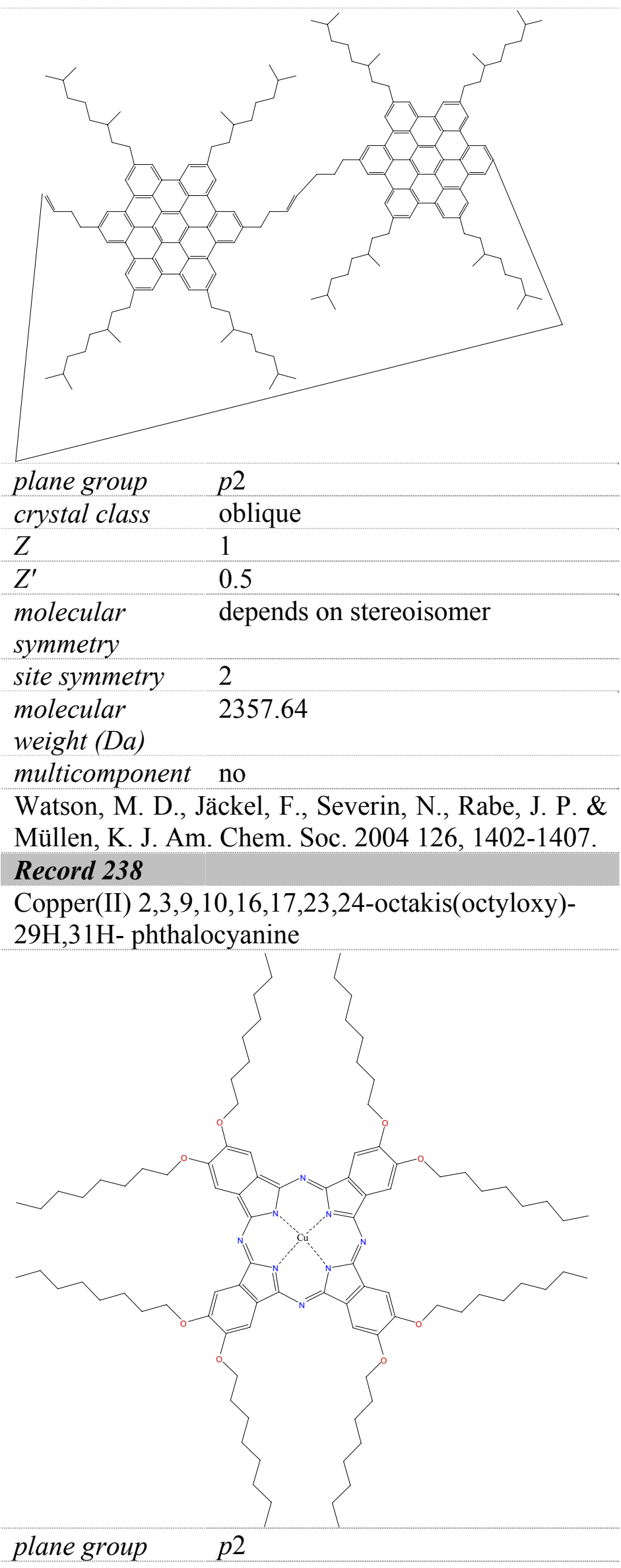

\begin{tabular}{|c|c|}
\hline crystal class & oblique \\
\hline$Z$ & 1 \\
\hline$Z^{\prime}$ & 0.5 \\
\hline $\begin{array}{l}\text { molecular } \\
\text { symmetry }\end{array}$ & $\mathrm{D}(4 \mathrm{~h})$ \\
\hline site symmetry & 2 \\
\hline $\begin{array}{l}\text { molecular } \\
\text { weight }(D a)\end{array}$ & 1601.77 \\
\hline multicomponen & no \\
\hline
\end{tabular}

\section{Record 239}

N,N'-bis(4-hexyloxybenzylidene)-1,4phenylenediamine $(2 \mathrm{a})$ :

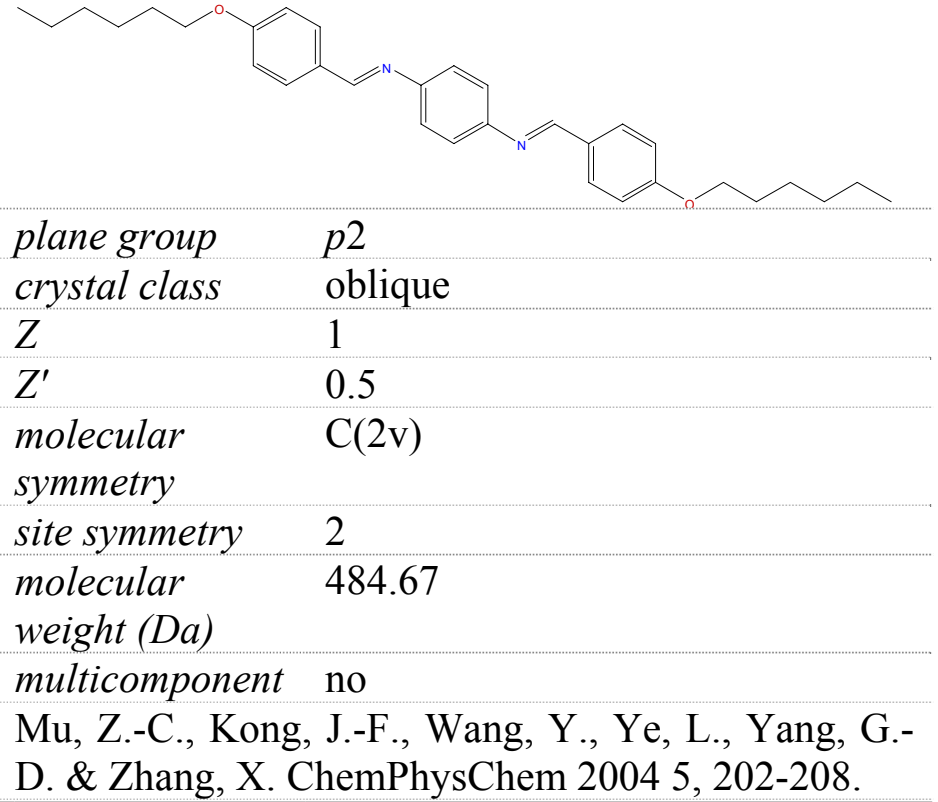

\section{Record 240}

brassidic acid

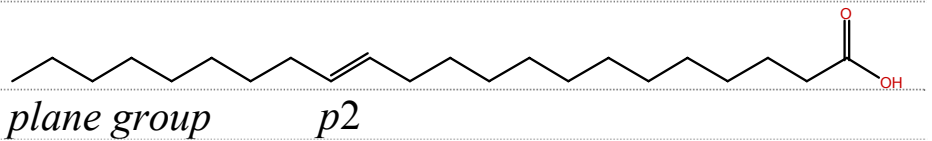

crystal class oblique

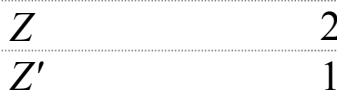

molecular $\quad \mathrm{C}(\mathrm{s})$

symmetry

site symmetry 1

molecular $\quad 338.57$

weight (Da)

multicomponent no

Canet, D., Guillain, F. \& Sanson, A. J. Trace Microprobe Tech. 1995 13, 361-362.

\section{Record 241}


1,2-bis(4-(dodecyloxy)phenyl)diazene

C12(AZO)C12

\begin{tabular}{ll} 
plane group & $p 2$ \\
\hline crystal class & oblique \\
\hline$Z$ & 1 \\
\hline$Z^{\prime}$ & 0.5 \\
\hline $\begin{array}{l}\text { molecular } \\
\text { symmetry }\end{array}$ & $\mathrm{C}(2 \mathrm{~h})$ \\
\hline site symmetry & 2 \\
\hline $\begin{array}{l}\text { molecular } \\
\text { weight }(\mathrm{Da})\end{array}$ & 550.86 \\
multicomponent & no \\
\hline
\end{tabular}

Grim, P. C. M., Vanoppen, P., Rücker, M., De Feyter, S., Valiyaveettil, S., Moessner, G., Müllen, K. \& De Schryver, F. C. J. Vac. Sci. Technol., B 1997 15, 1419-1424.

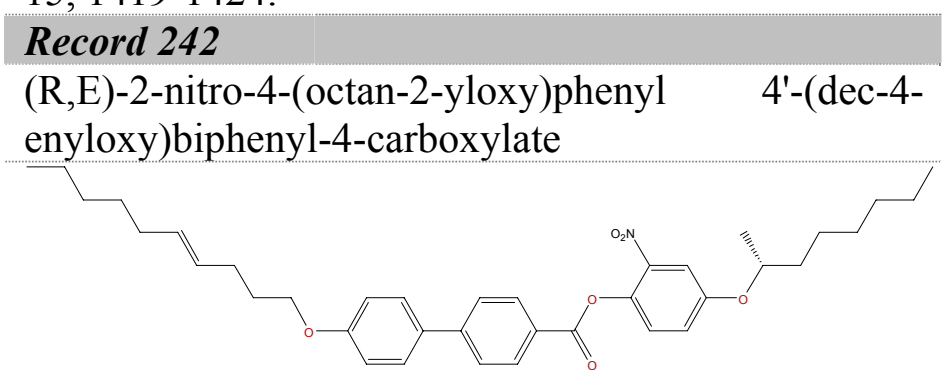

\begin{tabular}{ll}
\hline plane group & $p 2$ \\
\hline crystal class & oblique \\
\hline$Z$ & 2 \\
\hline$Z^{\prime}$ & 1 \\
\hline $\begin{array}{l}\text { molecular } \\
\text { symmetry }\end{array}$ & $\mathrm{C}(1)$ \\
\hline site symmetry & 1 \\
\hline $\begin{array}{l}\text { molecular } \\
\text { weight }(\mathrm{Da})\end{array}$ & 601.77 \\
\hline multicomponent & no \\
\hline
\end{tabular}

Stevens, F., Dyer, D. J. \& Walba, D. M. Angew. Chem., Int. Edit. 1996 35, 900-901.

\section{Record 243}

(S,E)-2-nitro-4-(octan-2-yloxy)phenyl 4'-(dec-4enyloxy)biphenyl-4-carboxylate

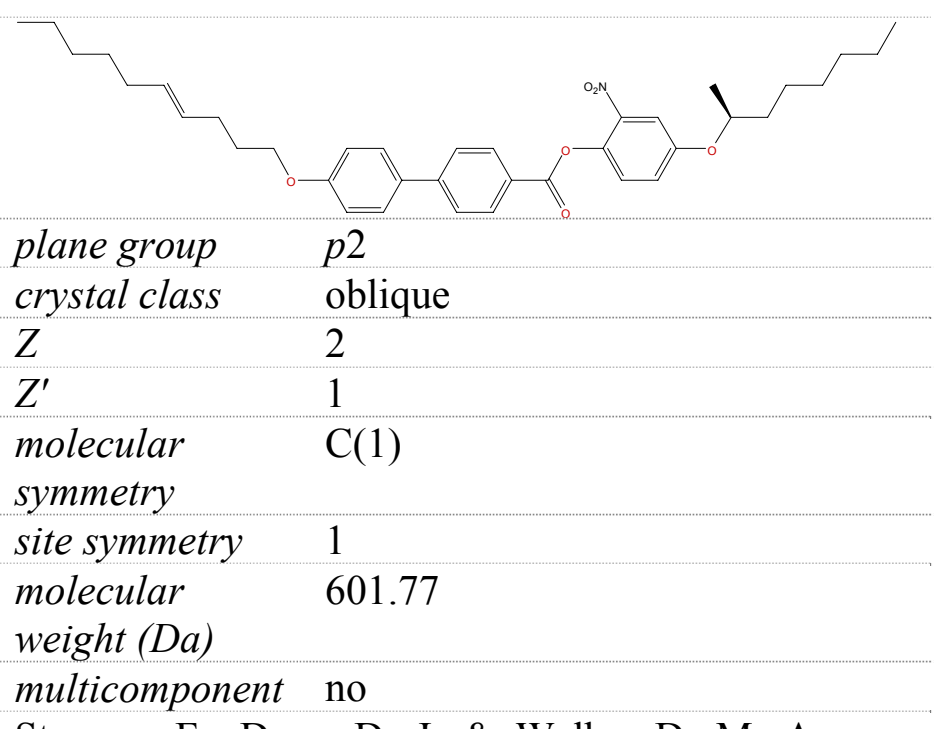

Stevens, F., Dyer, D. J. \& Walba, D. M. Angew. Chem., Int. Edit. 1996 35, 900-901.

\section{Record 244}

diacetylene isophthalic acid derivative 5-(tricosa10,12-diynyloxy)benzene-1,3-dioic acid

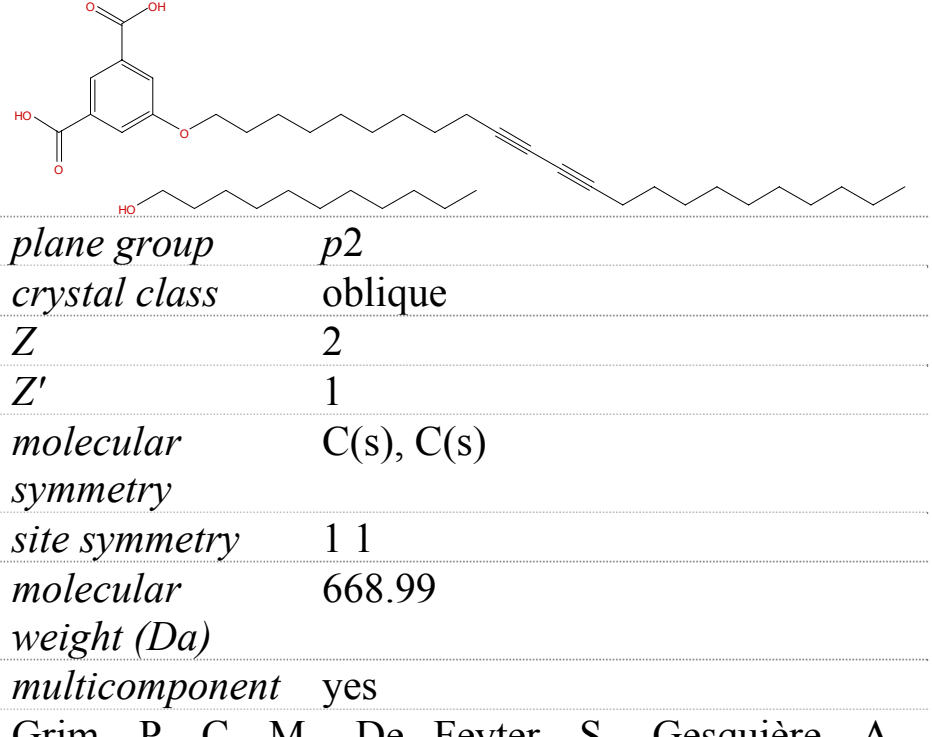

Grim, P. C. M., De Feyter, S., Gesquière, A., Vanoppen, P., Rücker, M., Valiyaveettil, S., Moessner, G., Müllen, K. \& De Schryver, F. C. Angew. Chem., Int. Edit. 1997 36, 2601-2603.

\section{Record 245}

(S)-2-nitro-4-(octan-2-yloxy)phenyl

4 '(decyloxy)biphenyl-4-carboxylate with (S,E)-2-nitro4-(octan-2-yloxy)phenyl 4'-(dec-4-enyloxy)biphenyl4-carboxylate 


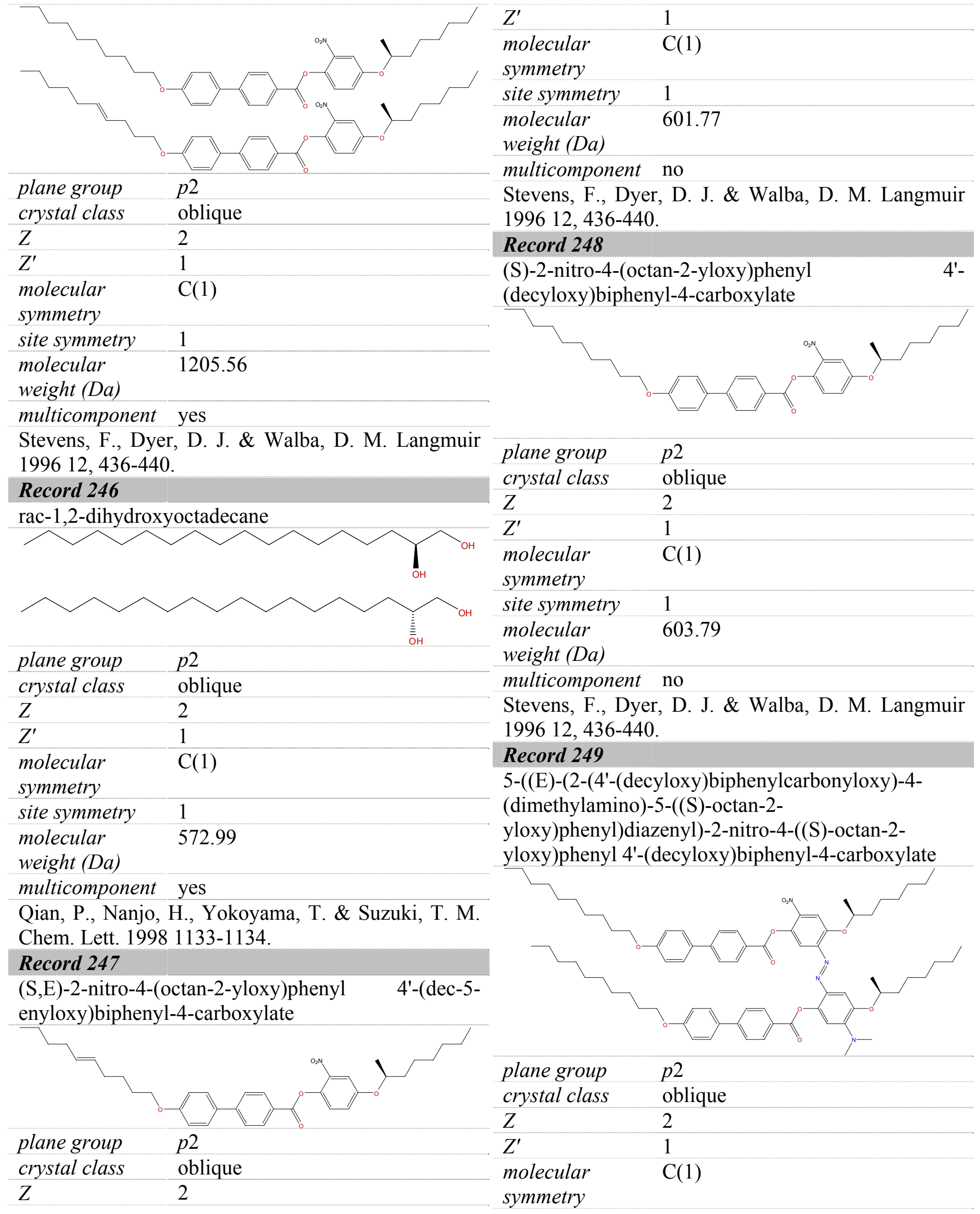


site symmetry $\quad 1$

molecular

1231.64

weight $(\mathrm{Da})$

multicomponent no

Stevens, F., Dyer, D. J., Müller, U. \& Walba, D. M. Langmuir 1996 12, 5625-5629.

\section{Record 250}

5-((2-(4'-(decyloxy)biphenylcarbonyloxy)-4-

(dimethylamino)-5-((S)-octan-2-

yloxy)phenyl)ethynyl)-2-nitro-4-((S)-octan-2-

yloxy)phenyl 4'-(decyloxy)biphenyl-4-carboxylate

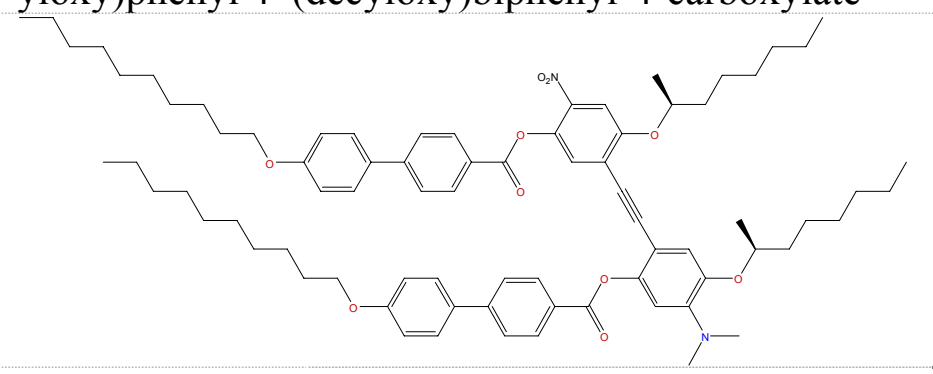

plane group p2

crystal class oblique

$Z^{2} \quad 2$

$Z^{\prime} \quad 1$

molecular $\quad \mathrm{C}(1)$

symmetry

site symmetry 1

molecular $\quad 1227.65$

weight (Da)

multicomponent no

Stevens, F., Dyer, D. J., Müller, U. \& Walba, D. M.

Langmuir 1996 12, 5625-5629.

\section{Record 251}

(E)-5-(12-(4-)( $4-$

(dodecyloxy)phenyl)diazenyl)phenoxy)dodecyloxy)is ophthalic acid (trans-C12(AZO)C12ISA) with undecanol

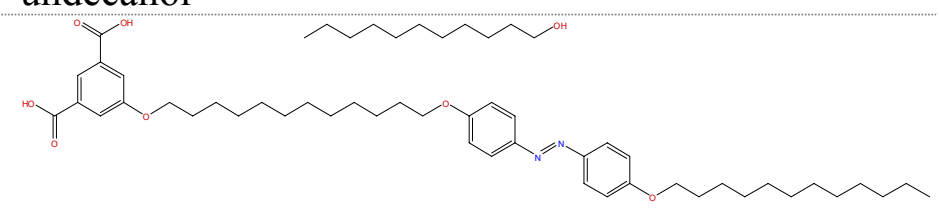

plane group $\quad p 2$

crystal class oblique

$\begin{array}{ll}Z & 2 \\ Z^{\prime} & 1\end{array}$

molecular $\mathrm{C}(\mathrm{s}), \mathrm{C}(\mathrm{s})$

symmetry

site symmetry 11 molecular

903.28

weight (Da)

multicomponent yes

Vanoppen, P., Grim, P. C. M., Rücker, M., De Feyter, S., Moessner, G., Valiyaveettil, S., Müllen, K. \& De Schryver, F. C. J. Phys. Chem. 1996 100, 19636-19641.

\section{Record 252}

(Z)-5-(12-(4-((4-

(dodecyloxy)phenyl)diazenyl)phenoxy)dodecyloxy)is ophthalic acid (cis-C12(AZO)C12ISA)

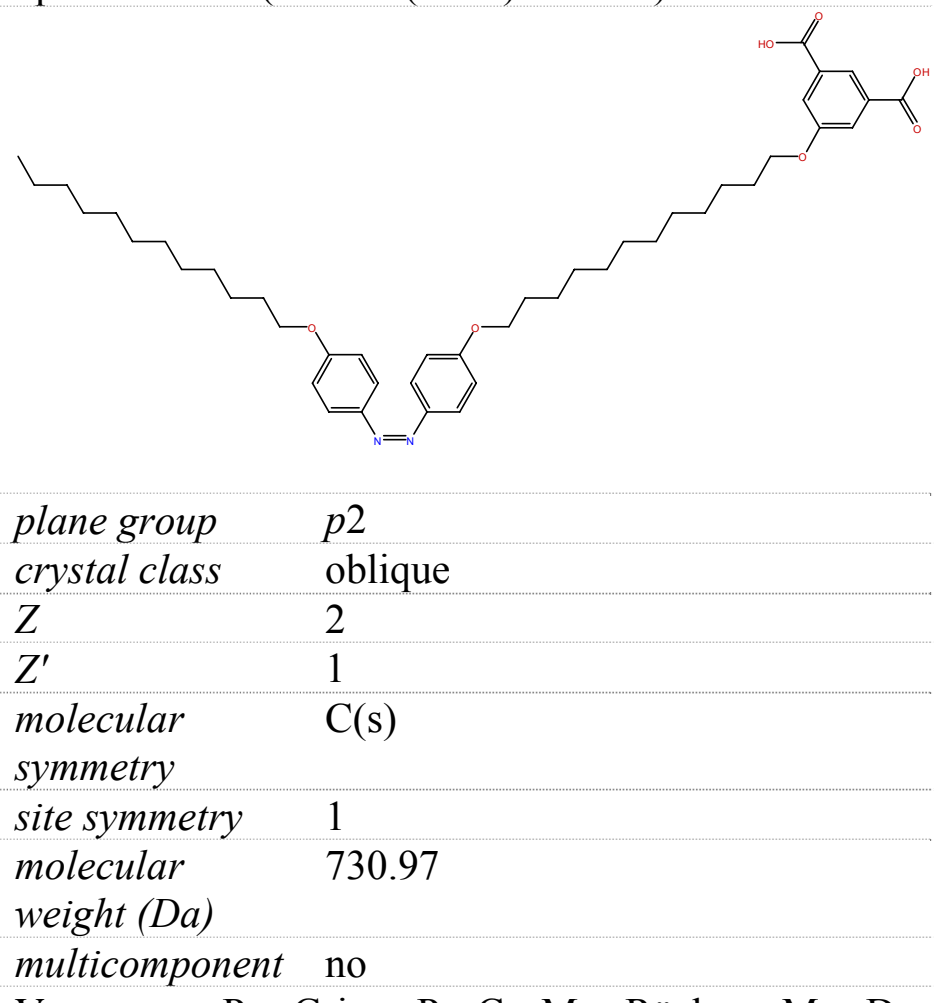

Vanoppen, P., Grim, P. C. M., Rücker, M., De Feyter, S., Moessner, G., Valiyaveettil, S., Müllen, K. \& De Schryver, F. C. . Phys. Chem. 1996 100, 19636-19641.

\section{Record 253}

(E)-5,5'-(12,12'-(4,4'-(diazene-1,2-diyl)bis(4,1phenylene))bis(oxy)bis(dodecane-12,1diyl))diisophthalic acid (ISA C12(ZAO)C12 ISA)

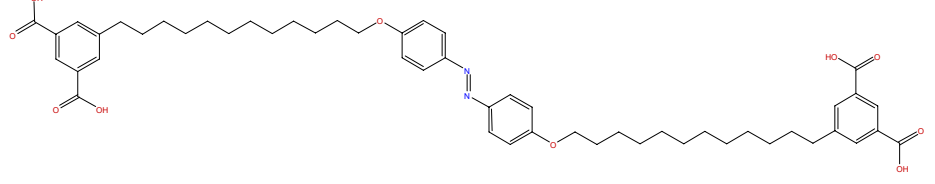

plane group $\quad p 2$

crystal class oblique 


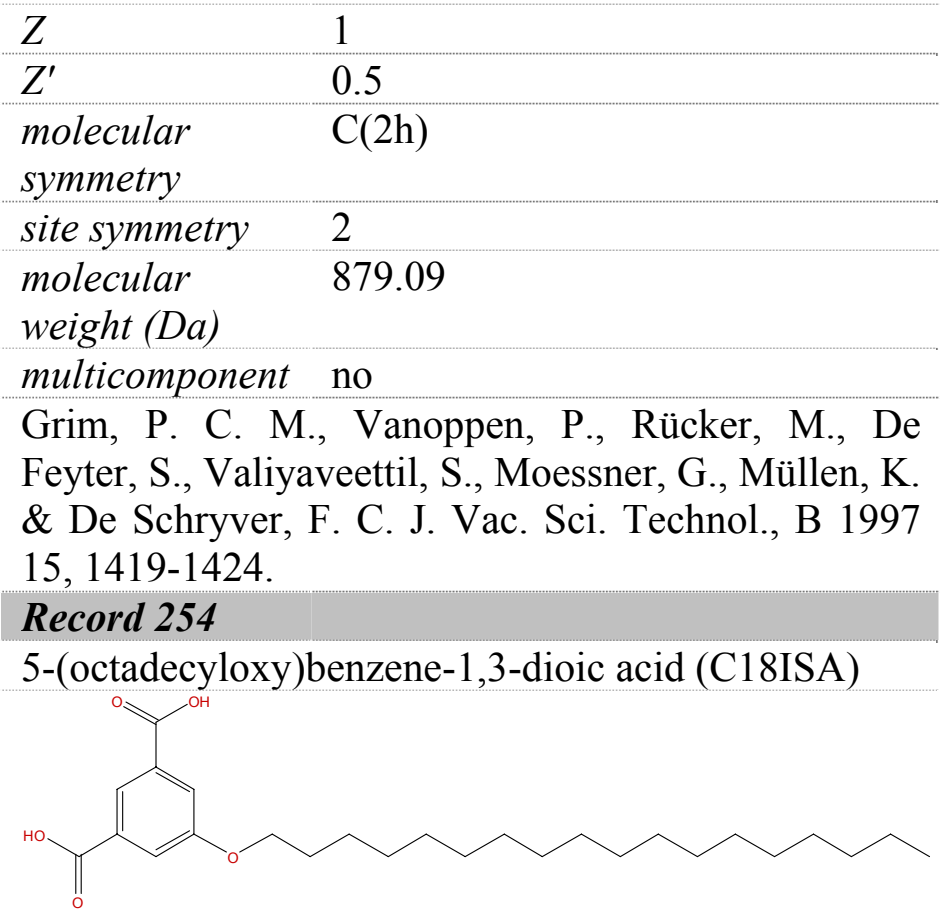

plane group $\quad p 2$

crystal class oblique

$\begin{array}{ll}Z & 2 \\ Z^{\prime} & 1\end{array}$

molecular $\quad \mathrm{C}(\mathrm{s})$

symmetry

site symmetry 1

molecular $\quad 434.61$

weight (Da)

multicomponent no

Vanoppen, P., Grim, P. C. M., Rücker, M., De Feyter, S., Moessner, G., Valiyaveettil, S., Müllen, K. \& De Schryver, F. C. J. Phys. Chem. 1996 100, 19636-19641.

\section{Record 255}

(S)-2-nitro-4-(octan-2-yloxy)phenyl

4'-

(decyloxy)biphenyl-4-carboxylate with (S,E)-2-nitro4-(octan-2-yloxy)phenyl 4'-(dec-4-enyloxy)biphenyl4-carboxylate

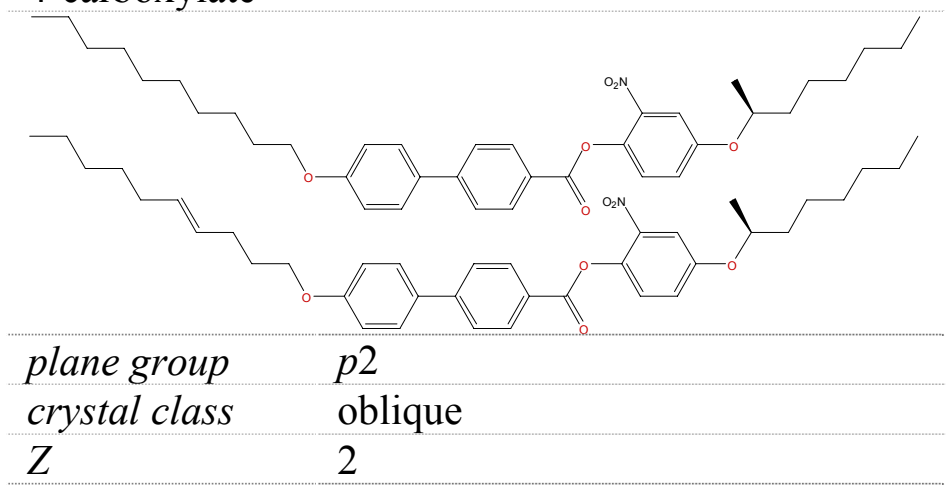

$Z^{\prime} \quad 1$

molecular $\quad \mathrm{C}(1)$

symmetry

site symmetry 1

molecular $\quad 1205.56$

weight $(D a)$

multicomponent yes

Stevens, F., Dyer, D. J. \& Walba, D. M. Langmuir 1996 12, 436-440.

\section{Record 256}

triacontanoic acid or melissic acid

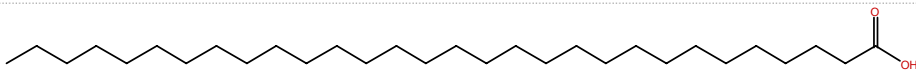

plane group $\quad p 2$

crystal class oblique

Z 2

$Z^{\prime} \quad 1$

molecular $\quad \mathrm{C}(\mathrm{s})$

symmetry

site symmetry 1

molecular $\quad 452.80$

weight $(D a)$

multicomponent no

Canet, D., Guillain, F. \& Sanson, A. J. Trace Microprobe Tech. 1995 13, 361-362.

\section{Record 257}

3-dodecyl-5-(4-dodecyl-5-(5-(5-(3-dodecyl-5-(4dodecyl-5-(5-(5-(3-dodecyl-5-(4-dodecyl-5-(5-(5-(3dodecylthiophen-2-yl)thiophen-2-yl)thiophen-2yl)thiophen-2-yl)thiophen-2-yl)thiophen-2-

yl)thiophen-2-

\begin{tabular}{ll} 
plane group & $p 2$ \\
\hline crystal class & oblique \\
\hline$Z$ & 1 \\
\hline$Z^{\prime}$ & 0.5 \\
\hline $\begin{array}{l}\text { molecular } \\
\text { symmetry }\end{array}$ & $\mathrm{C}(2 \mathrm{~h})$ \\
\hline site symmetry & 2 \\
\hline molecular & 2662.55 \\
\hline
\end{tabular}


weight (Da)

multicomponent no

Bäuerle, P., Fischer, T., Bidlingmeier, B., Stabel, A. \& Rabe, J. P. Angew. Chem., Int. Edit. 1995 34, 303307.

\section{Record 258}

hexadodecyldodecithiophene

3-dodecyl-5-(4-dodecyl-5-(5-(5-(3-dodecyl-5-(4dodecyl-5-(5-(5-(3-dodecylthiophen-2-yl)thiophen-2yl)thiophen-2-yl)thiophen-2-yl)thiophen-2-

yl)thiophen-2-yl)thiophen-2-yl)t

\begin{tabular}{ll} 
plane group & $p 2$ \\
crystal class & oblique \\
\hline$Z$ & 1 \\
\hline$Z^{\prime}$ & 0.5 \\
molecular & $\mathrm{C}(2 \mathrm{~h})$ \\
\hline
\end{tabular}

symmetry

site symmetry 2

molecular

1997.41

weight (Da)

multicomponent no

Bäuerle, P., Fischer, T., Bidlingmeier, B., Stabel, A. \& Rabe, J. P. Angew. Chem., Int. Edit. 1995 34, 303307.

\section{Record 259}

tetradodecyloctathiophene 3-dodecyl-5-(4-dodecyl-5(5-(5-(3-dodecylthiophen-2-yl)thiophen-2yl)thiophen-2-yl)thiophen-2-yl)-2-(5-(5-(3dodecylthiophen-2-yl)thiophen-2-yl)thiophen-2yl)thiophene

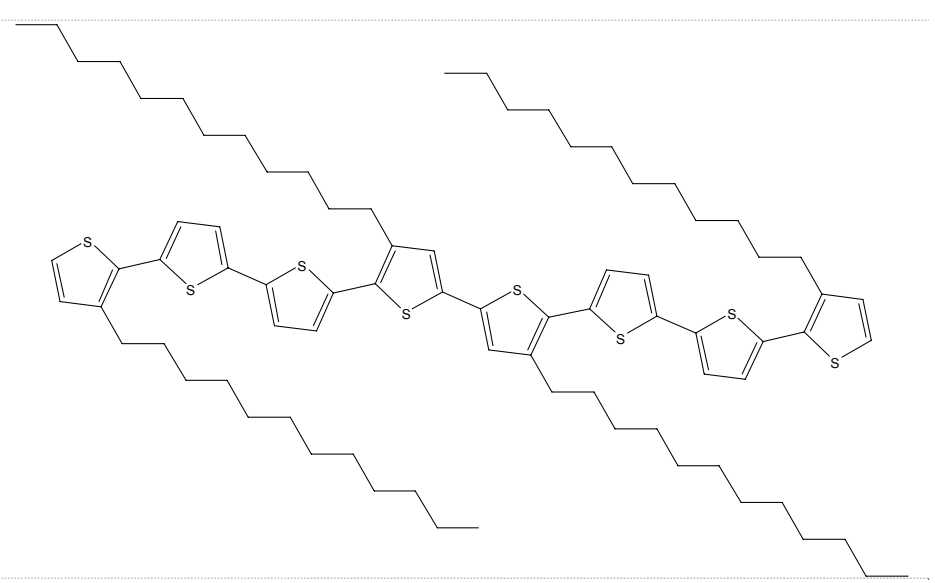

plane group $\quad p 2$

crystal class oblique

$Z \quad 1$

$Z^{\prime} \quad 0.5$

molecular $\mathrm{C}(2 \mathrm{~h})$

symmetry

site symmetry 2

molecular $\quad 1332.28$

weight $(\mathrm{Da})$

multicomponent no

Bäuerle, P., Fischer, T., Bidlingmeier, B., Stabel, A. \& Rabe, J. P. Angew. Chem., Int. Edit. 1995 34, 303307.

\section{Record 260}

3,3"'-Didodecyl-2,2':5',2":5",2'"-quaterthiophene 3,3"didodecylquaterthiophene (DD4T)

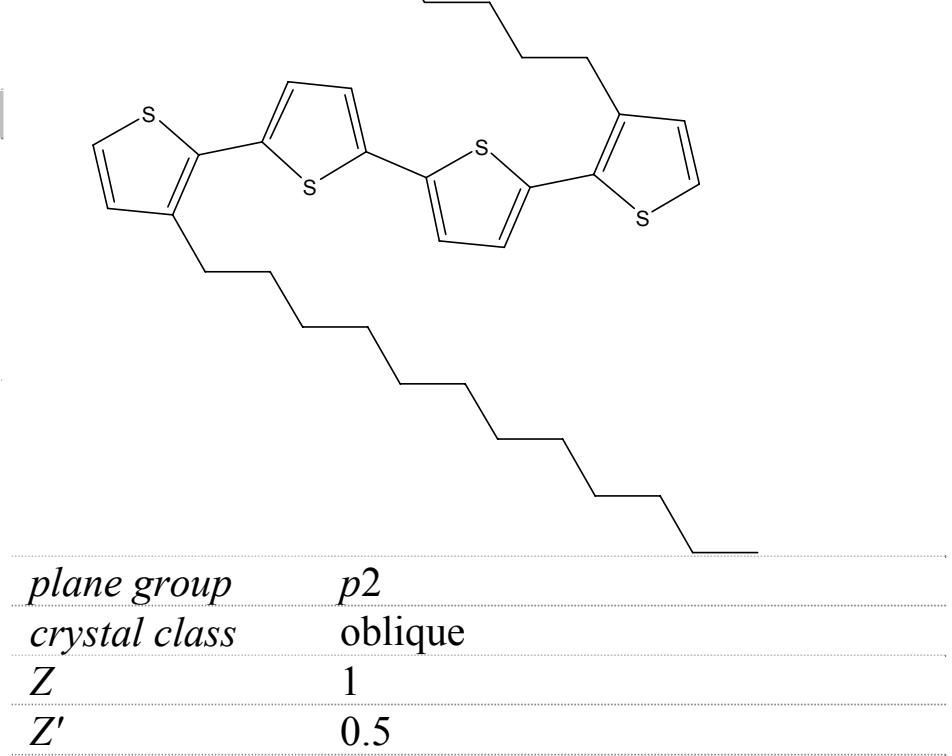


molecular

symmetry

site symmetry 2

molecular $\quad 667.15$

weight (Da)

multicomponent no

Bäuerle, P., Fischer, T., Bidlingmeier, B., Stabel, A. \& Rabe, J. P. Angew. Chem., Int. Edit. 1995 34, 303307.

\section{Record 261}

2-pyridone-5-carboxylic-acid-[4'-(noctyloxy)phenyl]-ester (PCA-E)

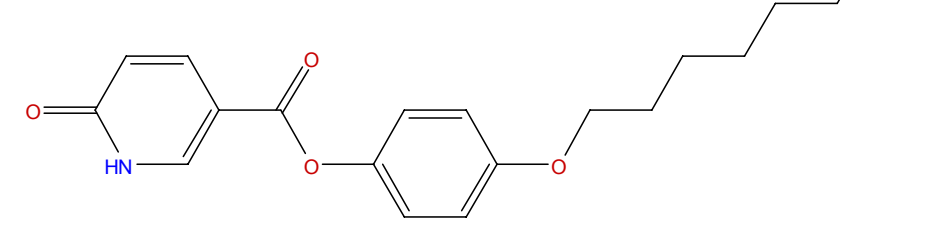

molecular

329.40

weight (Da)

multicomponent no

Fritz, J., Probst, O., Dey, S., Grafström, S., Kowalski, J. \& Neumann, R. Surf. Sci. 1995 329, L613-L618.

\section{Record 263}

4-(((2S,3S)-3-propyloxiran-2-yl)methoxy)phenyl 4((S)-3,7-dimethyloctyloxy)benzoate (MDW 74)

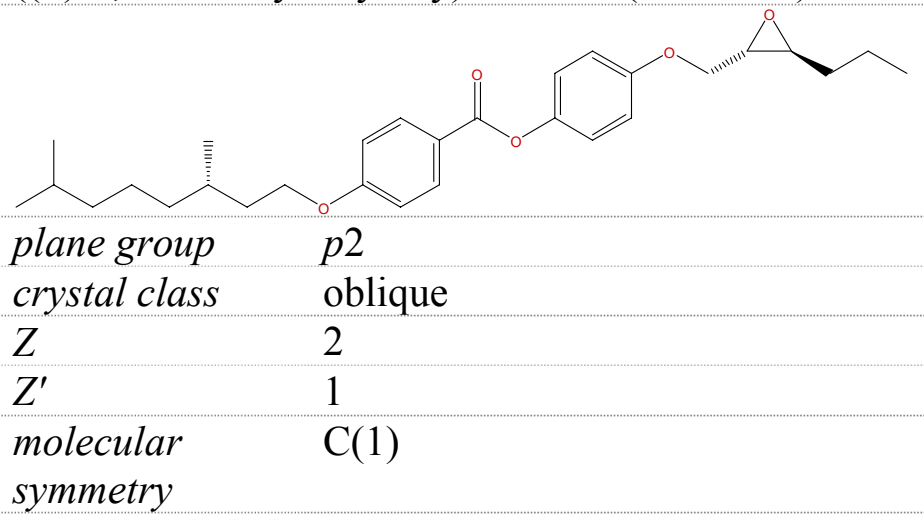

\begin{tabular}{ll} 
plane group & $p 2$ \\
crystal class & oblique \\
\hline$Z$ & 2 \\
\hline$Z^{\prime}$ & 1
\end{tabular}

molecular $\quad \mathrm{C}(\mathrm{s})$

symmetry

site symmetry 1

molecular $\quad 343.42$

weight (Da)

multicomponent no

Heinz, R., Rabe, J. P., Meister, W.-V. \& Hoffmann, S. "Structure and Dynamics of Two-Dimensional Adlayers of a 2-Pyridone Smectogen Studied by STM." Thin Solid Films 1995 264, 246-249.

\section{Record 262}

4-[4-(n,N-dimethylamino)phenylazo]azobenzene

(D2)

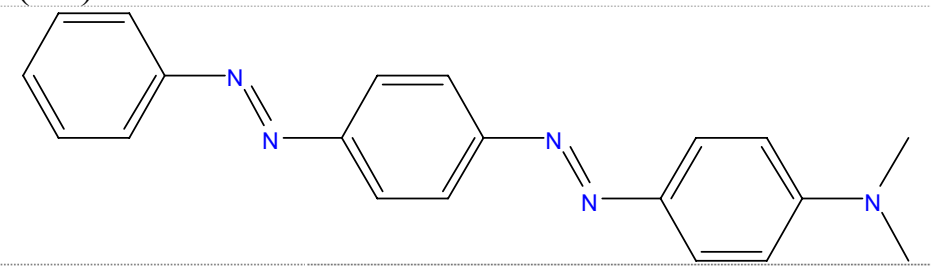

plane group $\quad p 2$

crystal class oblique

$Z \quad 2$

$Z^{\prime} \quad 1$

molecular $\quad \mathrm{C}(\mathrm{s})$

symmetry

site symmetry 1 site symmetry 1

molecular $\quad 468.62$

weight (Da)

multicomponent no

Walba, D. M., Stevens, F., Parks, D. C., Clark, N. A. \& Wand, M. D. Science 1995 267, 1144-1147.

\section{Record 264}

2-Hexadecylanthraquinone (3)

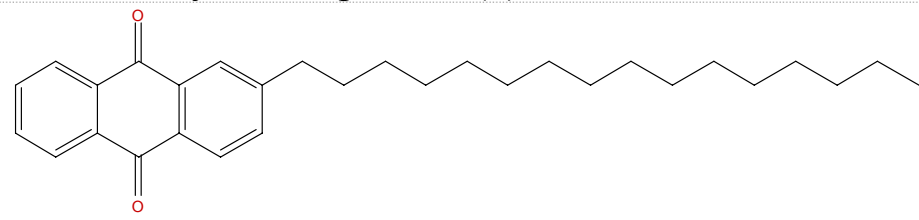

plane group $\quad p 2$

crystal class oblique

Z 2

$Z^{\prime} \quad 1$

molecular $\quad \mathrm{C}(\mathrm{s})$

symmetry

site symmetry 1

molecular $\quad 432.64$

weight (Da)

multicomponent no

Stabel, A., Heinz, R., Rabe, J. P., Wegner, G., De Schryver, F. C., Corens, D., Dehaen, W. \& Süling, C. "STM Investigation of 2D Crystals of Anthrone Derivatives on Graphite - Analysis of MolecularStructure and Dynamics." J. Phys. Chem. 1995 99, 8690-8697. 
Record 265

2-Hexadecylanthraquinone (3)

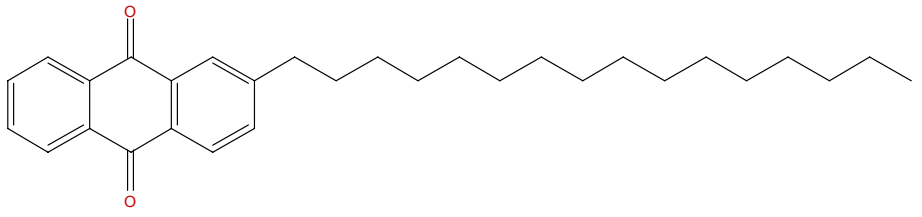

\begin{tabular}{ll} 
plane group & $p 2$ \\
crystal class & oblique \\
\hline$Z$ & 2 \\
\hline$Z^{\prime}$ & 1 \\
molecular & $\mathrm{C}(\mathrm{s})$ \\
symmetry & \\
site symmetry & 1 \\
$\begin{array}{l}\text { molecular } \\
\text { weight }(\mathrm{Da})\end{array}$ & 432.64 \\
multicomponent & no \\
\hline \begin{tabular}{l} 
Stabel, A \\
\hline
\end{tabular}
\end{tabular}

Stabel, A., Heinz, R., Rabe, J. P., Wegner, G., De Schryver, F. C., Corens, D., Dehaen, W. \& Süling, C. J. Phys. Chem. 1995 99, 8690-8697.

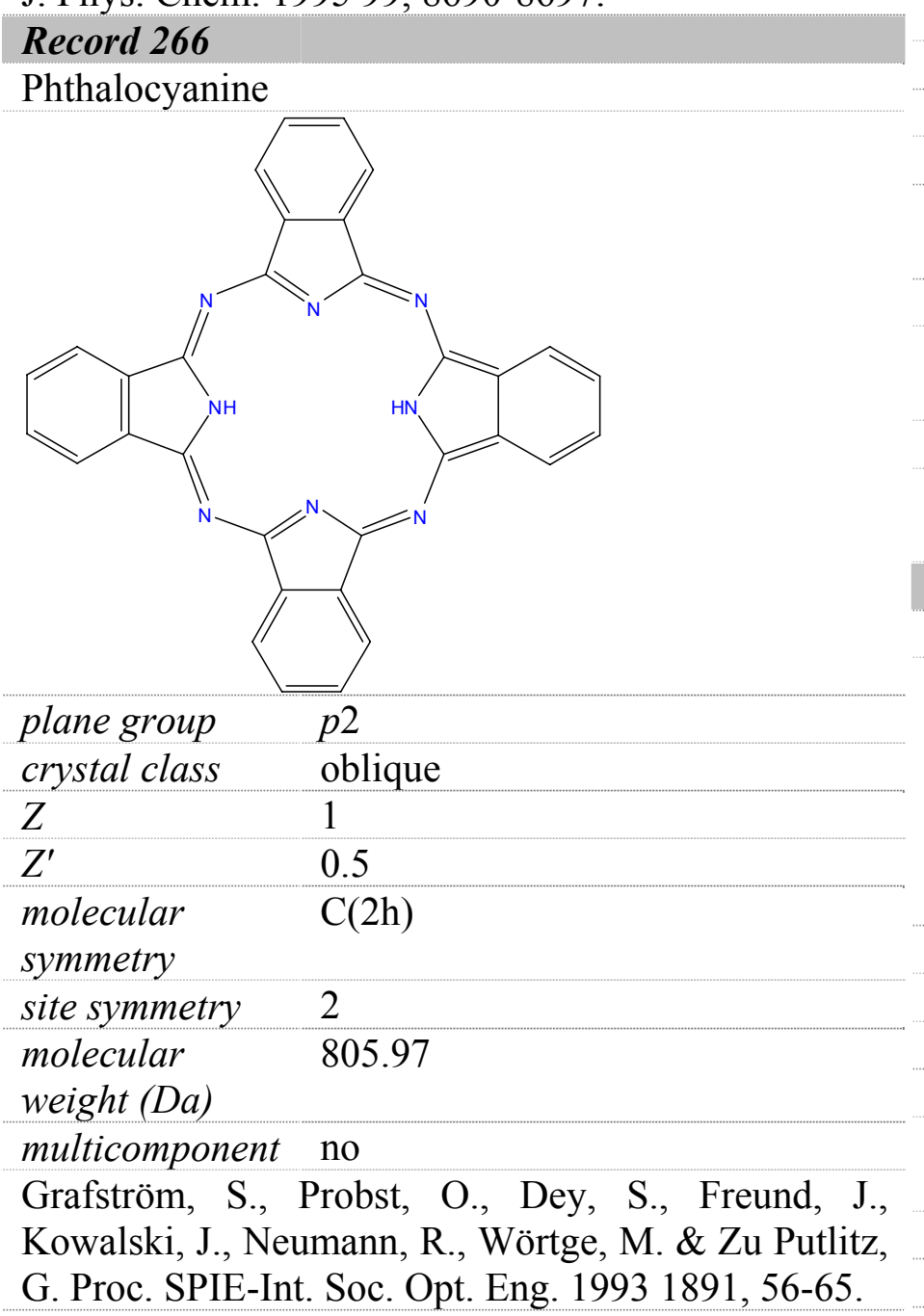

\section{Record 267}

hexakis-2,3,6,7,10,11-heptyloxytriphenylene

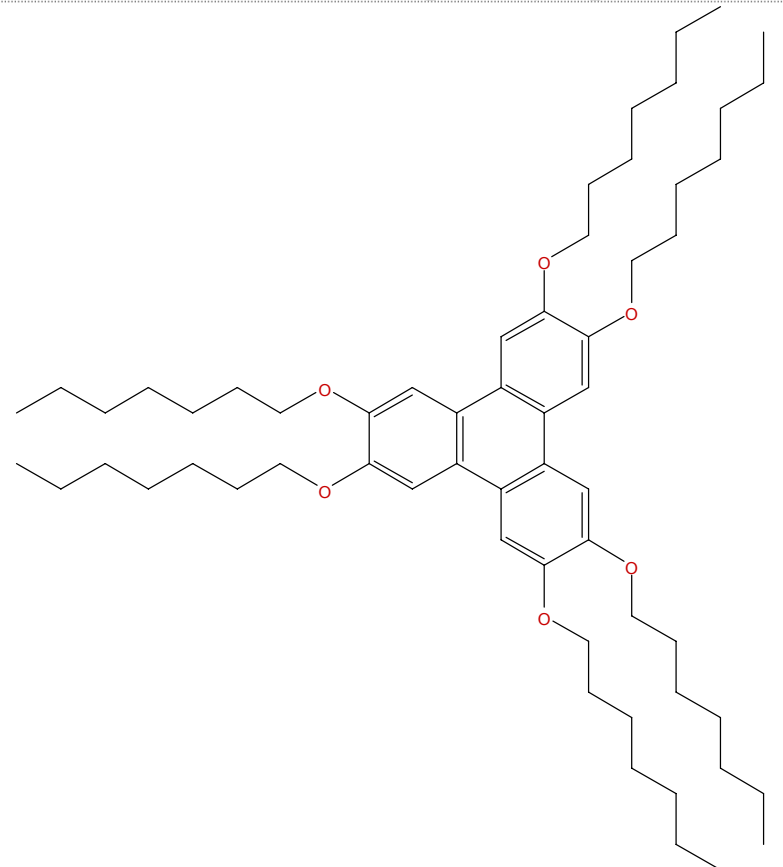

plane group $\quad$ p2

crystal class oblique

$\begin{array}{ll}Z & 2 \\ Z^{\prime} & 1 \\ \text { molecular } & \mathrm{D}(3 \mathrm{~h}) \\ \text { symmetry } & \\ \text { site symmetry } & 1 \\ \text { molecular } & 913.40\end{array}$

weight (Da)

multicomponent no

Askadskaya, L., Boeffel, C. \& Rabe, J. P. Ber. Bunsen.-Ges. 1993 97, 517-521.

\section{Record 268}

2-fluorooctadecanoic acid
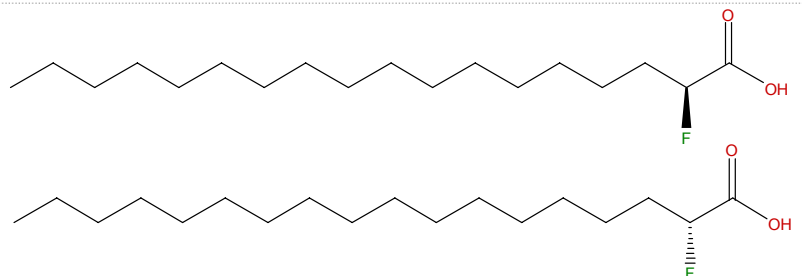

plane group $\quad$ p2

crystal class oblique

$\begin{array}{ll}Z & 2 \\ Z^{\prime} & 1\end{array}$

molecular $\quad \mathrm{C}(1)$

symmetry

site symmetry $\quad 1$

molecular $\quad 604.94$ 
weight (Da)

multicomponent yes

Stabel, A., Dasaradhi, L., O'Hagan, D. \& Rabe, J. P. Langmuir 1995 11, 1427-1430.

\section{Record 269}

5-(octadecyloxy)isophthalic acid coadsorbed with octanol
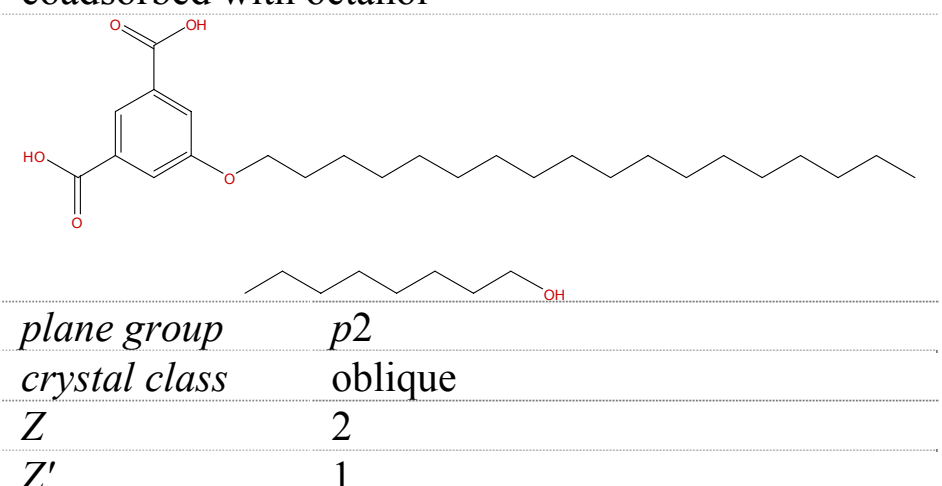

\section{molecular $\mathrm{C}(\mathrm{s}), \mathrm{C}(\mathrm{s})$}

symmetry

$\begin{array}{ll}\text { site symmetry } & 11 \\ \text { molecular } & 564.84\end{array}$

weight (Da)

multicomponent yes

Vanoppen, P., Grim, P. C. M., Rücker, M., De Feyter, S., Moessner, G., Valiyaveettil, S., Müllen, K. \& De Schryver, F. C. J. Phys. Chem. 1996 100, 19636-19641.

\section{Record 270}

5-[10-(2-methylbutoxy)-decyloxy]isophthalic acid with heptanol

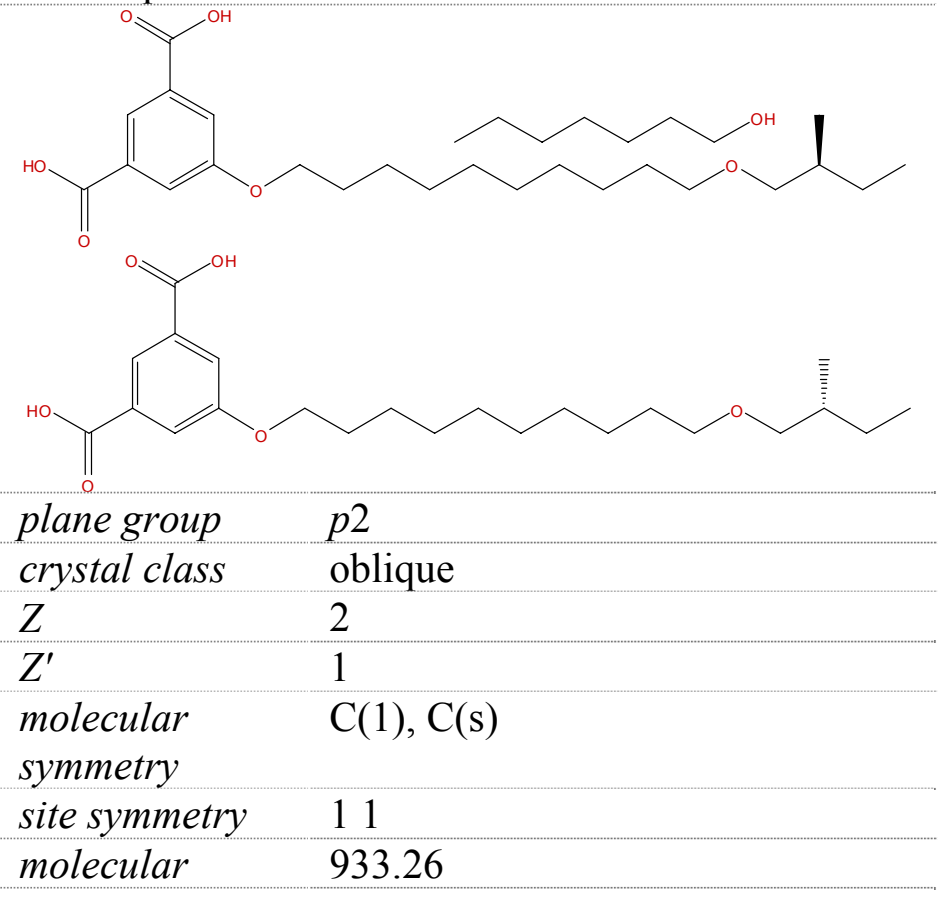

weight (Da)

multicomponent yes

De Feyter, S., Grim, P. C. M., Rücker, M., Vanoppen, P., Meiners, C., Sieffert, M., Valiyaveettil, S., Müllen, K. \& De Schryver, F. C. Angew. Chem., Int. Edit. 1998 37, 1223-1226.

Record 271

S-1,2-dihydroxyoctadecane

$\begin{array}{ll}\text { plane group } & p 2 \\ \text { crystal class } & \text { oblique } \\ Z & 2 \\ Z^{\prime} & 1 \\ \text { molecular } & \mathrm{C}(1) \\ \text { symmetry } & 1 \\ \text { site symmetry } & 1 \\ \text { molecular } & 286.49 \\ \text { weight (Da) } & \\ \text { multicomponent } & \text { no } \\ \text { Qian, P., Nanjo, H., Yokoyama, T. \& Suzuki, T. M. } \\ \text { Chem. Lett. 1998 1133-1134. } \\ \text { Record 272 }\end{array}$

R-5-[10-(2-methylbutoxy)-decyloxy]isophthalic acid with heptanol

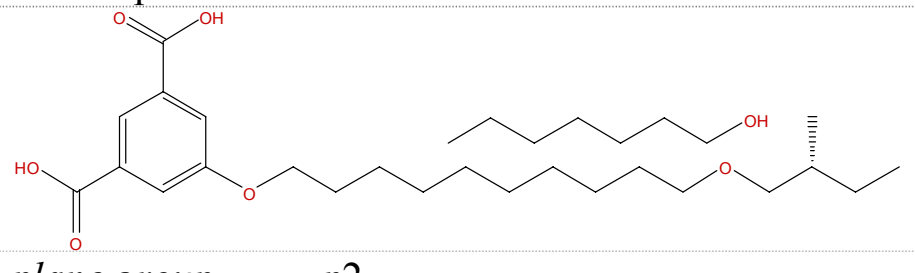

plane group $\quad$ p2

crystal class oblique

$Z \quad 2$

$Z^{\prime} \quad 1$

molecular $\mathrm{C}(1), \mathrm{C}(\mathrm{s})$

symmetry

site symmetry 11

molecular $\quad 524.73$

weight (Da)

multicomponent yes

De Feyter, S., Grim, P. C. M., Rücker, M., Vanoppen, P., Meiners, C., Sieffert, M., Valiyaveettil, S., Müllen, K. \& De Schryver, F. C. Angew. Chem., Int. Edit. 1998 37, 1223-1226.

\section{Record 273}

(S)-2-nitro-4-(octan-2-yloxy)phenyl 


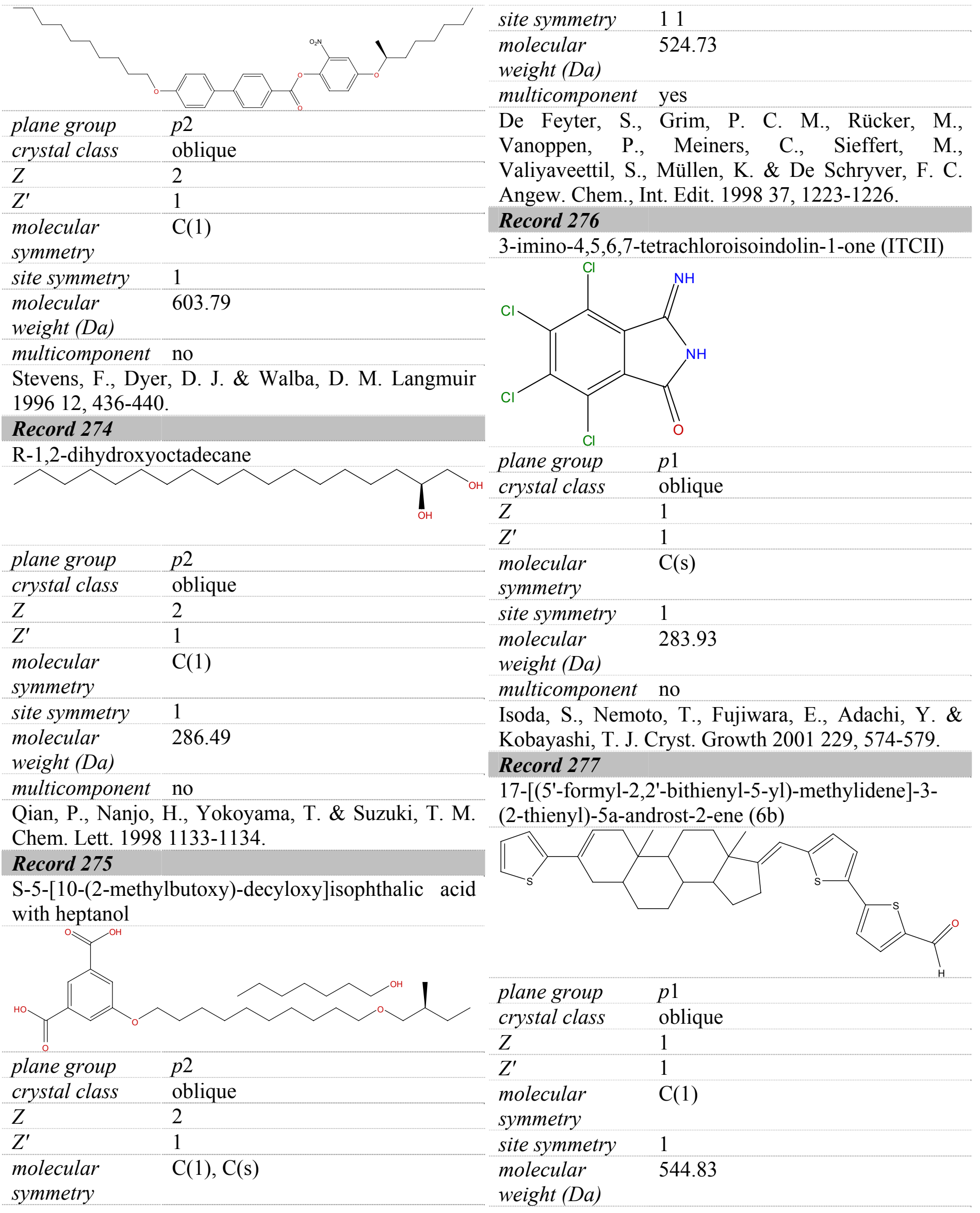


multicomponent no

Vollmer, M. S., Effenberger, F., Stecher, R., Gompf, B. \& Eisenmenger, W. Chem.-Eur. J. 1999 5, 96-101.

\section{Record 278}

5-nonadecyl-2-(5-nonadecylpyridin-2-yl)pyridine

\begin{tabular}{|c|c|}
\hline plane group & $p 1$ \\
\hline crystal class & oblique \\
\hline$Z$ & 1 \\
\hline$Z^{\prime}$ & 1 \\
\hline $\begin{array}{l}\text { molecular } \\
\text { symmetry }\end{array}$ & $C(2 v)$ \\
\hline site symmetry & 1 \\
\hline $\begin{array}{l}\text { molecular } \\
\text { weight (Da) }\end{array}$ & 823.65 \\
\hline multicomponent & yes \\
\hline
\end{tabular}

Schuurmans, N., Verkuijl, B. J. V., Van Esch, J. H., Feringa, B. L. \& De Schryver, F. C. Chem.-Eur. J. 2004 10, 1124-1132.

\section{Record 279}

5-nonadecyl-2-(5-nonadecylpyridin-2-yl)pyridine

$\begin{array}{ll}\text { plane group } & p 1 \\ \text { crystal class } & \text { oblique } \\ Z & 1 \\ Z^{\prime} & 1 \\ \text { molecular } & \mathrm{C}(2 \mathrm{v})\end{array}$

symmetry

site symmetry 1

molecular $\quad 870.83$

weight (Da)

multicomponent yes

De Feyter, S., Abdel-Mottaleb, M. M. S., Schuurmans, N., Verkuijl, B. J. V., Van Esch, J. H., Feringa, B. L. \& De Schryver, F. C. Chem.-Eur. J. 2004 10, 1124-1132.

\section{Record 280}

5-methyl-2-(5-nonadecylpyridin-2-yl)pyridine

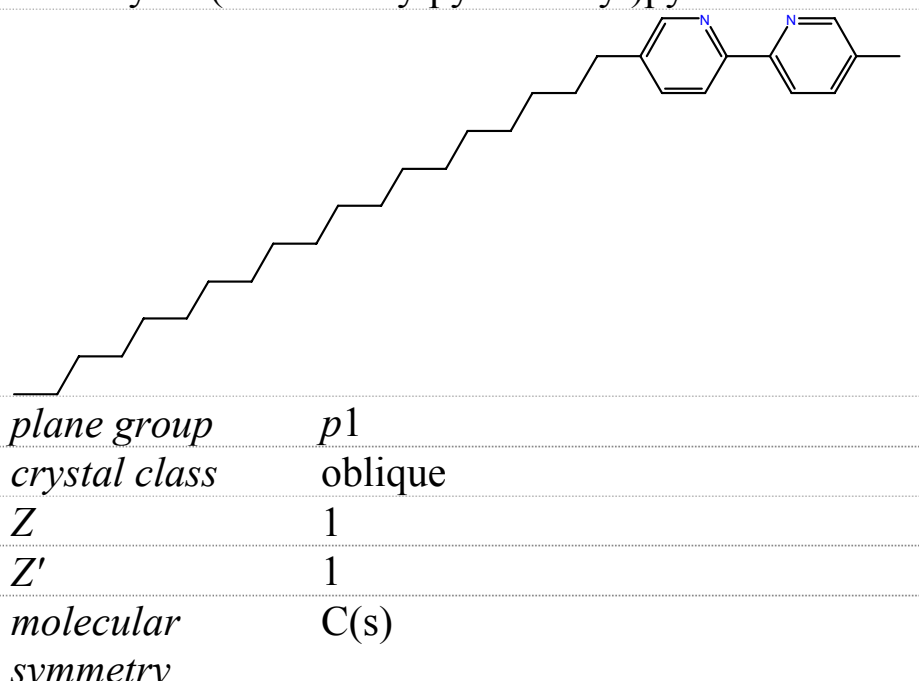

symmetry

site symmetry 1

molecular $\quad 436.72$

weight (Da)

multicomponent no

De Feyter, S.; Abdel-Mottaleb, M. M. S.; Schuurmans, N.; Verkuijl, B. J. V.; Van Esch, J. H.; Feringa, B. L.; De Schryver, F. C. Chem.-Eur. J. 2004, 10, 1124-1132.

\section{Record 281}

4-n-octyl-4'-cyanobiphenyl (8CB)

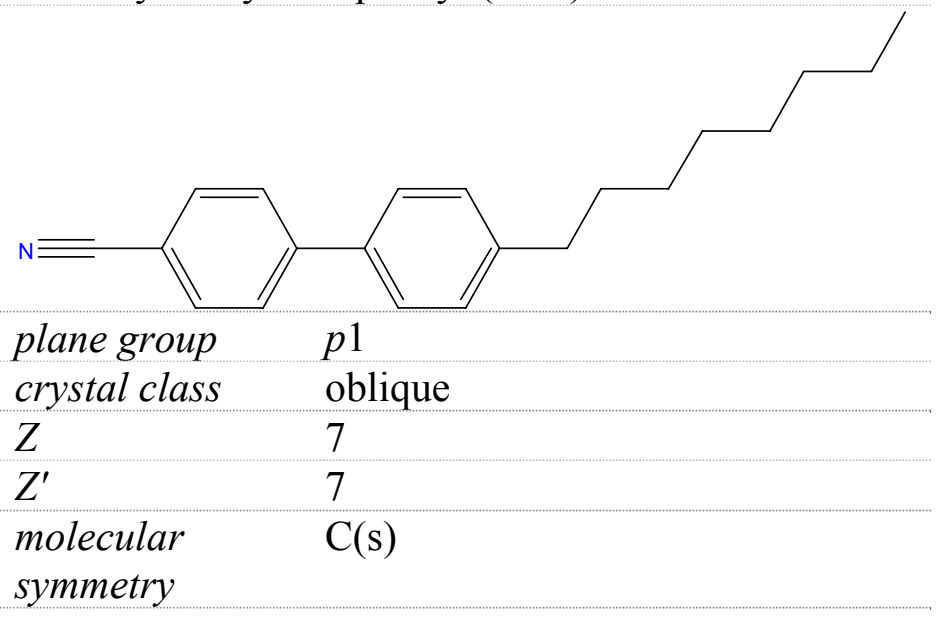


site symmetry $\quad 1111111$

molecular

291.43

weight (Da)

multicomponent no

Mcmaster, T. J., Carr, H., Miles, M. J., Cairns, P. \& Morris, V. J. Liq. Cryst. 1991 9, 11-18.

\section{Record 282}

iodo-phenyloctadecyl ether

plane group
crystal class $\quad$ oblique
$Z$

\section{Record 283}

17,18-pentatriacontadiene

$\begin{array}{ll}\text { plane group } \quad p 1 \\ \text { crystal class } & \text { oblique } \\ Z & 1 \\ Z^{\prime} & 1 \\ \text { molecular } & \mathrm{C}(2 \mathrm{v}) \\ \text { symmetry } & 1 \\ \text { site symmetry } & 1 \\ \text { molecular } & 488.91 \\ \text { weight (Da) } & \\ \text { multicomponent } & \text { no } \\ \text { Claypool, C. L., Faglioni, F., Goddard, W. A. \& } \\ \text { Lewis, N. S. J. Phys. Chem. B 1999 103, 7077-7080. } \\ \text { Record 284 }\end{array}$

\section{Record 284}

1-tetracosanol

$\begin{array}{ll}\text { plane group } & p 1 \\ \text { crystal class } & \text { oblique } \\ Z & 2 \\ Z^{\prime} & 2 \\ \text { molecular } & \mathrm{C}(\mathrm{s})\end{array}$

symmetry

site symmetry 11

molecular $\quad 354.65$

weight (Da)

multicomponent no

Buchholz, S. \& Rabe, J. P. Angew. Chem., Int. Edit. 1992 31, 189-191.

\section{Record 285}

2-bromo-5,8,11,14,17-pentadodecylhexa-peri-hexabenzocoronene $(2 \mathrm{a})$ 
molecular

186.33

weight (Da)

multicomponent no

Yeo, Y. H., McGonigal, G. C. \& Thomson, D. J. Langmuir 1993 9, 649-651.

\section{Record 287}

hexakis(zinc (II) porphyrinato)benzene (Zn1) and DABCO

\begin{tabular}{|c|c|}
\hline plane group & $p 1$ \\
\hline crystal class & oblique \\
\hline$Z$ & 1 \\
\hline$Z^{\prime}$ & 1 \\
\hline $\begin{array}{l}\text { molecular } \\
\text { symmetry }\end{array}$ & $\mathrm{S}(6), \mathrm{D}(3 \mathrm{~d})$ \\
\hline site symmetry & 11 \\
\hline $\begin{array}{l}\text { molecular } \\
\text { weight (Da) }\end{array}$ & 8834.86 \\
\hline
\end{tabular}

Elemans, J. A. A. W., Lensen, M. C., Gerritsen, J. W., van Kempen, H., Speller, S., nolte, R. J. M. \& Rowan, A. E. Adv. Mater. 2003 15, 2070-2073.

\section{Record 288}

1,1-diethoxy-3-imino-4,5,6,7-tetrachloroisoindoline (DEITCI)

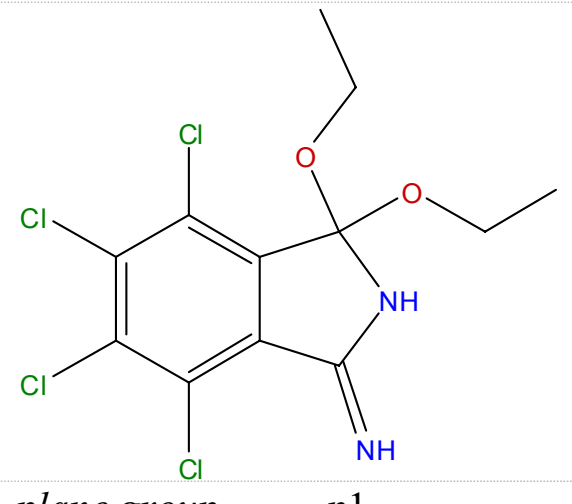

plane group $\quad p 1$

crystal class oblique

$Z \quad 1$

$Z^{\prime} \quad 1$

molecular $\mathrm{C}(\mathrm{s})$

symmetry

site symmetry 1

molecular $\quad 358.05$

weight (Da)

multicomponent no

Nemoto, T., Adachi, Y., Fujiwara, E. \& Isoda, S.

Mol. Cryst. Liq. Cryst. Sci. Technol., Sect. A 2002 389, 97-104.

\section{Record 289}

$\mathrm{Ru} 7$

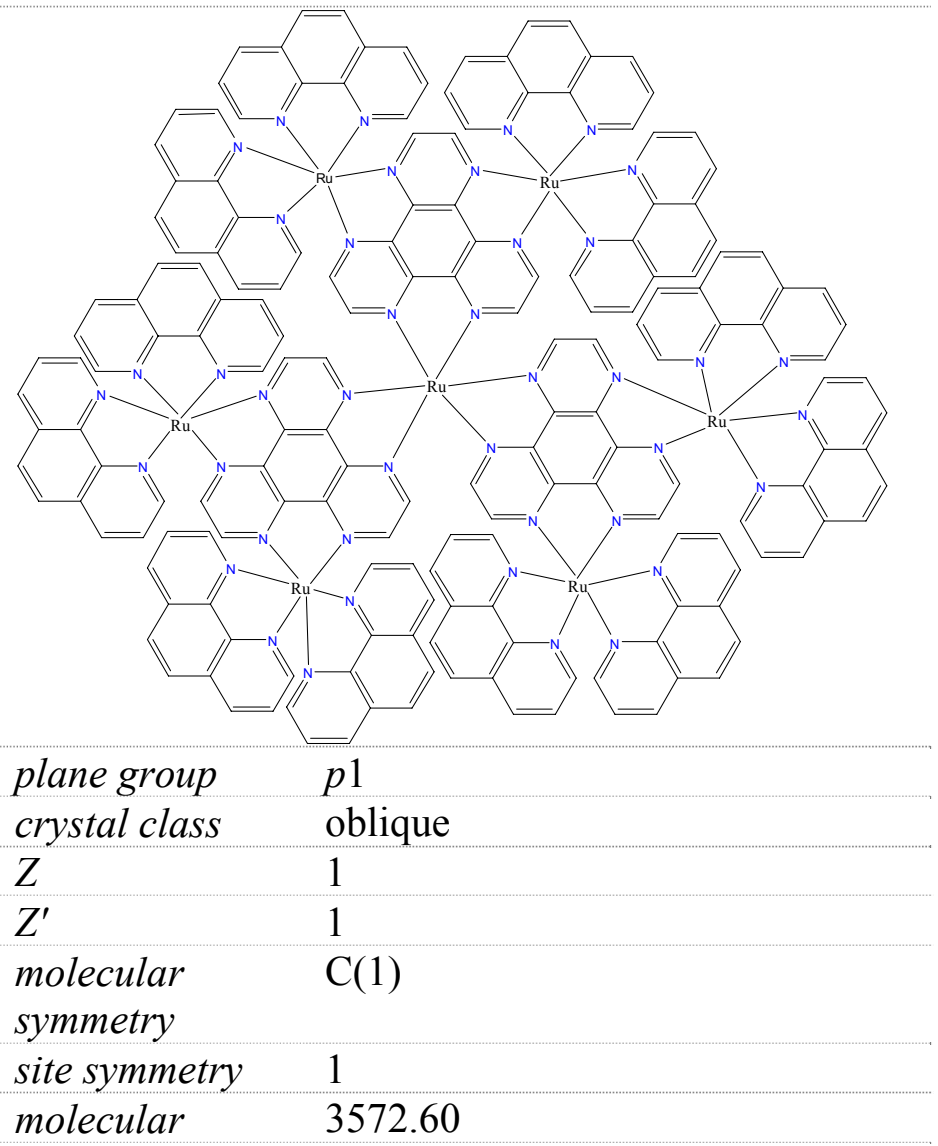


weight (Da)

multicomponent no

Latterini, L., Pourtois, G., Moucheron, C., Lazzaroni,

R., Brédas, J.-L., Mesmaeker, A. K.-D. \& De

Schryver, F. C. Chem.-Eur. J. 2000 6, 1331-1336.

\section{Record 290}

n-octadecylacrylamide

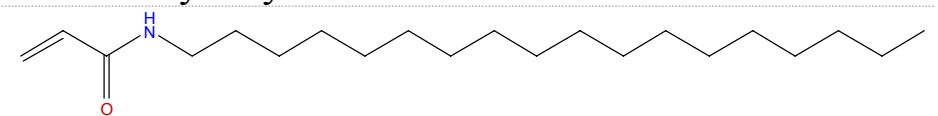

plane group $\quad p 1$

crystal class oblique

Z 1

$Z^{\prime} \quad 1$

molecular

$\mathrm{C}(\mathrm{s})$

symmetry

site symmetry 1

molecular $\quad 323.56$

weight (Da)

multicomponent no

Qian, P., Nanjo, H., Yokoyama, T., Suzuki, T. M. \&

Miyashita, T. Chem. Commun. 1998 943-944.

\section{Record 291}

11,11',11",11"',11'"',11"'"--

(hexabenzo[bc,ef,hi,kl,no,qr]coronene-

2,5,8,11,14,17-hexayl)hexakis(undecane-11,1-diyl)

hexakis(9,10-dioxo-9,10-dihydroanthracene-2-

carboxylate)

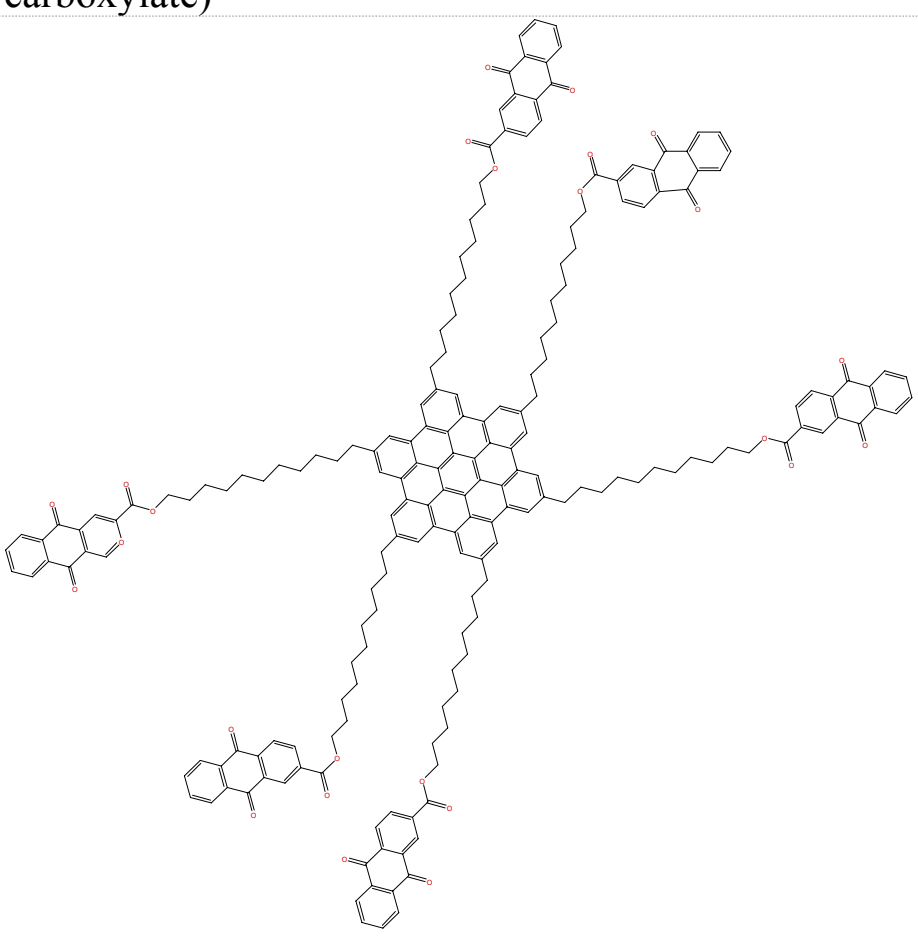

plane group $\quad p 1$

crystal class oblique

Z 1

$Z^{\prime} \quad 1$

molecular $\quad \mathrm{C}(6 \mathrm{~h})$

symmetry

site symmetry 1

molecular $\quad 2949.58$

weight (Da)

multicomponent no

Jäckel, F., Yin, X., Samorí, P., Tchebotareva, N., Watson, M. D., Venturini, A., Müllen, K. \& Rabe, J. P. Synth. Met. 2004 147, 5-9.

\section{Record 292}

Ester-23; 1,3-di-(1-heneicosyl) isophthalate

$\begin{array}{ll}\text { plane group } & p 1 \\ \text { crystal class } & \text { oblique }\end{array}$

$Z$ 5

$Z^{\prime} \quad 5$

molecular $\quad \mathrm{C}(2 \mathrm{v})$

symmetry

site symmetry $\quad 11111$

molecular $\quad 755.25$

weight (Da)

multicomponent no

Plass, K. E., Kim, K. \& Matzger, A. J. J. Am. Chem.

Soc. 2004 126, 9042-9053.

\section{Record 293}

octadecamine

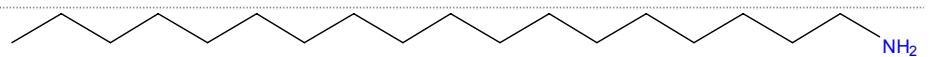

plane group $\quad p 1$

crystal class oblique

$Z \quad 2$

$Z^{\prime} \quad 2$

molecular $\quad \mathrm{C}(\mathrm{s})$

symmetry

site symmetry 11

molecular $\quad 269.51$

weight $(D a)$

multicomponent no

Cyr, D. M., Venkataraman, B., Flynn, G. W., Black,

A. \& Whitesides, G. M. J. Phys. Chem. 1996 100, $13747-13759$. 


\section{Record 294}

2-Hydroxymethyl-[1,4,7,10,13-

pentaoxabenzocyclopentadecane]-3,4,5-tris[4(dodecyl-1-oxy)benzyloxy]benzoate (2),

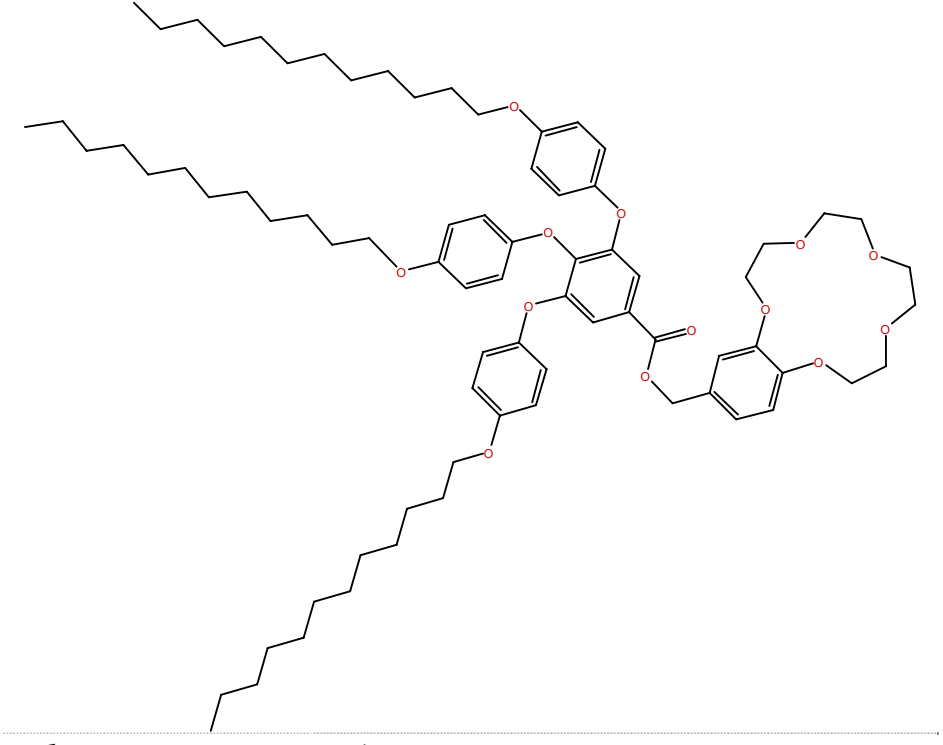

plane group $\quad p 1$

crystal class oblique

$\begin{array}{ll}Z & 1 \\ Z^{\prime} & 1\end{array}$

molecular $\mathrm{C}(\mathrm{s})$

symmetry

site symmetry 1

molecular $\quad 1231.68$

weight (Da)

multicomponent no

Mourran, A., Beginn, U., Zipp, G. \& Möller, M. Langmuir 2004 20, 673-679.

\section{Record 295}

1,3-di(octadec-1-ynyl)benzene (Alkyne-18)

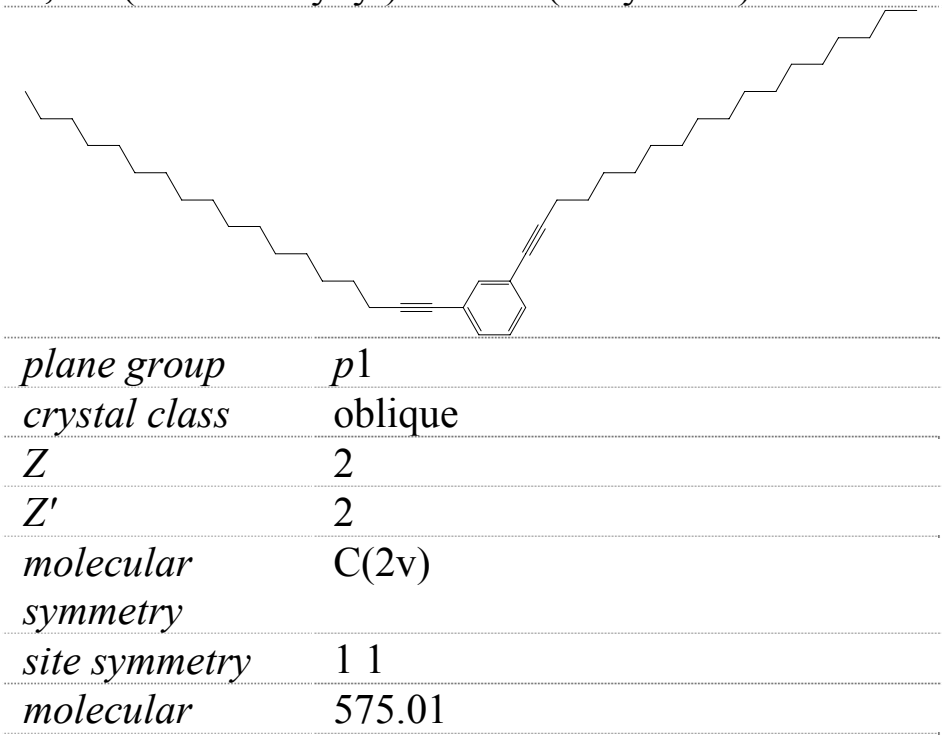

weight (Da)

multicomponent no

Plass, K. E., Kim, K. \& Matzger, A. J. J. Am. Chem.

Soc. 2004 126, 9042-9053.

\section{Record 296}

N-nonadecyl-benzamide NDB

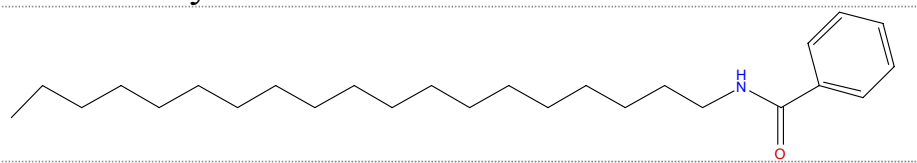

plane group $\quad p 1$

crystal class oblique

$Z+1$

$Z^{\prime} \quad 1$

molecular $\mathrm{C}(\mathrm{s})$

symmetry

site symmetry 1

molecular $\quad 387.64$

weight (Da)

multicomponent no

Nanjo, H., Qian, P., Yokoyama, T. \& Suzuki, T. M.

Jpn. J. Appl. Phys., Part 12003 42, 6560-6563.

\section{Record 297}

octadecyl

2-(2-(tert-

butoxycarbonylamino)propanamido)propanoate

(Boc-Ala-Ala-OC18)

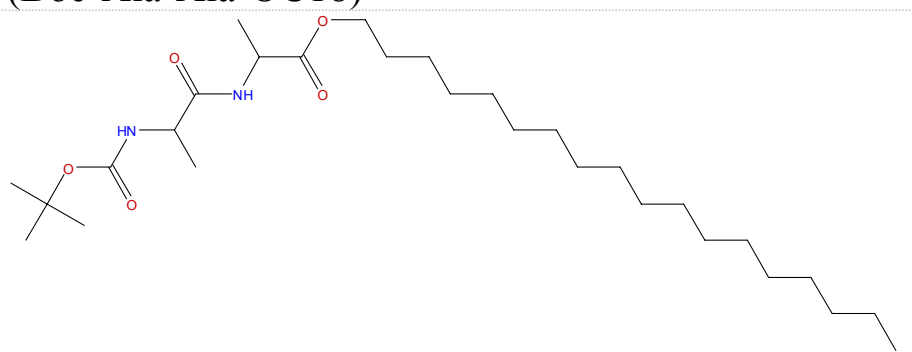

plane group $\quad p 1$

crystal class oblique

$Z \quad 2$

$Z^{\prime} \quad 2$

molecular $\quad \mathrm{C}(1)$

symmetry

site symmetry 11

molecular $\quad 512.77$

weight (Da)

multicomponent no

Hoeppener, S., Chi, L. F., Wonnemann, J., Erker, G.

\& Fuchs, H. Surf. Sci. 2001 487, 9-14.

\section{Record 298}

2-Hexadecylanthrone (1) 


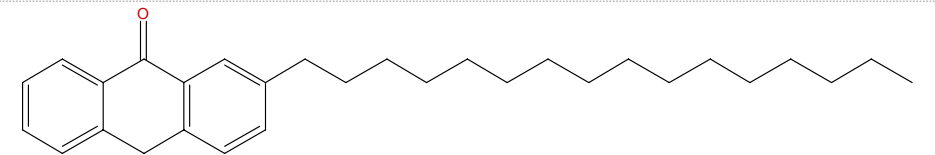

plane group $\quad p 1$

crystal class oblique

$\begin{array}{ll}Z & 1 \\ Z^{\prime} & 1\end{array}$

molecular $\mathrm{C}(\mathrm{s})$

symmetry

site symmetry 1

molecular $\quad 418.65$

weight (Da)

multicomponent no

Stabel, A., Heinz, R., Rabe, J. P., Wegner, G., De

Schryver, F. C., Corens, D., Dehaen, W. \& Süling, C.

J. Phys. Chem. 1995 99, 8690-8697.

\section{Record 299}

(9,10-dimethoxy-9,10-dihydroanthracen-2-yl)methyl 4-(5,8,11,14,17-pentakis(3,7-

dimethyloctyl)hexabenzo[bc,ef,hi,kl,no,qr]coronen-2-

yl)butanoate

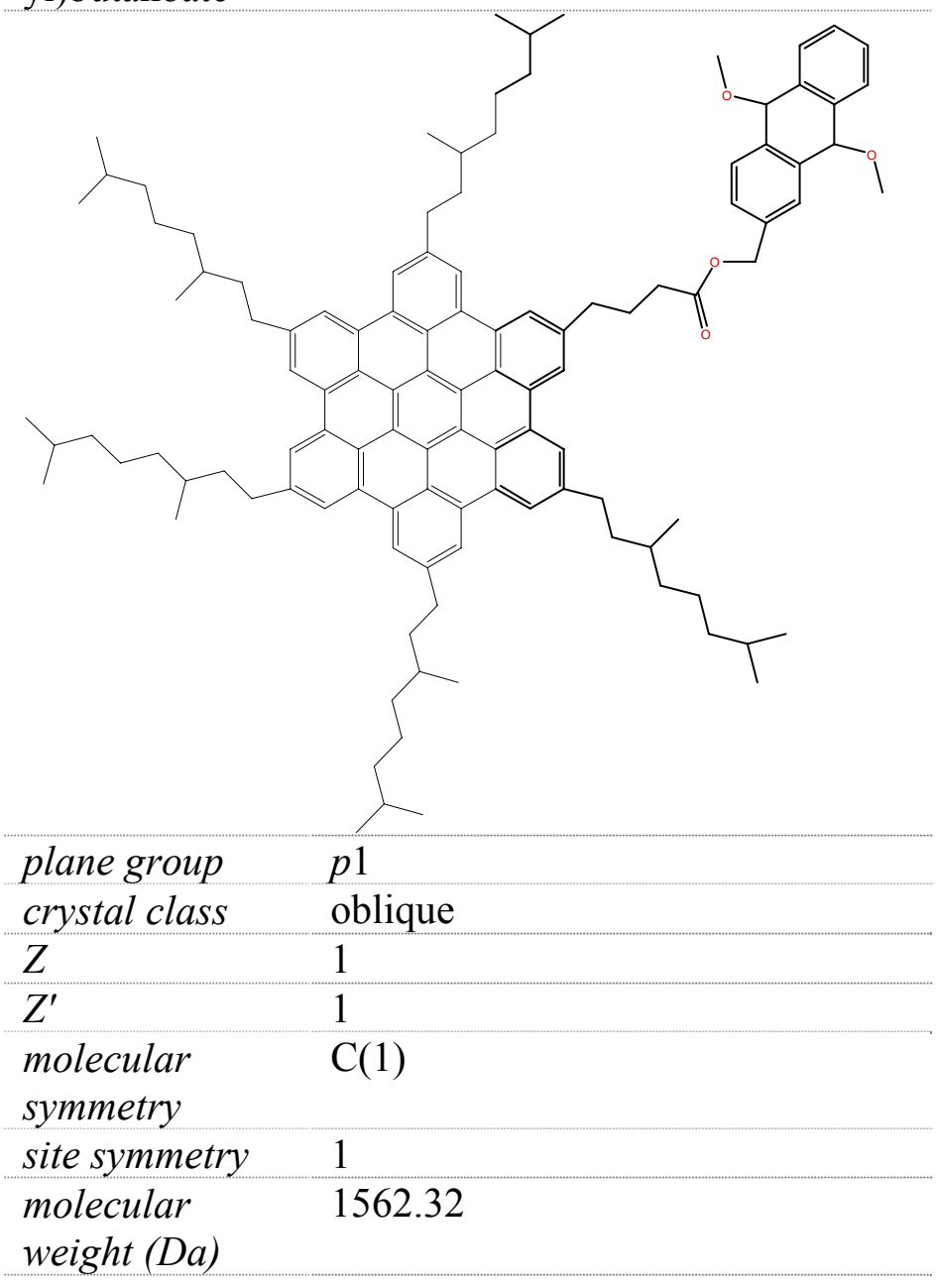

multicomponent no

Samorì, P., Yin, X., Tchebotareva, N., Wang, Z., Pakula, T., Jäckel, F., Watson, M. D., Venturini, A., Müllen, K. \& Rabe, J. P. J. Am. Chem. Soc. 2004 126, 3567-3575.

\section{Record 300}

octadecyl 2-(tert-butoxycarbonylamino)propanoate (Boc-Ala-OC18)

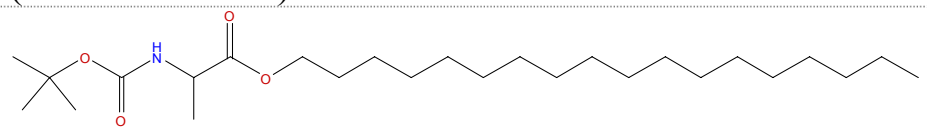

plane group $\quad p 1$

crystal class oblique

$Z \quad 2$

$Z^{\prime} \quad 2$

molecular $\quad \mathrm{C}(1)$

symmetry

site symmetry 11

molecular $\quad 441.69$

weight (Da)

multicomponent no

Hoeppener, S., Chi, L. F., Wonnemann, J., Erker, G. \& Fuchs, H. Surf. Sci. 2001 487, 9-14.

\section{Record 301}

(E)-octadecyl 3-(4-(octadecyloxy)phenyl)acrylate (C18CinnC18)

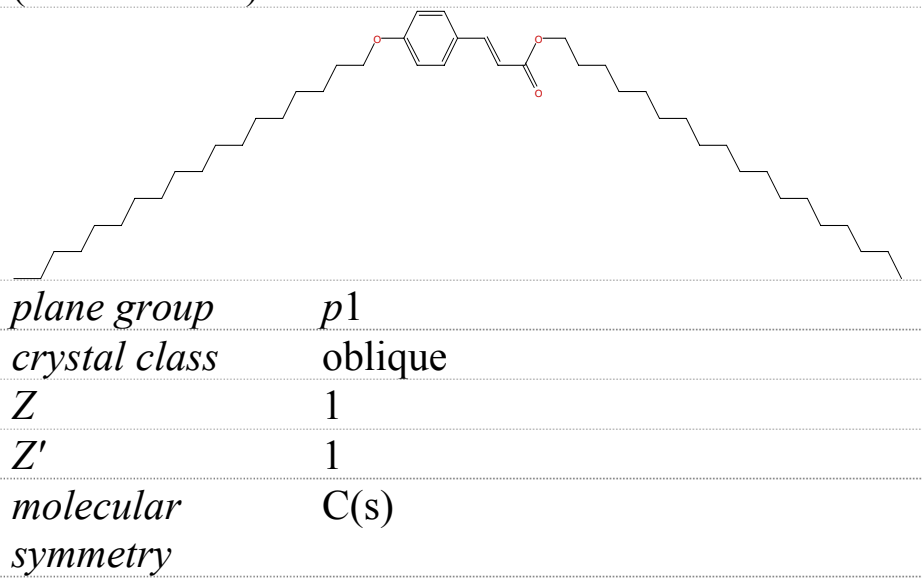
site symmetry 1

molecular $\quad 669.11$

weight (Da)

multicomponent no

Abdel-Mottaleb, M. M. S., De Feyter, S., Gesquière, A., Sieffert, M., Klapper, M., Müllen, K. \& De Schryver, F. C. Nano Lett. 2001 1, 353-359.

Record 302

1,10-dihexadecoxy decane C16OC100C16 


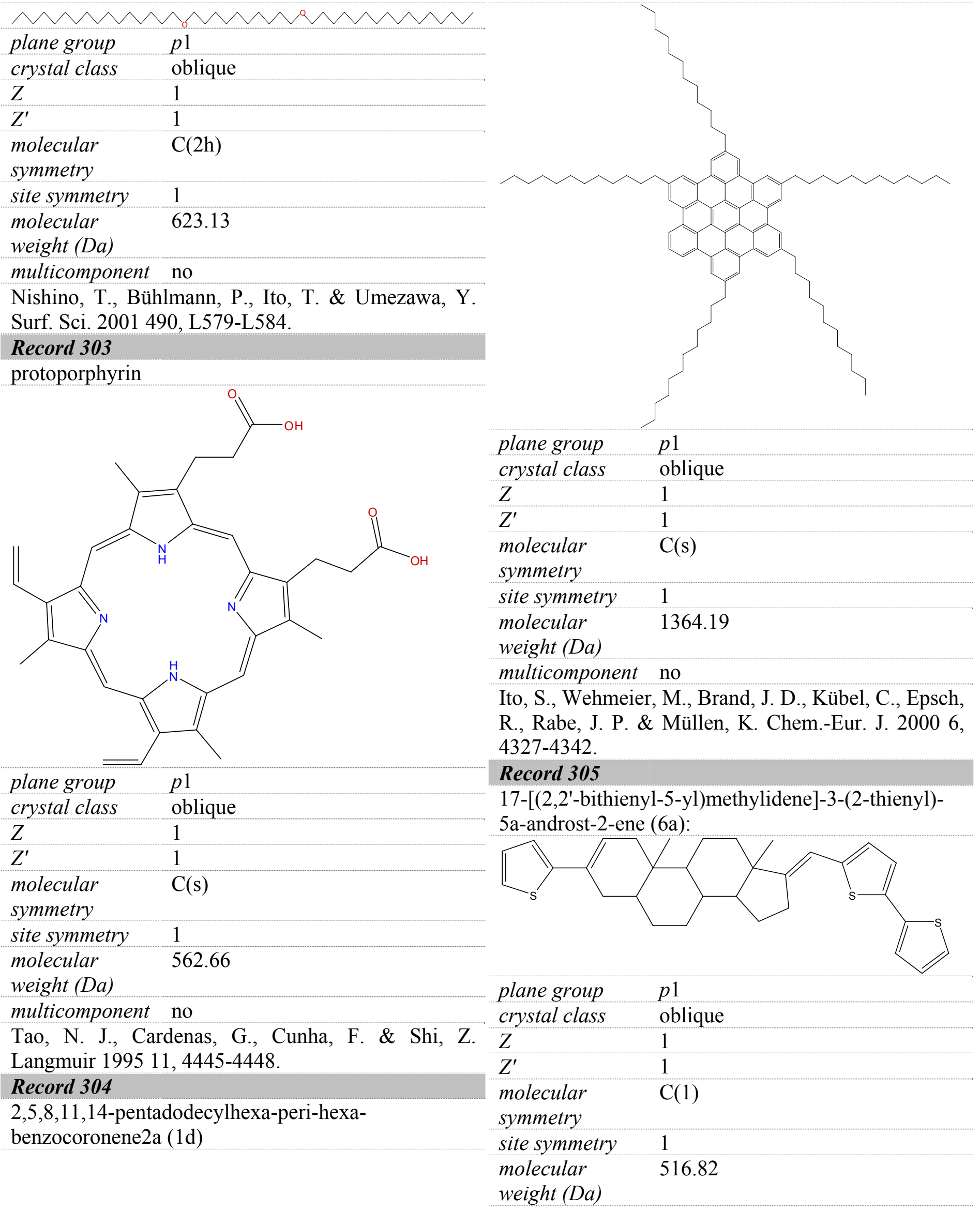


multicomponent no

Vollmer, M. S., Effenberger, F., Stecher, R., Gompf, B. \& Eisenmenger, W. Chem.-Eur. J. 1999 5, 96-101.

\section{Record 306}

1-tetracosanol

Planegrat

\begin{tabular}{|c|c|}
\hline plane group & $p 1$ \\
\hline crystal class & oblique \\
\hline$Z$ & 2 \\
\hline$Z^{\prime}$ & 2 \\
\hline $\begin{array}{l}\text { molecular } \\
\text { symmetry }\end{array}$ & $\mathrm{C}(\mathrm{s})$ \\
\hline site symmetry & 11 \\
\hline $\begin{array}{l}\text { molecular } \\
\text { weight }(D a)\end{array}$ & 368.68 \\
\hline multicomponent & no \\
\hline
\end{tabular}

\section{Record 307}

1-dodecyl-2,5-bis(5-(1-octadecyl-5-(thiophen-2-yl)1H-pyrrol-2-yl)thiophen-2-yl)-1H-pyrrole (SN18SN12SN18S)

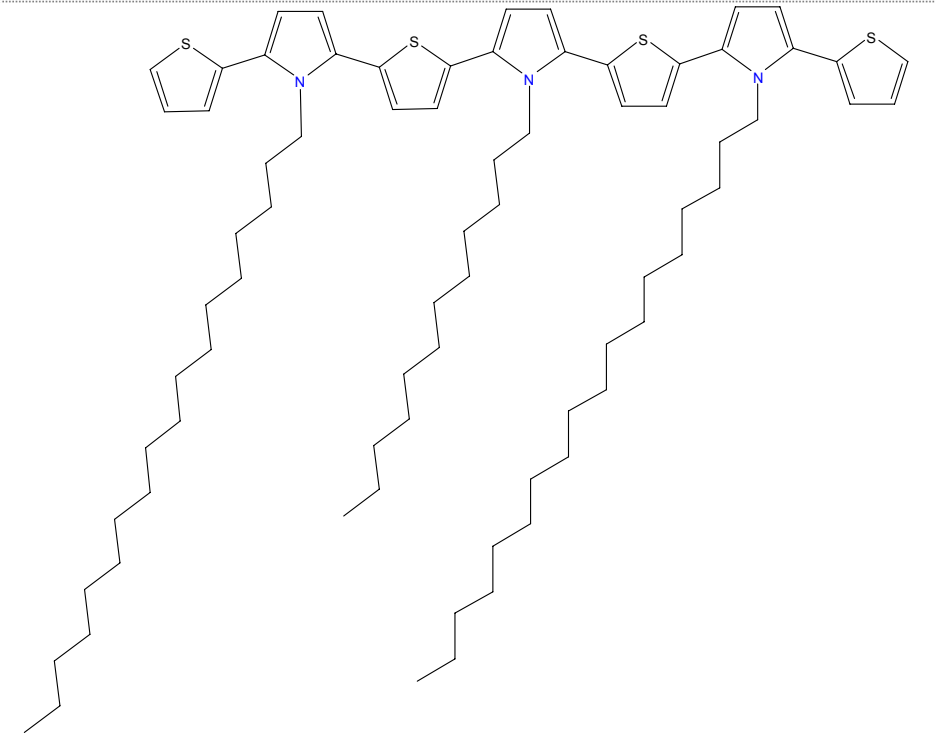

plane group $\quad p 1$

crystal class oblique

$Z$ -

$Z^{\prime} \quad 1$

molecular $\mathrm{C}(\mathrm{s})$

symmetry

site symmetry 1

molecular $\quad 1199.01$

weight (Da)

multicomponent no
Liu, T. L., Parakka, J. P., Cava, M. P. \& Kim, Y. T.

Langmuir 1995 11, 4205-4208.

\section{Record 308}

(2R,3S,5R)-5-(2-amino-6-oxo-1H-purin-9(6H)-yl)-2((4-(hexyloxy)benzoyloxy)methyl)tetrahydrofuran-3yl 4-(hexyloxy)benzoate

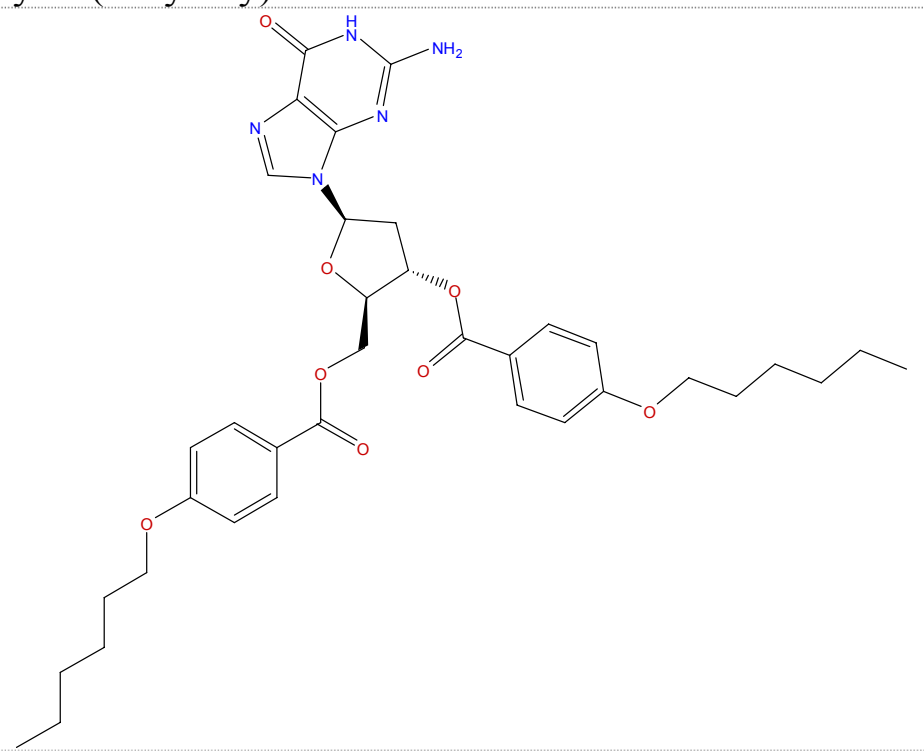

plane group $\quad p 1$

crystal class oblique

$Z \quad 2$

$Z^{\prime} \quad 2$

molecular $\quad \mathrm{C}(1)$

symmetry

site symmetry 1

molecular $\quad 675.77$

weight (Da)

multicomponent no

Gottarelli, G., Masiero, S., Mezzina, E., Pieraccini, S., Rabe, J. P., Samorí, P. \& Spada, G. P. Chem.-Eur. J. 2000 6, 3242-3248.

\section{Record 309}

Alkane-16; 1,3-di-(1-octadecyl) benzene

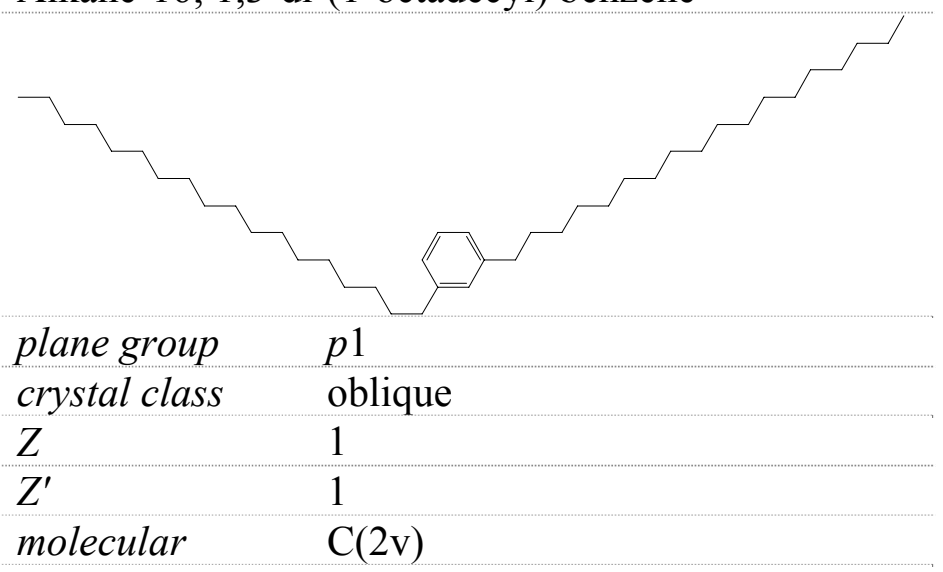


symmetry

site symmetry $\quad 1$

molecular $\quad 583.07$

weight (Da)

multicomponent no

Plass, K. E., Kim, K. \& Matzger, A. J. J. Am. Chem.

Soc. 2004 126, 9042-9053.

\section{Record 310}

17-pentatriacontene

NNMNAN)

plane group $\quad p 1$

crystal class oblique

$Z^{\prime} \quad 1$

molecular $\mathrm{C}(\mathrm{s})$

symmetry

site symmetry 1

molecular $\quad 490.93$

weight (Da)

multicomponent no

Claypool, C. L., Faglioni, F., Goddard, W. A., Gray,

H. B., Lewis, N. S. \& Marcus, R. A. J. Phys. Chem.

B 1997 101, 5978-5995.

\section{Record 311}

$\mathrm{N}$-octadecylbenzamide (ODB)

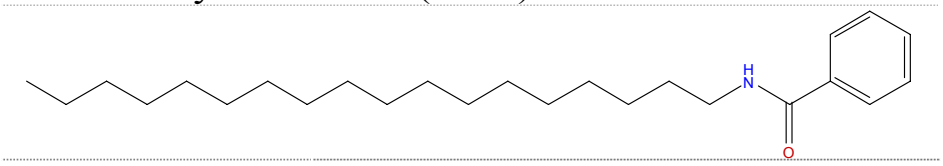

plane group $\quad p 1$

crystal class oblique

$\begin{array}{ll}Z & 1 \\ Z^{\prime} & 1\end{array}$

molecular $\mathrm{C}(\mathrm{s})$

symmetry

site symmetry 1

molecular $\quad 373.62$

weight $(\mathrm{Da})$

multicomponent no

Qian, P., Nanjo, H., Yokoyama, T. \& Suzuki, T. M.

Chem. Lett. 1999 1229-1230.

\section{Record 312}

5-formyl-3,3"'-dipentyl-2,2':5',2":5",2"':5"',2"'"-

quinquethiphene

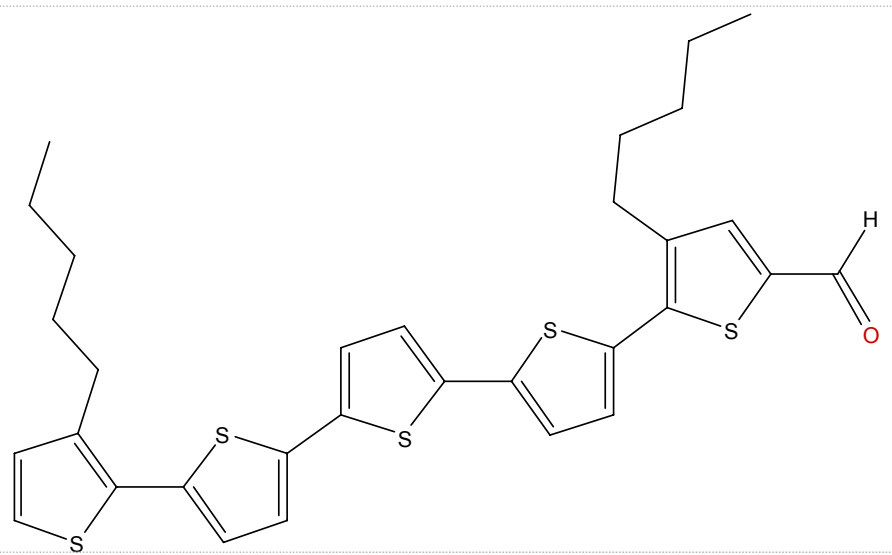

plane group $\quad p 1$

crystal class oblique

Z $\quad 1$

$Z^{\prime}-1$

molecular $\quad \mathrm{C}(\mathrm{s})$

symmetry

site symmetry 1

molecular $\quad 580.91$

weight (Da)

multicomponent no

Müller, H., Petersen, J., Strohmaier, R., Gompf, B., Eisenmenger, W., Vollmer, M. S. \& Effenberger, F. Adv. Mater. 1996 8, 733-737.

\section{Record 313}

octadecyl tetradecyl carbamate O14-N18 (3)

plane group $\quad p 1$

crystal class oblique

Z 1

$Z^{\prime} \quad 1$

molecular $\quad \mathrm{C}(\mathrm{s})$

symmetry

site symmetry 1

molecular $\quad 509.89$

weight (Da)

multicomponent no

Matzger, A. J. \& Kim, K. Polym. Mater. Sci. Eng. 2003 89, 825-826.

\section{Record 314}

5-bromo-3,3-dipentyl-2,2':5',2:5,2'":5'",2-

quinquethiophene (5a) 


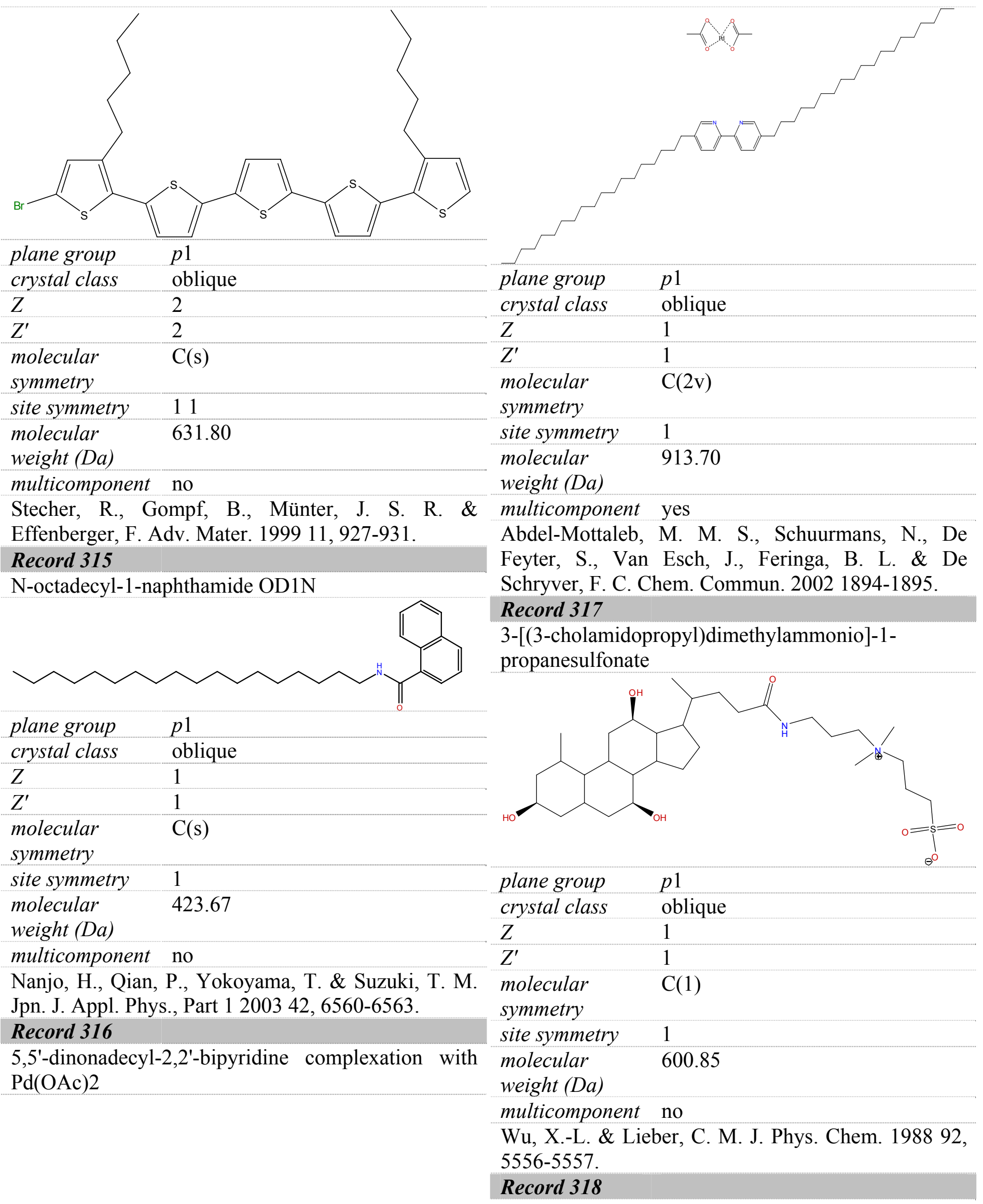


5-Hydroxymethyl-3,3-dipentyl-2,2':5',2:5,2"':5"',2quinquethiophene (3a)

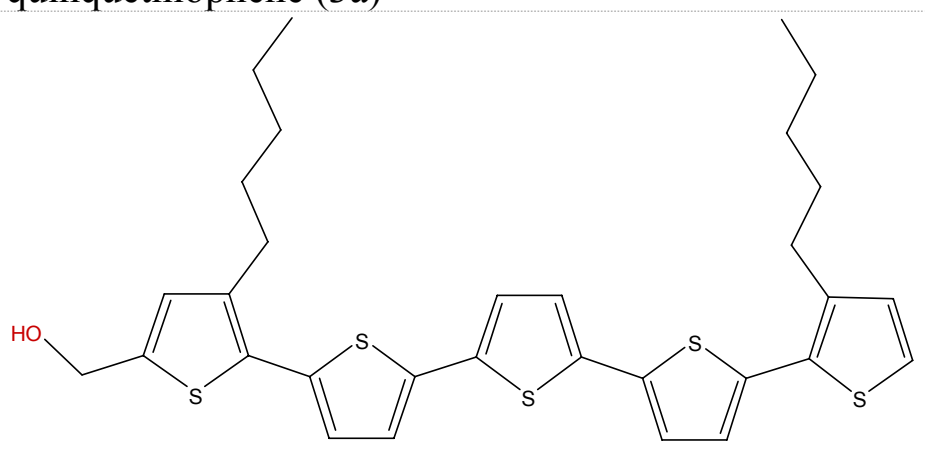

\begin{tabular}{ll} 
plane group & $p 1$ \\
crystal class & oblique \\
\hline$Z$ & 2 \\
\hline$Z^{\prime}$ & 2 \\
\hline $\begin{array}{l}\text { molecular } \\
\text { symmetry }\end{array}$ & $\mathrm{C}(\mathrm{s})$ \\
site symmetry & 11 \\
\hline $\begin{array}{l}\text { molecular } \\
\text { weight (Da) }\end{array}$ & 582.93 \\
multicomponent & no \\
\hline
\end{tabular}

Stecher, R., Gompf, B., Münter, J. S. R. \& Effenberger, F. Adv. Mater. 1999 11, 927-931.

\section{Record 319}

5,5'-dinonadecyl-2,2'-bipyridine (C19BiPyC19)

\begin{tabular}{|c|c|}
\hline plane group & $p 1$ \\
\hline crystal class & oblique \\
\hline$Z$ & 1 \\
\hline$Z^{\prime}$ & 1 \\
\hline $\begin{array}{l}\text { molecular } \\
\text { symmetry }\end{array}$ & $C(2 \mathrm{v})$ \\
\hline site symmetry & 1 \\
\hline $\begin{array}{l}\text { molecular } \\
\text { weight }(D a)\end{array}$ & 689.19 \\
\hline
\end{tabular}

Abdel-Mottaleb, M. M. S., Schuurmans, N., De Feyter, S., Van Esch, J., Feringa, B. L. \& De Schryver, F. C. Chem. Commun. 2002 1894-1895.

\section{Record 320}

octadecyl $2-(2-$ nonadecanamidopropanamido)propanoate (C18-AlaAla-OC18)

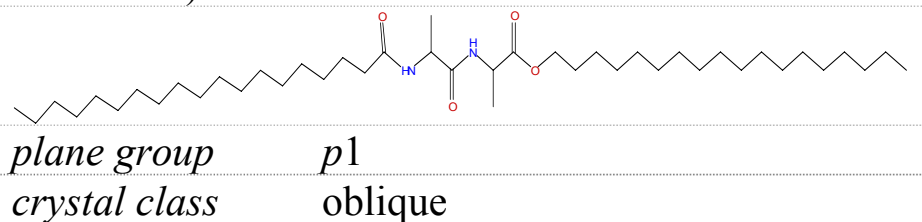
Z 1

$Z^{\prime} \quad 1$

molecular $\quad \mathrm{C}(1)$

symmetry

site symmetry 1

molecular

693.14

weight (Da)

multicomponent no

Hoeppener, S., Chi, L. F., Wonnemann, J., Erker, G. \& Fuchs, H. Surf. Sci. 2001 487, 9-14.

\section{Record 321}

4,4'-dihexyloxy-2-hydroxy-azobenzene

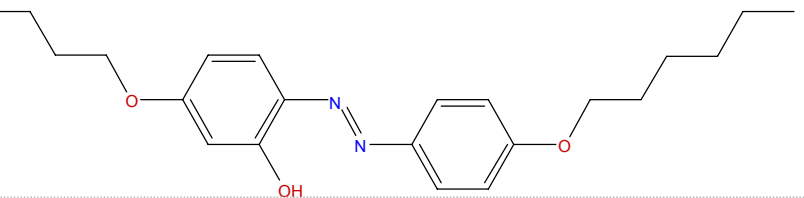

plane group $\quad p 1$

crystal class oblique

$Z \quad 1$

$Z^{\prime} \quad 1$

molecular $\quad \mathrm{C}(\mathrm{s})$

symmetry

site symmetry 1

molecular $\quad 398.54$

weight (Da)

multicomponent no

Jin, J., Li, Y., Zhang, Y., Yang, W., Chen, X., Song, Y., Zhao, Y., Bai, Y., Li, T. \& Jiang, L. Colloids Surf., A 2002 198-200, 249-253.

\section{Record 322}

17,19-dotetracontadiyne 


\begin{tabular}{l} 
plane group $\quad$ oblique \\
crystal class \\
$Z \begin{array}{l}\text { Z' } \\
\text { molecular }\end{array}$ \\
symmetry $\quad$ C(s) \\
site symmetry $\quad 1$ \\
molecular $\quad 583.07$ \\
weight (Da) \\
multicomponent no \\
Takajo, D., Fujiwara, E., Irie, S., Nemoto, T., Isoda, \\
S., Ozaki, H., Toda, N., Tomii, S., Magara, T., \\
Mazaki, Y. \& Yamamoto, G. J. Cryst. Growth 2002 \\
237-239, 2071-2075. \\
\hline
\end{tabular}

\section{Record 323}

1-triacontanol

$\begin{array}{ll}\begin{array}{l}\text { plane group } \\ \text { crystal class }\end{array} & \text { oblique } \\ Z & 2 \\ Z^{\prime} & 2\end{array}$

molecular $\mathrm{C}(\mathrm{s})$

symmetry

$\begin{array}{ll}\text { site symmetry } & 11 \\ \text { molecular } & 438.81\end{array}$

weight (Da)

multicomponent no

Venkataraman, B. \& Flynn, G. W. Atomic Force

Microscopy/Scanning Tunneling Microscopy 2,

[Proceedings of the U.S. Army Soldier Systems

Command, Natick Research, Development and Engineering Center Atomic Force Microscopy/Scanning Tunneling Microscopy (AFM/STM) Symposium], 2nd, Natick, Mass., June 7-9, 19941997 83-90.

\section{Record 324}

trans-4-hydroxy-30-trifuoromethylazobenzene<smiles>Oc1ccc(/N=N/c2cccc(C(F)(F)F)c2)cc1</smiles>

plane group $\quad p 1$

crystal class oblique

Z 2

$Z^{\prime}-2$

molecular $\quad \mathrm{C}(\mathrm{s})$

symmetry

site symmetry $\quad 11$

molecular $\quad 266.22$

weight (Da)

multicomponent no

Feng, C. L.; Zhang, Y.; Jin, J.; Song, Y.; Xie, L.; Qu, G.; Jiang, L.; Zhu, D. Surf. Sci. 2002, 513, 111-118.

\section{Record 325}

cis-4-hydroxy-30-trifuoromethylazobenzene $\mathrm{HO}$

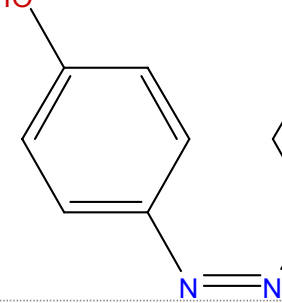

plane group $\quad p 1$

crystal class oblique

$\begin{array}{ll}Z & 2 \\ Z^{\prime} & 2\end{array}$

molecular $\mathrm{C}(\mathrm{s})$

symmetry

site symmetry 11

molecular $\quad 266.22$

weight (Da)

multicomponent no

Feng, C. L.; Zhang, Y.; Jin, J.; Song, Y.; Xie, L.; Qu, G.; Jiang, L.; Zhu, D. Surf. Sci. 2002, 513, 111-118.

\section{Record 326}

hexakis(zinc (II) porphyrinato)benzene (Zn1) and bipyridine 


\begin{tabular}{|c|c|}
\hline & site symmetry \\
\hline & $\begin{array}{l}\text { molecular } \\
\text { weight }(D a)\end{array}$ \\
\hline & $\begin{array}{l}\text { multicomponent no } \\
\text { Brandow, S. L., DiLella, D. P., Colton, R. J. \& } \\
\text { Shashidhar, R. J. Vac. Sci. Technol., B 1991 9, 1115- } \\
1118 \text {. }\end{array}$ \\
\hline & Record 328 \\
\hline & plane group \\
\hline & crystal class \\
\hline & $Z$ \\
\hline & $Z^{\prime}$ \\
\hline & $\begin{array}{l}\text { molecular } \\
\text { symmetry }\end{array}$ \\
\hline & site symmetry \\
\hline & $\begin{array}{l}\text { molecular } \\
\text { weight }(D a)\end{array}$ \\
\hline & multicomponent no \\
\hline plane group & Padowitz, D. F. \& Messmore, B. W. J. Phys. Chem. \\
\hline crystal class oblique & В $2000104,9943-9946$. \\
\hline$Z$ & Record 329 \\
\hline$Z^{\prime}$ & 2,6-bis(octyloxy)anthracene (3) \\
\hline $\begin{array}{l}\text { molecular } \\
\text { symmetry }\end{array}$ & \\
\hline site symmetry $\quad 11$ & \\
\hline molecular $\quad 8498.63$ & \\
\hline weight $(D a)$ & plane group \\
\hline multicomponent yes & crystal class \\
\hline Elemans, J. A. A. W., Lensen, M. C., Gerritsen, J. & $Z$ \\
\hline W., van Kempen, H., Speller, S., nolte, R. J. M. \& & $Z^{\prime}$ \\
\hline $\begin{array}{l}\text { Rowan, A. E. Adv. Mater. } 2003 \text { 15, 2070-2073. } \\
\text { Record } 327\end{array}$ & $\begin{array}{l}\text { molecular } \\
\text { symmetry }\end{array}$ \\
\hline 4-n-hexyl-4'-cyanobiphenyl (6CB) & site symmetry \\
\hline & $\begin{array}{l}\text { molecular } \\
\text { weight }(D a)\end{array}$ \\
\hline & multicomponent no \\
\hline $\mathrm{N} \equiv$ & $\begin{array}{l}\text { Hansen, T., Itoua, S., Kamounah, F. S., Christensen, } \\
\text { J. B., Bjørnholm, T., Schaumburg, K., Bechgaard, K. }\end{array}$ \\
\hline plane group & \& Wilkes, S. B. J. Mater. Chem. 1999 9, 1107-1113. \\
\hline crystal class & Record 330 \\
\hline$Z$ & Octadecanoic acid (S)-1-phenylethylamide \\
\hline$Z^{\prime}$ & \\
\hline $\begin{array}{l}\text { molecular } \\
\text { symmetry }\end{array}$ & \\
\hline
\end{tabular}




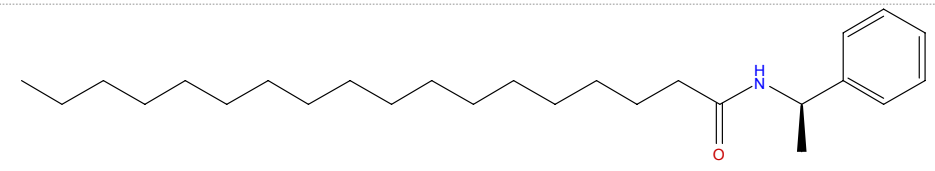

plane group $\quad p 1$

crystal class oblique

$\begin{array}{ll}Z & 1 \\ Z^{\prime} & 1\end{array}$

$Z^{\prime} \quad 1$

molecular $\quad \mathrm{C}(1)$

symmetry

site symmetry 1

molecular $\quad 387.64$

weight $(D a)$

multicomponent no

Nanjo, H., Qian, P., Yokoyama, T. \& Suzuki, T. M. Jpn. J. Appl. Phys., Part 12003 42, 6560-6563.

\section{Record 331}

didodecyldecithiophene-5-carboxylic acid

benzyl ester (10e).

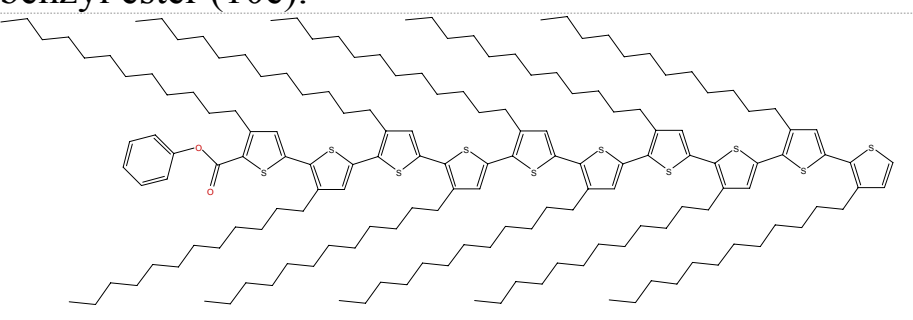

\begin{tabular}{ll} 
plane group & $p 1$ \\
crystal class & oblique \\
$Z Z$ & 1 \\
\hline$Z^{\prime}$ & 1 \\
molecular & $\mathrm{C}(\mathrm{s})$ \\
symmetry & \\
site symmetry & 1 \\
$\begin{array}{l}\text { molecular } \\
\text { weight }(\mathrm{Da})\end{array}$ & 2626.55 \\
multicomponent & no \\
\hline
\end{tabular}

Kirschbaum, T., Azumi, R., Mena-Osteritz, E. \& Bäuerle, P. New J. Chem. 1999 23, 241-250.

\section{Record 332}

N-octadecyl-2-naphthamide OD2N

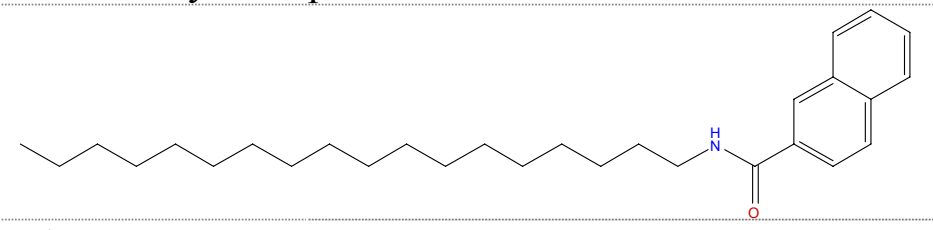

plane group $\quad p 1$

crystal class oblique
$\begin{array}{ll}Z & 1 \\ Z^{\prime} & 1 \\ \text { molecular } & \mathrm{C}(\mathrm{s}) \\ \text { symmetry } & \end{array}$

site symmetry 1

molecular $\quad 423.67$

weight (Da)

multicomponent no

Nanjo, H., Qian, P., Yokoyama, T. \& Suzuki, T. M.

Jpn. J. Appl. Phys., Part 12003 42, 6560-6563.

\section{Record 333}

dihexadecylether

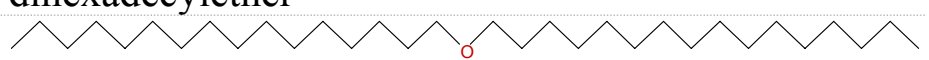

plane group $\quad p 1$

crystal class oblique

$Z^{\prime} \quad 1$

$Z^{\prime} \quad 1$

molecular $\quad \mathrm{C}(2 \mathrm{v})$

symmetry

site symmetry 1

molecular $\quad 466.87$

weight (Da)

multicomponent no

Padowitz, D. F. \& Messmore, B. W. J. Phys. Chem.

B 2000 104, 9943-9946.

\section{Record 334}

4-n-heptyloxy-4'-cyanobiphenyl (7OCB)

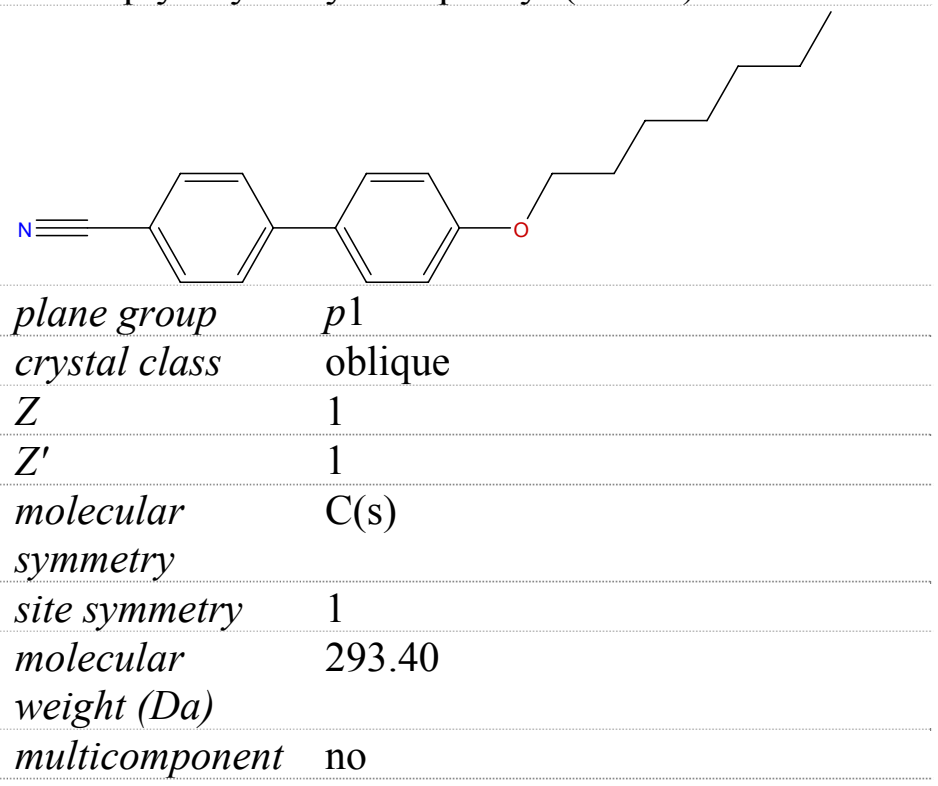

Taki, S. \& Kai, S. Jpn. J. Appl. Phys., Part 12001 40, 4187-4192.

\section{Record 335}




plane group
crystal class

Spong, J. K., Mizes, H. A., Lacomb, L. J., Dovek, M. M., Frommer, J. E. \& Foster, J. S. Nature 1989 338, 137-139.

\section{Record 336}

N-phenylstearamide

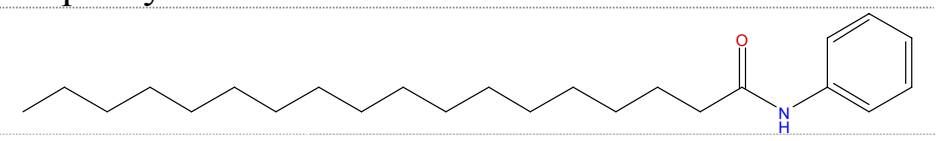

plane group $\quad p 1$

crystal class oblique

$\begin{array}{ll}Z & 1 \\ Z^{\prime} & 1\end{array}$

molecular $\mathrm{C}(\mathrm{s})$

symmetry

site symmetry 1

molecular $\quad 359.59$

weight (Da)

multicomponent no

Nanjo, H., Qian, P., Yokoyama, T. \& Suzuki, T. M. Jpn. J. Appl. Phys., Part 12003 42, 6560-6563.

\section{Record 337}

[2]catenane

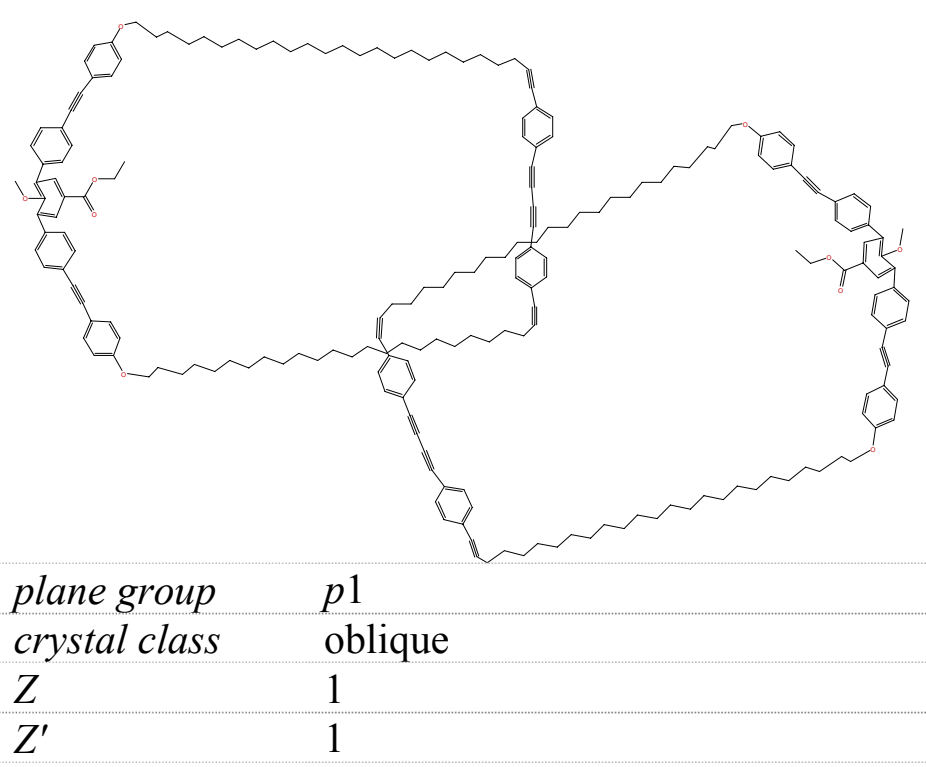

molecular $\quad \mathrm{C}(2)$

symmetry

site symmetry 1

molecular $\quad 2912.22$

weight (Da)

multicomponent no

Samorí, P., Jäckel, F., Ünsal, Ö., Godt, A. \& Rabe, J.

P. ChemPhysChem 2001 2, 461-464.

\section{Record 338}

tetratriacontane

$\begin{array}{ll}\begin{array}{l}\text { plane group } \\ \text { crystal class }\end{array} & \text { cm } \\ Z & 2 \\ Z^{\prime} & 0.5 \\ \text { molectangular } & \mathrm{C}(2 \mathrm{~h}) \\ \text { symmetry } & \end{array}$

site symmetry $\quad \mathrm{m}$

molecular $\quad 478.92$

weight (Da)

multicomponent no

Rabe, J. P. \& Buchholz, S. Makromol. Chem., Macromol. Symp. 1991 50, 261-268.

\section{Record 339}

1,3-dinonadecanoylbenzene Ketone-19

\begin{tabular}{ll} 
plane group & $\mathrm{cm}$ \\
crystal class & rectangular \\
\hline$Z$ & 6 \\
\hline$Z^{\prime}$ & 1.5
\end{tabular}




\begin{tabular}{|c|c|}
\hline $\begin{array}{l}\text { molecular } \\
\text { symmetry }\end{array}$ & $C(2 v)$ \\
\hline site symmetry & $\mathrm{mg}$ \\
\hline $\begin{array}{l}\text { molecular } \\
\text { weight }(D a)\end{array}$ & 639.09 \\
\hline multicomponent & no \\
\hline $\begin{array}{l}\text { Kim, K. \& Mat } \\
124,8772-8773 .\end{array}$ & ger, A. J. J. Am. Chem. Soc. 2002 \\
\hline Record 340 & \\
\hline heptacosane & \\
\hline plane group & $\mathrm{cm}$ \\
\hline crystal class & rectangular \\
\hline$Z$ & 2 \\
\hline$Z^{\prime}$ & 0.5 \\
\hline $\begin{array}{l}\text { molecular } \\
\text { symmetry }\end{array}$ & $C(2 v)$ \\
\hline site symmetry & $\mathrm{m}$ \\
\hline $\begin{array}{l}\text { molecular } \\
\text { weight }(D a)\end{array}$ & 380.73 \\
\hline multicomponent & no \\
\hline
\end{tabular}

Rabe, J. P., Buchholz, S. \& Askadskaya, L. Phys.

Scr. 1993 T49A, 260-263.

\section{Record 341}

Ester-19; 1,3-di-(1-heptadecyl) isophthalate

$\begin{array}{ll}\text { plane group } & \mathrm{cm} \\ \text { crystal class } & \text { rectangular } \\ Z & 2 \\ Z^{\prime} & 0.5 \\ \begin{array}{l}\text { molecular } \\ \text { symmetry }\end{array} & \mathrm{C}(2 \mathrm{v}) \\ \text { site symmetry } & \mathrm{m} \\ \text { molecular } & 643.03 \\ \text { weight (Da) } & \\ \text { multicomponent } & \text { no } \\ \text { Plass, K. E., Kim }, \mathrm{K} . \& \mathrm{Matzger}, \mathrm{A} . \mathrm{J} . \mathrm{J} . \mathrm{Am} . \mathrm{Ch}\end{array}$

Plass, K. E., Kim, K. \& Matzger, A. J. J. Am. Chem. Soc. 2004 126, 9042-9053.

\section{Record 342}

hexatriacontane

plane group $\quad c m$
crystal class $\quad \begin{aligned} & \text { rectangular } \\ & Z\end{aligned}$
$Z^{\prime}$
0.5

molecular

$\mathrm{C}(2 \mathrm{~h})$

symmetry

site symmetry $\quad \mathrm{m}$

molecular

506.97

weight $(\mathrm{Da})$

multicomponent no

Liang, W., Whangbo, M.-H., Wawkuschewski, A., Cantow, H.-J. \& Magonov, S. N. Adv. Mater. 1993 5, 817-821.

\section{Record 343}

18-hydroxy-pentatriacontane

plane group $\quad \mathrm{cm}$

crystal class rectangular

$Z \quad 2$

$Z^{\prime} \quad 0.5$

molecular $\mathrm{C}(\mathrm{s})$

symmetry

site symmetry $\quad \mathrm{m}$

molecular $\quad 508.95$

weight (Da)

multicomponent no

Claypool, C. L., Faglioni, F., Matzger, A. J., Goddard, W. A. \& Lewis, N. S. J. Phys. Chem. B 1999 103, 9690-9699.

\section{Record 344}

1-bromodocosane

$\begin{array}{ll}\text { plane group } & c m \\ \text { crystal class } & \text { oblique } \\ Z & 4 \\ Z^{\prime} & 1 \\ \text { molecular } & \mathrm{C}(\mathrm{s}) \\ \text { symmetry } & \\ \text { site symmetry } & 1 \\ \text { molecular } & 389.50 \\ \text { weight }(\mathrm{Da}) & \end{array}$

multicomponent no

Cyr, D. M., Venkataraman, B., Flynn, G. W., Black, A. \& Whitesides, G. M. J. Phys. Chem. 1996 100, 13747-13759.

\section{Record 345}

Ester-23; 1,3-di-(1-heneicosyl) isophthalate

plane group $\quad \mathrm{cm}$ 


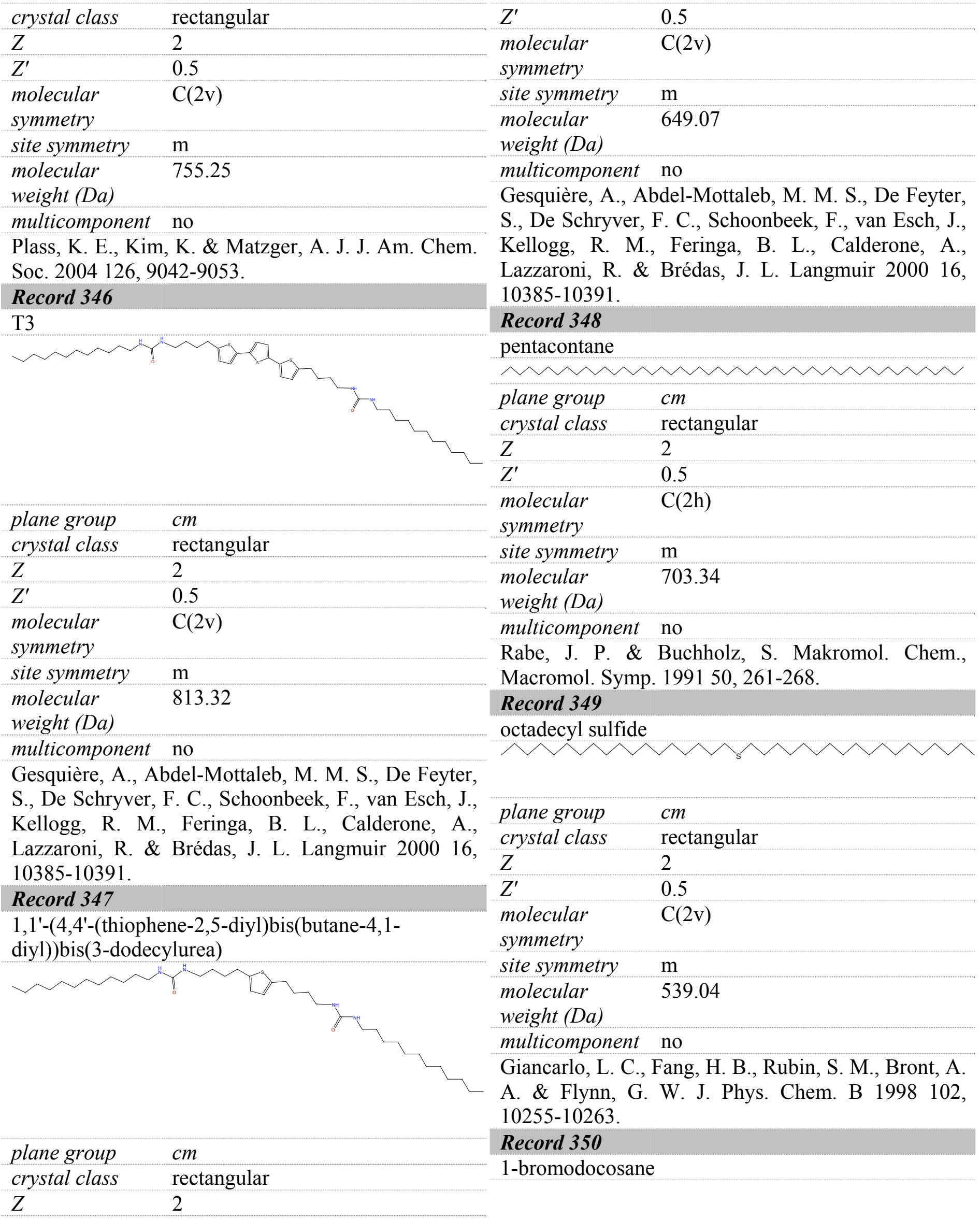




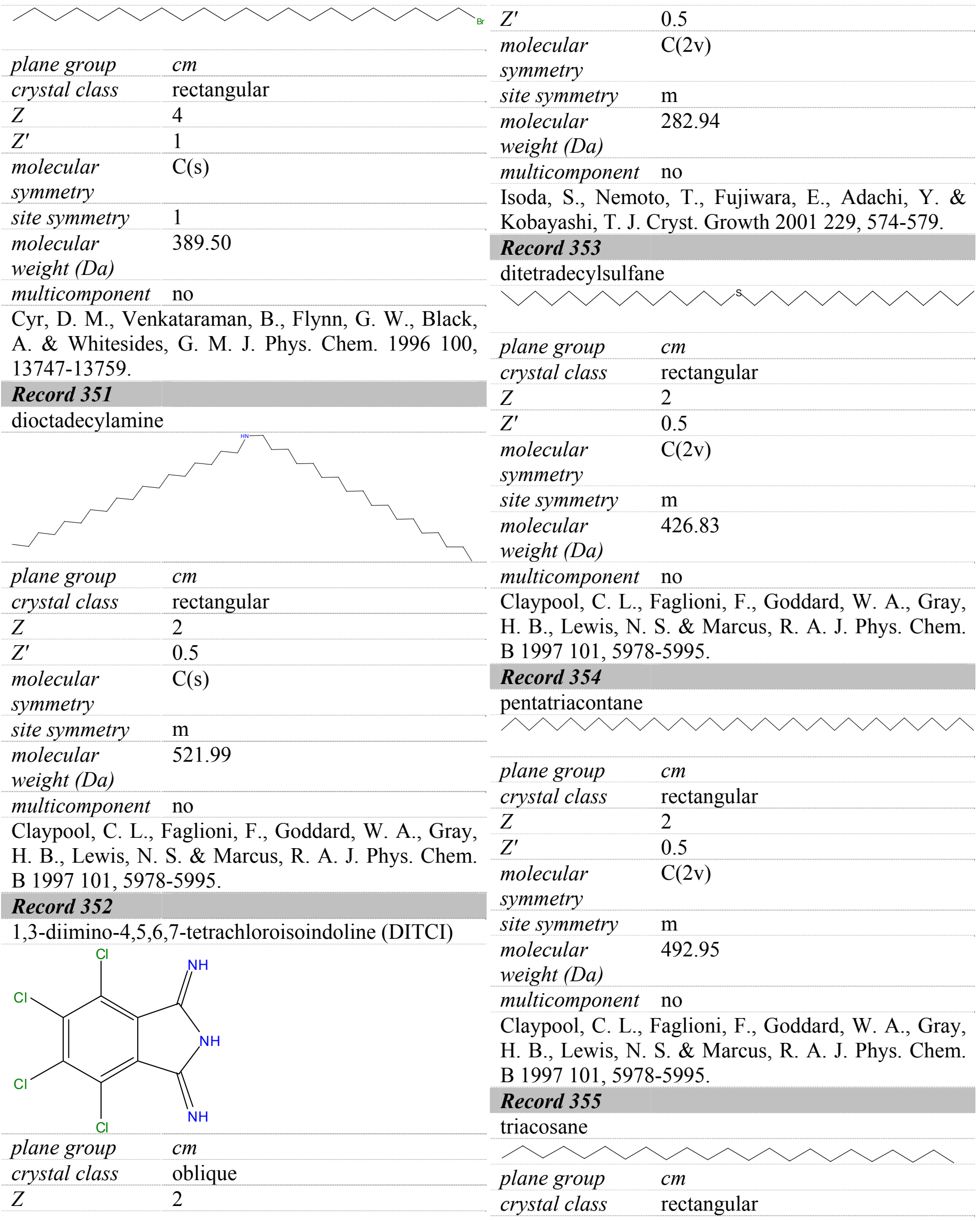


$\begin{array}{ll}Z & 2 \\ Z^{\prime} & 0.5\end{array}$

molecular $\quad \mathrm{C}(2 \mathrm{v})$

symmetry

site symmetry $\mathrm{m}$

molecular

324.63

weight (Da)

multicomponent no

Couto, M. S., Liu, X. Y., Meekes, H. \& Bennema, P. J. Appl. Phys. 1994 75, 627-628.

Record 356

hexakis-hexadecyloxytriphenylene

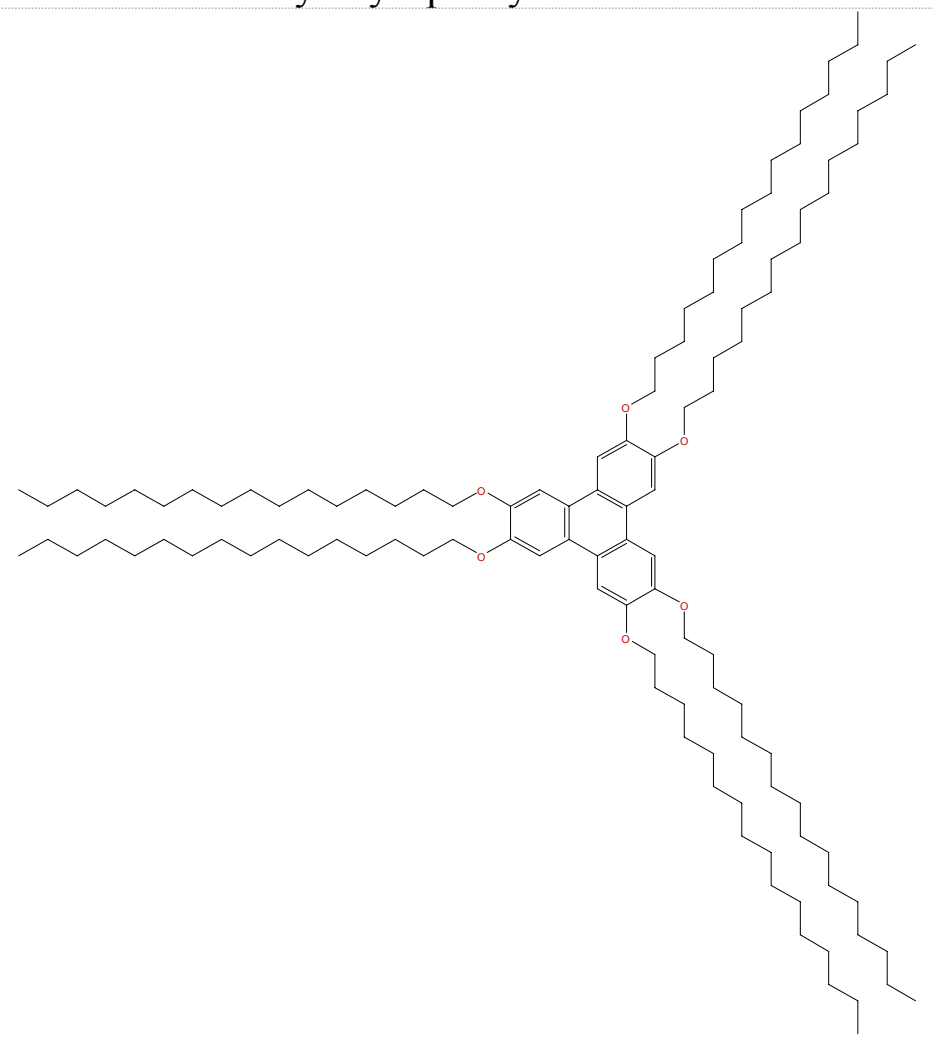

plane group $\mathrm{cm}$

crystal class rectangular

$\begin{array}{ll}Z & 2 \\ Z^{\prime} & 0.5\end{array}$

molecular $\mathrm{D}(3 \mathrm{~h})$

symmetry

site symmetry $\mathrm{m}$

molecular

1670.84

weight (Da)

multicomponent no

Rabe, J. P., Buchholz, S. \& Askadskaya, L. Synth.

Met. 1993 54, 339-349.

\section{Record 357}

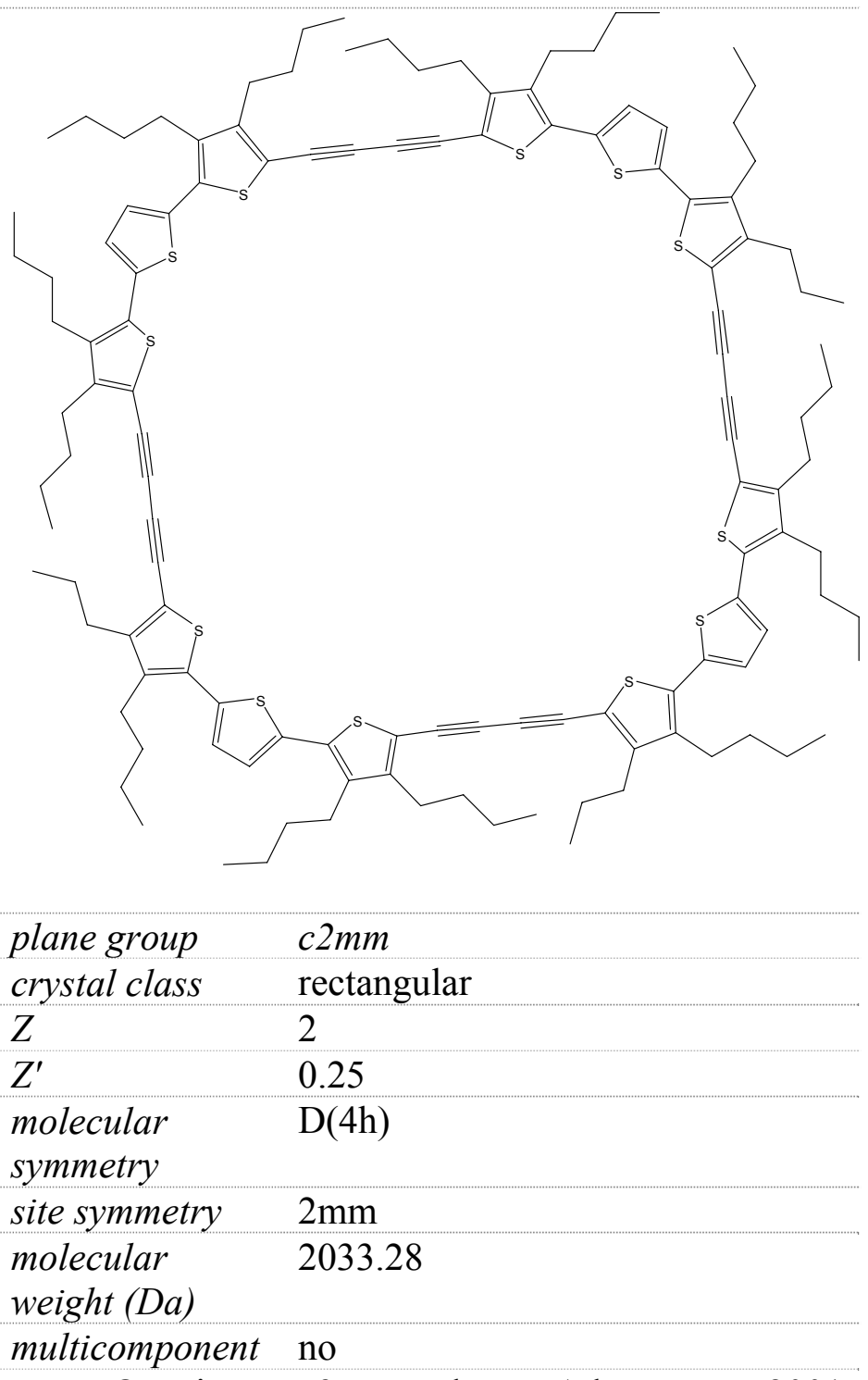

Mena-Osteritz, E. \& Bäuerle, P. Adv. Mater. 2001

13, 243-246.

\section{Record 358}

c132h34 


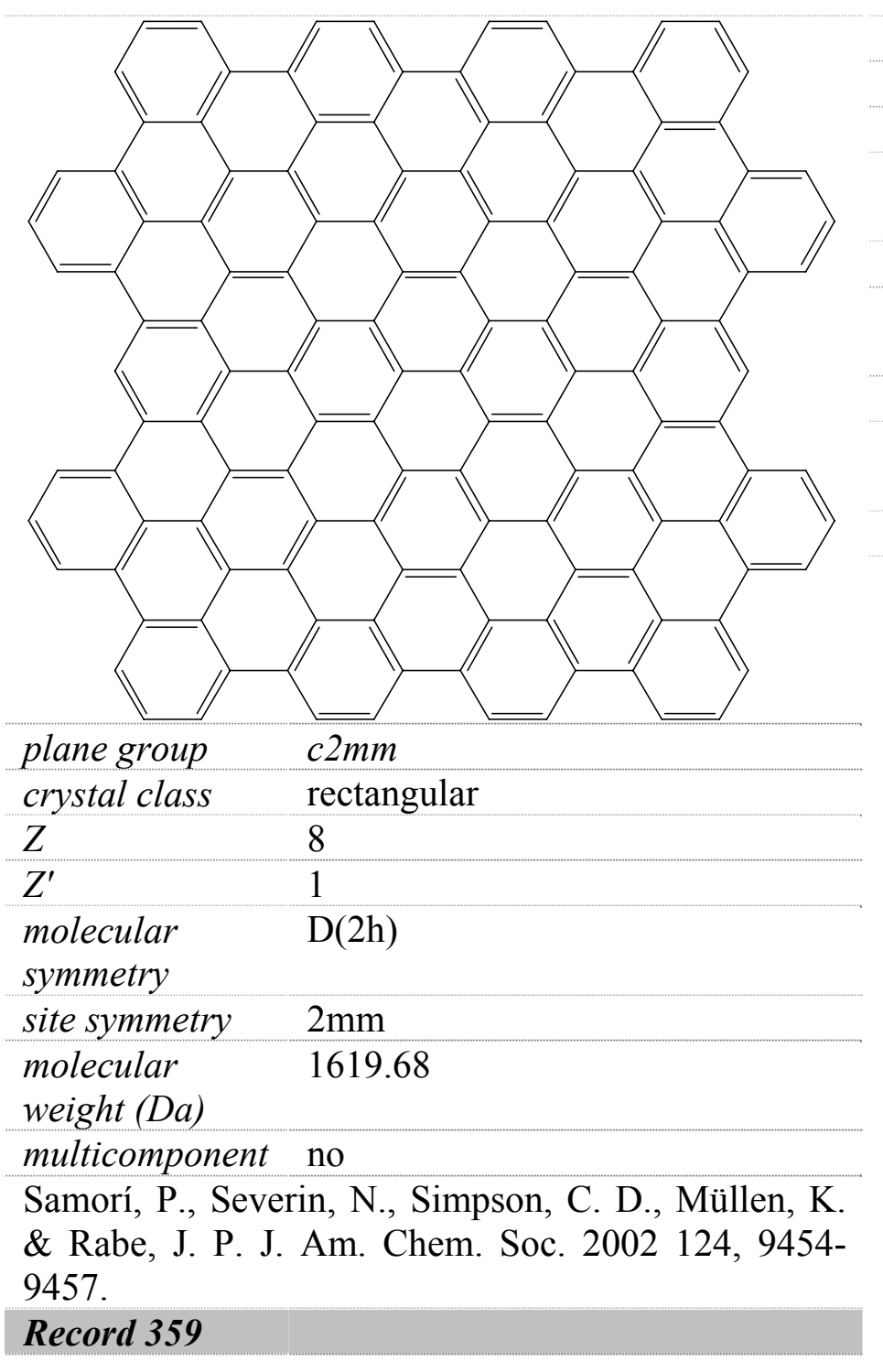

\begin{tabular}{|c|c|}
\hline crystal class & rectangular \\
\hline$Z$ & 8 \\
\hline$Z^{\prime}$ & 1 \\
\hline $\begin{array}{l}\text { molecular } \\
\text { symmetry }\end{array}$ & $\mathrm{C}(2 \mathrm{v})$ \\
\hline site symmetry & $\mathrm{m}$ \\
\hline $\begin{array}{l}\text { molecular } \\
\text { weight }(D a)\end{array}$ & 618.69 \\
\hline multicomponent & no \\
\hline $\begin{array}{l}\text { Ziener, U., Lehr } \\
\text { Chem.-Eur. J. } 20\end{array}$ & $\begin{array}{l}\text { J.-M., Mourran, A. \& Möller, M. } \\
2 \text { 8, 951-957. }\end{array}$ \\
\hline
\end{tabular}<smiles>c1ccc(-c2nc(-c3cc(-c4ccncc4)cc(-c4ccncc4)n3)cc(-c3cc(-c4ccncc4)cc(-c4ccncc4)n3)n2)cc1</smiles> 